\title{
FAST AND ROBUST \\ MULTI-DIMENSIONAL CARDIAC MAGNETIC RESONANCE IMAGING
}

\author{
DISSERTATION \\ for the award of the degree \\ "Doctor rerum naturalium (Dr. rer. nat.)" \\ of the Georg-August-University Göttingen \\ within the doctoral program \\ Physics of Biological and Complex Systems \\ of the Georg-August-University School of Science (GAUSS) \\ submitted by \\ SEBASTIAN ROSENZWEIG \\ from Würzburg
}

Göttingen, 2020 
THESIS COMMITTEE

FIRST REFEREE AND SUPERVISOR

Prof. Dr. Martin Uecker

Institute for Diagnostic and Interventional Radiology

University Medical Center Göttingen

SECOND REFEREE

Prof. Dr. Ulrich Parlitz

Biomedical Physics Group

Max-Planck-Institute for Dynamics and Self-Organization Göttingen

Prof. Dr. Gerlind Plonka-Hoch

Institute for Numerical and Applied Mathematics

Georg-August-University Göttingen

EXTERNAL REFEREES

THIRD REFEREE

Prof. Dr. Rudolf Stollberger

Institute of Medical Engineering

Graz University of Technology

MEMBERS OF THE EXAMINATION BOARD

Prof. Dr. Tim Salditt

Institute for X-Ray Physics

Georg-August-University Göttingen

Prof. Dr. Susann Boretius

Functional Imaging Laboratory

German Primate Center

PD Dr. Peter Dechent

Cognitive Neurology

University Medical Center Göttingen

Date of oral examination: June 10, 2020 


\section{ACKNOWLEDGMENTS}

Zuallererst möchte ich meinem Betreuer Prof. Dr. Martin Uecker danken, durch den ich die Möglichkeit hatte das faszinierende Feld der Magnetresonanztomographie zu entdecken. Seine Fähigkeit komplizierte Sachverhalte in kürzester Zeit zu durchschauen und auf bekannte Grundlagen zurückzuführen haben viel zu meinem eigenen Verständnis der MRT beigetragen. Seine Kreativität, seine Offenheit für Fragen und Diskussion, sowie seine stetige Unterstützung haben diese Arbeit erst möglich gemacht.

Ein weiterer Dank geht an Prof. Dr. Ulrich Parlitz und Prof. Dr. Gerlind PlonkaHoch, die als Teil meines Thesis Advisory Committees durch bereichernde und motivierende Diskussionen wertvolle Hinweise zu meiner Arbeit beisteuerten. Dem Max-Planck Institut, allen genannten Prüfern sowie Prof. Dr. Tim Salditt und Dr. Volkert Roeloffs möchte ich danken, dass sie mir eine Präsenz-Verteidigung ermöglicht haben - obgleich aufgrund der Corona-Pandemie zwischenzeitlich auch eine Online-Verteidigung im Gespräch war.

Dafür, dass sie bei meiner Verteidigung dabei waren und ich diese im Anschluss nicht ganz alleine feiern musste geht ein großer Dank an Felix, Anneke, Gerda, Chrissi, Jakob, Jasper und Nick.

Sodann möchte ich mich bei meinen alten und neuen Kollegen bedanken. Ihr hattet einen großen Anteil daran, dass ich mich wirklich zu jeder Zeit sehr wohl gefühlt habe in dieser Arbeitsgruppe. Neben der fachlichen Unterstützung und der stets positiven Arbeitsatmosphäre waren für mich vor allem auch unsere gemeinsamen Kaffeepausen - die während des Lockdowns sogar online stattfanden - sehr bereichernd. Es ist wahrlich nicht selbstverständlich in einer Arbeitsgruppe arbeiten zu dürfen, in der man sich so gut versteht und sich selbst außerhalb der Arbeitszeit zu Film-, Spiel- oder Kneipenabenden trifft!

Besonders danken möchte ich dabei meinem Bürokollegen Christian. Ich konnte wirklich viel von dir lernen und dein fachliches und technisches Verständnis hat mir einiges an Arbeit und Zeit erspart.

Auch an Nick geht ein großer Dank! Unsere zahlreichen Gespräche fachlicher aber gerade auch privater Natur waren und sind für mich persönlich von großem Wert. Außerdem ist dein Anteil an der allgemeinen Arbeitsatmosphäre in dieser 
Gruppe nicht hoch genug einzuschätzen!

Ein Dank geht auch an meine weiteren Co-Autoren - so ich sie bisher noch nicht erwähnt habe -, Prof. Dr. Jens Frahm, Dirk und Robin. Sowohl in wissenschaftlicher als auch in persönlicher Hinsicht habe ich die Zusammenarbeit mit euch sehr geschätzt.

Außerdem bin ich all jenen Freunden dankbar, die sich auf die Suche nach Rechtschreibfehlern und Ungereimtheiten in meiner Arbeit gemacht, oder mir wertvolles Feedback zu meinem Vortrag - trotz manchmal schlechter Internetverbindung - gegeben haben: Jakob, Jasper und Martina, Nico, Andi und Jan, Jakob und Jost, Xiaoqing und Zhengguo und natürlich die ganze Arbeitsgruppe!

Ich möchte mich bei Prof. Dr. Mehdi Moghari für die Möglichkeit bedanken ein halbes Jahr in Boston forschen zu dürfen, und bei Aashay, Lukas und Nils, die mir in dieser Zeit persönlich eine große Hilfe waren! Ich bin wirklich froh mit euch das Büro geteilt haben zu dürfen.

Ben \& Jen, Owen, Andrea, Kevin, AJ, Celina, Timothy and Laura, I am deeply thankful for your community and support during my time in Boston and beyond. You have been such a great blessing to me and I thank God for the gift of your friendship!

Felix, Jule, Leonie, Günni, Lea, Fabio, Micha, Christian B. und Christian K., euch möchte ich ebenfalls noch einen großen Dank aussprechen. Die Gespräche mit euch und unsere Gemeinschaft im Glauben sind mir wahrlich viel wert und waren mir eine große Stütze!

Danke Judith, für unsere gemeinsame Zeit - du hast mein Leben so sehr bereichert!

Mein Studium und diese Arbeit wären für mich ohne die uneingeschränkte Unterstützung, Geduld und Hilfe meiner Eltern und meiner Schwester schwer vorstellbar. Ich konnte mich immer auf euch verlassen und ihr wart immer da - gerade in den persönlich schweren Zeiten. Vielen Dank!

"[...] Wer von der Erde ist, der ist von der Erde und redet von der Erde. Der vom Himmel kommt, ist über allen." Joh 3:31 


\section{CONTENTS}

I INTRODUCTION

2 MRI THEORY $\quad 7$

2.1 Fundamental Principles of MRI . . . . . . . . . . . . . . 7

2.1.1 The Nuclear Spin . . . . . . . . . . . . . . . . 7

2.1.2 The Nuclear Zeeman Effect . . . . . . . . . . . . . 8

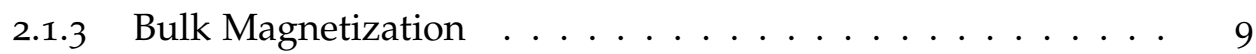

2.1.4 Bloch Equation .................... 10

2.1.5 Conclusion .................. 11

2.2 The Pulsed NMR Experiment . . . . . . . . . . . . . . 11

2.2.1 The MRI System .................. . . II

2.2.2 Slice-Selective Excitation . . . . . . . . . . . . 12

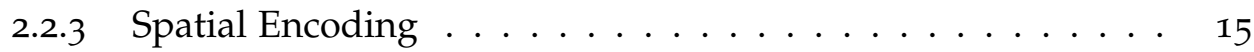

2.2.4 k-space Trajectory . . . . . . . . . . . . . 17

2.2.5 Gradient-Echo Sequences .............. . 19

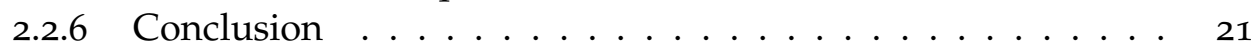

2.3 Concepts of Modern Image Reconstruction and Data Correction . . 21

2.3.1 Parallel Imaging and Compressed Sensing . . . . . . . . . . 21

2.3.2 Non-Cartesian Image Reconstruction . . . . . . . . . . . 27

2.3.3 Gradient Delay Correction ............. 31

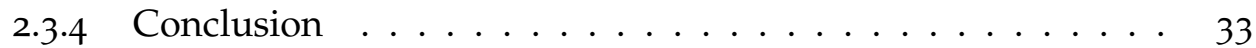

2.4 Conventional Methods in Cardiac MRI . . . . . . . . . . . . 35

2.4.1 Cardiac Gating using the Electrocardiogram . . . . . . . . . 35

2.4.2 Respiratory Monitoring and Breath-hold Commands . . . . . 37

2.4.3 Whole Heart Coverage . . . . . . . . . . . . . 38

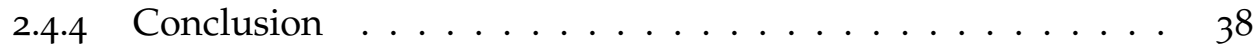

2.5 Advanced Methods for Multi-Dimensional Cardiac MRI . . . . . . . 39

2.5.1 Approaches Towards the Third Spatial Dimension . . . . . 39

2.5.2 Exploiting Temporal Correlations . . . . . . . . . . 47

2.5.3 Self-Gating and the Concept of Time-Delayed Embedding . . 53

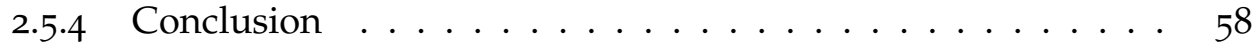

3 SIMPLE AUTO-CALIBRATED GRADIENT DELAY ESTIMATION FROM FEW SPOKES USING RADIAL INTERSECTIONS (RING) 59

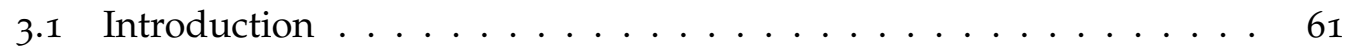

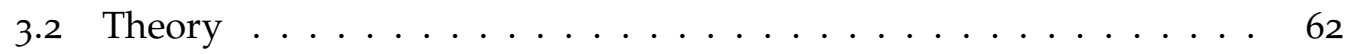

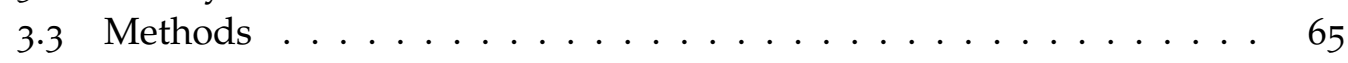


3.4 Results ........................ 66

3.5 Discussion . . . . . . . . . . . . . . . . . 69

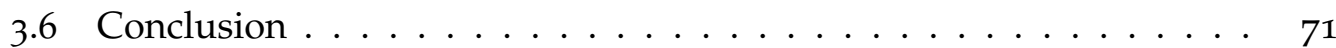

3.7 Acknowledgements . . . . . . . . . . . . . $7^{71}$

3.8 Appendix: Derivation of the Gradient Delay Ellipse Model . . . . . . 71

3.9 Supplementary Material . . . . . . . . . . . . . . . . . 73

3.9.1 Accuracy of the Intersection Point Determination . . . . . . 73

3.9 .2 NUFFT Reconstructions . . . . . . . . . . . . 76

4 RING FOR INTERACTIVE REAL-TIME MRI 79

4.1 Methods ........................... 79

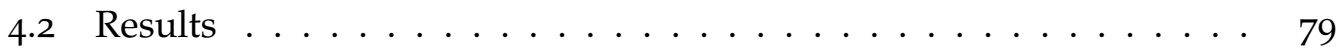

4.3 Discussion ..................... 81

4.4 Conclusion ........................ 81

5 Simultaneous MUlti-SLiCE MRI USing CARTESian AND RAdial

FLASH AND REGULARIZED NONLINEAR INVERSION: SMS-NLINV 83

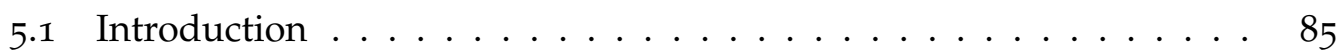

5.2 Theory ........................... 85

5.2.1 SMS Encoding and Excitation Pulses . . . . . . . . . 85

5.2 .2 Image Reconstruction . . . . . . . . . . . . . . . 86

5.2.3 Implementation for Cartesian and non-Cartesian Data . . . . 88

5.2 .4 Sampling Schemes ... . . . . . . . . . . . . . . . . 90

5.2 .5 Post-Processing . . . . . . . . . . . . . . . . . 92

5.3 Methods . . . . . . . . . . . . . . . . . 92

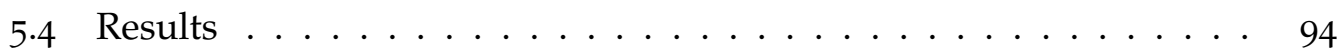

5.4.1 Cartesian Data . . . . . . . . . . . . . . . . . . . 94

5.4 .2 Radial Data . . . . . . . . . . . . . . . . . . . . . . 96

5.5 Discussion . . . . . . . . . . . . . . . . . . . 99

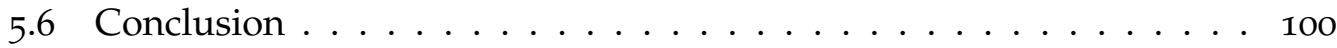

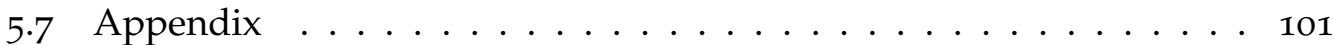

5.7 .1 Glossary . . . . . . . . . . . . . . . . . 101

5.7.2 Derivative and Adjoint of the Forward Operator . . . . . 101

$5 \cdot 7 \cdot 3$ Weighting Matrix . . . . . . . . . . . . . 102

5.8 Supplementary Material . . . . . . . . . . . . . . . 102

$\begin{array}{llr}6 & \text { REAL-TIME IMAGING USING SMS-NLINV } & 107\end{array}$

6.1 Theory . . . . . . . . . . . . . . . . . 107

6.2 Methods ...................... 110

6.3 Results ....................... 110

6.4 Discussion . . . . . . . . . . . . . . . . . 112

6.5 Conclusion . . . . . . . . . . . . . . . 112 
7 CARDIAC AND RESPIRATORY SELF-GATING IN RADIAL MRI USING AN ADAPTED SINGULAR SPECTRUM ANALYSIS (SSA-FARY) 113

7.1 Introduction . . . . . . . . . . . . 115

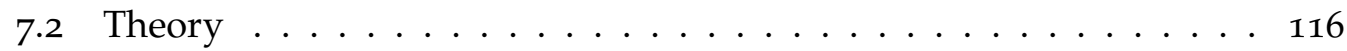

7.2.1 Correction of the AC Data . . . . . . . . . . 117

7.2.2 Dimensionality Reduction Methods ........... 118

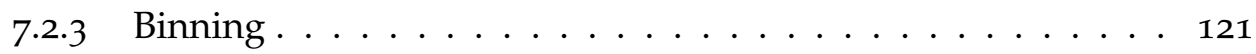

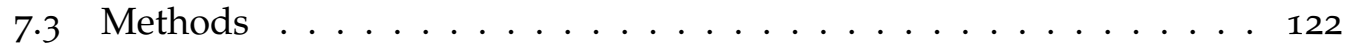

7.3.1 Numerical Simulations .............. 122

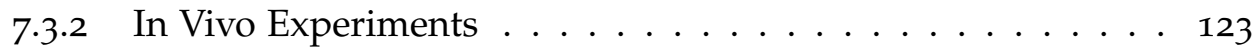

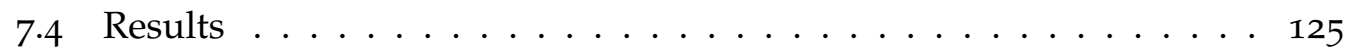

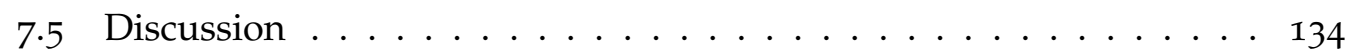

7.6 Conclusion . . . . . . . . . . . . . . . . . . . . . . . . . . . . . . . . . . . .

7.7 Acknowledgements . . . . . . . . . . . . . . . . . . . . . . . . . . . . . . . . . . . . . . . .

7.8 Appendix: Details for Numerical Simulations . . . . . . . . . . 138

7.9 Supplementary Material . . . . . . . . . . . . . 138

7.9.1 Numerical Simulations with Varying Simulation Parameters 139

7.9.2 SSA-FARY Gated bSSFP Reconstruction using Conventional

Gridding . . . . . . . . . . . . . . . . . . . . . . 139

7.9.3 SSA-FARY Gated Reconstructions for a Single-Slice RF-Spoiled Gradient-Echo Sequence . . . . . . . . . . . . . . . 143

7.9.4 Respiratory Signal Comparison for Irregular Breathing . . . . 146

7.9.5 SSA-FARY Gated Reconstructions for Highly Irregular Breathing, Yawning and a Pronounced Heart Rate Variability. . . . . 146

7.9.6 Movies of the In Vivo Experiments . . . . . . . . . . . . 148

8 SUMMARY, OUTLOOK AND CONCLUSION

A GRAdient delay estimation Using the (AC-)AdAptive method 157

$\begin{array}{lr}\text { ACRONYMS } & 159\end{array}$

$\begin{array}{lr}\text { BIBLIOGRAPHY } & 161\end{array}$

$\begin{array}{lr}\text { CURRICULUM VITAE } & 189\end{array}$ 



\section{INTRODUCTION}

In 1973 Paul Lauterbur published an essay entitled Image Formation by Induced Local Interactions: Examples Employing Nuclear Magnetic Resonance [1], which did not only earn him the shared Nobel Prize in Medicine or Physiology 2003 but also laid the foundation for one of today's most powerful and valuable imaging modalities in hospitals and clinics worldwide: Magnetic Resonance Imaging (MRI). In the only one page long manuscript Lauterbur introduced the concept of image formation using magnetic field gradients. Sir Peter Mansfield, who shared the Nobel prize with Lauterbur, further developed and formalized the mathematical concept of spatial encoding using gradient fields, paving the way for MRI to become a clinically useful modality [2-4]. While the first human thorax Magnetic Resonance (MR) image was published by Damadian et al. in 1977 [5], the Aberdeen group around John Mallard was the first to built a practical human MRI scanner in the early 1980's [2]. The first step towards cardiac MRI was done in 1981, when Hawkes et al. obtained an MR image of a human heart [6]. However, commercially available MRI systems were not provided by vendors until 1985, which marks the start of the triumph of clinically applied MRI.

MRI's great popularity and value stems from being a non-invasive imaging technique, which can generate not only structural anatomic images but also offers a variety of additional insights, such as flow or diffusion information in multiple dimensions. Compared to techniques like Computed Tomography (CT), MRI possesses a superior and furthermore adjustable soft-tissue contrast. For its versatile applications MRI has become an almost indispensable tool in today's clinical practice. While brain and spine scans make up more than half of all clinically performed MRI studies, cardiac MRI examinations account for only one percent [7]. This figure, however, should not hide the key role that this modality plays in the detection and characterization of myocardial diseases through its diagnostic and prognostic power [8]. Among others, the field of application of cardiac MRI spans the assessment of myocardial ischemia and tissue characterization, coronary artery, vascular and inflammatory heart disease, as well as hypertrophic cardiomyopathy [9]. Furthermore, it is the gold standard method for cardiac structure and function assessment [10].

Apart from the great value that this modality adds to our healthcare, MRI in general - and cardiac MRI in particular - has faced one major limitation from the very beginning of its conception: it is slow! Consequently, the quest for speed has motivated engineers and scientists ever since. Besides significant improvements in hard- 
ware components, such as superconducting magnets, magnetic field gradient coils and radio-frequency (RF) coils, new pulse sequences and novel image reconstruction techniques have been conceived to accelerate MRI scans [11]. One early milestone was set in 1986 by Haase et al. by introducing the Fast Low-Angle Shot (FLASH) sequence [12]. Their proposed combination of RF pulses and magnetic field gradients allowed for the acquisition of entire images in just a few seconds, while being robust towards static magnetic field homogeneities and chemical shift artifacts. The FLASH sequence became one of the most widely used pulse sequences and was implemented by all major vendors. Another substantial game changer was the invention of parallel imaging, i.e. the use of multiple receive coils for signal acquisition as pioneered by Sodickson et al. in 1997, which can accelerate the acquisition time by a factor of two or more [13]. The latest landmark for rapid MRI was set by Lustig et al. [14] and Block et al. [15] with the introduction of compressed sensing and iterative image reconstruction to the field of MRI. Compressed sensing enables image reconstruction from signals acquired at sub-Nyquist sampling rates, thus cutting down the required scan time even further.

Although cardiac MRI could clearly profit from those general technical advances, it has always occupied a special role amidst MRI applications for the great challenges posed by cardiac and respiratory motion. In the early years of MRI, data acquisition was not fast enough to temporally resolve the cardiac cycle. Therefore, a clever idea borrowed from CT [16] was adopted, which even today remains the clinical standard: the use of the electrocardiogram (ECG) for gated scanning $[17,18]$. By constantly monitoring the electrical excitation of the heart, individual heart phases can be detected and the data acquisition corresponding to each heart phase can be distributed over multiple heartbeats. This allows for the generation of a single, spatially and temporally well resolved synthetic heartbeat from various actual ones. This technique is known by the term CINE MRI. Nevertheless, in the decade after the first MR image of a human heart, only minor improvements in the quality of cardiac MRI exams could be achieved due to the degradation by respiratory motion [11]. Consequently, cardiac MRI could not keep pace with the advances in image quality and clinical applicability that MRI soon brought to other regions of the body.

The most intuitive way to eliminate respiratory motion artifacts is the demand for breath-holds. This, however, was only feasible for standard hardware when segmented data acquisition was introduced in 1991 [19], which cut down the scan time for one CINE heartbeat - and likewise the required breath-hold time - by a factor of 8 to about 16 seconds. Other techniques avoid the need for breathholds by tracking the respiratory motion using external devices [20] or specialized sequences with navigator echoes [21].

Even today, about 30 years after its original conception, ECG gating in combination with breath-holds or external respiratory monitoring is the clinical standard 
in cardiac MRI at mosts facilities. This being said, the approach comes with various limitations that have been apparent early on. Patient preparation and the proper positioning of ECG leads is essential to obtain a suitable ECG signal, but can be cumbersome and laborious. Moreover, RF pulses, fast switching gradients and the magneto-hydrodynamic effect lead to signal distortions, which can cause mis-triggering, particularly at higher magnetic field strengths [22-25]. In extreme cases, ECG leads may even cause burn injuries [26]. Moreover, for sick patients and children repeated breath-holds can be exhausting and in certain cases might even be infeasible, particularly for babies or non-compliant individuals. The placement of respiratory belts or pneumatic pads costs time, can be error-prone and is not always applicable [27]. Finally, the interleaved acquisition of additional navigator echoes can destroy the steady-state condition in respective sequences and prolongs the measurement time. These drawbacks, the perceived potential and the impact that cardiac MRI - even in its early stages - had manifested in hospitals worldwide, have spurred further technical developments [28].

An alternative to CINE MRI is real-time imaging, which completely avoids the dependency on patient compliance and external devices. In real-time imaging, specialized acquisition and reconstruction techniques are utilized to resolve the full cardiac dynamics of each heartbeat, rendering respiratory and cardiac gating obsolete. Some techniques even allow for on-the-fly reconstructions, which - without significant time-lag - make the images directly available on the scanner [29-31]. Despite its relatively recent success, the idea of real-time imaging is not new but goes back to the very beginning of MRI, when Ordidge et al. used Echo-Planar Imaging (EPI) to generate a dynamic MRI movie of a rabbit heart [32]. Nevertheless, due to its low spatio-temporal resolution and its high demands on the hardware it was not until the turn of the century when real-time imaging started to play a more and more important role in MRI research. With novel reconstruction approaches exploiting temporal correlations, the steadily increasing computational power and the latest generation of hardware, a variety of different real-time MRI techniques have been developed over the past two decades [33-38]. Still, most of those approaches are computationally expensive and therefore do not allow for an on-the-fly reconstruction. This is not only desirable in a clinical setting but also a requirement e.g. for interventional cardiac MRI, which came up in the early 2000's [39]. In this respect, the Regularized Nonlinear Inversion (NLINV) approach proved great potential soon after its conception [40-46]. NLINV does not only yield suitable results even from highly undersampled data but also dispenses with the need for auto-calibration (AC) scans by intrinsically generating accurate coil sensitivity maps - a crucial requirement for parallel imaging. However, the fact that real-time NLINV is limited to the imaging of a single-slice at a time hinders its broader use in clinical applications. 
A peculiarity of real-time NLINV is the employed radial sampling, i.e. the acquisition of spatial frequencies on a non-Cartesian grid. In contrast, Cartesian imaging always has been and still is the most commonly used acquisition strategy in clinically applied MRI, mainly due to its robustness towards system imperfections and straight-forward image reconstruction. However, non-Cartesian imaging has proved to be particularly beneficial in terms of the temporal resolution [32]. In the beginning of the 1990's more and more researchers started to appreciate the benefits of radial imaging, although already Lauterbur utilized this sampling strategy for the very first MR image [1]. Radial acquisitions provide a number of intrinsic properties beneficial for the demands of fast MRI [47]. They are not only relatively robust towards motion artifacts but also render the spatial resolution largely independent from the number of views, which - besides the less disturbing undersampling artifacts - allows for higher acceleration factors [11, 48]. However, radially sampled data demand a more advanced reconstruction [49] and the acquisitions are significantly more prone to system imperfections, such as gradient delays, which need to be corrected for to avoid the related streaking artifacts [50].

Still, radial acquisitions are ideally suited for another strategy that has been developed to tackle the problem of motion in cardiac MRI: Self-gating. Self-gated MRI determines the cardiac and respiratory motion directly from the acquired data itself and therefore can be considered an alternative to classical CINE MRI, refraining from the use of any external devices or breath-holds. The idea of self-gating underlies the notion that cardiac and respiratory motion constitute major contributions to the acquired signal, which makes it possible to extract those motion signals by frequent sampling of the energetically dominant low spatial frequencies. This is naturally the case for radial imaging. The idea of self-gating is already quite old [51] and has been improved and refined over the past thirty years [52-57]. Still, it has never proved to be robust enough to prevail in clinical practice.

OUTLINE OF THIS THESIS Following an introduction to the basics of MR signal generation and image reconstruction in Chapter 2, we will tackle several of the previously mentioned limitations of current methods used in cardiac MRI. In particular, we will take advantage of (multi-)spatial and (multi-)temporal correlations for the goal of fast and robust multi-dimensional MRI. To this end, a reliable correction of system imperfections is mandatory, which will be the focus of Chapter 3, where we will introduce Radial Spoke Intersections for Gradient Delay Estimation (RING), a simple, fast and powerful method for the auto-calibrated estimation of gradient delays in radial imaging, which enables the elimination of streaking artifacts caused by system imperfections $[58,59]$. We will demonstrate the functionality of RING on numerical simulations, phantom and in vivo experiments. As gradient delays depend on the orientation and location of the imaging plane, gradient delay estimates must be updated if the slice position changes. Since RING requires only a 
very small number of radial spokes, it allows for a frame-by-frame gradient delay estimation and thus constitutes a valuable asset e.g. for interactive real-time MRI experiments, as we will show in Chapter 4.

In Chapter 5, we will extend the NLINV algorithm for the joint reconstruction of multiple slices using the method of Simultaneous Multi-Slice (SMS) [4042, 6o-63]. Like this, enhanced spatial frequency information can be used in addition to in-plane correlations to improve the image quality of each slice. This combination of techniques, dubbed SMS-NLINV, allows for the reconstruction of highly undersampled data, as we will demonstrate on phantom and in vivo measurements. Moreover, SMS-NLINV intrinsically guarantees time-consistency for all slices, which is favorable for the study of dynamic processes. In particular, the additional integration of temporal regularization shown in Chapter 6 allows for realtime cardiac SMS imaging. Furthermore, SMS-NLINV does not only yield image information but also provides excellent auto-calibrated coil sensitivity maps, which will be employed for parallel imaging and compressed sensing reconstructions in Chapter 7 .

The main topics of Chapter 7 will be the development of a novel dimensionalityreduction and self-gating approach called Singular Spectrum Analysis for Advanced Reduction of Dimensionality (SSA-FARY) [64, 65], which renders breath-holds and additional hardware for the detection of cardiac and respiratory motion signals in gated cardiac MRI obsolete. SSA-FARY borrows ideas from Singular Spectrum Analysis (SSA) and can be considered a Principle Component Analysis (PCA) on a time-delayed embedding. It intrinsically features data-denoising and band-pass filtering, which makes it a powerful tool to robustly detect and separate cardiac and respiratory motion signals. Additionally, it provides a natural solution to bin the data into the respective cardiac and respiratory states. In combination with an advanced image reconstruction pipeline based on parallel imaging and compressed sensing, we will demonstrate the feasibility of fast and robust multi-dimensional MRI with high spatial and temporal resolution for single-slice, SMS and volumetric Stack-of-Stars (SOS) experiments.

Finally, Chapter 8 will summarize the findings and provide an outlook towards future work. 



\section{MRI THEORY}

\subsection{FUNDAMENTAL PRINCIPLES OF MRI}

In this section we will give a brief introduction on the fundamental principles of MRI. An in-depth study of the underlying physics and all relevant mechanisms is beyond the scope of this thesis and has been described extensively in the literature. Therefore, the interested reader is referred to the introductory textbooks [66-71], which also serve as general references for this section. The structure of this section is inspired by [63].

\subsubsection{The Nuclear Spin}

MRI is based on the interaction of atomic nuclei with an external magnetic field, an effect which is commonly known as Nuclear Magnetic Resonance (NMR). Atomic nuclei are composed of protons and neutrons, which can both be classified as spin$1 / 2$ fermions. The spin is a relativistic quantum phenomenon, which is described by the quantum number $s$. According to the Pauli principle, only atoms with uneven nucleon number or with both an uneven number of protons and neutrons have a non-zero spin: ${ }^{1} \mathrm{H}(s=1 / 2),{ }^{13} \mathrm{C}(s=1 / 2),{ }^{14} \mathrm{~N}(s=1),{ }^{17} \mathrm{O}(s=5 / 2)$ [72, 73]. In the context of MRI the ${ }^{1} \mathrm{H}$-isotope is of particular interest as it constitutes a building block for water and fat molecules, which occur abundantly in the human body.

Quantum mechanics defines the spin by means of the spin operator

$$
\hat{\boldsymbol{S}}=\left(\hat{S}_{x}, \hat{S}_{y}, \hat{S}_{z}\right),
$$

which satisfies the eigen-relations

$$
\begin{gathered}
\hat{\boldsymbol{S}}^{2}|\chi\rangle=\hbar^{2} s(s+1)|\chi\rangle, \\
\hat{S}_{z}|\chi\rangle=\hbar s_{z}|\chi\rangle, \quad s_{z}=-s,-s+1, \ldots, s-1, s,
\end{gathered}
$$

where $\hbar$ is the reduced Planck constant and $|\chi\rangle$ represents a spin state in a twodimensional complex Hilbert space. As a spin-1/2 particle the ${ }^{1} \mathrm{H}$ nucleus can occupy two eigenstates given by $|\uparrow\rangle$ (spin-up, $s_{z}=+1 / 2$ ) and $|\downarrow\rangle$ (spin-down, $s_{z}=$ $-1 / 2$ ). Any spin state can therefore be composed by a superposition of these two basis functions,

$$
|\chi\rangle=c_{\uparrow}|\uparrow\rangle+c_{\downarrow}|\downarrow\rangle
$$


where $c_{\uparrow}$ and $c_{\downarrow}$ are complex numbers [70].

The spin shares many similarities with the classical angular momentum and, according to the quantum mechanical correspondence principle, induces the magnetic moment

$$
\hat{\boldsymbol{\mu}}=\gamma \hat{\boldsymbol{S}} \text {. }
$$

The gyromagnetic ratio $\gamma$ is characteristic for each particle and takes the value $\gamma / 2 \pi=42.5774 \mathrm{MHz} \mathrm{T}^{-1}$ for ${ }^{1} \mathrm{H}$-isotopes [74].

\subsubsection{The Nuclear Zeeman Effect}

The energy of a spin in a constant and homogeneous external magnetic field, which for MRI is by convention defined to be parallel to the $z$-axis of the coordinate system,

$$
\boldsymbol{B}_{\mathrm{o}}=B_{\mathrm{o}} \boldsymbol{e}_{z}
$$

is described by the Hamiltonian

$$
\hat{H}=-\hat{\boldsymbol{\mu}} \cdot \boldsymbol{B}_{\mathrm{O}} \stackrel{\text { Eq.(2.5)(2.6) }}{=}-\gamma B_{\mathrm{O}} \hat{S}_{z} .
$$

Since this operator is time-independent, the eigenvalue equation of $\hat{H}$ coincides with the time-independent Schrödinger equation and the eigenvectors of $\hat{H}$ are given by those of $\hat{S}_{z}$, which for spin-1/2 particles yields

$$
\begin{aligned}
& E_{\uparrow}|\uparrow\rangle=\hat{H}|\uparrow\rangle \stackrel{\text { Eq.(2.3)(2.7) }}{=}-\frac{\hbar}{2} \gamma B_{0}|\uparrow\rangle, \\
& E_{\downarrow}|\downarrow\rangle=\hat{H}|\downarrow\rangle \stackrel{\text { Eq.(2.3)(2.7) }}{=}+\frac{\hbar}{2} \gamma B_{0}|\downarrow\rangle .
\end{aligned}
$$

This shows that the energy levels $E_{\uparrow}$ and $E_{\downarrow}$ of the corresponding eigenstates split up due to the coupling of the spin with the external magnetic field strength $B_{0}$. This phenomenon is frequently referred to as the nuclear Zeeman effect. The corresponding energy difference is given by

$$
\Delta E=\hbar \gamma B_{0}=\hbar \omega_{0},
$$

where

$$
\omega_{\mathrm{o}}:=\gamma B_{\mathrm{o}}
$$

is called Larmor frequency [75].

Using the quantum mechanical density operator formalism, the time evolution of the magnetic moment's expectation value in a potentially time-varying external magnetic field $\boldsymbol{B}(t)$ can shown to be determined by [76]

$$
\frac{\mathrm{d}}{\mathrm{d} t}\langle\hat{\boldsymbol{\mu}}\rangle=\gamma\langle\hat{\boldsymbol{\mu}}\rangle \times \boldsymbol{B}(t)
$$


Hence, the expectation value of a spin's magnetic moment $\langle\hat{\boldsymbol{\mu}}\rangle$ evolves according to the classical equation for the precession of a magnetic moment in an external magnetic field. For $\boldsymbol{B}(t)=\boldsymbol{B}_{0}$ the precession frequency is given by the Larmor frequency $\omega_{0}=\gamma B_{0}$ (Equation (2.11)) which is likewise related to the transition energy $\Delta E=\hbar \omega_{\mathrm{o}}$ (Equation (2.10)) between the two Zeeman states $|\uparrow\rangle$ and $|\downarrow\rangle$.

\subsubsection{Bulk Magnetization}

In MRI we are not interested in the behavior of individual spins but in the property of the aggregate ensemble of spins inside a voxel. We therefore introduce the magnetization operator $\hat{\boldsymbol{m}}$ as the integral of the magnetic moment operator $\hat{\boldsymbol{\mu}}$ weighted by the proton density $\rho(\boldsymbol{r})$ at position $\boldsymbol{r}$ inside a voxel volume $\mathcal{V}$,

$$
\hat{\boldsymbol{m}}=\frac{\int_{\mathcal{V}} \rho(\boldsymbol{r}) \hat{\boldsymbol{\mu}} d^{3} \boldsymbol{r}}{\mathcal{V}}
$$

At room temperature, the Fermi-Dirac statistics for fermions approach the Boltzmann distribution which determines the population of the energy states to be

$$
\begin{aligned}
& P_{\uparrow}=\frac{e^{-E_{\uparrow} / k_{B} T_{\mathrm{K}}}}{e^{-E_{\uparrow} / k_{B} T_{\mathrm{K}}}+e^{-E_{\downarrow} / k_{B} T_{\mathrm{K}}}}, \\
& P_{\downarrow}=\frac{e^{-E_{\downarrow} / k_{B} T_{\mathrm{K}}}}{e^{-E_{\uparrow} / k_{B} T_{\mathrm{K}}}+e^{-E_{\downarrow} / k_{B} T_{\mathrm{K}}}},
\end{aligned}
$$

with the Boltzmann constant $k_{B}$ and temperature $T_{K}$ [77]. Interestingly, for ${ }^{1} \mathrm{H}$ nuclei at $T_{\mathrm{K}}=293 \mathrm{~K}$ and an external magnetic field strength of $B_{0}=3 \mathrm{~T}$, which are common conditions in a clinical setting, the lower energy state $|\uparrow\rangle$ is only slightly more populated by a relative fraction of

$$
\frac{\Delta N}{N}=P_{\uparrow}-P_{\downarrow} \approx 10^{-5},
$$

with $N$ being the total number of spins. In the thermodynamic equilibrium, this disparity in the occupation of states effects a non-vanishing value for the magnetization, which points in the direction of the external magnetic field and is given by [68, 76]

$$
\langle\hat{\boldsymbol{m}}\rangle_{\mathrm{eq}}=\rho_{\mathcal{V}} \frac{\hbar^{2} \gamma^{2} B_{\mathrm{o}}}{4 k_{B} T} \boldsymbol{e}_{z}:=\left\langle\hat{m}_{z}\right\rangle_{\mathrm{eq}} \boldsymbol{e}_{z}
$$

with the spin density of the voxel

$$
\rho_{\mathcal{V}}:=\frac{\int_{\mathcal{V}} \rho(\boldsymbol{r}) d^{3} \boldsymbol{r}}{\mathcal{V}} .
$$




\subsubsection{Bloch Equation}

Measuring the magnetization $\langle\hat{\boldsymbol{m}}\rangle$ is the principle goal of all MRI experiments. The magnetization does not only contain information about the proton spin density of a voxel, see Equation (2.17), but can also be used to determine specific tissue properties, such as relaxation times, and to encode e.g. flow or diffusion information. However, the magnetization is not accessible to a direction measurement. Therefore, the fundamental idea of MRI is to tip the magnetization away from its equilibrium by applying a time-varying magnetic field by means of a resonant RF pulse

$$
\boldsymbol{B}_{1}^{\omega}(t)=B_{1}(t)\left(\begin{array}{c}
\cos (\omega t) \\
-\sin (\omega t) \\
0
\end{array}\right) .
$$

This principle is also know as spin excitation in the literature. The RF pulse possesses two main parameters: The carrier frequency $\omega$ and the envelope function $B_{1}(t)$, which will be discussed in more detail later.

In general, the evolution of the magnetization $\langle\hat{\boldsymbol{m}}\rangle=\left(\left\langle\hat{m}_{x}\right\rangle,\left\langle\hat{m}_{y}\right\rangle,\left\langle\hat{m}_{z}\right\rangle\right)^{\mathrm{T}}$ in an external magnetic field is governed by the Bloch Equation [78]

$$
\frac{\mathrm{d}}{\mathrm{d} t}\langle\hat{\boldsymbol{m}}\rangle=\gamma\langle\hat{\boldsymbol{m}}\rangle \times \boldsymbol{B}(t)+\left(\begin{array}{c}
-\frac{1}{T_{2}}\left\langle\hat{m}_{x}\right\rangle \\
-\frac{1}{T_{2}}\left\langle\hat{m}_{y}\right\rangle \\
\frac{1}{T_{1}}\left(\left\langle\hat{m}_{z}\right\rangle_{\mathrm{eq}}-\left\langle\hat{m}_{z}\right\rangle\right)
\end{array}\right),
$$

where $\left\langle\hat{m}_{z}\right\rangle_{\mathrm{eq}}$ is defined by Equation (2.17) and $\boldsymbol{B}(t)$ is the total external magnetic field. The Bloch Equation extends the dynamical behavior given in Equation (2.12) by a phenomenological term, which accounts for relaxation effects quantified by the $T_{1}$ and $T_{2}$ relaxation constants. $T_{1}$ models the spin-lattice relaxation and characterizes the time for $\left\langle\hat{m}_{z}\right\rangle$ to recover the equilibrium value $\left\langle\hat{m}_{z}\right\rangle_{\mathrm{eq}}$ after excitation. $T_{2}$ accounts for spin-spin relaxation and quantifies the decay of the transverse magnetization $\left\langle\hat{m}_{x y}\right\rangle$, which is commonly represented in the complex plain by

$$
\left\langle\hat{m}_{x y}\right\rangle:=\left\langle\hat{m}_{x}\right\rangle+i\left\langle\hat{m}_{y}\right\rangle \text {. }
$$

Solving the Bloch Equation leads to two important findings. First, a resonant RF pulse $\boldsymbol{B}_{1}^{\omega}(t)$ tips the magnetization vector $\langle\hat{\boldsymbol{m}}\rangle$ away from its longitudinal equilibrium, which yields a non-zero transverse component $\left\langle\hat{m}_{x y}\right\rangle$. Second, for external fields with vanishing transverse components, i.e. $B_{x}=0$ and $B_{y}=0$, the Bloch Equation implies the solution

$$
\begin{gathered}
\left\langle\hat{m}_{z}\right\rangle(\boldsymbol{r}, t)=\left\langle\hat{m}_{z}\right\rangle_{\mathrm{eq}}+\left(\left\langle\hat{m}_{z}\right\rangle(\boldsymbol{r}, \mathrm{o})-\left\langle\hat{m}_{z}(\boldsymbol{r})\right\rangle_{\mathrm{eq}}\right) e^{-t / T_{1}(\boldsymbol{r})}, \\
\left\langle\hat{m}_{x y}\right\rangle(\boldsymbol{r}, t)=\left\langle\hat{m}_{x y}\right\rangle(\boldsymbol{r}, \mathrm{o}) e^{-t / T_{2}(\boldsymbol{r})} e^{-i \int_{\mathrm{o}}^{t} \gamma B_{z}(\boldsymbol{r}, \tau) d \tau} .
\end{gathered}
$$


For the constant field $\boldsymbol{B}(t)=\boldsymbol{B}_{\mathrm{o}}=B_{\mathrm{o}} \boldsymbol{e}_{z}$ the integral in Equation (2.23) yields

$$
\int_{0}^{t} \gamma B_{z}(\boldsymbol{r}, \tau) d \tau=\int_{0}^{t} \gamma B_{0} d \tau=\gamma B_{0} t \stackrel{\text { Eq.(2.11) }}{=} \omega_{0} t
$$

Hence, the coupling of the transverse magnetization $\left\langle\hat{m}_{x y}\right\rangle$ with $\boldsymbol{B}_{\mathrm{o}}$ causes a precession of $\left\langle\hat{m}_{x y}\right\rangle$ with Larmor frequency $\omega_{0}$ around the $z$-axis until $\left\langle\hat{m}_{x y}\right\rangle$ vanishes through relaxation. It is a physical principle that a precessing magnetization emits an electro-magnetic wave, which is the signal acquired in MRI experiments.

\subsubsection{Conclusion}

MRI is a highly complex process based on the manipulation of a quantum mechanical system using external magnetic fields. Still, the key idea behind MRI experiments can be summarized in three simple steps: (I) A strong external magnetic field induces a bias in the originally isotropic nuclear spin distribution of tissue, which results in a net magnetization. (II) This magnetization can be tipped away from its equilibrium into the transverse plane using a resonant RF pulse. (III) The signal emitted by the precessing transverse magnetization is acquired and contains tissue specific information.

\subsection{THE PULSED NMR EXPERIMENT}

In this section, we will illustrate how the fundamental principles of MRI are exploited in practical experiments to generate images. We will start off with the description of MRI hardware components. After introducing the concept of slice excitation, we will expound on the ideas of spatial encoding and k-space sampling, which naturally suggests the underlying idea behind image reconstruction in MRI [79].

\subsubsection{The MRI System}

A modern MRI system consists of six major elements: (I) A main magnet coil to create a strong external magnetic field which is supposed to be as homogeneous as possible. (II) Gradient coils for all three axis which enable the spatial encoding of the signal. (III) A transmit- and receive RF system for spin excitation and signal acquisition. (IV) Shielding, to reduce the outreach of the external magnetic field. (V) A shimming system to ensure the uniformity of the external magnetic field. (VI) A computer to control data acquisition and image reconstruction. A schematic of elements (I)-(III) is depicted in Figure 2.1. 


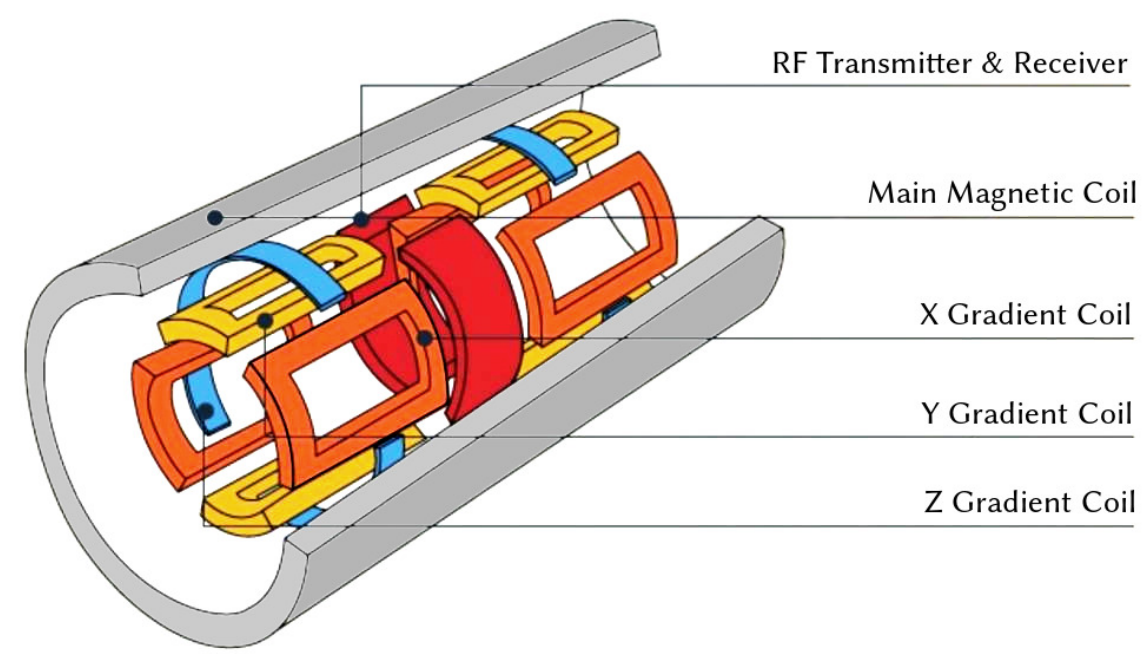

Figure 2.1.: Schematic of the MRI coil system. The main magnetic coil creates the external magnetic field. The $x, y$ and $z$ gradient coils enable the spatial encoding of the signal. The RF transmitter and receiver are used for spin excitation and signal acquisition. The graphic is adapted from [8o].

\subsubsection{Slice-Selective Excitation}

In MRI experiments we desire a spatially resolved image of a specific Region of Interest (ROI). To avoid disturbing signals from other parts of the body, it is possible to excite only spins from a certain predefined region by making use of the spectral nature of spin excitation. One of the most common approaches is the slice-selective excitation.

As we can learn from Equation (2.11), the Larmor frequency of spins, or similarly of the bulk magnetization vector, depends on the strength of the external magnetic field. The magnetization vector can be tipped away from its equilibrium when a resonant RF pulse is applied. The simple yet powerful idea which enables selective excitation is to modulate the resonance frequency of the magnetization vector using an additional linear magnetic field gradient $G$. This allows for the excitation of a subset region by applying an RF pulse with accordingly band-limited frequency spectrum.

The magnetic field gradient

$$
G:=G e_{\mathrm{s}}
$$

yields an additional magnetic field

$$
B_{\mathrm{S}}(\boldsymbol{r})=(\boldsymbol{G} \cdot \boldsymbol{r}) \boldsymbol{e}_{z}
$$


which points in $z$-direction and whose strength depends on the spatial position $\boldsymbol{r}$. In combination with the constant external magnetic field $\boldsymbol{B}_{\mathrm{o}}$ the total magnetic field is in good approximation ${ }^{1}$ given by

$$
\boldsymbol{B}(\boldsymbol{r})=\boldsymbol{B}_{\mathrm{o}}+\boldsymbol{B}_{\mathrm{s}}(\boldsymbol{r}) \stackrel{\mathrm{Eq} \cdot(2.6)(2.26)}{=}\left(B_{\mathrm{o}}+\boldsymbol{G} \cdot \boldsymbol{r}\right) \boldsymbol{e}_{z}:=B(\boldsymbol{r}) \boldsymbol{e}_{z}
$$

Accordingly, the Larmor frequency $\omega_{\mathrm{L}}$ becomes spatially dependent,

$$
\omega_{\mathrm{L}}(\boldsymbol{r}) \stackrel{\text { Eq.(2.11) }}{=} \gamma B(\boldsymbol{r}) \stackrel{\text { Eq.(2.27)(2.11) }}{=} \omega_{\mathrm{O}}+\gamma \boldsymbol{G} \cdot \boldsymbol{r} .
$$

Hence, the magnetic field gradient $G$ introduces a linear frequency variation

$$
\Delta \omega_{\mathrm{L}}=\gamma G \Delta s
$$

along a distance $\Delta s$ in direction of $e_{\mathrm{s}}$.

Let the desired slice of thickness $\Delta s$ be perpendicular to $e_{\mathrm{S}}$ and let $\boldsymbol{r}_{\mathrm{c}}$ denote the center of the slice. Then, the Larmor frequency at the slice center is given by

$$
\omega_{\mathrm{L}}\left(\boldsymbol{r}_{\mathrm{c}}\right) \stackrel{\mathrm{Eq} \cdot(2.28)}{=} \omega_{\mathrm{o}}+\gamma \boldsymbol{G} \cdot \boldsymbol{r}_{\mathrm{c}}:=\omega_{\mathrm{c}} .
$$

The magnetization inside this slice can be excited exclusively using an RF pulse $\boldsymbol{B}_{1}^{\omega}(t)$ (Equation (2.19)) with carrier frequency $\omega=\omega_{\mathrm{c}}$ and frequency bandwidth $\Delta \omega_{\mathrm{L}}$.

With the definition of the shifted Larmor frequency

$$
\Omega(\boldsymbol{r}):=\omega_{\mathrm{L}}(\boldsymbol{r})-\omega_{\mathrm{C}}
$$

the excitation profile for small flip angles ${ }^{2}$ is given by [81]

$$
\theta(\boldsymbol{r}) \approx \gamma\left|\int_{-\infty}^{\infty} B_{1}(\tau) e^{i \Omega(\boldsymbol{r}) \tau} d \tau\right| .
$$

Here, the polar flip angle $\theta$ describes the degree to which the magnetization vector is tipped away from the $z$-axis into the transverse plane. Equation (2.32) states that the excitation profile can be approximated by the Fourier transform of the RF pulse envelope $B_{1}(t)$. Hence, to accomplish a rectangular excitation profile of bandwidth $\Delta \omega_{\mathrm{L}}$ the pulse envelope should be of type

$$
B_{1, \operatorname{sinc}}^{\Delta \omega_{L}}(t)=\operatorname{sinc}\left(\frac{\Delta \omega_{\mathrm{L}}}{2} t\right)
$$

1 An inhomogeneous static magnetic field cannot have a varying field strength along a single axis only, but requires so called concomitant fields along the remaining axis to fulfill the Maxwell equations. However, these additional components are small and can be neglected for high magnetic field strengths in the order of several Tesla, which is the case in the setting of this thesis [81]. Still, in low-field MRI the concomitant fields should be accounted for as they can lead to artifacts [82].

2 This approximation holds well for flip angles up to $\theta=30^{\circ}$ and is still acceptable for flip angles smaller than $\theta=90^{\circ}$. 
For practical reasons, the RF pulse duration is restricted to a finite duration $T_{\mathrm{RF}}$. To avoid strong side-lobes and to ensure a reasonable excitation profile, functions such as the Hann window $W_{\mathrm{H}}(t)$ are commonly used to apodize the sinc-pulse [81],

$$
B_{1}^{\Delta \omega_{L}}(t)=W_{\mathrm{H}}(t) B_{1, \operatorname{sinc}}^{\Delta \omega_{L}}(t)=\frac{1}{2}\left(1+\cos \left(\frac{2 \pi t}{T_{\mathrm{RF}}}\right)\right) \operatorname{sinc}\left(\frac{\Delta \omega_{\mathrm{L}}}{2} t\right)
$$

Figure 2.2 illustrates the effect of RF pulse truncation and apodization. While an infinite sinc pulse possesses a rectangular frequency domain representation, the Fourier transform of a cropped sinc pulse shows severe side-lobes. These sidelobes can significantly be reduced by apodizing the sinc function [63].
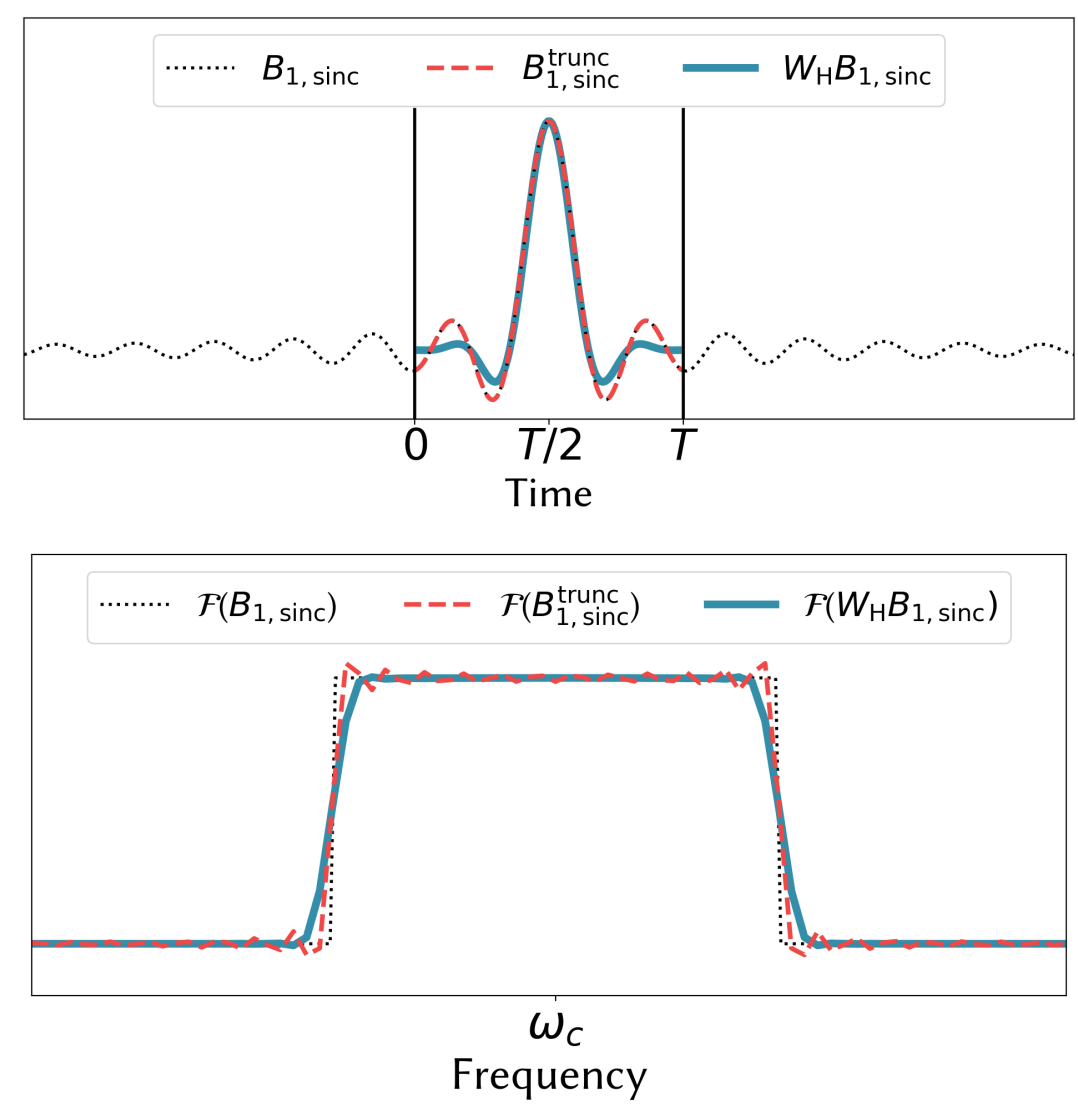

Figure 2.2.: Illustration of the effect of RF pulse truncation and apodization in time and frequency domain. (Top) Infinite sinc, truncated sinc and Hann-windowed sinc RF pulse envelopes in time domain. The vertical lines imply the finite pulse duration. (Bottom) Real part of the Fourier transformed $(\mathcal{F})$ RF pulses in frequency domain, where the shift according to the carrier frequency is implicitly assumed. 
To comply with the general notation in MRI literature and without loss of generality, we choose the slice-selection gradient to apply along the $z$-axis. To simplify further discussions, we introduce the notation

$$
\boldsymbol{B}_{1}^{\Delta \omega_{L}, \omega_{c}}(t) \stackrel{\text { Eq. (2.19)(2.34) }}{=} B_{1}^{\Delta \omega_{L}}(t)\left(\begin{array}{c}
\cos \left(\omega_{c} t\right) \\
-\sin \left(\omega_{c} t\right) \\
0
\end{array}\right):=B_{\mathrm{rf}}\left(z_{c}, \Delta z\right)
$$

for an RF pulse, which - with a particular choice of the slice-selection gradient, the bandwidth $\Delta \omega_{L}$ and the carrier frequency $\omega_{c}$ - excites a slice of thickness $\Delta z$ with center position $z_{c}$. Note that this convention evokes a vanishing $z$-component, for which Equation (2.35) can also be expressed using a complex-valued shorthand notation

$$
\boldsymbol{B}_{1, x y}^{\Delta \omega_{L}, \omega_{c}}(t):=\boldsymbol{B}_{1, x}^{\Delta \omega_{L}, \omega_{c}}(t)+i \boldsymbol{B}_{1, y}^{\Delta \omega_{L}, \omega_{c}}(t)=B_{1}^{\Delta \omega_{\mathrm{L}}}(t) e^{-i \omega_{c} t} .
$$

A schematic illustration of the quantities involved in slice-selection is depicted in Figure 2.3.

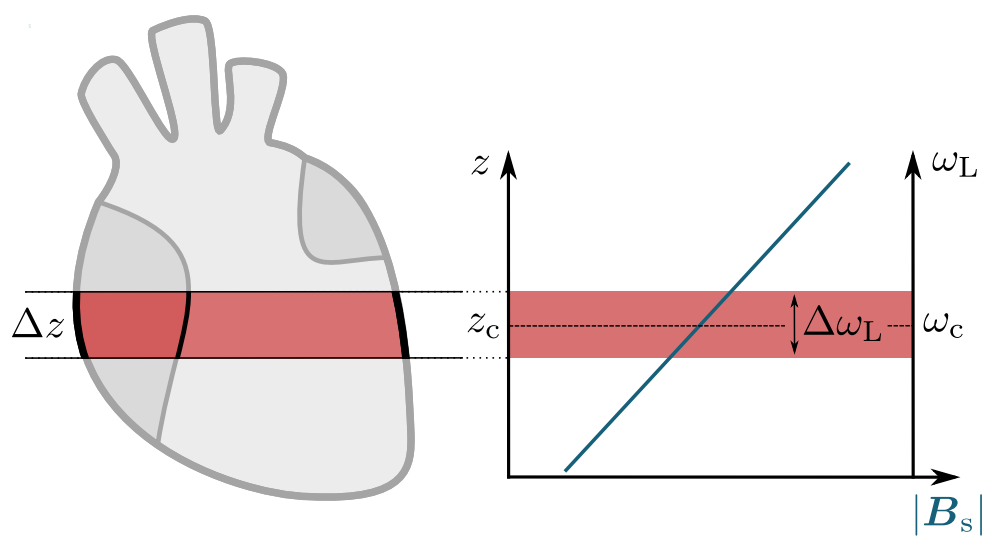

Figure 2.3:: Schematic illustration of the quantities involved in slice-selection: Center of slice $\left(z_{\mathrm{c}}\right)$, slice thickness $(\Delta z)$, Larmor frequency at the center of slice $\left(\omega_{\mathrm{c}}\right)$, Larmor frequency variation inside of the slice $\left(\Delta \omega_{\mathrm{L}}\right)$, magnitude of the magnetic field induced by the slice-selection gradient $\left(\left|\boldsymbol{B}_{\mathrm{s}}\right|\right)$.

\subsubsection{Spatial Encoding}

Magnetic field gradients do not only enable slice-selective excitation but can also be utilized for spatial encoding. Given the external magnetic field with applied magnetic field gradient (Equation (2.27)) the evolution of the transverse magnetization $\left\langle\hat{m}_{x y}\right\rangle$ according to the Bloch Equation (2.20) is given by Equation (2.23) and reads

$$
\left\langle\hat{m}_{x y}\right\rangle(\boldsymbol{r}, t)=\left\langle\hat{m}_{x y}\right\rangle(\boldsymbol{r}, \mathrm{o}) e^{-t / T_{2}(\boldsymbol{r})} e^{-i\left(\omega_{0} t+\varphi(\boldsymbol{r}, t)\right)} .
$$


The phase term $\varphi(r, t)$ is determined by the time course of the magnetic field gradient

$$
\varphi(\boldsymbol{r}, t):=\boldsymbol{r} \cdot \int_{0}^{t} \gamma \boldsymbol{G}(\tau) d \tau:=\boldsymbol{r} \cdot \boldsymbol{k}(t)
$$

with the $k$-space trajectory defined by

$$
\boldsymbol{k}(t):=\int_{0}^{t} \gamma \boldsymbol{G}(\tau) d \tau
$$

In MRI experiments, magnetization of the entire excited region $V$ contributes to the total acquired signal $S$, which can be modeled by the integral

$$
\int_{V}\left\langle\hat{m}_{x y}\right\rangle(\boldsymbol{r}, t) d \boldsymbol{r}
$$

Omitting the carrier frequency term $e^{-i \omega_{0} t}$, which is removed by quadrature demodulation in practice, and the relaxation term $e^{-t / T_{2}}$, which is negligibly small for our applications, we yield the signal equation

$$
S(\boldsymbol{k}) \stackrel{\text { Eq.(2.37)(2.38) }}{=} \int_{V}\left\langle\hat{m}_{x y}\right\rangle(\boldsymbol{r}, \mathrm{o}) e^{-i \boldsymbol{r} \cdot \boldsymbol{k}(t)} d \boldsymbol{r} \text {. }
$$

Equation (2.41) suggests a Fourier relation between the transverse magnetization $\left\langle\hat{m}_{x y}\right\rangle$ in image domain and the acquired signal $S$ in k-space, i.e. the spatial frequency domain. The Fourier relation Equation (2.41) is not only the basis for image reconstruction in MRI, which will be covered in more detail in Section 2.3.1, but furthermore imposes an important sampling requirement on the data acquisition.

In MRI experiments it is not possible to acquire the signal continuously, thus only discrete samples of a finite area of k-space can be obtained. The Nyquist sampling theorem states that a discretely sampled signal with sampling interval $\Delta k$ is replicated in its Fourier conjugate domain at intervals $1 / \Delta k$. To prevent aliasing, often called wrap-around-, infold- or overlapping-artifacts, the sampling interval $\Delta k$ should be chosen such that it fulfills the Nyquist condition

$$
\Delta k \leq \frac{1}{\mathrm{FOV}^{\prime}}
$$

with a Field of View (FOV) larger than the extend of the imaged object. Likewise, the spatial resolution of the reconstructed image is given by

$$
\Delta x:=\frac{\text { FOV }}{N} \stackrel{\text { Eq.(2.42) }}{=} \frac{1}{N \Delta k},
$$

with $N$ being the number of acquired samples [81]. An acquisition is called fully sampled, if the Nyquist condition is fulfilled. To spare acquisition time, it is generally desirable to perform undersampled acquisitions, i.e. to acquire less data then demanded by the Nyquist condition. In Section 2.3.1 we will present the concepts of parallel imaging and compressed sensing which circumvent the Nyquist condition and allow for suitable reconstructions even for high undersampling factors. 


\subsection{4 k-space Trajectory}

Closely related to the sampling requirement discussed in the previous section is the k-space trajectory (Equation (2.39)), which constitutes an important concept for the design of MRI experiments. Its specific choice can have a strong impact on the resulting image quality. In general, the k-space should efficiently be covered by the trajectory, such that sufficient spatial frequency information is acquired to fulfill the Nyquist condition stated in Equation (2.42), which then allows for proper image reconstruction. The most common trajectories are depicted in Figure 2.4.

(a) $\mathrm{EPI}$

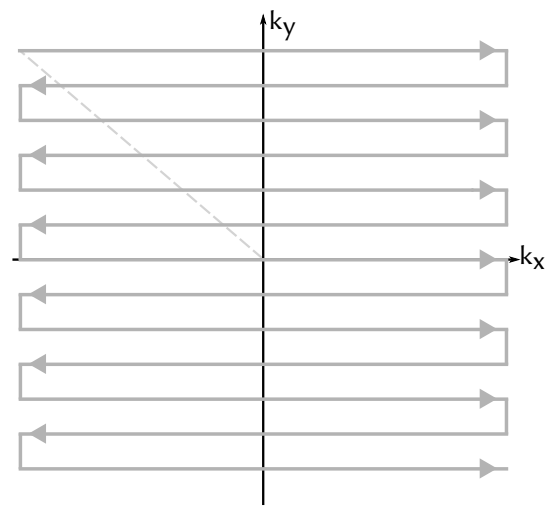

(c) Cartesian

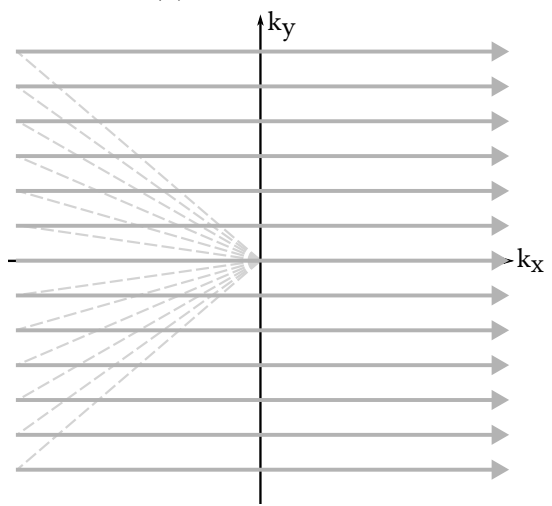

(b) Spiral

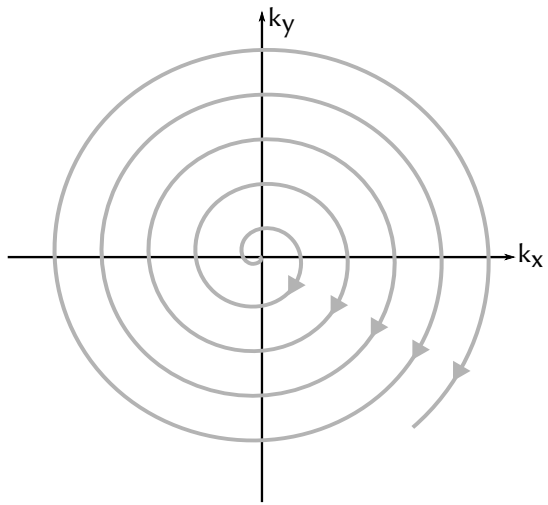

(d) Radial

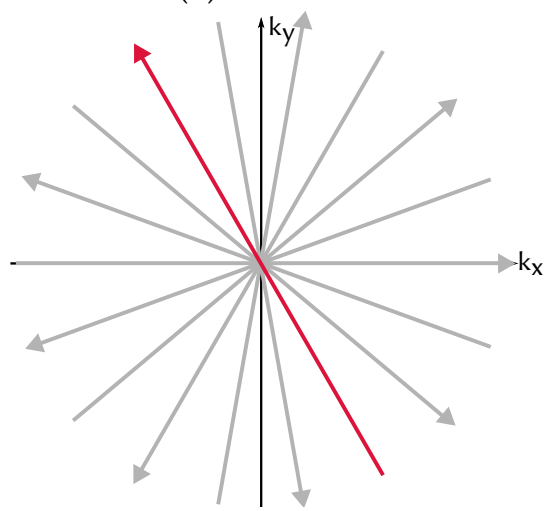

Figure 2.4.: Schematic of popular k-space trajectories: (a) EPI, (b) Spiral, (c) Cartesian, (d) Radial. The little arrow-heads point in the read-out direction, the dashed lines denote the trajectory course where no data is acquired. A schematic of the gradients used to acquire the red spoke highlighted in (d) can be found in Figure 2.5.

Very long trajectories, that aim to cover the entire k-space within a single excitation as in EPI Figure 2.4a or spiral imaging Figure $2.4 \mathrm{~b}$ are very efficient and therefore allow for a high temporal resolution [32, 83]. However, for long read-out 
times relaxation effects can non longer be neglected and off-resonances caused by field inhomogeneities can lead to image blurring and distortions [84].

To avoid this problem, the k-space signal can be acquired using multiple shorter read-out lines, which is achieved by applying a sequence of RF excitations, also called shots, and read-out gradients. The by far most common strategy is the Cartesian trajectory Figure 2.4c. Here, a single line is acquired per shot while a phase encoding determines the line's offset from the k-space center. In each shot the trajectory traverses k-space in the same read-out direction, which makes this approach resilient towards system imperfections such as gradient delays [85]. Furthermore, the data is naturally sampled on a rectilinear (Cartesian) grid, which enables efficient image reconstruction using the Fast Fourier Transform (FFT). However, samples in phase encoding direction are temporally far apart, which makes Cartesian trajectories sensitive to motion artifacts such as ghosting [86].

An interesting alternative to Cartesian imaging is the radial trajectory shown in Figure 2.4d. Here, the read-out lines cross the center of k-space and are referred to as spokes. Each spoke captures an equal amount of low and high spatial frequencies, which constitutes a consistent sampling. The redundant information contained in the intrinsically oversampled k-space center induces an averaging effect, which can balance errors and inconsistencies from individual spokes and makes radial imaging robust to motion artifacts [87]. The frequently sampled k-space center can additionally be used to detect motion, which can be exploited for self-gating as will be explained in Section 2.5.3. Moreover, radial imaging can provide a satisfactory image quality even for a very little number of acquired spokes, since streaking artifacts evoked by k-space undersampling are less disturbing compared to Cartesian trajectories, where omitted lines lead to reduced resolution or corrupting aliasing artifacts. Nevertheless, one of the major drawbacks of radial imaging is its aptness to system imperfections, which will be further discussed in Section 2.3.3. A detailed examination of the advantages and disadvantages of radial imaging can be found in [88].

Typically for all trajectories, the MRI system internally doubles the sampling rate of the receiver to reduce the sampling distance $\Delta k$ on the k-space lines. For radial trajectories, this approach naturally increases the FOV in both spatial directions by a factor of two, which contributes to the fulfillment of the Nyquist condition Equation (2.42) and prevents aliasing artifacts. In contrast, for Cartesian imaging only the read-out direction benefits from this so called read-out oversampling, while the reduction of the sampling distance in phase encoding direction requires additional line scans [88]. 


\subsubsection{Gradient-Echo Sequences}

The time course of $\boldsymbol{k}(t)$ is given by Equation (2.39) and therefore is ruled by the switching of external magnetic field gradients $G$. Additional gradients and an RF pulse are required for slice-selective excitation as well as the generation of specific tissue contrasts. The succession of pulses and gradients is commonly visualized in a pulse diagram, which serves as a blueprint for the acquisition of a k-space line. In an actual pulse sequence, such a unit is repeatedly executed while gradient strengths and pulse characteristics may vary to achieve the desired k-space trajectory and to induce a certain contrast.

(a) FLASH

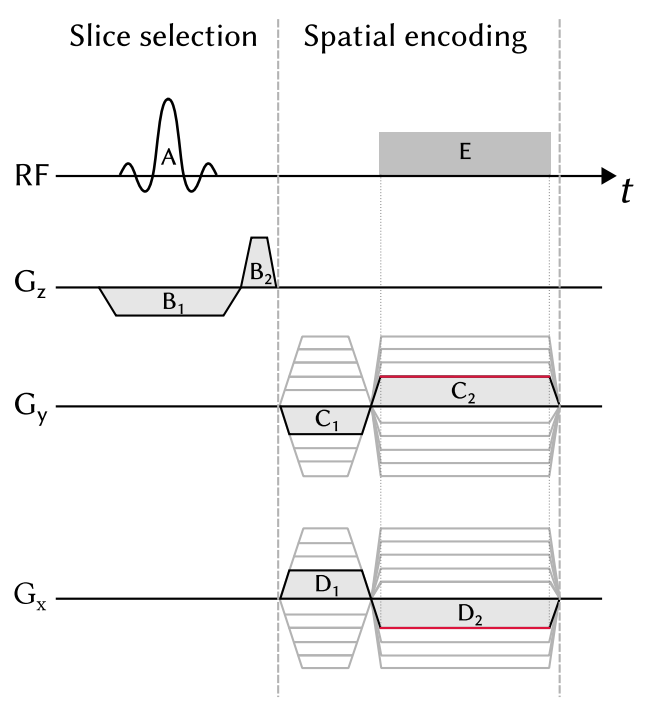

(a) bSSFP

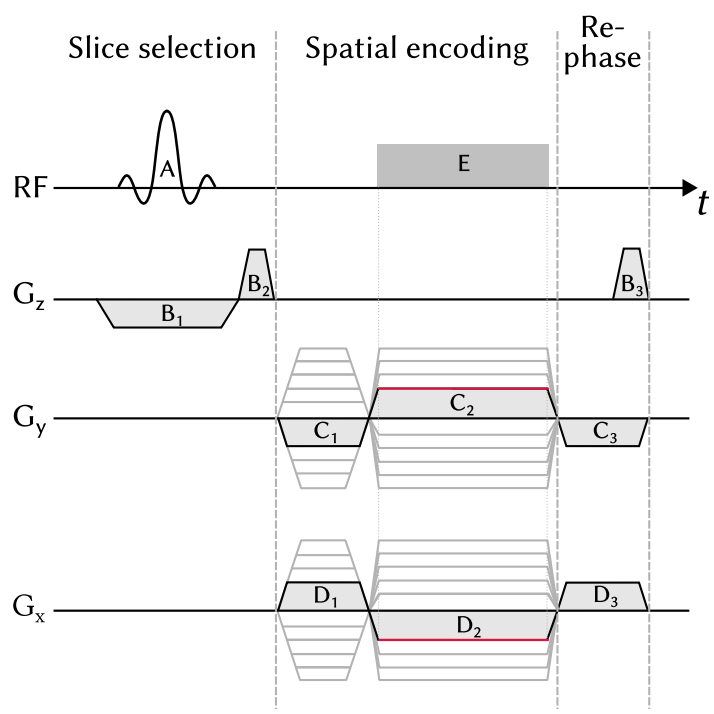

Figure 2.5.: Pulse diagram for a radial FLASH (a) and a radial balanced Steady-State Free Precession (bSSFP) (b) sequence. The RF pulse $(A)$ and gradient $\left(B_{1}\right)$ are used for slice-selection and the rewinder-gradient $B_{2}$ compensates for the corresponding dephasing. Gradients $\left(C_{1}\right)$ and $\left(D_{1}\right)$ guide the k-space trajectory out of the k-space center. Read-out gradients $\left(C_{2}\right)$ and $\left(D_{2}\right)$ make the trajectory traverse the k-space through its center. During the flat-top time of the read-out gradients, the signal is acquired $(E)$. In (b), the gradients $\left(B_{3}\right),\left(C_{3}\right)$ and $\left(D_{3}\right)$ balance the zeroth gradient moment of each axis to zero. The red lines highlight the gradient strength used to acquire a radial spoke, which is schematically depicted in Figure $2.4 \mathrm{~d}$. Without loss of generality and to follow the general notation of MRI literature, the slice-selective gradient is applied along the $z$-axis. To save time, the gradients $\left(B_{2}\right),\left(C_{1}\right)$ and $\left(D_{1}\right)$ are commonly played simultaneously.

For fast imaging, so called gradient-echo sequences - as opposed to spin-echo sequences, which will not be covered here - are the method of choice, with the FLASH sequence being one of the most popular [12]. Figure 2.5 shows the pulse diagram 
of a single-slice FLASH sequence with radial read-out. Strategies for multi-slice and volumetric imaging will be covered in Section 2.5.1. Slice-selective excitation is achieved by the joint activation of an RF pulse $(A)$ and a gradient pulse $\left(B_{1}\right)$, see Section 2.2.2. The rewinder gradient $\left(B_{2}\right)$ compensates for the phase evolution caused by the slice-selection gradient $\left(B_{1}\right)$. The radial trajectory for spatial encoding is accomplished by the simultaneous activation of in-plane read-out gradients: The dephasing gradients $\left(C_{1}\right)$ and $\left(D_{1}\right)$ guide the trajectory out of the k-space center. The actual data acquisition $(E)$, during which the trajectory traverses the k-space on a straight line through the k-space center, happens exclusively during the flattop time of the read-out gradients $\left(C_{2}\right)$ and $\left(D_{2}\right)$. Additional spoiler gradients can be activated after the acquisition of a line to dephase residual transverse magnetization, which prevents signal interference with future read-outs [89]. However, the use of a randomized phase for the RF excitation pulse was shown to have a similar effect [90]. This renders spoiler gradients obsolete and thus allows for a shorter Repetition Time (TR), which defines the time span between the start of two consecutive pulse units. For further acceleration, the gradients $\left(B_{2}\right),\left(C_{1}\right)$ and $\left(D_{1}\right)$ are commonly played simultaneously.

Instead of destroying residual transverse magnetization by RF or gradient spoiling, the bSSFP sequence makes use of additional gradients $\left(B_{3}\right),\left(C_{3}\right)$ and $\left(D_{3}\right)$ to rephase the transverse magnetization [91]. These balancing gradients effect a vanishing zeroth gradient moment on all axis. A peculiarity of the bSSFP sequence is the fact that the Echo Time (TE), i.e. the time span between the center of the RF pulse $(A)$ and the center of acquisition $(E)$, must be half as long as the TR. In combination with a phase variation of $\pi$ in each RF pulse, effective magnetization rephasing can be achieved [89]. This rephasing of the gradients yields a higher Signal-to-Noise $R a$ tio (SNR) for the bSSFP compared to the FLASH sequence. Therefore and because of the superior blood to myocardium contrast, the bSSFP sequence is a particularly popular choice for cardiac MRI [11, 92]. However, the bSSFP is prone to system imperfections and, as a result of off-resonances, images often suffer from banding artifacts, which become more severe for higher magnetic field strengths [89]. Moreover, it demands a relatively high flip angle $\theta \gtrsim 35^{\circ}$ which can be problematic due to Specific Absorption Rate (SAR) limitations, especially for large volume excitations or long measurement times. In contrast, the FLASH sequence uses much smaller flip angles $\theta \lesssim 20^{\circ}$ and enables shorter TRs for dispensing with rephasing gradients. It is less prone to system imperfections and still provides a reasonable blood to myocardium contrast at $3 \mathrm{~T}$ field strength for single-slice imaging [42]. Nevertheless, the FLASH sequence is not suitable for volumetric measurements, as we will discuss in Section 2.5.1. 


\subsubsection{Conclusion}

The concepts of slice-selective excitation, spatial encoding and the k-space formalism are crucial for the understanding of any MRI experiment. In combination with a gradient-echo sequence, rudimentary MRI image generation and reconstruction can be performed. However, fast and robust multi-dimensional cardiac MRI demands for more advanced concepts.

\subsection{CONCEPTS OF MODERN IMAGE RECONSTRUCTION AND DATA COR- RECTION}

In this chapter, we will outline the concepts of parallel imaging and compressed sensing, which allow for a significant reduction of scan time. Moreover, we will describe the basics of non-Cartesian image reconstruction and outline the problem of gradient delays for radial imaging.

\subsubsection{Parallel Imaging and Compressed Sensing}

The fundamental idea of image reconstruction in MRI originates from signal equation (2.41) which formalizes the Fourier relation between the acquired k-space signal and the actual image information. Here, we will present key concepts in modern MRI image reconstruction by extending this signal equation to include multiple receive channels and by introducing the concept compressed sensing.

PARALLEL IMAGING To accelerate MRI scans and to improve the SNR, phased array coils are commonly utilized for parallel or simultaneous data acquisition, which coined the term parallel imaging [93, 94]. A phased array coil consists of various receive channels, each being sensitive to a certain spatial region, which can be modeled by a complex sensitivity map $c(r)$. A schematic to illustrate the concept of parallel imaging is provided in Figure 2.6.

Modifying Equation (2.41) accordingly yields the signal equation for coil $j$

$$
S_{j}(\boldsymbol{k})=\int_{V} c_{j}(\boldsymbol{r})\left\langle\hat{m}_{x y}\right\rangle(\boldsymbol{r}, \mathrm{o}) e^{-i \boldsymbol{r} \cdot \boldsymbol{k}(t)} d \boldsymbol{r} .
$$

If the data is available on a fully sampled Cartesian grid, the coil images $\left\langle\hat{m}_{x y}\right\rangle_{j}:=$ $c_{j}\left\langle\hat{m}_{x y}\right\rangle$ can efficiently be recovered by applying the FFT on each channel individually. One strategy to combine the resulting coil images is to calculate the Root-Sumof-Squares (RSS),

$$
\left\langle\hat{m}_{x y}\right\rangle_{\mathrm{RSS}}:=\sqrt{\sum_{j=1}^{N_{\mathrm{c}}}\left|\left\langle\hat{m}_{x y}\right\rangle_{j}\right|^{2}} .
$$




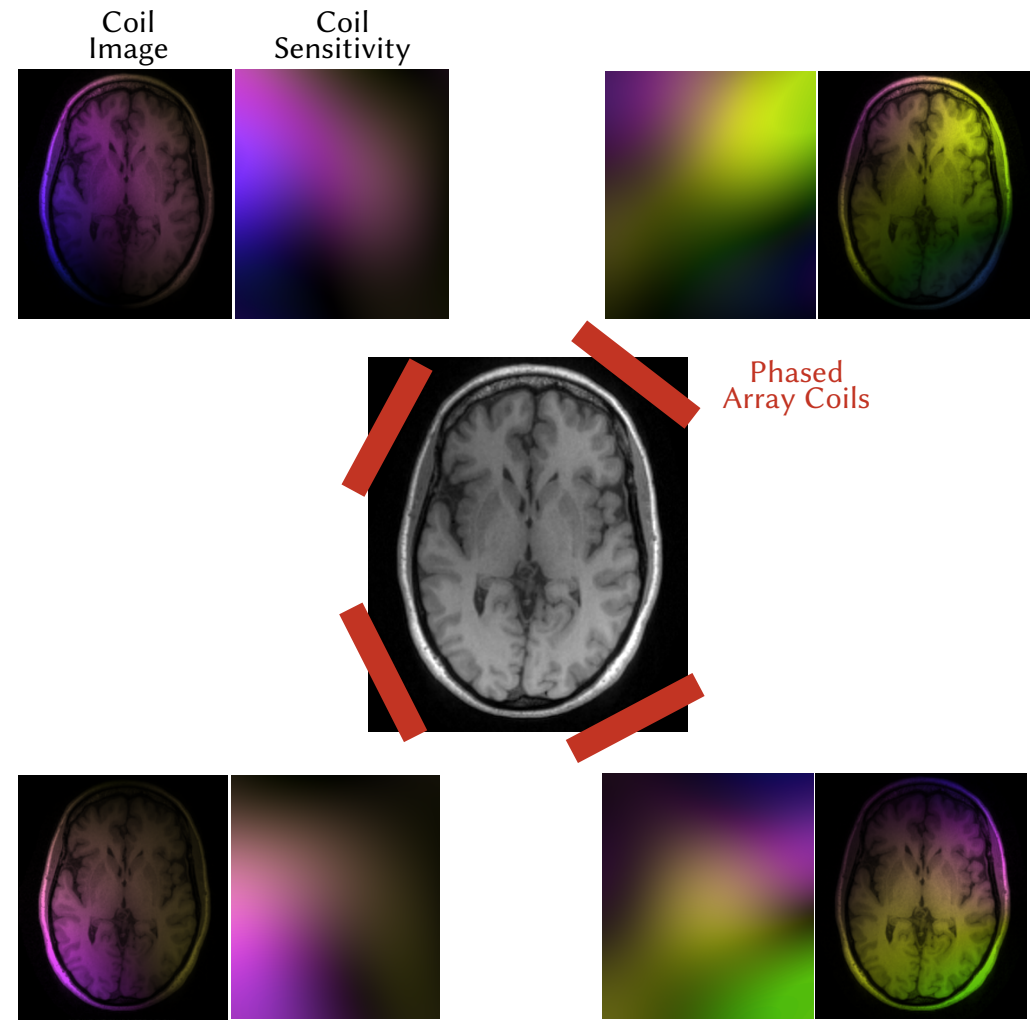

Figure 2.6.: Schematic illustration of the concept of parallel imaging. The actual object information is sensed by various phased array coils (indicated by the red rectangles). Each coil possesses a certain sensitivity, which covers only a portion of the object. Consequently, each coil observes only part of the object, which is indicated by the coil images. The additional information from multiple coils can be used to improve the SNR and to accelerate the data acquisition. The color in the images represents the complex phase.

However, a more accurate coil combination can be obtained using the MinimumVariance Unbiased Estimator (MVUE), which assumes equally distributed and independent Gaussian white noise [95],

$$
\left\langle\hat{m}_{x y}\right\rangle_{\mathrm{MVUE}}:=\frac{1}{\sum_{j=1}^{N_{\mathrm{c}}}\left|c_{j}\right|^{2}} \sum_{j=1}^{N_{\mathrm{c}}} c_{j}^{H}\left\langle\hat{m}_{x y}\right\rangle_{j},
$$

where ${ }^{H}$ denotes the Hermitian adjoint. For the latter approach, explicit knowledge of the coil sensitivity maps $c_{j}$ for each coil is required. The estimation of coil sensitivities is often referred to as coil calibration.

The Eigenvector-based Iterative Self-Consistent Parallel Imaging Reconstruction (ESPIRiT) method has proved to be one of the most powerful methods to determine accurate coil sensitivity maps and is widely used in the field of MRI [96]. ESPIRiT 
exploits local correlations in k-space to formulate a sub-space constraint on the sensitivity maps, which can then be recovered using a Singular Value Decomposition (SVD). To operate, it requires a fully sampled calibration region in the center of k-space with samples on a rectilinear grid. Therefore, non-Cartesian data must be gridded in a pre-processing step before they can be processed with ESPIRiT.

Another method to obtain high-quality coil sensitivity maps is NLINV which treats Equation (2.47) as a nonlinear inverse problem [40, 41]. In Chapter 5 we augment this algorithm to SMS-NLINV, which enables image reconstruction and coil calibration of multiple simultaneously acquired slices for Cartesian and nonCartesian trajectories. In Chapter 7 we use this technique in combination with Extended Nonlinear Inversion inspired by ESPIRiT (ENLIVE) [97] for high quality coil calibration of SMS and volumetric SOS measurements in self-gated cardiac MRI.

While the calculation of the MVUE in Equation (2.46) is seldomly used in practice, coil sensitivities play a crucial role when fast scan times are desired and thus only undersampled data is acquired. In fact, missing k-space data can be compensated by using the complementary spatial information contained in the receive coils, which allows for a significant increase in scan time [13, 93, 94]. To formalize this concept, image reconstruction in MRI is commonly posed as an inverse problem.

INVERSE PROBLEM FORMULATION Signal equation (2.44) can be reformulated using the operator-based vector notation

$$
\boldsymbol{y}=F \boldsymbol{x}+\boldsymbol{n},
$$

with the forward operator

$$
F:=P \mathcal{F} C \text {. }
$$

Here, $\boldsymbol{y}=\left(y_{1}, \ldots, y_{N_{c}}\right)^{T}$ is the vector of acquired discrete k-space samples for all $N_{c}$ coils. $P$ is the projection onto a certain k-space trajectory, $\mathcal{F}$ is the Fourier transform and $C$ is the coil sensitivity operator, which multiplies the vector of coil sensitivities $\boldsymbol{c}=\left(c_{1}, \ldots, c_{N_{c}}\right)$ with the image content $\boldsymbol{x}=\left\langle\hat{m}_{x y}\right\rangle$. We have furthermore included the term $n$, which describes the channel-wise corruption by complex Gaussian white noise [67]. For further considerations we assume noise in the channels to be uncorrelated and of equal variance. This assumption does not always hold true in actual phased array coils, but can be achieved using a pre-whitening step [98]. Note that the variables $\boldsymbol{x}$ and $\boldsymbol{y}$ can generally represent image content and data of multi-dimensional acquisitions.

For unknown coil sensitivities $c$, Equation (2.47) poses a linear inverse problem, where $\boldsymbol{y}$ is the measured variable and $\boldsymbol{x}$ is to be determined. Equation (2.47) can also be considered a nonlinear inverse problem if both $\boldsymbol{x}$ and $\boldsymbol{c}$ are treated as unknowns. This case will be covered in detail in Chapter 5 . In this section, we will focus on the linear formulation. 
In practical MRI experiments Equation (2.47) will not possess a solution, e.g. due to the inconsistencies introduced by noise. Thus, a solution can be defined as the vector $\boldsymbol{x}$, which minimizes the least-squares error $\|F \boldsymbol{x}-\boldsymbol{y}\|_{L^{2}}^{2}$. However, infinitely many vectors $\boldsymbol{x}$ will satisfy this condition if the kernel of $F$ consists of more elements than just the zero vector, $\operatorname{ker}(F) \supset\{\mathbf{0}\}$. Therefore, the solution is typically defined as the vector $\boldsymbol{x}^{\star}$ with minimum L2 norm $\left\|\boldsymbol{x}^{\star}\right\|_{L^{2}}^{2}$ which fulfills

$$
\boldsymbol{x}^{\star}=\underset{\boldsymbol{x}}{\operatorname{argmin}}\|F \boldsymbol{x}-\boldsymbol{y}\|_{L^{2}}^{2} .
$$

The direct formula for this $x^{\star}$ is given by the Moore-Penrose pseudo-inverse [99101],

$$
\boldsymbol{x}^{\star}=F^{\dagger} \boldsymbol{y}, \quad F^{\dagger}:=\lim _{\lambda \rightarrow 0}\left(F^{H} F+\lambda I\right)^{-1} F^{H},
$$

with identity matrix $I$.

REgULARIZATION AND COMPRESSED SENSING To allow for a numerically stable solution of badly conditioned linear systems, prior knowledge can be incorporated by introducing regularization terms in the cost function

$$
\boldsymbol{x}^{\star}=\underset{\boldsymbol{x}}{\operatorname{argmin}}\|F \boldsymbol{x}-\boldsymbol{y}\|_{L^{2}}^{2}+\sum_{i=1}^{N_{\mathrm{R}}} R_{i}(\boldsymbol{x}),
$$

with $N_{\mathrm{R}}$ the total number of regularization terms and $R_{i}(\boldsymbol{x})$ different convex penalty functions.

The simplest case made up of one quadratic penalty term $R(\boldsymbol{x})=\lambda\|\Gamma \boldsymbol{x}\|_{L^{2}}^{2}$ is called Tikhonov regularization, or to be more precise, Tikhonov-Phillips regularization [102],

$$
\boldsymbol{x}^{\star}=\underset{\boldsymbol{x}}{\operatorname{argmin}}\|\boldsymbol{F} \boldsymbol{x}-\boldsymbol{y}\|_{L^{2}}^{2}+\lambda\|\Gamma \boldsymbol{x}\|_{L^{2}}^{2}
$$

with $\Gamma$ a suitably chosen transform matrix. Equation (2.52) has the closed form solution ${ }^{3}$

$$
\boldsymbol{x}^{\star}=F_{\Gamma}^{\dagger} \boldsymbol{y}, \quad F_{\Gamma}^{\dagger}=\left(F^{H} F+\lambda \Gamma^{H} \Gamma\right)^{-1} F^{H} .
$$

Intuitively, Equation (2.52) seeks for a solution $x$ which provides a compromise between minimizing the data fidelity term $\|F \boldsymbol{x}-\boldsymbol{y}\|_{L^{2}}^{2}$ and keeping $\|\Gamma \boldsymbol{x}\|_{L^{2}}^{2}$ of reasonable size. The Lagrange parameter $\lambda$ tunes the influence of the penalty term and is generally chosen heuristically $[15,103]$.

Another popular approach for regularization is the use of the Li norm,

$$
\boldsymbol{x}^{\star}=\underset{\boldsymbol{x}}{\operatorname{argmin}}\|F \boldsymbol{x}-\boldsymbol{y}\|_{L^{2}}^{2}+\lambda\|\Gamma \boldsymbol{x}\|_{L^{1}} .
$$

3 Note that in the limit of $\lambda \rightarrow$ o Equation (2.53) recovers the Moore-Penrose pseudo-inverse Equation $(2.50)$. 
Compared to the L2 norm, which - for its quadratic nature - penalizes large values in particular, the Li norm penalizes small values much stronger. This promotes sparse solutions, especially when the information content of the image vector $\boldsymbol{x}$ can be localized in few non-vanishing components by a sparsifying transform $\Gamma$, while keeping the noise distributed over all pixels. The concept of sparsity had successfully been utilized for data compression [104] before its virtue for data acquisition was realized and the term compressed sensing was coined [14, 15, 105]. In fact, the concept of compressed sensing can be utilized to recover artifact-free images even for sub-Nyquist undersampling schemes. These undersampling schemes should provide sufficient incoherence to generate noise-like undersampling artifacts, which can then be removed in a sparse domain using the Li norm.

Two of the most popular sparsifying transforms in the field of MRI are the wavelet transform and the finite-differences transform. Medical images are known to be naturally sparse in the wavelet domain, for which the wavelet transform is mainly used to sparsify the spatial dimensions [15]. On the other hand, timeresolved medical image series often contain temporally piecewise constant regions with occasional rapid intensity variations. Therefore, the finite-differences transform $\Gamma:=D$, with

$$
\|D x\|_{L^{1}}:=\sum_{i=1}^{N_{t}-1}\left|x_{i+1}-x_{i}\right|
$$

can be applied to penalizes the sum of absolute variations over all frames $N_{t}$ in the temporal domain [15, 103]. Accordingly, Equation (2.55) is commonly referred to as the Total Variation (TV) of $\boldsymbol{x}$.

As opposed to Tikhonov-Phillips regularization, no general and convenient closed form solution for minimization problems with Li penalties exists. Moreover, solving inverse problems using a direct matrix inversion poses large demands on memory and computational power and suffers from limited flexibility as it does not allow for the use of arbitrary non-Cartesian sampling schemes. To overcome these limitations, matrix-free operator-based iterative methods can be utilized.

iterative optimization Algorithms Many successful iterative optimization algorithms have been proposed to solve regularized inverse problems of different kinds, e.g. [106-109]. A detailed discussion of the mathematical requirements and convergence properties of these methods is beyond the scope of this thesis. Instead, we will focus on the intuitive understanding of the two algorithms relevant for the experiments of this work: The Conjugate Gradient (CG) method and the Alternating Direction Method of Multipliers (ADMM).

The CG method developed by Hestenes and Stiefel [109] is used to solve linear inverse problems of type

$$
\boldsymbol{y}=A \boldsymbol{x}
$$


where $A$ is a symmetric, positive definite matrix. To fulfill this requirement, the Tikhonov-Phillips-regularized minimization problem of Equation (2.52) can be transformed into the equivalent Gauss normal equation

$$
\left(F^{H} F+\lambda \Gamma^{H} \Gamma\right) \boldsymbol{x}=F^{H} \boldsymbol{y} .
$$

The CG method was first introduced in the context of MRI image reconstruction by [110]. It is inspired by the gradient descent algorithm [103], but enforces the search directions $d_{i}$ to be $A$-orthogonal, i.e. o $=d_{j}^{T} A d_{i}$ for all $i, j$. It can be shown that the solution $x_{n}$ for iteration step $n$ is element of a Krylov subspace $K_{n}=$ $\boldsymbol{x}_{\boldsymbol{0}}+\operatorname{span}\left\{\boldsymbol{r}_{\mathrm{O}}, A \boldsymbol{r}_{\mathrm{O}}, \ldots, A^{n-1} \boldsymbol{r}_{\mathrm{O}}\right\}$ with $\boldsymbol{r}_{\mathrm{O}}$ the initial residual $\boldsymbol{r}_{\mathrm{O}}=\boldsymbol{y}-A \boldsymbol{x}_{\mathrm{o}}$. This $\boldsymbol{x}_{n}$ minimizes the distance $\left\|\boldsymbol{x}_{n}-\overline{\boldsymbol{x}}\right\|_{A}$ to the exact solution $\overline{\boldsymbol{x}}$ in the norm defined by $\|\boldsymbol{x}\|_{A}=\sqrt{\boldsymbol{x}^{H} A \boldsymbol{x}}$. The great benefit of this concept compared to the gradient decent approach is, that each descent direction is visited only once, which leads to faster convergence. More details on the algorithm can be found in [111].

The ADMM can be viewed as blend of augmented Lagrangian and dual decomposition methods. It was originally conceived in the 1970 S $[112,113]$ and extensively reviewed in [108]. Though not providing the fastest convergence rates, the ADMM is still extremely popular and widely used for its ability to cope with an arbitrary amount of convex penalty functions. This is achieved by splitting the minimization into smaller, local subproblems, which are then coordinated to find the actual solution to the global problem. The algorithm can be applied to problems of type

$$
\begin{array}{ll}
\text { minimize } & f(\boldsymbol{x})+g(\boldsymbol{z}) \\
\text { subject to } & A \boldsymbol{x}+B \boldsymbol{z}=\boldsymbol{q},
\end{array}
$$

where, simply put, $f$ and $g$ are convex functions, $\boldsymbol{x}, \boldsymbol{z}$ and $\boldsymbol{q}$ vectors and $A, B$ matrices. The corresponding augmented Lagrangian is given by

$$
L_{\rho}(\boldsymbol{x}, \boldsymbol{z}, \boldsymbol{v})=f(\boldsymbol{x})+g(\boldsymbol{z})+\boldsymbol{v}^{T}(A \boldsymbol{x}+B \boldsymbol{z}-\boldsymbol{q})+(\rho / 2)\|A \boldsymbol{x}+B \boldsymbol{z}-\boldsymbol{q}\|_{L^{2}}^{2},
$$

The ADMM minimizes $L_{\rho}$ in an alternating fashion with respect to either $\boldsymbol{x}$ or $\boldsymbol{z}$, while keeping the other variables fixed.

$$
\begin{aligned}
& \boldsymbol{x}_{n+1}:=\underset{\boldsymbol{x}}{\operatorname{argmin}} L_{\rho}\left(\boldsymbol{x}, \boldsymbol{z}_{n}, \boldsymbol{v}_{n}\right), \\
& \boldsymbol{z}_{n+1}:=\underset{\boldsymbol{z}}{\operatorname{argmin}} L_{\rho}\left(\boldsymbol{x}_{n+1}, \boldsymbol{z}, \boldsymbol{v}_{n}\right), \\
& \boldsymbol{v}_{n+1}:=\boldsymbol{v}_{n}+\rho\left(A \boldsymbol{x}_{n+1}+B \boldsymbol{z}_{n+1}-\boldsymbol{q}\right) .
\end{aligned}
$$

The update of $\boldsymbol{v}$ balances the individual solutions.

The subproblems for $\boldsymbol{x}$ and $\boldsymbol{z}$ are generally of simpler type and can be written in terms of proximity operators

$$
\operatorname{prox}_{R, \rho}(\overline{\boldsymbol{x}}):=\underset{\boldsymbol{x}}{\operatorname{argmin}}\left(\frac{\rho}{2}\|\boldsymbol{x}-\overline{\boldsymbol{x}}\|_{L^{2}}^{2}+R(\boldsymbol{x})\right),
$$


which represent generalized gradient descent operations and are applicable even for non-smooth cost functions. For common regularization penalties $R(\boldsymbol{x})$ the proximity operator is available in a computationally efficient form. ${ }^{4}$

To draw the connection to the minimization problem stated in Equation (2.54), the functions and variables of Equation (2.60) can be identified as follows:

$$
\begin{gathered}
f(\boldsymbol{x}):=\|F \boldsymbol{x}-\boldsymbol{y}\|_{L^{2}}^{2} \\
g(\boldsymbol{z}):=\lambda\|\boldsymbol{z}\|_{L^{1}} \\
A:=\Gamma, B:=-1, \boldsymbol{q}:=\mathrm{O} \Rightarrow \boldsymbol{z}=\Gamma \boldsymbol{x} .
\end{gathered}
$$

\subsubsection{Non-Cartesian Image Reconstruction}

The Fourier transform is an essential part of the forward operator introduced in Equation (2.48) of the previous section. Unfortunately, the Discrete Fourier Transform (DFT) for discretely sampled data is a computationally demanding procedure, which requires $O\left(N_{\text {data }}^{2}\right)$ operations, where $N_{\text {data }}$ is the total number of samples, including all spatial and temporal dimensions as well as all coils. Luckily, the computational burden can be significantly reduced by the use of the FFT, which only requires $O\left(N_{\text {data }} \log N_{\text {data }}\right)$ operations [116]. Without the FFT, efficient image reconstruction would not be feasible even with the latest high-performance computers - particularly as iterative optimization strategies rely on the repeated execution of the forward operator and its adjoint.

However, one major limitation of the FFT is the fact that it can only process Cartesian data. Hence, a straight forward application to non-Cartesian imaging is not possible and demands particular attention. Therefore, the Non-Uniform Fast Fourier Transform (NUFFT) $\mathcal{G}$ was conceived [117]. For the sake of comprehensibility, we will first introduce the steps involved in the adjoint NUFFT $\mathcal{G}^{H}$, which describes the transformation of non-Cartesian k-space data onto a rectilinear grid in image domain. Essentially, the adjoint NUFFT combines a k-space interpolation with a conventional FFT-based reconstruction.

The interpolation of non-Cartesian data onto a rectilinear grid is not trivial and provides several pitfalls, which can degrade the outcome, since any modification of the original data can manifest disturbing modulation effects in the corresponding Fourier domain. We follow $[49,118,119]$ for a brief description of the procedure and use a continuous formulation for the sake of comprehensibility.

4 The solution to proximity operators with L2 norm penalty of type $R(\boldsymbol{x})=\|M \boldsymbol{x}-\boldsymbol{b}\|_{L^{2}}^{2}$ with constant vector $\boldsymbol{b}$ is given in closed form if $M$ is a unitary operator and can be calculated using the CG algorithm if $M$ is non-unitary. The solution to proximity operators with L1 norm penalties of type $R(\boldsymbol{x})=\|M \boldsymbol{x}-\boldsymbol{b}\|_{L^{1}}$ can be obtained by soft-thresholding if $M$ is unitary. If $M$ is non-unitary a solution can be determined iteratively, e.g. using FISTA [108, 114, 115]. 
In a measurement process, the continuous signal $S(\boldsymbol{k})$ is sampled at $N_{\text {tot }}$ discrete non-Cartesian locations $\boldsymbol{k}_{i}$. This sampling can be understood as the multiplication of the continuous signal $S(\boldsymbol{k})$ with a sum of Dirac-delta functions,

$$
\zeta S(\boldsymbol{k}):=\sum_{i=1}^{N_{\text {tot }}} \delta\left(\boldsymbol{k}-\boldsymbol{k}_{i}\right) S(\boldsymbol{k}) .
$$

This signal is then convolved with a convolution kernel $\mathcal{K}$, which yields the interpolated signal

$$
S_{\text {interp }}(\boldsymbol{k})=\int_{K} \mathcal{K}(|\boldsymbol{k}-\boldsymbol{\kappa}|) \cdot \zeta S(\boldsymbol{\kappa}) \mathrm{d} \boldsymbol{\kappa},
$$

where the integral covers the entire k-space $K$. The preferred convolution kernel $\mathcal{K}$ would be the sinc function, since the related convolution corresponds to a multiplication of the compact image space signal with a rect function. Thus, signal distortion would be avoided [88, 117]. However, the sinc function as convolution kernel is infeasible due to its infinite support. Therefore, the Kaiser-Bessel kernel was proposed in [120] as a compact substitute. Next, the convolved signal is sampled onto a rectilinear grid. This sampling can be modeled by the multiplication of a comb-function $\mathbb{I}_{\Delta k}$ consisting of Dirac-delta functions on a Cartesian grid with sampling distance $\Delta k$. Subsequently, the inverse FFT $\mathcal{F}_{\mathrm{FFT}}^{-1}$ can be performed. Finally, a roll-off correction is applied by multiplying the inverse Fourier transform $\tilde{\mathcal{K}}^{-1}$ of the kernel, where the tilde denotes Fourier transformed quantities.

In operator notation, the adjoint NUFFT can be expressed by ${ }^{5}$

$$
\begin{aligned}
\mathcal{G}^{H} \zeta S(\boldsymbol{k}) & =\tilde{\mathcal{K}}^{-1} \mathcal{F}_{\mathrm{FFT}}^{-1} \mathbb{I}_{\Delta k} \mathcal{K} * \zeta S(\boldsymbol{k}) \\
& =\tilde{\mathcal{K}}^{-1} \tilde{\mathbb{I}}_{\mathrm{FOV}} * \tilde{\mathcal{K}} \tilde{\zeta} * \tilde{S}(\boldsymbol{x}),
\end{aligned}
$$

where $\tilde{S}(\boldsymbol{x})$ denotes the ideal continuous object in image space. Here, the Fourier convolution theorem was used to obtain the second line, which provides insights into the effects of the individual operations, illustrated in Figure 2.7. The sampling of the continuous signal $S(\boldsymbol{k})$ corresponds to a convolution of the ideal and continuous object function $\tilde{S}(\boldsymbol{x})$ (Figure 2.7a) with the Fourier transformed sampling operator $\tilde{\zeta}$. This effects blurring and furthermore creates pattern-specific side-lobes, which can extend outside of the actual field of view (Figure 2.7b). The convolution by the finite interpolation kernel $\mathcal{K}$ translates into a multiplication with $\tilde{\mathcal{K}}$ in the Fourier domain, which apodizes the signal (Figure 2.7c) and which is compensated by the roll-off correction. The multiplication of the comb function $\mathbb{I I}_{\Delta k}$ translates into a convolution with $\tilde{\mathbb{I}}_{\mathrm{FOV}}$. This causes a repetition of $\tilde{\mathcal{K}} \tilde{\zeta} * \tilde{S}(\boldsymbol{x})$ in the image domain, with replicates located $1 / \Delta k=$ FOV apart (Figure $2.7 \mathrm{~d}$ ).

To avoid aliasing through the leakage of side-lobes into the actual object, the sampling is usually performed on a two-fold oversampled grid $\mathbb{I}_{\Delta k / 2}$, yielding

5 Note that with the notation of Equation (2.47) the signal function $\zeta S(\boldsymbol{k})$ is a different representation of the sampled signal $\boldsymbol{y}$. 


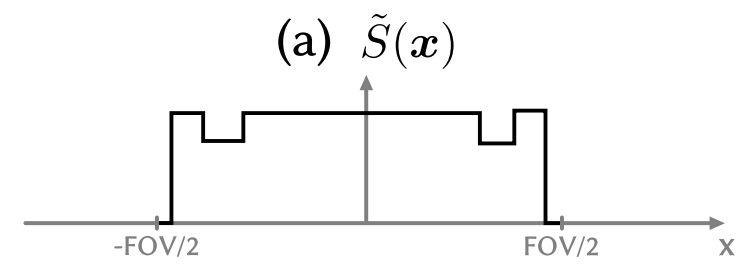

(b) $\tilde{\zeta} * \tilde{S}(\boldsymbol{x})$

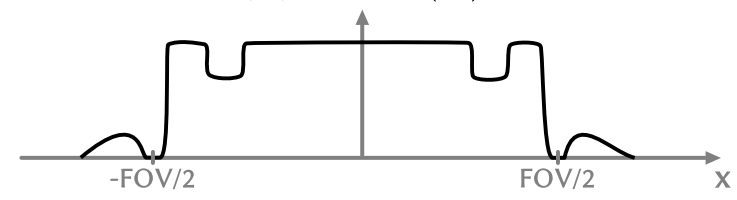

(c) $\tilde{\mathcal{K}} \tilde{\zeta} * \tilde{S}(\boldsymbol{x})$

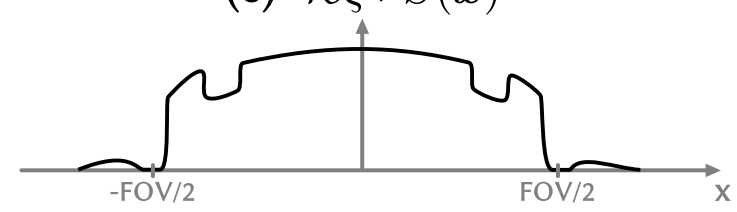

(d) $\tilde{\mathbb{I}}_{\mathrm{FOV}} * \tilde{\mathcal{K}} \tilde{\zeta} * \tilde{S}(\boldsymbol{x})$

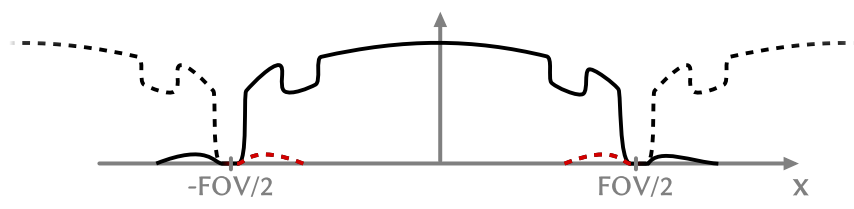

(e) $\tilde{\mathbb{I I}}_{2 \mathrm{FOV}} * \tilde{\mathcal{K}} \tilde{\zeta} * \tilde{S}(\boldsymbol{x})$

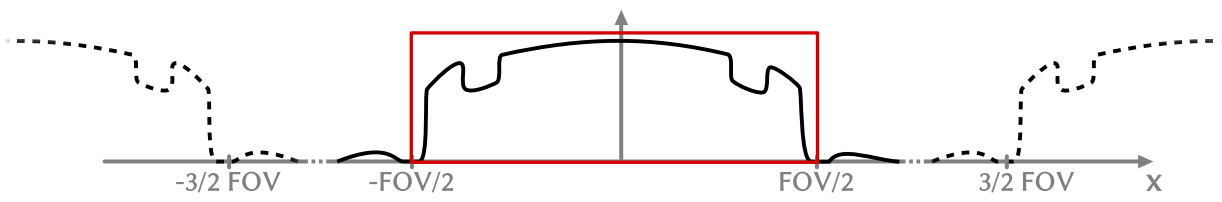

Figure 2.7.: Illustration of modulation effects related to the adjoint NUFFT. In image domain, the continuous object signal $\tilde{S}(\boldsymbol{x})$ (a) is convolved with the Fourier transformed sampling function $\tilde{\zeta}$, which induces blurring and side-lobes (b). The multiplication of the Fourier transformed convolution kernel $\tilde{\mathcal{K}}$ apodizes the object $(\mathrm{c})$. The subsequent convolution with the sampling function $\tilde{\mathbb{I}}_{\mathrm{FOV}}$ would create replicates from which side-lobes (highlighted in red) leak into the actual object (d). Therefore, sampling is performed onto a two-fold oversampled grid to shift the replicates further apart, which allows the object to be extracted by cropping (red rectangle) (e). The graphic was inspired by [119]. 
the reduced sampling distance $\Delta k / 2$. Consequently, the FOV in image domain is increased by a factor of two. Thus, the aliasing of side-lobes into the replicates created by convolution with $\tilde{\mathbb{I}}_{2 \mathrm{FOV}}$ are shifted further away from the object and can be eliminated by cropping the doubled FOV to its original size (Figure 2.7e).

The actual NUFFT $\mathcal{G}$ essentially conducts the described procedure in reverse order and with adjoint operators to map a Cartesian image $\mathbb{I}_{\Delta x} \tilde{S}(\boldsymbol{x})$ onto nonCartesian k-space locations: 6

$$
\mathcal{G} \mathbb{I}_{\Delta x} \tilde{S}(\boldsymbol{x})=\zeta \mathcal{K} * \mathcal{F}_{\mathrm{FFT}} \tilde{\mathbb{I}}_{\mathrm{FOV}} * \tilde{\mathcal{K}} \mathbb{I}_{\Delta x} \tilde{S}(\boldsymbol{x})
$$

In the inverse problem formulation of MRI image reconstruction, the forward operator for non-Cartesian imaging $F_{\mathcal{G}}$ can easily be adapted from the original definition $F$ (Equation (2.48)) by replacing the Fourier transform $\mathcal{F}$ and the projection operator $P$ with the NUFFT operator $\mathcal{G}$,

$$
F_{\mathcal{G}}:=\mathcal{G C}
$$

For non-Cartesian imaging, iterative optimization of Equation (2.49) requires the repeated application of the normal operator $F_{\mathcal{G}}^{H} F_{\mathcal{G}}$, which essentially concatenates the NUFFT and its adjoint. Such a composition corresponds to a convolution with a point-spread function, which can be realized by two FFTs and a multiplication [121]. Like this, the interpolation step can be separated from the iterative optimization, which enables efficient computation [41].

Note that unlike the Cartesian case, where the appropriately scaled adjoint FFT is likewise the inverse FFT, the NUFFT is not unitary due to the involved convolution step. Hence, the adjoint of the NUFFT is not its inverse.

One way to invert the NUFFT operation and thus to obtain an image $\boldsymbol{x}$ from non-Cartesian data $\boldsymbol{y}$ is to solve the inverse problem Equation (2.49) with forward operator Equation (2.67). Alternatively, the gridding approach can be employed, which approximates the inverse NUFFT by combining a density compensation of the sampled k-space data with an adjoint NUFFT. The necessity of the density compensation originates from the fact that non-Cartesian data are by definition not sampled equidistantly, which must be accounted for to avoid image blurring. Typically, the density compensation function is estimated using a Voronoi diagram [122]. However, for radial imaging density compensation can be performed using the Ram-Lak filter $[123,124]$ which makes up for the higher density of the k-space center compared to the peripheral regions.

6 Note that with the notation of Equation (2.47) the image function $\mathbb{I}_{\Delta x} \tilde{S}(\boldsymbol{x})$ is a different representation of the image $\boldsymbol{x}$ on discrete Cartesian samples. 


\subsubsection{Gradient Delay Correction}

Besides all benefits, non-Cartesian imaging does not only complicate the image reconstruction, but also poses challenges in the measurement process. So far we have considered undistorted signal generation and data acquisition, neglecting the influence of system imperfections and hardware limitations. Indeed, over the years MRI vendors have improved the quality and standards of their systems considerably, but even the best maintained and calibrated scanners cannot guarantee ideal conditions. Particularly, the use of fast switching high-amplitude gradients as in FLASH and bSSFP sequences can cause violations of the idealized assumptions underlying MR image formation. In this section, we will only focus on hardwaretiming errors and eddy currents, which - if not properly accounted for - can distinctly corrupt the resulting image quality, particularly for radial imaging. A good overview of other hardware and non-hardware related MRI artifacts can be found in $[81,125]$.

GRADIENT DELAY THEORY Faraday's and Lenz's law state that any change of the magnetic flux induces a current in an electric circuit, which creates a magnetic field that counteracts the change of the magnetic flux. In the context of MRI, these eddy currents are created by ramping magnetic field gradients up and down. The $z$ component $B_{z}^{\mathrm{ec}}$ of the magnetic field strength created by eddy currents is spatially dependent and can be approximated by a Taylor expansion [81]

$$
B_{z}^{\mathrm{ec}}(\boldsymbol{r}, t)=b_{\mathrm{o}}(t)+\boldsymbol{r} \cdot \boldsymbol{g}(t)+\ldots
$$

The $o^{\text {th }}$ order term is often referred to as $B_{0}$ eddy current, whereas the $1^{\text {th }}$ order term is called the linear eddy current. Higher order terms can generally be neglected [81]. The vector $\boldsymbol{g}(t)$ represents eddy-current-induced magnetic field gradients along all three spatial axis.

The magnetic field defined in Equation (2.68) can be interpreted as eddy-currentinduced field homogeneities, which lead to a spatio-temporally dependent phase accumulation during signal acquisition [126],

$$
\Phi^{\mathrm{ec}}(\boldsymbol{r}, t)=\gamma \int_{\mathrm{o}}^{t} B_{z}^{\mathrm{ec}}(\boldsymbol{r}, \tau) d \tau \approx \gamma \int_{\mathrm{o}}^{t} b_{\mathrm{o}}(\tau) d \tau+\int_{\mathrm{o}}^{t} \boldsymbol{r} \cdot \boldsymbol{g}(\tau) d \tau .
$$

Note that for each acquired line in k-space the strength of the applied gradients may differ, see Equation (2.39), and consequently the corresponding values for $b_{0}(t)$ and $\boldsymbol{g}(t)$ may vary, too.

The $B_{0}$ eddy current contributes a global phase for each acquired k-space line, while the first-order eddy current causes a spatial linear phase-variation in image space, which corresponds to a shift in k-space. The $B_{0}$ eddy current compensation is mainly required for small-bore high-field MRI systems, which are usually 
employed for animal studies [126]. In contrast, linear eddy currents cause sever artifacts on most scanners if not properly accounted for. We therefore consider the effects of linear eddy currents in more detail.

The individual components of $\boldsymbol{g}(t)$ can be modeled by [127, 128]

$$
g_{i}(t)=\sum_{j=x, y, z}-\left(\frac{d}{d t} G_{j}(t)\right) * E_{i j}(t) \approx-\left(\frac{d}{d t} G_{i}(t)\right) * E_{i i}(t), \quad i=x, y, z,
$$

where $G_{i}(t)$ is the $\mathrm{i}^{\text {th }}$ component of an applied magnetic field gradient, $*$ denotes a convolution and $E(t)$ is the eddy current impulse response. In Equation (2.70) the cross-terms with $i \neq j$ can usually be neglected. Note that the eddy currents are stronger for gradients with steep slopes $d G_{i} / d t$, which are commonly used for fast imaging sequences such as bSSFP and FLASH.

It was shown in $[127,128]$ that the eddy current impulse response can accurately be represented by a superposition of few decaying multi-exponential functions

$$
E(t)= \begin{cases}\sum_{n} \alpha_{n} e^{-t / \tau_{n}} & t \geq 0 \\ 0 & t<0\end{cases}
$$

of amplitude $\alpha_{n}$ and time-constant $\tau_{n}$.

To gain an intuitive understanding of the effect of eddy currents, we consider a trapezoidal gradient with maximum strength $G_{i}^{\max }$, ramp time $T_{\text {ramp }}$ and corresponding slope [81]

$$
\frac{d}{d t} G_{i}(t)=\frac{G_{i}^{\max }}{T_{\text {ramp }}}, \quad 0 \leq t \leq T_{\text {ramp }}
$$

where $t=0$ signifies the beginning of the ramp. For $n=1$ and in combination with Equations (2.70) and (2.71), we obtain

$$
g_{i}(t)=-\frac{G_{i}^{\max }}{T_{\mathrm{ramp}}} \alpha \tau\left(1-e^{-t / \tau}\right), \quad 0 \leq t \leq T_{\mathrm{ramp}}
$$

For large time-constants $\tau$ we can approximate $e^{-t / \tau} \approx 1-t / \tau$, which yields

$$
g_{i}\left(t=T_{\text {ramp }}\right) \approx-G_{i}^{\max } \alpha .
$$

These kind of eddy current induced magnetic field gradients are - in first order approximation - time-independent and possess an opposed sign for rising and falling ramps. Therefore, they can be assumed to partially cancel out one another, particularly if the flat-top time of the gradient $G_{i}(t)$ is smaller than the time-constant $\tau$.

For small values of $\tau$ we find

$$
g_{i}(t) \approx-\frac{G_{i}^{\max } \alpha \tau}{T_{\text {ramp }}}
$$


which yields the net magnetic field gradient

$$
G_{i}^{\text {net }}(t)=G_{i}(t)+g_{i}(t) \approx \frac{G_{i}^{\max } \cdot(t-\alpha \tau)}{T_{\text {ramp }}}=G_{i}(t-\alpha \tau), \quad 0 \leq t \leq T_{\text {ramp }}
$$

Thus, short linear eddy currents delay the start of an applied gradient waveform on axis $i$ by the amount $\alpha \tau$. This finding is the foundation for many gradient delay correction strategies $[50,81,85,129]$ and also constitutes the basis for our study in Chapter 3 .

Additionally to the delays caused by eddy currents, other hardware related effects such as group delays in the amplifier or in the transmit and receive electronics can deviate the start of magnetic field gradients [81]. In fact, delays from eddy currents with short time-constants cannot be distinguished from actual system delays, for which gradient delays are sometimes referred to as apparent system delays [85].

Various approaches to account for gradient delays using preemphasis hardware exist and are implemented in current scanner hardware [81, 127, 130-132]. However, pre-compensation capabilities are limited by the gradient amplifier bandwidth [81] for which gradient delays cannot entirely be eliminated.

EFFECT OF GRADIENT DELAYS ON CARTESIAN AND RADIAL IMAGING For Cartesian k-space trajectories, sampled line-by-line in a consistent read-out direction, gradient delays do not impact the resulting image quality. If all lines in kspace are shifted by the same amount and in the same direction, the magnitude image of the reconstruction remains uncorrupted and the shift in k-space is, in accordance with the Fourier shift theorem, translated into a linear phase in image space. In radial imaging gradient delays induce inconsistent shifts for all acquired lines due to the varying projection angle. As a result, both the magnitude and the phase image are affected and artifacts become visible. Figure 2.8 exemplifies this behavior on a numerical simulation of a Shepp-Logan phantom.

\subsubsection{Conclusion}

While the use of parallel imaging is nowadays the clinical standard to accelerate scans, the inverse problem formulation in combination with iterative reconstruction algorithms in general, and compressed sensing in particular, is a relatively new trend, which exhibits great potential for further scan time reduction.

The processing of data, which are not sampled on a rectilinear grid can naturally be integrated into the forward operator by means of the NUFFT. However, non-Cartesian imaging is prone to system imperfections. Gradient delays are particularly problematic for fast switching sequences and cause image degradation in radial imaging. Only with the gradient delays properly accounted for, a high quality image reconstruction is feasible. 
(a) Cartesian

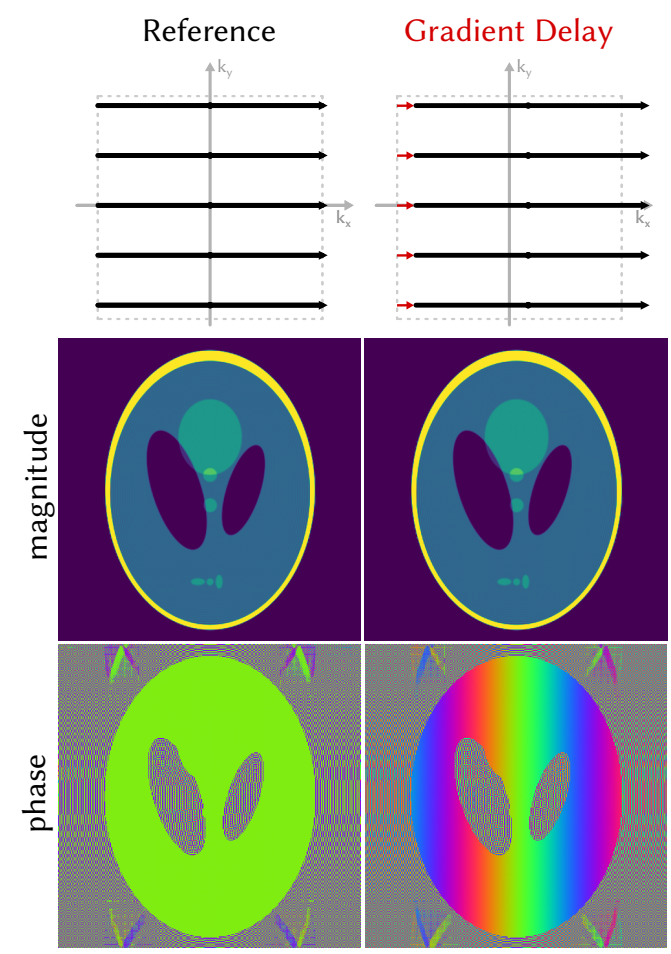

(b) Radial

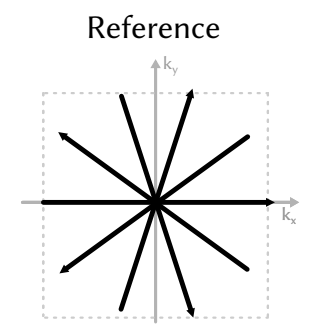

Gradient Delay
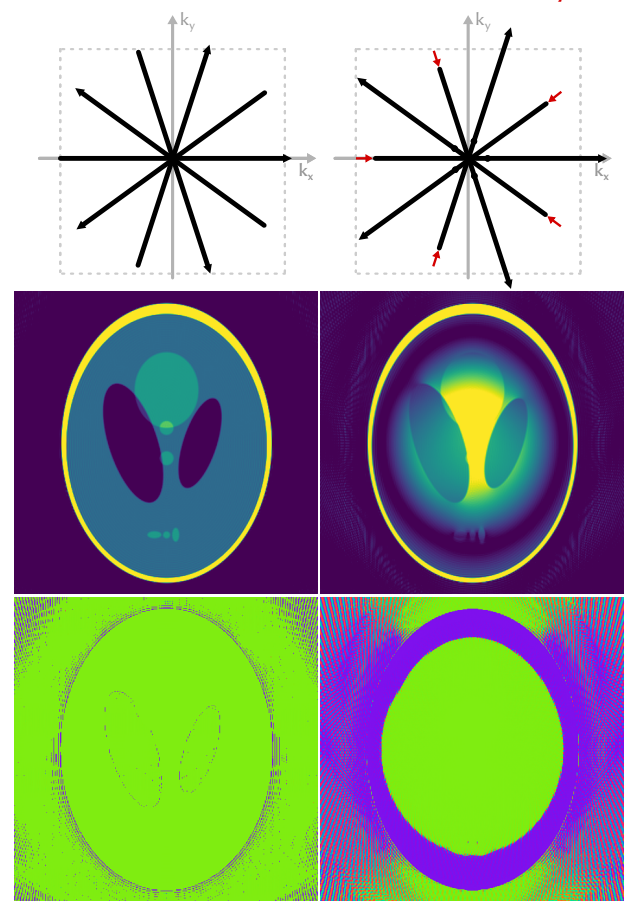

Figure 2.8.: Schematic and numerical simulation to demonstrate the effect of gradient delays for Cartesian (a) and radial (b) k-space trajectories. The top row shows schematic reference k-spaces for Cartesian and radial sampling, as well as their shifted counterparts. The shift direction is highlighted by red arrows. Underneath, reference images and images affected by gradient delays are depicted for Cartesian and radial trajectories. For Cartesian line-by-line sampling with consistent read-out direction, gradient delays and their corresponding $\mathrm{k}$-space shifts are translated into a linear phase in image space and do not corrupt the magnitude image. For radial imaging, the lines are shifted in different directions, which causes artifacts in the magnitude image.

To account for gradient delay effects, we will introduce in Chapter 3 a novel and intuitive method to estimate the gradient delays for radial imaging and compare it to the (AC-)Adaptive method [133, 134], which is described in the Appendix A. In Chapter 5 we will introduce the Iteratively Regularized Gauss-Newton Method (IRGNM) algorithm, which solves the nonlinear inverse problems by local linearization. The linearized cost function is then minimized via the CG method. We will use this approach to reconstruct severely undersampled SMS acquisitions and to determine coil sensitivity maps. We will make use of the ADMM in Chapter 7 to solve linear inverse problems with combined in-plane wavelet and (spatio-) temporal TV regularization. This enables the reconstruction of highly undersampled multi-dimensional cardiac MRI acquisitions. 


\subsection{CONVENTIONAL METHODS IN CARDIAC MRI}

Despite of all progress in data acquisition and image reconstruction, MRI always has been and still must be considered a slow imaging modality. Consequently, any kind of motion can lead to artifacts and image degradation. Due to the ubiquitous beating heart and patient respiration, cardiac MRI faces a huge challenge. In this section, we will introduce the ECG as a means to detect and section the cardiac motion and present several approaches used in clinical practice to deal with respiration.

\subsubsection{Cardiac Gating using the Electrocardiogram}

The ECG is a monitoring device, which uses electrodes to image the heart's electrical activity. In particular, it detects the QRS complex, i.e. the most characteristic wave in the electric signal, which is caused by the depolarization of the ventricles. The QRS complex does not define the actual start of a cardiac cycle, but constitutes the most stable reference point to detect. The ECG allows for a segmented data acquisition of one or more phases of the cardiac cycle over multiple heartbeats [16]. Assuming a periodic heartbeat, this so called gated imaging can be used to generate results with a high spatial and temporal resolution.

In the context of cardiac MRI, the ECG was used from early on [17, 18]. In principle, two different gating strategies can be distinguished. For prospective gating, also know as cardiac triggering, data acquisition begins only after the detection of the QRS event and a user defined time delay. It is utilized to generate a static image of a particular phase of the cardiac cycle. For retrospective gating, data is continuously acquired. The simultaneously recorded ECG signal can be used to reorder or bin the data into the respective cardiac phases. Retrospective gating is used for CINE MRI, where the cardiac motion is temporally resolved. ECG-based cardiac gating is illustrated in Figure 2.9a and Figure 2.9b.

Since the ECG relies on the accurate detection of electric potential differences in the myocardium, its acquisition in the presence of a huge external magnetic field, varying magnetic field gradients and repeatedly applied RF pulses is a highly nontrivial task. While the bandwidth of RF pulses in MRI lies mainly in the megahertz range and can therefore be filtered out [135], the switching of the gradient fields happens in the kilohertz-range, which causes an apparent deterioration of the ECG signal, particularly for fast switching sequences [22]. Another impact on the ECG signal is induced by the interaction of the static external magnetic field and electrically charged particles and cells in the blood flow. Those cells experience a force perpendicular to their velocity. This phenomenon, know as magneto-hydrodynamic effect or Hall effect, can induce additional disturbing electrical fields within blood vessels $[23,24]$. 


\section{(a) Gating Strategies}

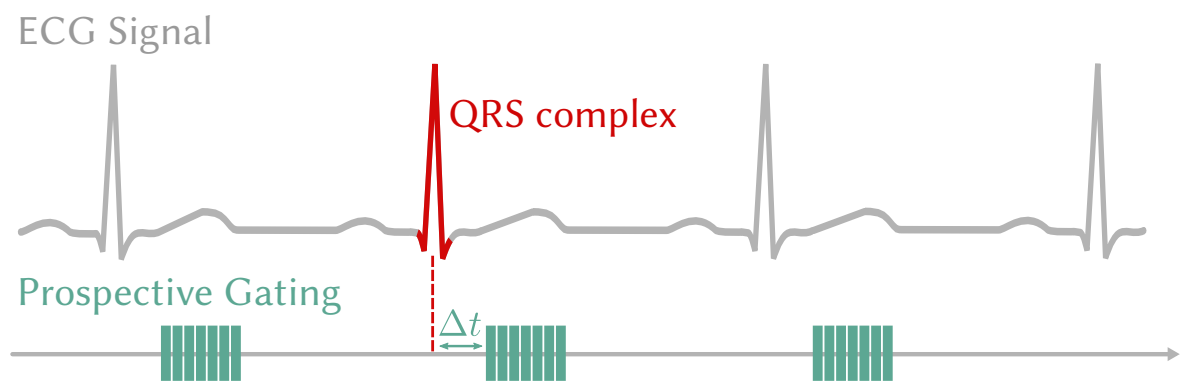

Retrospective Gating

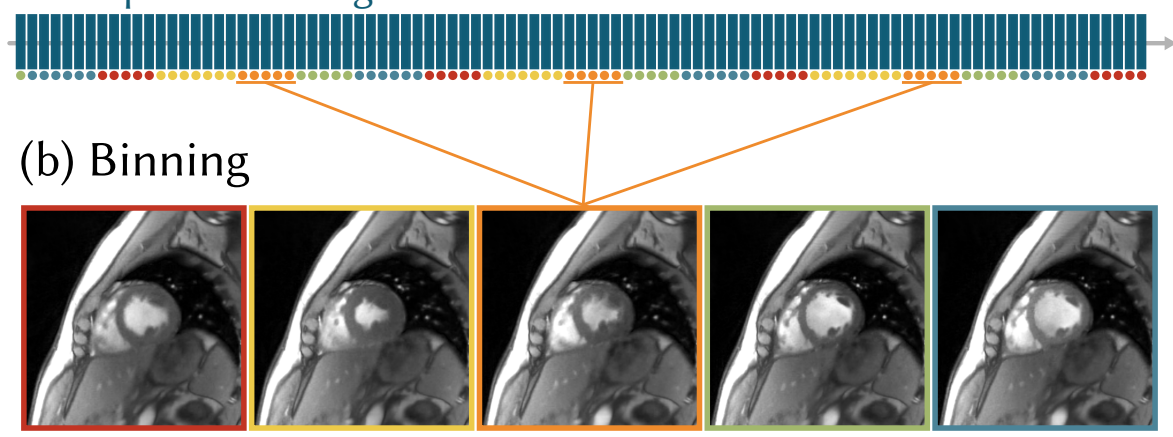

(c) Example

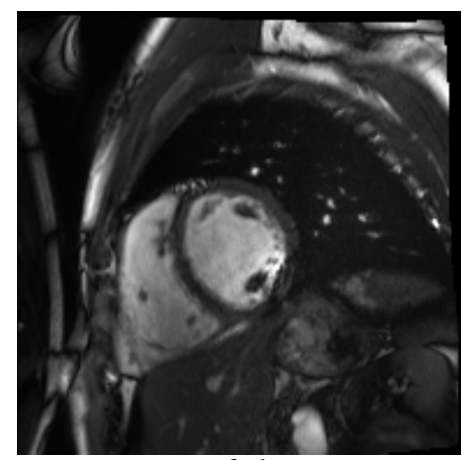

successful gating

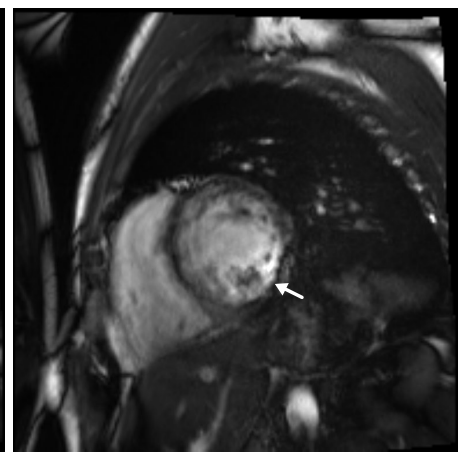

failed gating

Figure 2.9.: Illustration of cardiac gating using the ECG. (a) schematically shows the time-course of an ECG with one QRS complex highlighted in red. Furthermore, the data acquisition period for prospective and retrospective gating is indicated. For prospective gating, data acquisition of a specific cardiac phase is performed after each QRS complex, followed by a user-defined time delay $\Delta t$. For retrospective gating, data is continuously acquired. According to the ECG signal, each data block is then binned into its respective cardiac phase and data from various heartbeats are combined for image reconstruction of the cardiac cycle (b). In (c), an example for successful and failed cardiac ECG gating is depicted. 
The mentioned effects can induce ECG signal distortions and significantly impact the accuracy and precision of cardiac gating, which then results in artifacts through inaccurate binning, as Figure 2.9c shows. The impact of the MRI environment on the ECG signal is in general more severe the higher the field strength [25]. A seldomly used alternative to circumvent this problem is the peripheral pulse trigger [136]. However, the relation between cardiac cycle and peripheral pulse, which is usually detected using a device placed on the patients finger, is imprecise as it depends on the stroke volume and the arterial wall stiffness [137].

For more information on the use of ECG in the context of MRI, we refer the reader to the review article [138].

\subsubsection{Respiratory Monitoring and Breath-hold Commands}

Not only the beating heart but also respiration can induce motion related artifacts if not properly accounted for. Human respiration is a complex process which involves a combination of deformation as well as longitudinal and transverse translation of organs $[48,139]$. In fact, proper handling of respiratory motion constituted one of the major challenges in the early days of cardiac MRI and still is a topic of active research [11, 140-143]

The most simplistic but still most popular approach to deal with respiratory motion is to command breath-holds during acquisition. To obtain high-resolution images with sufficient spatial coverage, a single breath-hold usually does not suffice for gated acquisitions, thus the volunteer is repeatedly dictated to hold his breath after expiration for a period of about 8-15 seconds. This strategy can eliminate abdominal motion to a large extend, such that complementary data from various breath-holds can be acquired and combined. Nevertheless, there is no guarantee for a consistent heart position in each acquisition period, since the depth of breathing and corresponding breath-holds can be subject to variations. Further obvious drawbacks of commanding breath-holds are patient discomfort, the delay required for the patient to recover from a breath-hold and the need for patient compliance, which is especially problematic for sick patients and children.

Alternatively, respiratory belts or cushions can be utilized to monitor the breathing motion [144]. Such devices are commonly placed on or around the abdomen and are connected to a pneumatic pressure transducer, which relates pressure changes with circumferential variations of the abdomen caused by respiration [145]. Thereby, the patient's breathing motion can be tracked in real-time and dataacquisition can be restricted to a fixed respiratory state - mostly end-expiration - while the patient is allowed to breath freely. This, however, introduces a deadtime where no data is acquired. Despite the improved patient comfort, respiratory monitoring using external devices did not find widespread application in clinical environments, mainly due to its limited reliability [146, 147]. 
Finally, respiratory monitoring without the use of external devices can be achieved using Navigator Echoes (NAV) [21]. Here, the actual data acquisition is interleaved with additional slice-selective RF pulses used to generate projections through the abdomen. These projections are typically positioned to image the superior-inferior motion of the right hemi-diaphragm, i.e. the interface between liver and lung, and thus serve as scouts to track the respiratory motion. Similar to the respiratory belt, patients are allowed to breath freely as respiratory motion can be eliminated in the acquisition by confining data acceptance to e.g. end-expiration. The main drawback of the NAV approach is the prolonged measurement time due to the interleaved RF pulses. Moreover, these interleaves can disrupt the steady-state condition in certain sequences.

\subsubsection{Whole Heart Coverage}

For a thorough cardiac assessment, physicians require information about the entire heart. The common strategy to fulfill this requirement is the use of gated multi-slice MRI, where multiple slices are acquired in an interleaved or sequential fashion using a conventional gated single-slice cardiac MRI technique. Although being a robust and probably the most obvious way to cover the third spatial dimension, the conventional multi-slice approach has three major limitations. First, it is slow. Despite evident similarities between adjacent slices no mutual information is shared, e.g. through regularization. Hence, the acquisition-time increases proportionally to the number of acquired slices. Second, it introduces temporal inconsistencies. Since each slice is scanned individually, the results can only be synchronized retrospectively, which might impair the integrity of the imaged volume. Third, several stacks of slices with varying slice orientations, such as the short-axis or the fourchamber view, need to be acquired. This procedure is not only lengthy but also requires a trained technician for adequate slice planning.

\subsubsection{Conclusion}

Current approaches to deal with cardiac and respiratory motion can provide a high-quality output, but include a cumbersome placement of external devices, demand patient compliance and involve laborious slice-by-slice scanning. Instead, a fast and universal approach, which provides maximal patient comfort and which is applicable not only for compliant adults but also sick patients or children is desired. Promising modern approaches, that try to achieve this goal by enhanced data processing and the use of temporal and spatial correlations, will be covered in the subsequent section.

In Chapter 7 we will perform experiments employing the ECG, breath-holds and the respiratory belt for comparison and validation purposes. 


\subsection{ADVANCED METHODS FOR MULTI-DIMENSIONAL CARDIAC MRI}

The techniques and strategies described in the previous section still are the common choice for cardiac MRI assessments in the vast majority of hospitals worldwide - despite the apparent drawbacks and the huge potential for improvements.

In this section, we will introduce alternative approaches, which can overcome many of the aforementioned limitations. In particular, we will present concepts, which take advantage of multi-spatial and multi-temporal correlations to diminish the data demand with little sacrifice to image quality. Moreover, we will expound the idea of self-gated cardiac MRI to eliminate the dependency on external hardware or patient compliance and frame it in the context of dynamical systems theory and time-delayed embedding.

\subsubsection{Approaches Towards the Third Spatial Dimension}

For a thorough cardiac assessment single-slice information is insufficient and the use of conventional multi-slice imaging is lengthy, potentially introduces spatiotemporal inconsistencies and neglects the opportunity to exploit three-dimensional spatial correlations. Therefore, we present the alternative concepts of SMS and volumetric imaging to take advantage of the three dimensional nature of the problem.

Simultaneous multi-SLice IMAging Instead of measuring all desired slices separately, in SMS imaging multiple slices are excited and acquired simultaneously [148]. This does not only improve the SNR, but also guarantees inter-slice timeconsistency and allows for significantly reduced scan times [149, 150]. The acquired signal in SMS MRI is a superposition of the signals emitted by the individual slices. To facilitate the slice-disentanglement, a unitary slice-encoding scheme is commonly utilized. For this purpose, a possible option is Hadamard encoding [151], which - however - provides some restrictions regarding the choice of number of slices. The more popular - and likewise more natural - choice in the context of MRI is Fourier encoding.

To acquire and reconstruct $M$ slices we have to perform $M$ Fourier-encoded SMS measurements

$$
\tilde{\boldsymbol{y}}_{p}=\sum_{q=1}^{M} \Xi_{p q} \boldsymbol{y}_{q},
$$

with $\tilde{\boldsymbol{y}}_{p}$ the superposed signal for one encoding, which we will refer to as the signal of partition $p$. Here, the tilde symbol $\sim$ denotes Fourier-encoded variables. The signal of slice $q$ is represented by $\boldsymbol{y}_{q}$ and

$$
\Xi_{p q}=\exp \left(\frac{-2 \pi i(p-1)(q-1)}{M}\right)
$$


is the discrete $M \times M$ Fourier matrix.

The decoding of signal Equation (2.77) for fully sampled SMS acquisitions can be performed using the inverse Fourier matrix $\Xi_{q p}^{-1}$, which reveals the averaging nature of SMS MRI

$$
\boldsymbol{y}_{q}^{\mathrm{SMS}}:=\sum_{p=1}^{M} \Xi_{q p}^{-1} \tilde{\boldsymbol{y}}_{p} \stackrel{\text { Eq. }}{=} \sum_{p=1}^{(2.77)} \Xi_{q p}^{M} \sum_{m=1}^{M} \Xi_{p m} \boldsymbol{y}_{m}=\frac{\sum_{i=1}^{M} \boldsymbol{y}_{q}}{M}
$$

Since the averaging of signals diminishes the impact of noise, SMS acquisitions yield an SNR benefit of $\sqrt{M}$ compared to individual single-slice measurements [63].

However, the application of the inverse Fourier matrix to obtain disentangled slices is only possible for aligned trajectories, where the sampling positions are the same for all encodings $\tilde{\boldsymbol{y}}_{p}$. If a different, so-called interleaved trajectory is chosen for each encoding, the slices can no longer be disentangled by a simple mathematical operation and image reconstruction must be performed jointly for all slices. What sounds like an adverse complication at first eventually turns out to be highly advantageous. For interleaved trajectories, complementary k-space information for all slices is acquired in each encoding, which vastly augments the global k-space coverage. This also explains why the scan-time can be significantly reduced compared to conventional multi-slice measurements: The complementary k-space information from each encoding allows for a high undersampling of the individual encodings, while still preserving a good k-space coverage. This approach is also known as (non-Cartesian) Controlled Aliasing in Parallel Imaging Results in Higher Acceleration (CAIPIRINHA) in the literature [152, 153] and will be studied in more detail in Chapter 5. Note that for fully sampled SMS acquisitions there is no temporal benefit compared to conventional multi-slice measurements.

The actual disentangling of slices in SMS acquisitions with interleaved trajectories happens naturally in the image reconstruction routine by the use of coil sensitivity information and is mathematically equivalent to the use of parallel imaging for in-plane undersampling. Therefore, a requirement for the successful application of SMS MRI is sufficient coil sensitivity variation in slice direction. More details on the reconstruction of SMS MRI measurements and a study of the benefits of various k-space trajectories for different slice-encodings can be found in Chapter 5 .

To achieve a Fourier-encoded SMS excitation for $M$ slices is straight forward. Let $\boldsymbol{B}_{\mathrm{rf}}^{(1)}\left(z_{q}, \Delta z\right)$ from Equation (2.35) be an RF pulse, which excites a single slice of thickness $\Delta z$ at slice-center location $z_{q}$. Then, the SMS excitation pulse for partition $p$ is the superposition of single-slice RF pulses modulated by the respective Fourier factors,

$$
\tilde{\boldsymbol{B}}_{\mathrm{rf}, p}^{(M)}\left(z_{1}, \ldots, z_{M}, \Delta z_{1}, \ldots, \Delta z_{M}\right):=\sum_{q=1}^{M} \Xi_{p q} \boldsymbol{B}_{\mathrm{rf}}^{(1)}\left(z_{q}, \Delta z_{q}\right) .
$$


To enable a convenient implementation on the MRI scanner, Equation (2.80) can be reformulated [63]. For the sake of simplicity, we assume a constant slice thickness $\Delta z$ and an equal inter-slice distance for all slices $q$. With the definitions

$$
\Omega_{c}:=\frac{\sum_{q=1}^{M} \omega_{c, q}}{M}, \quad \Delta \Omega_{q}:=\omega_{c, q}-\Omega_{c}
$$

where $\omega_{c, q}$ is the carrier frequency of slice $q$, we obtain ${ }^{7}$

$$
\begin{array}{cll}
\tilde{\boldsymbol{B}}_{p, x y}^{(M)}\left(z_{1}, \ldots, z_{M}\right) & \stackrel{\text { Eq.(2.36)(2.80) }}{=} & \sum_{q=1}^{M} \Xi_{p q} B_{1}^{(1)}(t) e^{-i \omega_{c, q} t} \\
& \stackrel{\text { Eq.(2.81) }}{=} & \left(B_{1}^{(1)}(t) \sum_{q=1}^{M} \Xi_{p q} e^{-i \Delta \Omega_{q} t}\right) e^{-i \Omega_{c} t} .
\end{array}
$$

Hence, a single carrier frequency $\Omega_{c}$ can be used in combination with a phasemodulated single-slice pulse envelope $B_{1}^{(1)}$ to achieve Fourier-encoded SMS excitation. A schematic of exemplary SMS RF pulse envelopes to excite four slices with Fourier-encoding according to the $4 \times 4$ Fourier matrix

$$
\Xi \stackrel{\operatorname{Eq} .(2.78)}{=}\left(\begin{array}{rrrr}
1 & 1 & 1 & 1 \\
1 & -i & -1 & i \\
1 & -1 & 1 & -1 \\
1 & i & -1 & -i
\end{array}\right)
$$

is given in Figure 2.10 (top row). The corresponding frequency domain representations (bottom row) illustrate the four separated frequency bands of the individual pulses. The respective phase of the bands represents the Fourier-encoding according to the rows of Fourier matrix Equation (2.83). Note that the imperfect (nonrectangular) frequency band profiles result from the finite pulse duration.

SMS excitation using a superposition of single-slice RF pulses comes with three major limitations. First, the superposition of single-slice excitation pulses leads to a linear increase of amplitude and consequently to a quadratic increase of the SMS excitation pulse peak power, which can exceed the capabilities of the RF amplifier. Second, the more slices are simultaneously excited, the more power is deposited in the subject, which potentially leads to violations of the SAR - particularly for sequences that require a high flip angle. Third, the Fourier-encoding scheme counteracts the necessary $\pm \pi$ RF pulse phase variation of bSSFP sequences and is therefore only applicable for FLASH sequences.

Various methods have been proposed to alleviate these limitations [154]. To prevent exceeding the peak power of the RF amplifier, the single-slice excitation pulses can additionally be modulated by a phase factor [155] or be applied in fast succession rather than at the same time [156, 157]. The total RF power restrictions can be

7 For convenience we drop the subscript ${ }_{\mathrm{rf}}$ and do not explicitly denote the dependency on $\Delta z$ and, accordingly, $\Delta \omega_{L}$. 


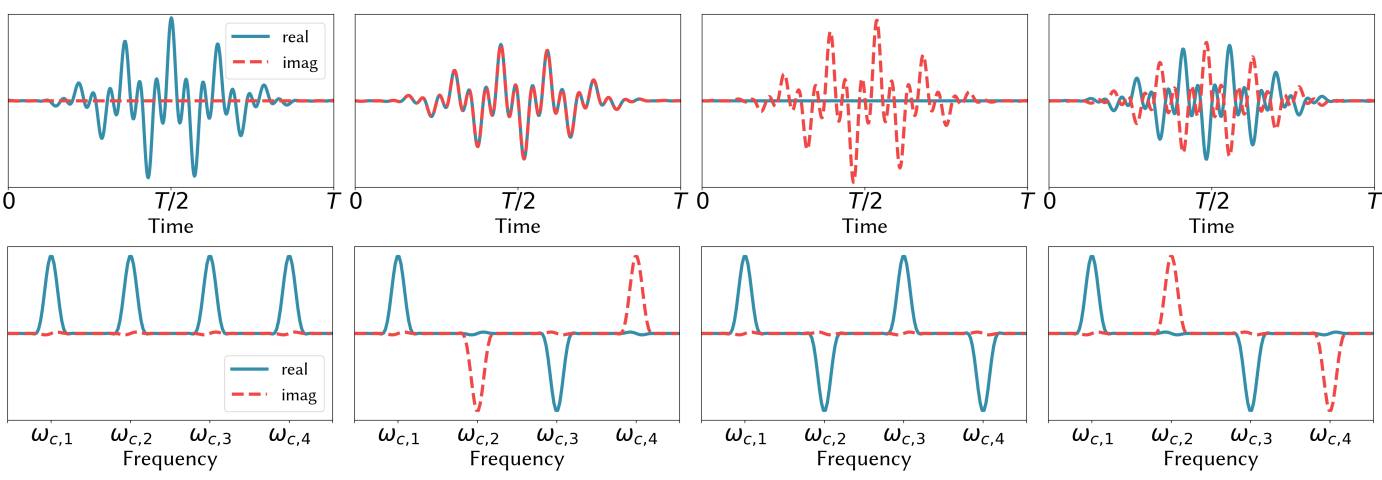

Figure 2.10.: Schematic illustration of the real and imaginary part of SMS RF pulse envelopes for the simultaneous excitation of four slices. (Top row) Time domain. (Bottom row) Frequency domain, where the imperfect (non-rectangular) frequency band profiles for slices $q$ with center frequencies $\omega_{c, q}$ are a consequence of the finite pulse duration $T$. The frequency offset due to the carrier frequency $\Omega_{c}$ is implicitly assumed. From left to right the different slice-encodings according to the rows of the Fourier matrix Equation (2.83) are depicted.

overcome using Variable-Rate Selective Excitation (VERSE): By reducing the amplitude of the slice-selection gradient at times of high energy deposition, the k-space is effectively traversed slower and the total RF power is reduced. Alternatively, Power Independent of Number of Slices (PINS) makes use of the Fourier convolution theorem and applies a sequence of short rectangular RF pulses with interleaved slice-selection gradients to generate the desired excitation, while the power requirement is independent of the number of slices. One way to implement SMS excitation for bSSFP is the use of a problem adapted phase-cycling scheme for the RF pulse [158], which comes at the expense of limited flexibility in the choice of the number of slices. A different and somewhat more natural approach is the use of phase-encoding gradients to induce the Fourier modulation $\Xi_{p q}$ in the slices [159].

Both the sequential multi-slice and the SMS approach can be seen as first steps towards the third spatial dimension in cardiac MRI. However, for many applications a complete three-dimensional time-resolved volume rendering of the beating heart is desired.

VOLUMETRIC ENCODING AND STACK-OF-STARS IMAging Volumetric encoding in MRI is a natural extension of two-dimensional imaging and is therefore based on the same signal equation (2.41). Volume-selective excitation follows the same principles as the slice-selective excitation described in Section 2.2.2. To distinguish two- and three-dimensional excitation, we commonly refer to slab-profiles in volumetric imaging compared to slice-profiles in single- and multi-slice imaging.

For three-dimensional signal encoding, two strategies can generally be distinguished: (1) The entire three-dimensional k-space is acquired using a non-Cartesian 
trajectory [160, 161]. (2) The third dimension of k-space - which is by convention defined as the $z$-dimension - is sampled on a rectilinear grid, i.e. on Cartesian coordinates, whereas the in-plane encoding is Cartesian or non-Cartesian [81, 162, 163].

Three-dimensional, purely non-Cartesian sampling with a koosh-ball [160] or phyllotaxis [161] trajectory acquires the three-dimensional k-space using radial spokes that all cross the k-space center. These trajectories can be seen as the natural extension of two-dimensional radial imaging. While providing high motion robustness and mild undersampling artifacts, the major problem with this kind of sampling is the huge amount of data, which is collected due to the equal size of all three spatial dimensions. Accordingly, the memory and computational demands are very high.

A promising alternative is the use of radial in-plane encoding and Cartesian encoding along the third dimension [162]. This so-called Stack-of-Stars sequence will be used in the context of Chapter 7. The SOS sequence combines several advantageous properties. Most importantly, the Cartesian dimension enables a flexible choice of the size of the third dimension, which can be used to better adjust the FOV to the actual geometry of the object and furthermore decreases the memory demands significantly. Compared to purely Cartesian sampling, SOS imaging maintains the milder undersampling artifacts and the motion robustness of radial imaging as described in Section 2.2.4 to a large extent. Finally, the consecutive acquisition of spokes from different partitions naturally gives rise to an $\mathrm{AC}$ region for direct cardiac and respiratory self-gating without the need for additional navigator lines [143], which will be discussed in Section 2.5.3 and demonstrated in Chapter 7. Recently, SOS undersampling in the Cartesian direction was proposed, which we will employ in Chapter 7 to improve the temporal resolution of the motion state estimate in self-gated cardiac imaging [164]. An illustration of these applications can be found in Figure 2.14b. Exemplary slices extracted from a fully sampled isotropic SOS bSSFP phantom experiment are depicted in Figure 2.11. ${ }^{8}$

Figure 2.12 depicts the pulse diagram of the SOS bSSFP sequence, which resembles Figure 2.5 for radial single-slice bSSFP imaging to a large extent. To resolve the third spatial dimension, a variable phase-encoding gradient $\left(B_{4}\right)$ is introduced, which will be discussed in more detail later. To comply with the requirements of bSSFP sequences, gradient $\left(B_{5}\right)$ ensures the zeroth gradient moment to vanish. In fact, the Fourier encoding of slices in SMS imaging can just as well be achieved using gradients [159]. Similar to SMS imaging, an interleaved trajectory for improved $\mathrm{k}$-space coverage is recommended for enhanced incoherence in compressed sensing reconstructions and to improve the overall image quality $[165,166]$.

8 Relevant measurement parameters corresponding to Figure 2.11 are: TE/TR $=2.35 / 4.70 \mathrm{ms,}$ flip angle $=33 \cdot 3^{\circ}, \mathrm{FOV}=192 \times 192 \times 144 \mathrm{~mm}^{3}$, voxel size $=1 \mathrm{~mm} \times 1 \mathrm{~mm} \times 1 \mathrm{~mm}$. 

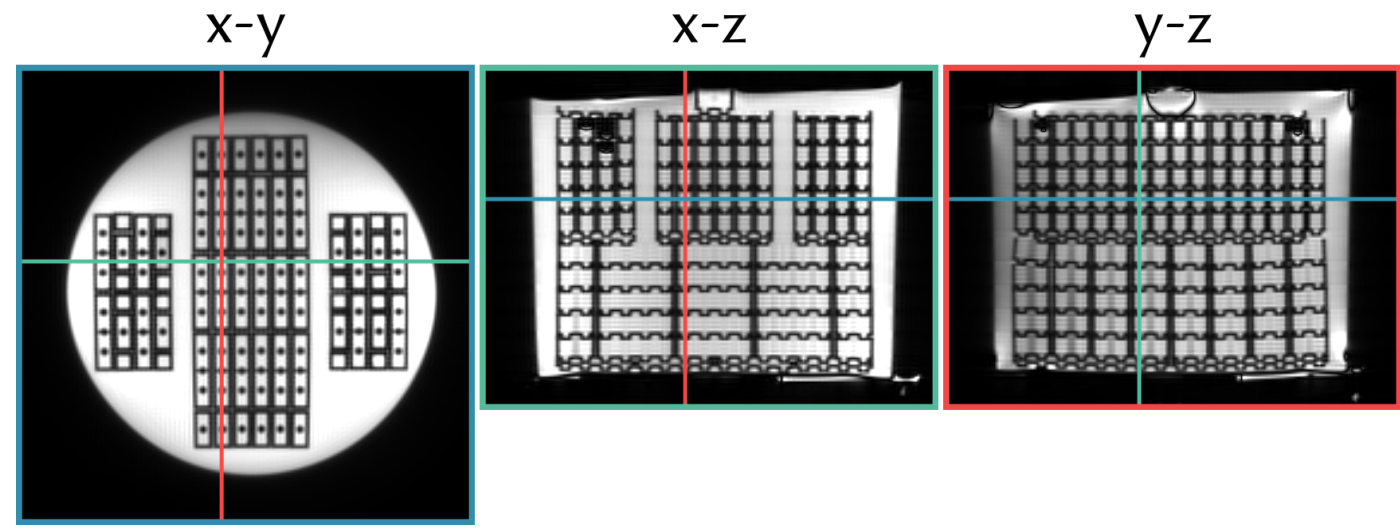

Figure 2.11.: Exemplary slices obtained from an isotropic Stack-of-Stars bSSFP measurement of the phantom depicted in Figure 5.3, which consists of plastic bricks immersed in water. Different views $(x-y-, x-z-$ and $y-z$-plane) are shown. The flexibility in the choice of the FOV in $z$-direction can be appreciated. The colored lines indicate the position of the different views.

To shorten the TR, gradients $\left(B_{2}\right)$ and $\left(B_{4}\right)$, as well as gradients $\left(B_{5}\right)$ and $\left(B_{3}\right)$ are commonly combined and played simultaneously with gradients $\left(C_{1}\right)$ and $\left(D_{1}\right)$ or $\left(C_{3}\right)$ and $\left(D_{3}\right)$, respectively.

Evidently, the extension of a given radial single-slice sequence to SOS imaging is straight forward and can essentially be realized by incorporating a phase-encoding gradient with variable gradient strength $G_{z}$. According to Equation (2.38), this phase-encoding gradient induces a linear phase-offset for the magnetization depending on the spatial position in $z$-direction

$$
\varphi\left(z, k_{z}\right)=z k_{z} \quad \text { with } \quad k_{z} \stackrel{\mathrm{Eq} \cdot(2.39)}{=} \int_{\mathrm{PE}} \gamma G_{z}(\tau) d \tau .
$$

In the latter equation, the integral covers the time for which the phase encoding gradient is active. By varying the gradient strength $G_{z}$ in different shots, a Fourierencoding of the signal in $z$-direction as per Equation (2.41) is achieved. Note that this Fourier-encoding is the continuous equivalent to Equation (2.77), where the RF pulse Equation (2.80) instead of the gradient is used to modulate the signal.

According to the Nyquist condition Equation (2.42), the sampling distance $\Delta k_{z}$ determines the FOV in $z$-direction. Therefore, particular attention must be devoted to the interaction of Cartesian phase encoding with an imperfect slab-profile, see Figure 2.2. This issue is schematically illustrated in Figure 2.13, where we assume an object which exceeds the FOV in $z$-direction. In the ideal case of a rectangular slab-profile (Figure 2.13a) only parts of the object within the desired FOV are excited and contribute to the acquired signal. Consequently, a Fourier-encoding 


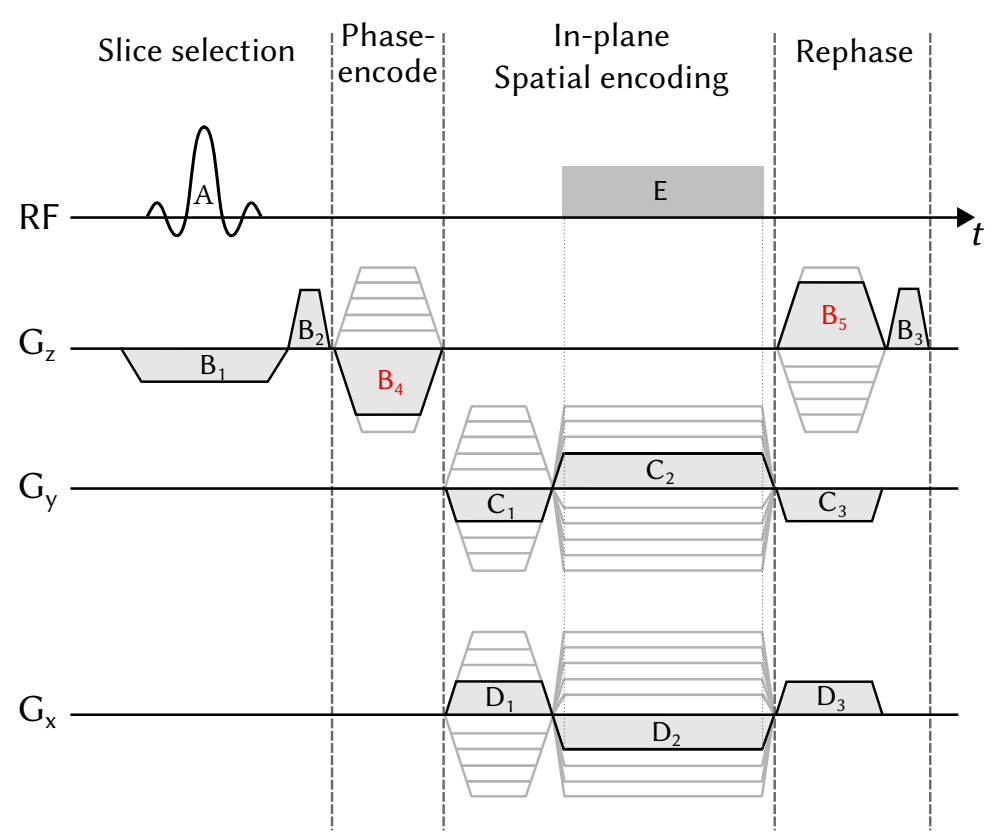

Figure 2.12.: Pulse diagram of a SOS bSSFP sequence. Compared to the radial singleslice bSSFP sequence, an additional gradient $\left(B_{4}\right)$ is used for phase-encoding of the Cartesian dimension. Gradient $\left(B_{5}\right)$ balances the zeroth gradient moment of the $z$-axis to zero. More details can be found in Figure 2.5.

with $\Delta k_{z}=1 / \mathrm{FOV}_{z}$ yields a signal which can be reconstructed without aliasing artifacts in $z$-direction. However, in real-world experiments the slab-profile will be smoothed, see Figure 2.2, thus parts of the object outside of the desired FOV will additionally be excited. Intuitively, the magnetization of these peripheral parts will experience excessive phase-offsets and will therefore be mislocated, or wrapped around, due to the ambiguity of the phase. As a result, the reconstructed image will suffer from aliasing artifacts (Figure 2.2b). To overcome this problem, the FOV in phase-encoding direction is commonly increased, while the slab profile remains unchanged. Therefore, the sampling distance $\Delta k_{z}$ must be decreased and more encodings steps $N$ are required to maintain the resolution $\Delta z=1 /\left(N \Delta k_{z}\right)$ (Equation (2.43)) in image space. After reconstruction, the intended image can be extracted by cropping the result to the desired FOV (Figure 2.2c). Contrary to readout oversampling mentioned in Section 2.2.4, oversampling in phase-encoding direction requires the acquisition of additional k-space lines and thus comes at the expense of an increased measurement time.

In this chapter we have focused on the bSSFP sequence, although all considerations hold for the FLASH sequence alike. However, unlike bSSFP, the FLASH sequence is not appropriate for volumetric cardiac MRI. In the latter, the steadystate magnetization of blood and myocardium is quite similar, which results in a 
(a) Ideal Case

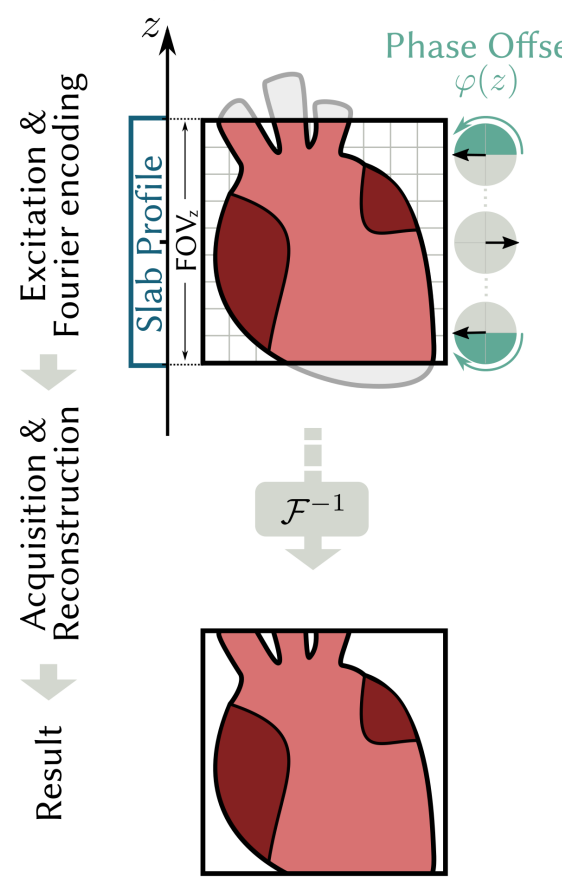

(b) Aliasing

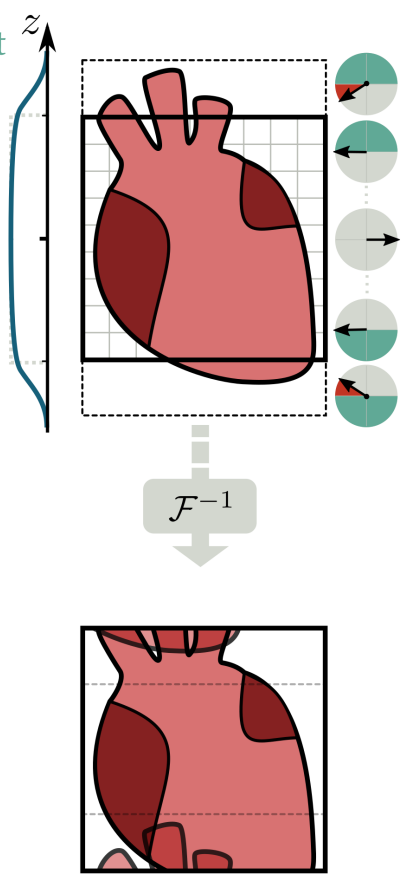

(c) Oversampling

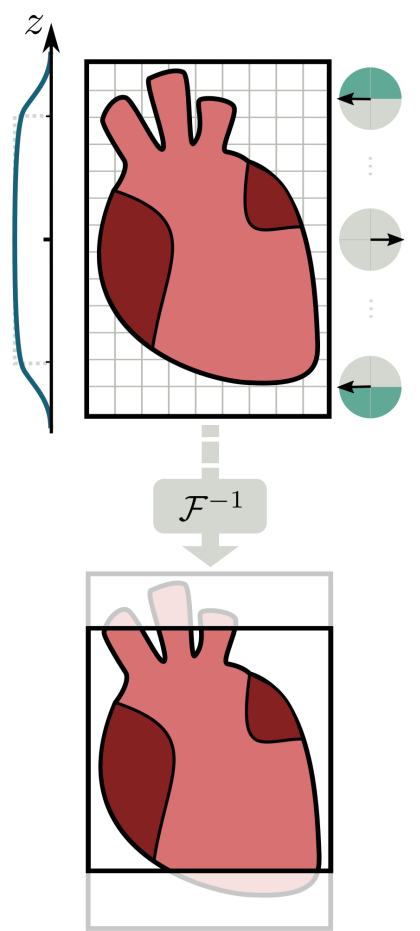

Figure 2.13.: Schematic illustration of the effects of (non-) ideal slab excitation profiles in combination with phase encoding in volumetric imaging. In (a), the investigated object exceeds the desired FOV in $z$-direction, but only the fraction of the object which is inside the FOV is excited due to the rectangular (ideal) slab excitation profile. To the right, the phase offsets $\varphi(z)$ (Equation (2.84)) for one Fourier-encoding step are depicted. While the phase is not changed at the center of the object, positive and negative offsets in the upper and lower parts are induced by a phase encoding gradient. Nyquist sampling according to $\Delta k_{z}=1 / \mathrm{FOV}_{z}$ yields an artifact-free result. In (b), an imperfect (realistic) slab profile excites parts of the object outside of the desired FOV. Those parts experience excessive phase offsets (highlighted in red), for which they are mislocated when sampling with $\Delta k_{z}=1 / \mathrm{FOV}_{z}$ is performed. The consequence is aliasing. In (c), oversampling is employed to eliminate the aliasing of (b). Therefore, the sampling distance $\Delta k_{z}$ is reduced while more encoding steps $N$ are performed to maintain the resolution $\Delta z=1 /\left(N \cdot \Delta k_{z}\right)$ in image space. This oversampling increases the FOV to incorporate all excited parts of the object. After reconstruction, the aliasingfree image can be cropped to obtain the desired FOV. 
poor image contrast. A distinct contrast between blood and myocardium in cardiac FLASH MRI relies on the inflow of fresh, unsaturated blood - a condition, which is fulfilled for single-slice, sequential multi-slice and, to a certain extend, SMS imaging. However, if a large portion of the heart gets excited as in volumetric MRI, fresh inflowing blood soon reaches the steady state, causing a severe loss of contrast in the systolic phase.

Note that apart from the distinct excitation, SOS and SMS MRI share many similarities. In fact, the reconstruction procedure for both approaches is vastly identical. One exception is that for the volumetric SOS acquisition a TV constraint in $z$-direction can be applied, which is not sensible for SMS imaging due to the nonvanishing slice gap.

\subsubsection{Exploiting Temporal Correlations}

Besides the unified processing of all spatial dimensions, time-resolved MRI enables the use of temporal correlations to speed up scans and to improve the image quality. However, most clinical approaches for cardiac MRI process each frame separately, thereby neglecting the fact that adjacent frames share many similarities. These similarities can be exploited during image reconstruction by means of temporal regularization, which can significantly reduce the data demand in dynamic MRI acquisitions.

In this section, we will present popular radial k-space trajectory schemes for time-resolved imaging and discuss related temporal regularization strategies for both dynamic and gated cardiac MRI.

RADIAL K-SPACE TRAJECTORIES FOR TIME-RESOLVED IMAGING In time-resolved imaging, we have to trade off k-space coverage against temporal-resolution. If we assign a large number of spokes to a single frame, undersampling effects are diminished. However, this will reduce the frame-rate and increase the risk to obtain blurry results, since spokes could be temporally too far apart to capture the same motion state. There is no comprehensive rule for how to choose the number of spokes per frame, as this will depend on the application. Nevertheless, the order of how spokes are acquired within and throughout different frames can significantly impact the achievable results. Here, we present two popular strategies for the design of k-space trajectories in time-resolved radial MRI.

The first scheme is illustrated in Figure 2.14, exemplarily for $N_{\mathrm{sp}}=5$ spokes per frame. This scheme features a uniform spoke distribution within a frame with projection angle increment $\alpha_{\mathrm{sp}}=2 \pi / N_{\mathrm{sp}}$. The projection angle varies from o to $360^{\circ}$, which has proved to reduce artifacts in certain experiments compared to a similar $\mathrm{k}$-space coverage with projection angles from o to $180^{\circ}$ [88]. 
To extend the k-space coverage, a different set of spokes can be used for subsequent frames. Therefore, the spoke pattern is rotated by $\alpha_{\text {fra }}=\alpha_{\mathrm{sp}} / N_{\text {pat }}$ with $N_{\text {pat }}=5$ as proposed in [42]. Accordingly, five consecutive frames will possess complementary spoke patterns and provide uniform k-space coverage with minimal gaps. These spoke patterns are repeated for succeeding frames. This scheme can be extended for time-resolved SMS imaging, as we will show in Section 6.1.

Another widely used radial trajectory is shown in Figure 2.14b, where the golden angle is employed as the projection angle increment for adjacent spokes,

$$
\alpha_{\mathrm{GA}}=\frac{2 \pi}{(1+\sqrt{5})} \approx 111.24^{\circ} .
$$

With the golden angle each new projection divides one of the largest azimuthal gaps in k-space by the golden ratio, which yields almost uniform k-space coverage at any point in time. This allows for temporal sliding window reconstructions with arbitrary numbers of spokes per frame, while still assuring nearly optimal k-space uniformity [167].

Another benefit of the golden angle scheme is the prevention of k-space redundancy, since no projection direction is acquired twice. Therefore, this strategy is ideally suited for gated imaging where spokes from different time-points are combined into bins.

One drawback of the classical golden angle scheme is the relatively large angle increment for adjacent spokes, which can cause artifacts for bSSFP sequences [168]. Therefore, Wundrak et al. proposed the use of tiny golden angles,

$$
\alpha_{\mathrm{GA}}^{N}=\frac{\pi}{(1+\sqrt{5}) / 2+N-1}
$$

where $N$ defines the tiny golden angle number. For $N=1$, Equation (2.86) recovers the classical golden angle.

The tiny golden angles comprise similar characteristics as the classical one, but possess a smaller angle increment [169, 170]. A popular choice, which will also be used later in this manuscript is the 7 th tiny golden angle $\alpha_{\mathrm{GA}}^{7} \approx 23.62^{\circ}$.

The golden angle schemes can naturally be extended to SMS and SOS imaging, which is illustrated in Figure 2.14b. Sequentially, one spoke is acquired for each partition, while a golden angle is used as projection angle increment. The central samples of such spoke stacks can be used to form an AC region, which allows for self-gated cardiac MRI, see Section 2.5.3. Note that the larger the number of partitions, the lower the temporal resolution of the AC region, which can lead to temporal inconsistencies within a spoke stack. Therefore, certain partitions in the periphery of the k-space can be skipped, while the energetically more dominant central partitions are acquired for all spoke stacks and used to construct the AC region [164]. We will apply this approach in Chapter 7 for self-gated SOS imaging. 
(a) Uniform Radial k-space Trajectory Schemes

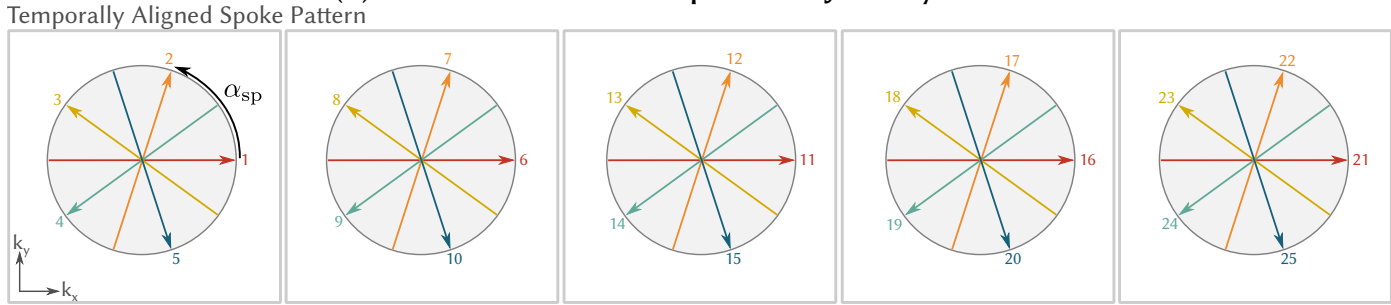

Complementary Spoke Pattern (Inter-Frame Rotations)

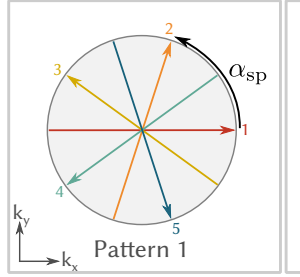

Golden Angle

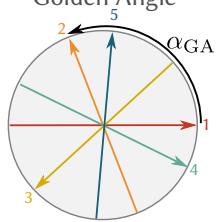

Tiny Golden Angle

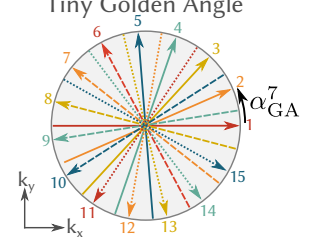

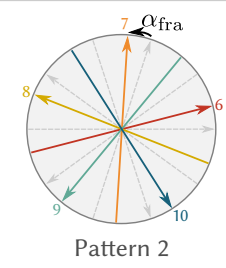

(b) Radial Golden Angle Trajectory Schemes

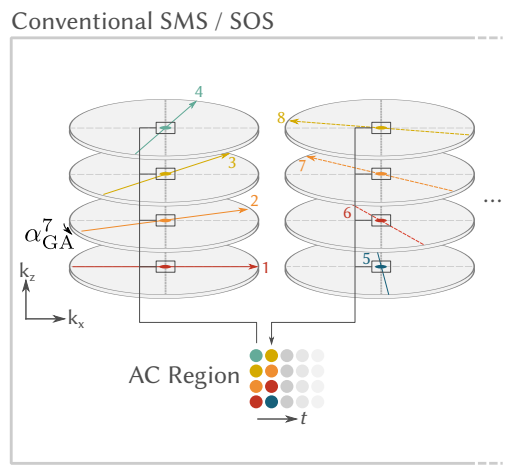

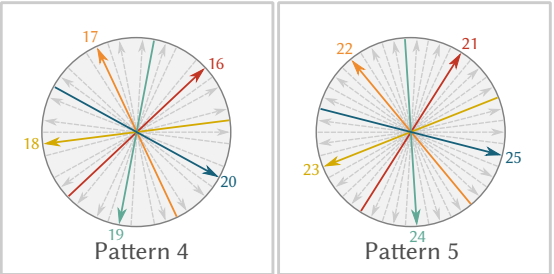

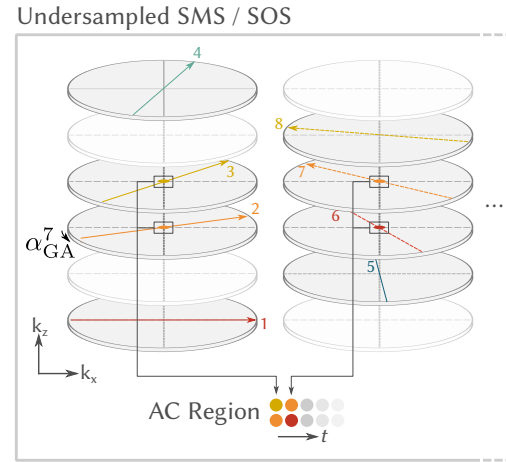

Figure 2.14.: Schematic of different radial k-space trajectories for time-resolved imaging. In (a), the $N_{\mathrm{sp}}=5$ spokes per frame are uniformly distributed by using the angle increment $\alpha_{\mathrm{sp}}=2 \pi / N_{\mathrm{sp}}$. In the top row, the spoke pattern is the same for all frames. In the bottom row, the spoke pattern is rotated in between frames by $\alpha_{\text {fra }}=\alpha_{\text {sp }} / N_{\text {pat }}$. Here, we choose a total of $N_{\text {pat }}=5$ different spoke patterns, which are repeated thereafter. The numbers next to the spokes indicate the acquisition order. The two schemes on the left side of $(b)$ show the spoke distribution using the golden angle $\alpha_{\mathrm{GA}} \approx 111.24^{\circ}$ (top) and the $7^{\text {th }}$ tiny golden angle $\alpha_{\mathrm{GA}}^{7} \approx 23.62^{\circ}$ as angle increment. The golden angle schemes can also be used for conventional and undersampled SMS and SOS experiments. If the partitions are iterated through for each TR, the central samples of the spokes from repeatedly acquired partitions form an AC region, which can be used for self-gated MRI. 
TEMPORAL REgUlarization AND MULTI-Temporal Dimensions Assuming sufficient temporal resolution, the changes between adjacent frames in timeresolved MRI are little. If those frames furthermore possess complementary kspace patterns, temporal regularization can be utilized to improve the image quality and reduce the data demand. ${ }^{9}$

The key idea behind temporal regularization is to enforce similarity between neighboring frames by penalizing large changes. For the sake of simplicity, we first consider a causal frame-by-frame reconstruction with Tikhonov-Phillips regularization, where the difference of the current and the previous frame is penalized. Inspired by Equation (2.52), the optimization problem for time-step $t$ can be formulated as

$$
\boldsymbol{x}^{*(t)}=\underset{\boldsymbol{x}}{\operatorname{argmin}}\left\|F \boldsymbol{x}^{(t)}-\boldsymbol{y}^{(t)}\right\|_{L_{2}}^{2}+\lambda\left\|\boldsymbol{x}^{(t)}-\boldsymbol{x}^{(t-1)}\right\|_{L_{2}}^{2} .
$$

Figure 2.15 demonstrates the effect of this regularization on reconstructions of a human heart with five spokes per frame. ${ }^{10}$ In Figure 2.15a, no temporal regularization was applied and the images exhibit strong undersampling artifacts. In Figure $2.15 \mathrm{~b}$, temporal regularization was applied but a temporally aligned spoke pattern was utilized, which yields no significant improvement compared to Figure 2.15a. In Figure 2.15c, temporal regularization was used in combination with complementary spoke patterns for different frames. Here, the first frame on the very left exhibits similar artifacts as the reconstructions from Figure 2.15a and Figure $2.15 \mathrm{~b}$ since no previous frame was available for temporal regularization. However, the subsequent frames show a striking improvement in image quality.

For its apparent impact on the reconstructions, temporal regularization is the backbone of modern time-resolved MRI. The proposed penalty term in Equation $(2.87)$ is the simplest and computationally most efficient type of temporal regularization. It can be used e.g. for real-time MRI, where the entire (ungated) dynamics of the motion is reconstructed and displayed with low latency on the scanner [43]. ${ }^{11}$ Other dynamic MRI techniques also recover the entire dynamics of the motion, but use a non-causal joint reconstruction involving all frames simultaneously. Here, a popular choice is the TV constraint, defined in Equation (2.55), employed on the temporal domain [174].

Temporal regularization can also be used for gated reconstructions, where periodic motion is not reconstructed in its full dynamics as in real-time MRI, but

9 While in this manuscript we will utilize temporal regularization, other approaches exist to exploit temporal redundancy for time-resolved imaging, e.g. [33, 171-173].

10 Note that NLINV was used to create the reconstructions shown in Figure 2.15 [40-42]. More details on the algorithm and the incorporation of temporal regularization can be found in Chapter 5 and Chapter 6.

11 Techniques for which the images are directly available on the scanner are frequently referred to as online methods, in opposition to offline methods, where the potentially time-consuming reconstructions are performed after the scan. 
(a) Independent Reconstruction

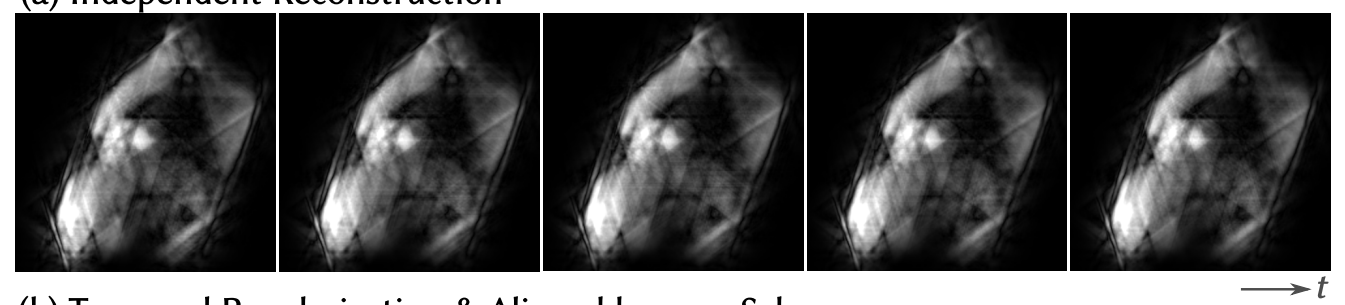

(b) Temporal Regularization \& Aligned k-space Scheme

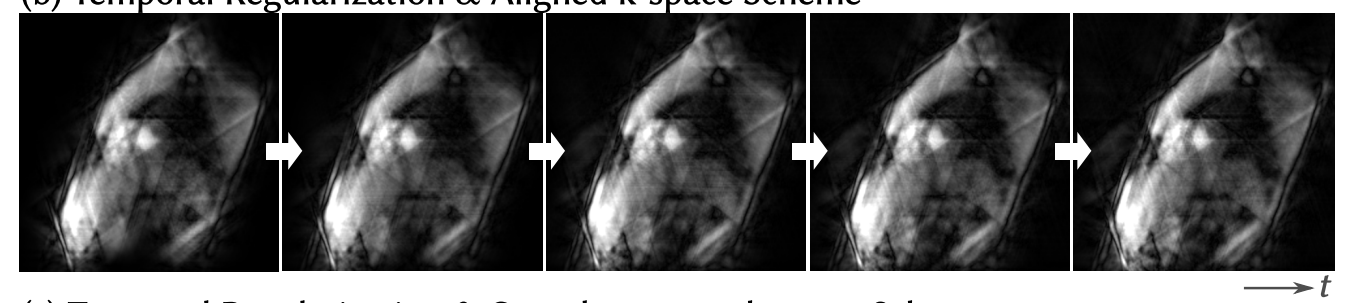

(c) Temporal Regularization \& Complementary k-space Scheme

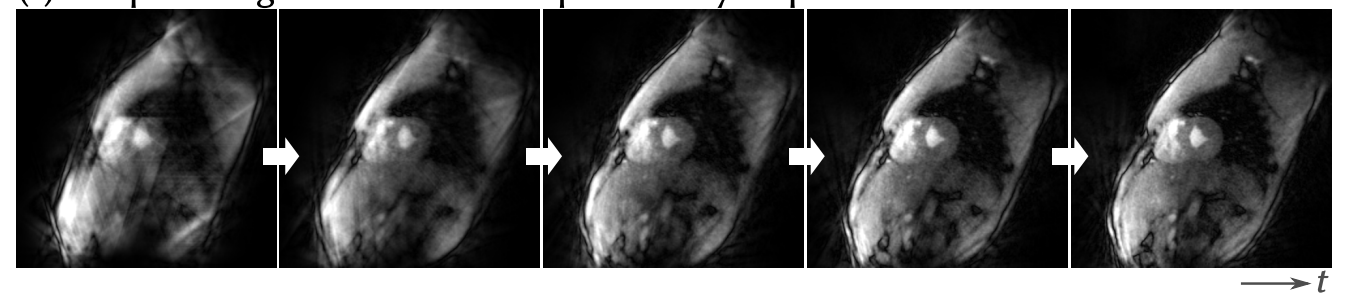

Figure 2.15.: NLINV reconstructions using five spokes per frame to demonstrate the effect of temporal regularization and different spoke patterns on time-resolved cardiac MRI. In (a), each frame is reconstructed individually and the same spoke pattern is utilized for all frames (compare Figure 2.14a, top row). In (b), on the same data temporal regularization, highlighted by the white arrows, is applied by penalizing the difference between the current and the previous frame. There is no visual improvement. In (c) we apply the same temporal regularization as in (b), but rotate the spoke pattern for each frame according to the bottom row of Figure 2.14a. For the first frame, no improvement can be observed, since no previous frame for regularization is available. For the subsequent frames, temporal regularization significantly enhances the results.

rather split up and binned into equivalent motion states. As a consequence, data is accumulated and a generic high-quality representation of a cycle can be generated. Compared to real-time imaging, where naturally only one temporal dimension exists, multiple temporal dimensions can be established in gated MRI. A typical example for free-breathing cardiac MRI is the use of a cardiac and a respiratory dimension as illustrated in Figure 2.16 and originally proposed in [143]. Like this, a temporal constraint can be imposed on both the cardiac and the respiratory dimension. Even though clinicians are only interested in a single respiration state, the multi-temporal approach allows for taking advantage of correlations from all acquired data. Hence, no data has to be dumped and the quality of the respiration state of interest is further improved. 
Multi-Dimensional Temporal Binning

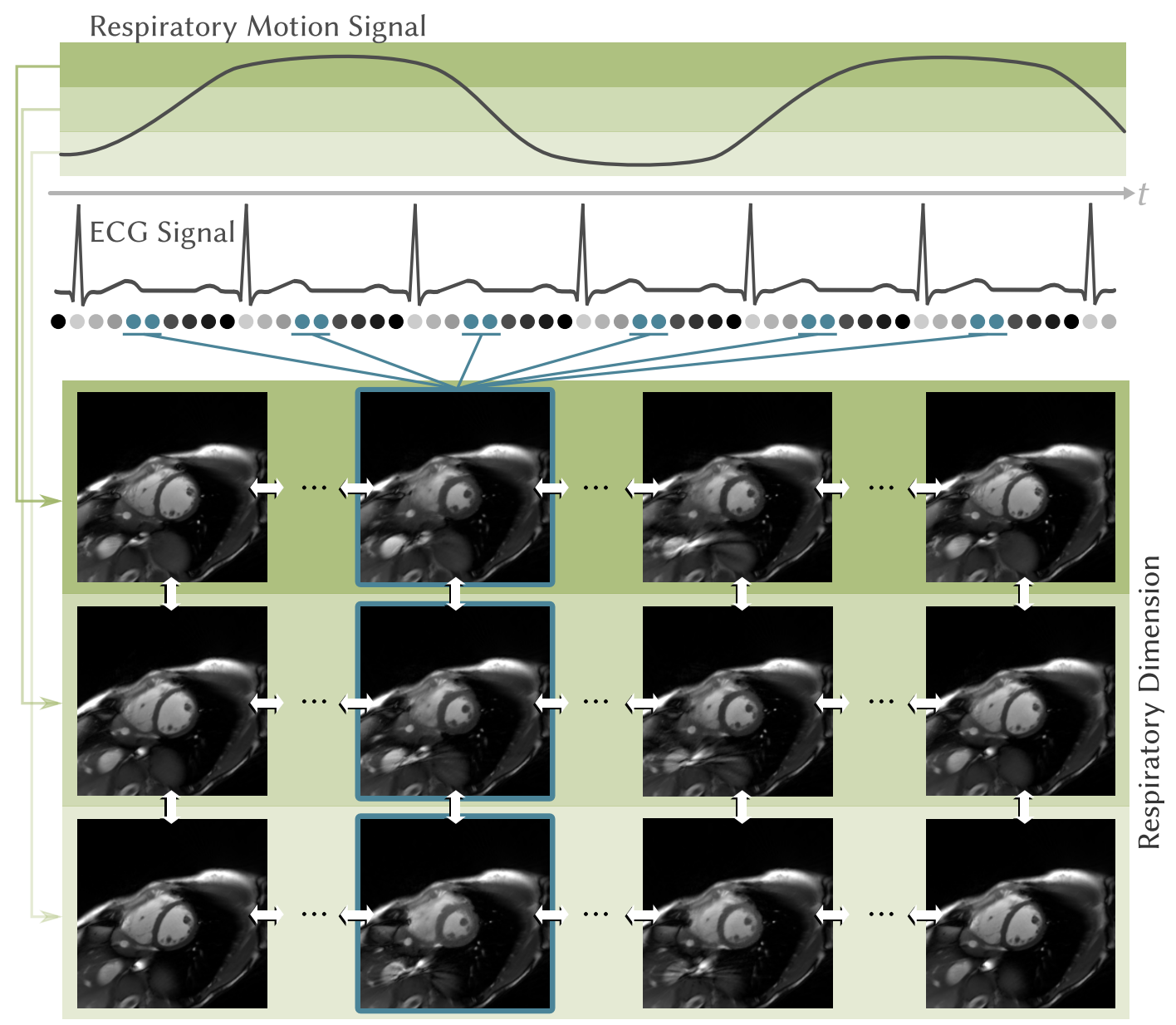

Cardiac Dimension

Figure 2.16.: Illustration of multi-temporal dimensions in free-breathing cardiac MRI. On top, the respiratory motion signal is divided into three respiratory bins and the cardiac motion state is detected e.g. using the ECG signal. Then, the data can be binned according to the cardiac and respiratory motion state. This generates two temporal dimensions, which can be exploited during image reconstruction by imposing e.g. a total variation constraint in both the cardiac and the respiratory dimension. The white arrows highlight correlated images, on which temporal regularization can be employed. 
This idea can be extended to even more temporal dimensions such as the change in imaging contrast for inversion-recovery based sequences [175]. The assignment of acquired data to bins of the potentially multi-temporal dimensions is a nontrivial task and will further be discussed in the next section.

\subsubsection{Self-Gating and the Concept of Time-Delayed Embedding}

In cardiac MRI we strive for a simple, fast and convenient data acquisition and at the same time demand robustness and high-quality reconstructions. Therefore, the ECG and a respiratory belt can be used to synchronize data acquisition with the cardiac and respiratory motion, which allows for the combination of data from several heartbeats to generate a single synthetic cardiac cycle. Gated cardiac MRI yields very good results, but the placement of external devices can be cumbersome and costs time. The monitoring of the respiratory motion can also be replaced by breath-hold commands, which, however, is inconvenient and problematic for non-compliant patients such as children, elderly or sick people. Alternatively, the steadily increasing computational power and the development of novel reconstruction strategies opened the door for real-time MRI, a technique which does not rely on patient cooperation and furthermore dispenses with the need for cardiac and respiratory gating. Data from multiple heartbeats are no longer combined but the acquired data is consistently used to reconstruct current images of the heart [29, 30, 42, 176]. Nevertheless, these real-time MRI techniques are limited to single-slice or SMS imaging with few slices. Furthermore, the combination of data from multiple heartbeats still tends to provide better results than dynamic imaging [61]. An interesting alternative to the aforementioned strategies is self-gated cardiac MRI.

SELF-GATED CARDIAC MRI Instead of relying on external monitoring hardware, in self-gated cardiac MRI information about the cardiac and respiratory motion is extracted from the acquired data itself. Over the past decades, a variety of approaches have been proposed to extract either the respiratory motion or the cardiac motion or jointly both motion signals from a suitable chosen AC region, e.g. [51, 52, 55, 57, 177].

Radial imaging is particularly appropriate for self-gating as it naturally provides an AC region. Each read-out line crosses the k-space center, thus its Direct Current (DC) component $\left(k_{x}=0, k_{y}=0\right)$ is sampled at a high temporal resolution. The value of the DC component represents the average brightness of the image and can directly be related to the current cardiac state: In end-diastole, the heart is filled with blood and therefore the image appears bright. During systole, the heart contracts and the average image brightness decreases. Figure 2.17 illustrates this principle. A similar reasoning holds for respiratory motion, hence a proper analysis 


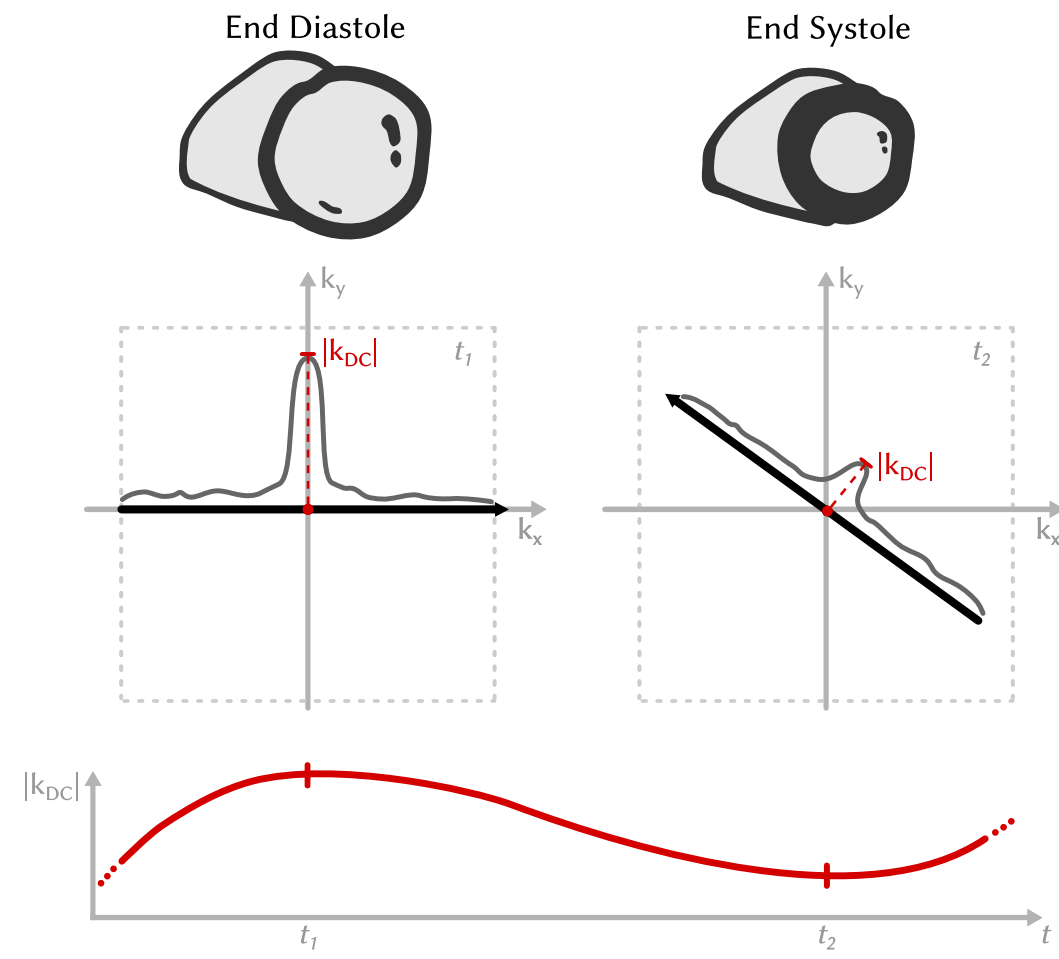

Figure 2.17:: Illustration of the relation between between the DC component $\left(k_{x}=\right.$ $\mathrm{o}, k_{y}=0$ ) of k-space and phases of the cardiac cycle. The top row shows a schematic of a heart in short-axis view for end-diastole and end-systole. Below, radial k-space lines (black) and corresponding magnitude values (grey) are shown for a sampling during end-diastole (time $t_{1}$ ) and end-systole (time $t_{2}$ ). Since the left-ventricular blood pool is larger at end-diastole, the average brightness - and likewise the value of the DC component $(\mathrm{red})$ - is higher compared to end-systole. The bottom row schematically shows the corresponding temporal evolution of the DC component, which can be extracted from each spoke and which contains information about the current cardiac phase.

of the temporal evolution of the DC component should reveal the cardiac and respiratory motion states.

For SMS or SOS acquisitions, the DC component $\left(k_{x}=0, k_{y}=0\right)$ of different partitions $k_{z}$ can be used jointly as AC region, which promotes a better detection of the motion signals. For illustration, compare Figure 2.14b.

Commonly, self-gating methods preprocess and band-pass filter the $\mathrm{AC}$ region to isolate the cardiac and respiratory motion signal, before a peak-detection algorithms is used to sort the data into their respective cardiac and/or respiratory phases. A more elegant method was proposed by Pang et al. who try to find the principle motion components by applying the PCA on the multi-channel AC region 
[56, 143]. Still, self-gating did not yet make its way into clinical practice, mainly due to the lack of robustness.

TIME-DELAYED EMBEDDING Here, we want to tackle this problem by approaching it from a different perspective. Instead of excavating the motion signals from the $\mathrm{AC}$ region by bruit force filtering or spatial dimensionality reduction, we consider the samples of the AC region as measurements of a potentially nonlinear dynamical system.

For a dynamical system the time evolution is defined in some phase space. A phase space is, simply speaking, a vector space where a point $s^{t}$ completely specifies the system's state at a certain time, discretely represented by the integer number $t$, with $0 \leq t<N_{t}$ and $N_{t}$ the total number of time-steps. The sequence of points $s^{t}$ is called the phase space trajectory. From the study of this trajectory, conclusions about the dynamics of the system can be drawn. Therefore, it is highly desirable to find a phase space representation of our dynamics. However, in experiments we usually cannot directly measure the phase space but a general time series - in our case the AC region extracted from our radial acquisition. Hence, we have to reconstruct a representation of the phase space.

We therefore fall back on a remarkable finding published by Packard et al. [178] in 1980, which states that, in principle, the full dynamics of a complicated nonlinear dynamical system - namely, its phase space - can be reconstructed from a single time series. This finding was soon thereafter formalized by Takens [179] and is today known as the method of delays or time-delayed embedding [180, 181]:

Let $x^{t}$ be the measured data at time-step $t$, which can be modeled by some measurement function $\mathcal{M}$ that processes the respective state $s^{t}$ of the system,

$$
\boldsymbol{x}^{t}=\mathcal{M}\left(s^{t}\right)
$$

A $W$-dimensional delay reconstruction can then be formed by stacking measurements $\boldsymbol{x}^{t}$ for different time-steps $t$ into a single $W \times\left(N_{t}-\tau(W-1)\right)$ delay vector $\boldsymbol{X}$, where the $t^{\text {th }}$ column of $\boldsymbol{X}$ is given by ${ }^{12}$

$$
\boldsymbol{X}^{t}=\left(\boldsymbol{x}^{t-w \tau}, \boldsymbol{x}^{t-(w-1) \tau}, \ldots, \boldsymbol{x}^{t}, \ldots, \boldsymbol{x}^{t+(w-1) \tau}, \boldsymbol{x}^{t+w \tau}\right)^{T}
$$

with $w:=(W-1) / 2$. In order to ensure $w$ to be integer, we assume $W$ to be an odd number. The lag or delay time $\tau>$ o determines the temporal difference between adjacent components of $\boldsymbol{X}^{t}$ and the quantity $\tau(W-1)$ defines the temporal window from which $\boldsymbol{X}^{t}$ is comprised of.

12 In the literature a time-delayed embedding is often defined by the combination of $x^{t}$ with past measurements only, $\boldsymbol{X}_{\text {past }}^{t}=\left(\boldsymbol{x}^{t-(W-1) \tau}, \boldsymbol{x}^{t-(W-2) \tau}, \ldots, \boldsymbol{x}^{t-\tau}, \boldsymbol{x}^{t}\right)^{T}$. However, to be consistent with Chapter 7 and for the ease of interpretation, in Equation (2.89) we choose a time-symmetric delay embedding. 
Commonly, the phase space trajectory $\boldsymbol{X}^{t}$ is not identical to the internal dynamics $s^{t}$ of the system. Still, under quite general assumptions, the reconstruction $\boldsymbol{X}^{t}$ created by a properly performed time-delayed embedding is guaranteed to be topologically equivalent to the full dynamics. In other words, there exists a uniquely invertible smooth function that maps $\boldsymbol{X}^{t}$ onto $s^{t}$ [180]. Many important measures of a dynamical system are invariant under this transformation, such as the Kolmogorov-Sinai entropy ${ }^{13}$, the fractal dimension of the invariant set ${ }^{14}$ or the Lyapunov exponents ${ }^{15}$.

Using the Rössler attractor [183] as an example, we will illustrate the equivalence of the original dynamics and a phase space reconstruction by time-delayed embedding. The Rössler system is defined by a set of three coupled nonlinear ordinary differential equations

$$
\frac{d}{d t} x=-y-z, \quad \frac{d}{d t} y=x+a y, \quad \frac{d}{d t} z=b+z(x-c) .
$$

In our example, we choose the constant parameter values $a=0.1, b=0.1, c=14$ and the initial conditions $x^{0}=-5, y^{0}=-5, z^{0}=0$. The top-left plot of Figure 2.18 shows the corresponding attractor. We measure the time evolution of the $x$-coordinate and reconstruct the phase space trajectory by applying Equation (2.89) with $W=3$, i.e. $w=1$, and different lag times $\tau=1,10,50$. The bottom row of Figure 2.18 shows the corresponding reconstructions. While the trajectory of the $\tau=1$ embedding is indistinguishable from that of a diagonal line when its extent is smaller than the measurement noise level, the $\tau=50$ reconstruction shows the same rise and twist behavior in the dimension orthogonal to the spiral plane as the Rössler attractor. Apparently, the choice of the embedding parameters has a distinct influence on the phase space reconstruction.

Takens original embedding theorem requires $W>2 D$, where $D$ is the integer dimension of the smooth manifold, which contains the attractor $s^{t}$. A generalization of this theorem by Sauer et al. [184] loosens this requirement to $W>2 D_{\mathrm{F}}$, with $D_{\mathrm{F}}$ the capacity or box counting dimension ${ }^{16}$ of the attractor, which can be significantly smaller than $D$ [180]. In some cases, attractors can even be reconstructed in spaces of dimension smaller than $2 D_{\mathrm{F}}$. Moreover, Takens theorem has very low

13 The Kolmogorov-Sinai entropy is a measure for the amount of information per unit time that is necessary to unambiguously determine the trajectory of a system [182].

14 Loosely speaking, an invariant set in dynamical system theory is a subset of the phase space. If we know that some part of the phase space trajectory is element of the invariant set at any time, we can conclude that the entire trajectory always was and ever will be element of this set. A fractal dimension is a generalization of the concept of dimension for geometric objects. One definition of a fractal dimension is the box counting dimension, see Footnote 16.

15 The Lyapunov exponent characterizes the rate of separation of infinitesimally close trajectories with time $[180]$.

16 The box counting dimension is defined as $D_{\mathrm{F}}:=\lim _{\epsilon \rightarrow 0} \ln (N(\epsilon)) / \ln (1 / \epsilon)$, where $N(\epsilon)$ is the number of boxes with edge length $\epsilon$ required to cover the attractor. 

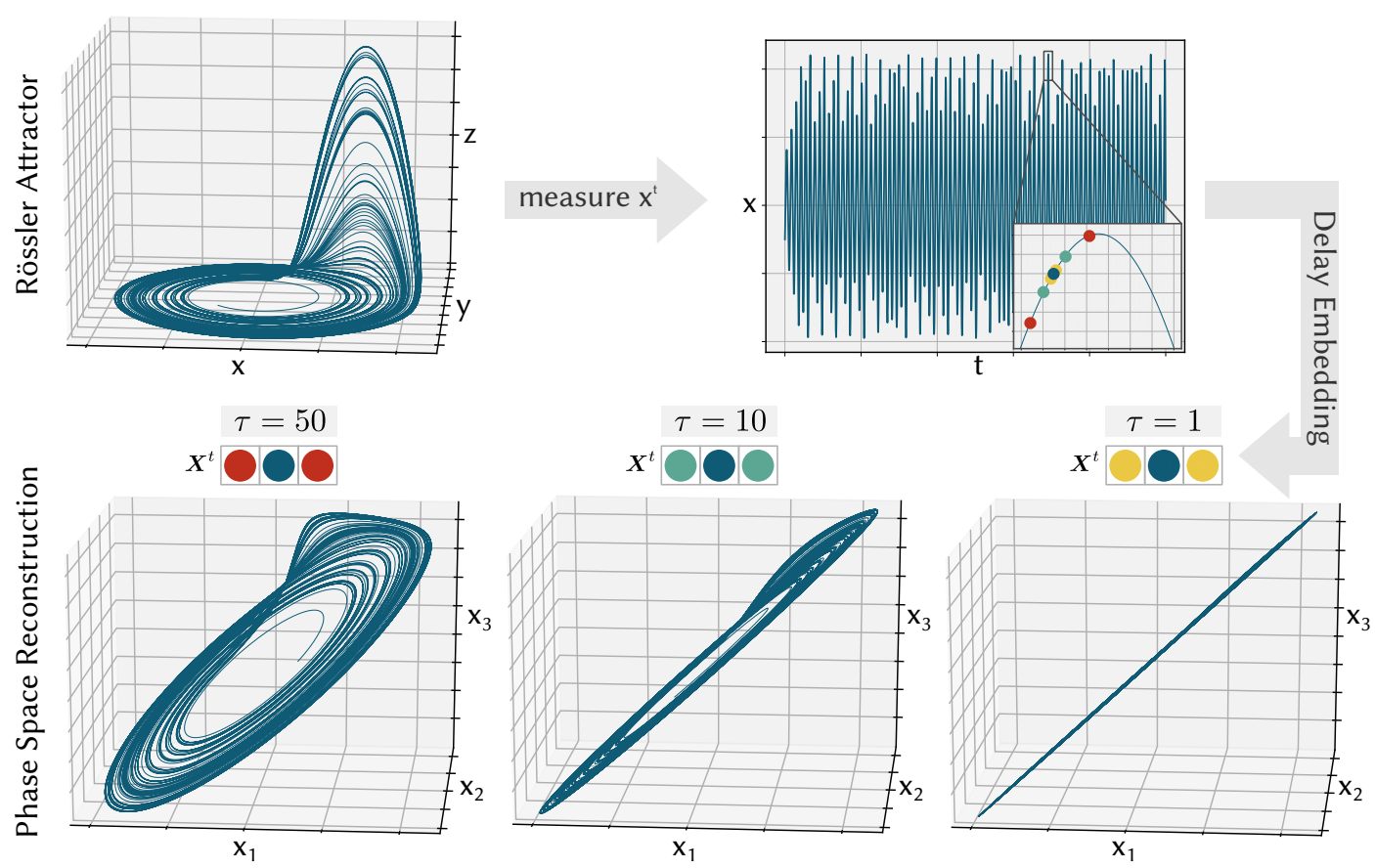

Figure 2.18.: Illustration of the phase space reconstruction of a Rössler attractor. On the top-left, the three-dimensional trajectory of the Rössler system is plotted. The topright shows the temporal evolution of the trajectory's $x$-coordinate. The zoomed inset plot schematically depicts a central sample (blue) and samples relatively shifted by $\tau=1$ (yellow), $\tau=10$ (green), $\tau=50$ (red). A $W=3$ dimensional delay vector with components $\boldsymbol{X}^{t}=\left(x^{t-\tau}, x^{t}, x^{t+\tau}\right)$ can be constructed by combination of the sample $x^{t}$ with its shifted neighbors. The bottom row shows the corresponding phase space trajectories for delay vectors with different values $\tau$. For a suitably chosen value, here $\tau=50$, the topology of the Rössler attractor or can be recovered.

restrictions on the choice of $\tau$, since it assumes infinite precise sampling. However, in real world applications a suitable choice of the product $\tau W$ is of major importance, as it determines the temporal window, which is represented by $\boldsymbol{X}^{t}$. For small values of $\tau W$, the $W$ coordinates of $\boldsymbol{X}^{t}$ are very similar, or redundant [185], and the trajectory consequently stretches along the main diagonal. With increasing $\tau W$, samples further apart in time are correlated and the trajectory unfolds off this subspace, revealing the underlying topology of the system. On the other hand, very large values for $\tau W$ are not necessarily always better as they can cause a reconstruction to fold over on itself [181]. More details on how to find good embedding parameters can be found in e.g. [180, 181].

In the context of MRI, we are not so much interested in the determination of the invariant measures of the dynamical system, but rather want to extract and separate the preferably noise-free signals of cardiac and respiratory motion from the $\mathrm{AC}$ region of our measurement. In Chapter 7 , we will point out how the theory 
of time-delayed embedding can be used to reach this goal. The main idea is to reconstruct a phase space trajectory $\boldsymbol{X}^{t}$ with parameters $\tau=1$ and a choice for $W$, which depends on the sampling rate of the AC region. Inspired by the SSA [186] we will show that performing a PCA on $\boldsymbol{X}^{t}$ resembles a data-adaptive filtering and denoising process of the original time series. This procedure can separate trend and noise from quasi-periodic motion, which makes it ideally suited for self-gated cardiac MRI.

\subsubsection{Conclusion}

A major goal of cardiac MRI is to image the beating heart and therefore the temporal evolution of a volumetric object. While classical approaches often neglect the nature of this problem, modern strategies try to exploit both spatial and temporal correlations to improve the image quality and speed up the measurement process.

Both SMS and SOS imaging translate the benefits of radial imaging into the third spatial dimension, which enables fast and efficient time-consistent imaging of multiple slices or volumes. Temporal regularization exploits similarities between neighboring frames to significantly reduce the data demand for the imaging of dynamic processes. Self-gated cardiac MRI does not require patient compliance and expedites patient preparation as it dispenses with additional hardware. The use of dynamical system theory to detect cardiac and respiratory motion from the measured data constitutes a promising alternative to the problem of self-gated cardiac MRI, potentially paving the way for a robust clinical application.

The presented methods will be employed in various ways throughout Chapters 3 to 7 . 
SIMPLE AUTO-CALIBRATED GRADIENT DELAY ESTIMATION FROM FEW SPOKES USING RADIAL INTERSECTIONS (RING)

The following is a reprint of the original article

S. Rosenzweig, H. C. M. Holme, and M. Uecker. "Simple auto-calibrated gradient delay estimation from few spokes using Radial Intersections (RING)". Magn. Reson. Med. 81 (2019), pp. 1898-1906.

(C) 2018 International Society for Magnetic Resonance in Medicine. Reprinted, with permission from [58].

$S R$ designed the RING method under supervision from $M U$ and implemented it with input from $C H$. SR designed and performed all simulations and experiments. $S R$ analyzed the results with guidance from $M U$. $S R$ wrote the majority of the manuscript with contributions by $\mathrm{MU}$ and $\mathrm{CH}$.

The layout, the reference numbering, the orthography and the use of acronyms have been adapted to preserve the natural text flow of this thesis. No substantive changes to the content have been realized.

In this article we introduce the RING method for gradient delay estimation and compare it to the AC-Adaptive method [133, 134], which is described in Appendix A. 


\section{ABSTRACT}

PURPose: To develop a simple and robust tool for the estimation of gradient delays from highly undersampled radial k-space data.

THEORY: In radial imaging gradient delays induce parallel and orthogonal trajectory shifts, which can be described using an ellipse model. The intersection points of the radial spokes, which can be estimated by spoke-byspoke comparison of k-space samples, distinctly determine the parameters of the ellipse. Using the proposed method (RING), these parameters can be obtained using a least-squares fit and utilized for the correction of gradient delays.

Methods: The functionality and accuracy of the proposed RING method is validated and compared to correlation-based gradient-delay estimation from opposing spokes using numerical simulations, phantom and in vivo heart measurements.

RESULTS: In all experiments, RING robustly provides accurate gradient delay estimations even for as few as three radial spokes.

Conclusion: The simple and straightforward to implement RING method provides accurate gradient delay estimation for highly undersampled radial imaging. 


\subsection{INTRODUCTION}

In today's clinical practice almost all Magnetic Resonance Imaging (MRI) techniques are based on Cartesian trajectories. Nevertheless, in the recent years non-Cartesian trajectories, in particular radial acquisitions, drew increasing interest among the scientific community for their motion robustness and milder undersampling artifacts and proofed potential for a significant imaging speed-up [41, 162, 187-189]. Although the benefits of radial trajectories seem evident, for various reasons they did not yet find widespread application in clinical routine. One reason is the higher sensitivity to system imperfections such as eddy-current-induced gradient delays, that lead to deviations from the nominal sampling locations [50]. To tackle this problem, a variety of different trajectory error compensation strategies have been developed.

One approach is to measure the actual k-space trajectory during each measurement using specialized hardware, which is highly effective but expensive and not always practical [190, 191]. Alternatively, calibration scans can be utilized to fully characterize the Gradient System Impulse Response Function (GIRF) of the scanner [192-195]. However, as these methods have not yet been adopted by the vendors they require significant implementation efforts and are difficult to integrate into a complete workflow. Furthermore, GIRF-based methods cannot capture sequence or protocol dependent temporal variations of the gradient system, for example, through heating, and the characterization must be repeated regularly to compensate long-term system variances.

Other approaches have been designed particularly for error compensation in radial imaging. Iterative, parallel imaging-based methods developed by Deshmane et al. [196] and Wech et al. [197] exploit correlations in the receive channels using GRAPPA operator gridding [198] and shift the samples or the trajectory position until a certain condition is fulfilled. Both methods are data driven and therefore can be used to retrospectively detect and compensate transient trajectory errors. These methods require good estimates for the gridding operators, which in turn demands sufficient in-plane coil sensitivity variation and a certain number of spokes for auto-calibration. These conditions might not always be given, as for example, in interactive real-time MRI the imaging plane is repeatedly being rotated and shifted [46, 199-201]. Recently, Jiang et al. [202] introduced a framework, which simultaneously estimates gradient delays and coil sensitivities using an alternating minimization approach. This method inspired by SAKE [203] uses a computationally rather demanding low-rank constraint in conjunction with the Gauss-Newton method to solve a nonlinear optimization problem. Furthermore, two more general algorithms have been proposed that combine trajectory correction and image reconstruction [204, 205]. However, in the approach of Mani et al. [204] prior knowl- 
edge about the coil sensitivities is necessary. Moreover, both algorithms might be difficult to combine with other image reconstruction methods.

A noticeable alternative to the above mentioned rather elaborate trajectory correction techniques is the Adaptive method developed by Block and Uecker [133] for radial imaging. Because of its intuitive approach, its robustness and accuracy and the straight forward implementation it found widespread acceptance and application [126, 134, 162, 170, 206]. The method requires calibration scans where pairs of opposed spokes with varying orientation are acquired. Then, the sample shift along the readout direction is calculated by performing a cross-correlation of the opposed spokes. The shifts are fitted to a linear ellipse model [50, 126] and compensated for in the gridding procedure. Recently, it was shown that the same approach can be used to estimate the gradient delays from the data itself without the need for calibration scans. This auto-calibrated variant, here dubbed AC-Adaptive method, was described by Untenberger et al. [134], applied in several publications, e.g. [44, 207, 208], and studied in [209]. Instead of using perfectly anti-parallel spokes, the AC-Adaptive method takes spokes from the actual radial acquisition which are only approximately anti-parallel to estimate the shifts. This allows for real-time and retrospective gradient delay correction, which can be applied to, for example, interactive real-time MRI or to compensate gradient delay changes due to coil heating. However, the present work shows that the AC-Adaptive method is not fully consistent with the ellipse model, which it is based on. In settings with oblique slices and/or non-isotropic delays of the physical gradients, the spokes will experience both a shift in read-out direction and orthogonal to it. Due to the latter, even perfectly opposed spokes will not cover the same k-space line, which leads to inconsistencies in the cross-correlation calculation. Furthermore, the AC-Adaptive method cannot provide stable gradient delay estimates given only very few spokes, as then no nearly opposed spoke pairs exist.

The aim of this work is to develop a simple yet accurate and robust gradient delay estimation tool, which also works for very few spokes. We show that our method, which uses Radial spoke INtersections for Gradient delay estimation (RING), outperforms the AC-Adaptive method in all investigated numerical simulations, phantom and in vivo heart measurements and provides precise gradient delay estimates even for as few as 3 spokes.

\subsection{THEORY}

THE GRADIENT DELAY ELLIPSE MODEL. Peters et al. showed that in radial imaging, linear eddy current effects delay the start of the readout gradients, which induces both a parallel and an orthogonal shift to the nominal k-space trajectory, 
while the projection direction is not affected [50]. Moussavi et al. [126] proposed a simple model to describe this spoke shift as a vector

$$
\begin{gathered}
\delta \boldsymbol{k}:=\boldsymbol{S} \hat{\boldsymbol{n}}_{\theta}, \\
\hat{\boldsymbol{n}}_{\theta}:=\left(\begin{array}{c}
\cos \theta \\
\sin \theta
\end{array}\right), \\
\boldsymbol{S}:=\left(\begin{array}{cc}
S_{x} & S_{x y} \\
S_{x y} & S_{y}
\end{array}\right)
\end{gathered}
$$

with $\theta$ the projection angle and $\hat{\boldsymbol{n}}$ the normalized projection direction. $S_{x}$ and $S_{y}$ capture the delays in the axial case whereas $S_{x y}$ guarantees three-dimensional rotational invariance and particularly accounts for the interaction of all three physical gradients when measuring oblique slices. For a detailed derivation please consider Section 3.8. The goal of this work is to efficiently determine the parameters $S_{\mathrm{x}}, S_{\mathrm{y}}$ and $S_{x y}$, which can then be used to calculate the actual (shifted) trajectory needed for accurate gridding in image reconstruction.

Let $N_{\text {samp }}$ be the number of samples in readout direction and $N_{\text {sp }}$ the number of spokes used for gradient delay estimation. Then, the sample positions in units of I/FOV of a spoke with projection angle $\theta_{i}, i \in\left\{1, \ldots, N_{\mathrm{sp}}\right\}$, can be modeled using the parametric linear equation

$$
\boldsymbol{r}_{\theta_{i}}=\boldsymbol{S} \hat{\boldsymbol{n}}_{\theta_{i}}+a_{\theta_{i}} \hat{\boldsymbol{n}}_{\theta_{i}}
$$

with $a_{\theta_{i}}=\left[-N_{\text {samp }} / 2, N_{\text {samp }} / 2-1\right]$. The ellipse defined by Equation (3.1) determines the position $\boldsymbol{r}_{\theta_{i}}\left(a_{\theta_{i}}=0\right)$ of the shifted spokes.

Figure 3.1 depicts a schematic of actual k-space trajectories for different delays $\boldsymbol{S}$. If no gradient delays are present (top-left) the spokes are not shifted at all. For isotropic delays in the axial case (top-right) the spokes are translated in readout direction only. For anisotropic delays (bottom-left) and/or oblique slices (bottomright) the spokes experience both a readout-shift and an orthogonal shift and no longer intersect in the k-space center. The intersection points of the spokes relative to their DC component uniquely define the shift matrix $S$.

Determination of $S_{x}, S_{y}$ and $S_{x y}$. The intersection point of the spokes $\boldsymbol{r}_{\theta_{i}}$ and $\boldsymbol{r}_{\theta_{j}}$ yield a conditional equation for $S$ :

$$
\begin{gathered}
\boldsymbol{r}_{\theta_{i}}\left(a_{\theta_{i}}^{\prime}\right) \stackrel{!}{=} \boldsymbol{r}_{\theta_{j}}\left(a_{\theta_{j}}^{\prime}\right), \\
\boldsymbol{S}\left(\hat{\boldsymbol{n}}_{\theta_{i}}-\hat{\boldsymbol{n}}_{\theta_{j}}\right)=a_{\theta_{j}}^{\prime} \hat{\boldsymbol{n}}_{\theta_{j}}-a_{\theta_{i}}^{\prime} \hat{\boldsymbol{n}}_{\theta_{i}} .
\end{gathered}
$$

To facilitate calculations, we introduce the definitions

$$
s:=\left(\begin{array}{c}
S_{x} \\
S_{y} \\
S_{x y}
\end{array}\right),
$$




$$
\begin{aligned}
\hat{\boldsymbol{n}}_{\theta_{i}}-\hat{\boldsymbol{n}}_{\theta_{j}}:=\left(\begin{array}{c}
\xi_{1} \\
\xi_{2}
\end{array}\right), \boldsymbol{A}:=\left(\begin{array}{ccc}
\xi_{1} & 0 & \xi_{2} \\
0 & \xi_{2} & \xi_{1}
\end{array}\right), \\
\boldsymbol{b}:=a_{\theta_{j}}^{\prime} \hat{\boldsymbol{n}}_{\theta_{j}}-a_{\theta_{i}}^{\prime} \hat{\boldsymbol{n}}_{\theta_{i},}
\end{aligned}
$$

and rearrange Equation (3.6)

$$
A s=b .
$$

If $N_{\mathrm{sp}}>2$, Matrix $\boldsymbol{A}$ and vector $\boldsymbol{b}$ can be extended to contain all considered intersection points. Then, the system of equations Equation (3.10) for $s$ is overdetermined and $s$ can be obtained by a least-squares fit using the pseudo-inverse

$$
s=\left(A^{T} \boldsymbol{A}\right)^{-1} \boldsymbol{A}^{T} \boldsymbol{b},
$$

where ${ }^{T}$ indicates the transpose.

DETERMinAtion of INTERSECTION POINTS. To obtain $s$ (Equation (3.11)) the values $a_{\theta}^{\prime}$ in Equation (3.6) must be determined from the measured data. Since the k-space value at the intersection point of two spokes should be identical except for noise in all channels, the values for $a_{\theta}^{\prime}$ can be obtained by comparing the actual complex sample values of the spokes. The sample pair for which the Root-Sum-ofSquares (RSS) difference over all channels is minimal is assumed to represent the intersection point. To guarantee accurate estimates for $a_{\theta}^{\prime}$ and thus $s$, each spoke is retrospectively sub-sampled via Fourier interpolation and denoised: An inverse Fourier transform is used to obtain a spoke's image domain representation. As the readout direction is generally oversampled by a factor of 2, all samples outside of the central $N_{\text {samp }} \times 0.6$ region can be set to zero to sinc-denoise k-space. Then, the data is zero-padded by $N_{\text {pad }} \times N_{\text {samp }}$ (we propose $N_{\text {pad }}=100$ ) and the sub-sampled $\mathrm{k}$-space is retrieved using another Fourier transform. As most of the energy is localized in the low spatial frequency region it is sensible to investigate only intersection points in the central region of k-space, which avoids inaccuracies due to noise. Therefore, only the intersection point of a spoke with its most orthogonal counterpart is considered, i.e. we search for the spoke pairs that best approximate an intersection angle of $90^{\circ}$. Then, the sample-wise comparison can be restricted to the central $\beta \times N_{\text {pad }}$ (we propose $\beta=1.5$ ) samples of the spokes.

An analysis of the accuracy of the proposed method to determine the intersection points for different noise values and simulated phantoms is provided as supplementary material (Figure 3.5). 


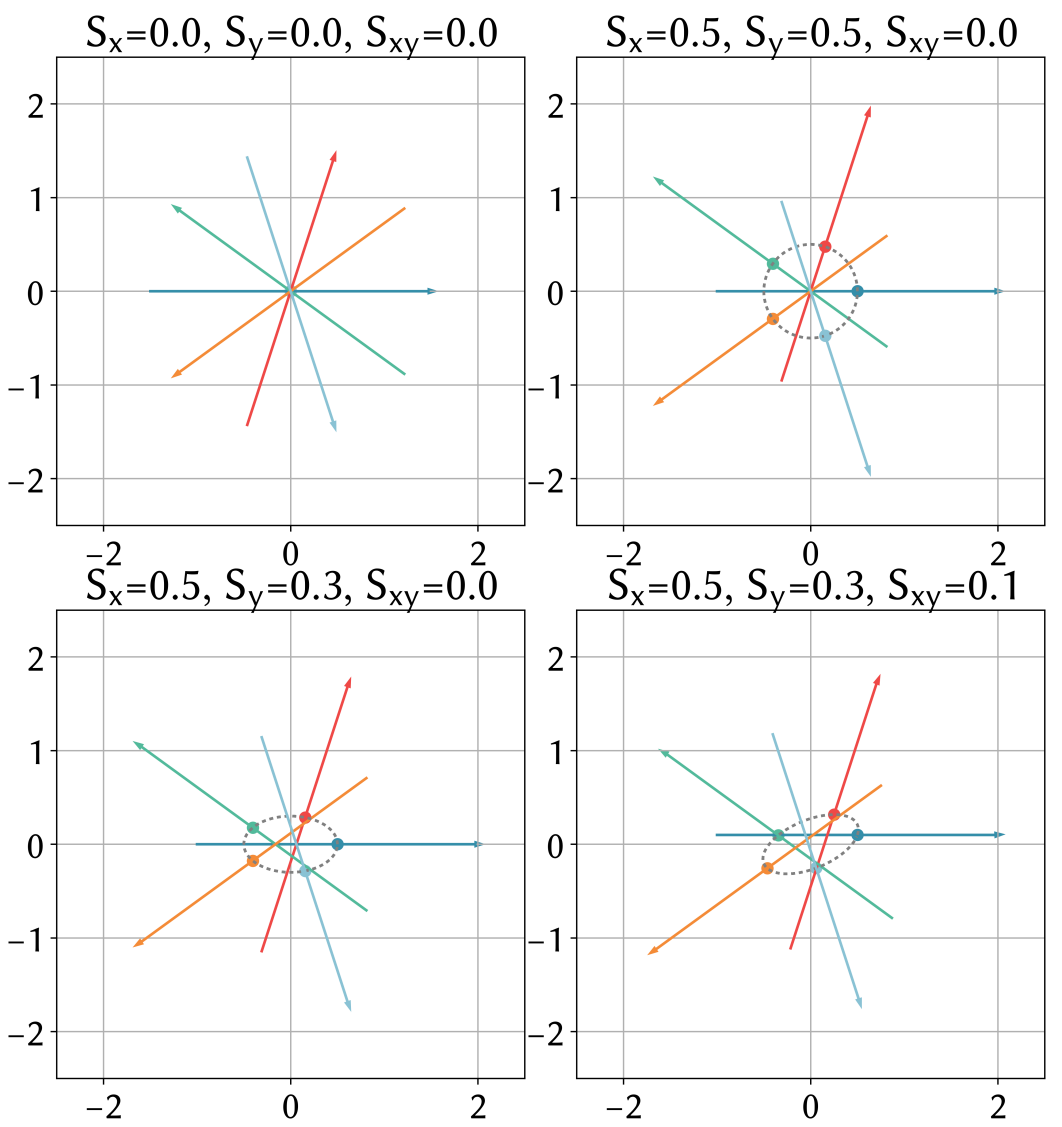

Figure 3.1.: Schematic of shifted k-space trajectories in units of $1 /$ FOV for different delays $S_{\mathrm{x}}, S_{\mathrm{y}}$ and $S_{\mathrm{xy}}$. Exemplarily, five abridged spokes are depicted. The arrows point in readout direction and the DC sample of each spoke is highlighted by a dot. The ellipse determined by $\delta \boldsymbol{k}$ (Equation (3.1)) is plotted as a dashed gray circle.

\section{$3 \cdot 3$ METHODS}

All measurements were performed on a SIEMENS Skyra $3 \mathrm{~T}$ scanner, all reconstructions and the gradient delay estimation methods were implemented and performed using BART [210]. In our study we chose the RING parameters to be $N_{\text {pad }}=100$ and $\beta=1.5$, which provided accurate and robust results throughout all experiments. In the interest of reproducible research, code and data to reproduce the experiments are made available on Github. ${ }^{1}$

NUMERical simulations. To demonstrate the general functionality and accuracy of our method, we performed a numerical Shepp-Logan k-space phantom study (oversampled readout samples 128, 8 channels) using a golden angle

1 https://github.com/mrirecon/RING 
scheme and spokes shifted according to Equation (3.1) with the nominal values $s^{\text {iso }}=(0.3,0.3,0), s^{\text {ax }}=(0.3,-0.1,0)$ and $s^{\text {obl }}=(0.3,-0.1,0.2)$ respectively.

To quantify the estimation error $\mathcal{E}\left(s, s_{N_{\mathrm{sp}}}^{\text {est }}\right)$ we used the L2 norm

$$
\mathcal{E}\left(s, s_{N_{\mathrm{sp}}}^{\mathrm{est}}\right):=\sqrt{\left(S_{x}-S_{x}^{\mathrm{est}}\right)^{2}+\left(S_{y}-S_{y}^{\mathrm{est}}\right)^{2}+\left(S_{x y}-S_{x y}^{\mathrm{est}}\right)^{2}},
$$

where $S$ stands for the nominal and $S^{\text {est }}$ for the estimated shifts. We estimated the shifts using RING for all numbers of spokes in the range $N_{\mathrm{sp}} \in[3,127]$ and performed the simulations for projection angles distributed over a half circle $\left(\theta_{i} \in\right.$ $[0, \pi])$ and a full circle $\left(\theta_{i} \in[0,2 \pi]\right)[211]$.

For comparison we did the same experiments using the AC-Adaptive method.

PHANTOM MEASUREMENTS. For the measurement on a custom-made brick phantom we used the SIEMENS Head-Neck-20 coil and a FLASH sequence (FOV $=256 \times 256 \mathrm{~mm}^{2}$, oversampled readout samples $=320$, number of spokes $=159$, $\mathrm{TE} / \mathrm{TR}=1.46 / 2.3 \mathrm{~ms}$, slice thickness $=5 \mathrm{~mm}$ ) with golden angle (half and full circle) acquisition. For the sake of better visibility of gradient delay artifacts, only 39 spokes were used for image reconstruction. The delays were estimated for all numbers of spokes in the range $N_{\mathrm{sp}} \in[3,159]$ using both RING and the AC-Adaptive method for full circle acquisitions. For half circle acquisitions only RING is utilized. We used radial NLINV [40, 41] in combination with the corrected trajectories for image reconstruction.

For each number of spokes $N_{\mathrm{sp}}$ the estimated delays $s_{N_{\mathrm{sp}}}^{\text {est }}$ were compared to $s_{159}^{\text {est }}$ and the L2 errors $\mathcal{E}\left(s_{159}^{\text {est }}, s_{N_{\mathrm{sp}}}^{\mathrm{est}}\right)$ according to Equation (3.12) were calculated.

IN VIVO MEASUREMENTS. We performed an in vivo measurement on a human heart (short-axis view, 30 channel thorax and spine coil, FLASH sequence, FOV $=256 \times 256 \mathrm{~mm}^{2}$, oversampled readout samples $=320$, TE $/ \mathrm{TR}=1.47 / 2.3 \mathrm{~ms}$, slice thickness $=8 \mathrm{~mm}$ ) using a full circle golden angle acquisition scheme. Seventy-five consecutive spokes during the end-diastole were combined for image reconstruction with ENLIVE [212]. The gradient delays were estimated using RING and the AC-Adaptive method utilizing all numbers of spokes in the range $N_{\mathrm{sp}} \in[3,75]$. The L2 errors were calculated as described previously.

Human imaging was approved by the local ethics committee. Written informed consent was obtained from the subject before the imaging.

\subsection{RESULTS}

NUMERICAL SIMULATIONS. The results of the numerical simulations are depicted in Figure 3.2. The error $\mathcal{E}\left(s, s_{N_{\mathrm{sp}}}^{\text {est }}\right)$ of the estimated gradient delays for differ- 

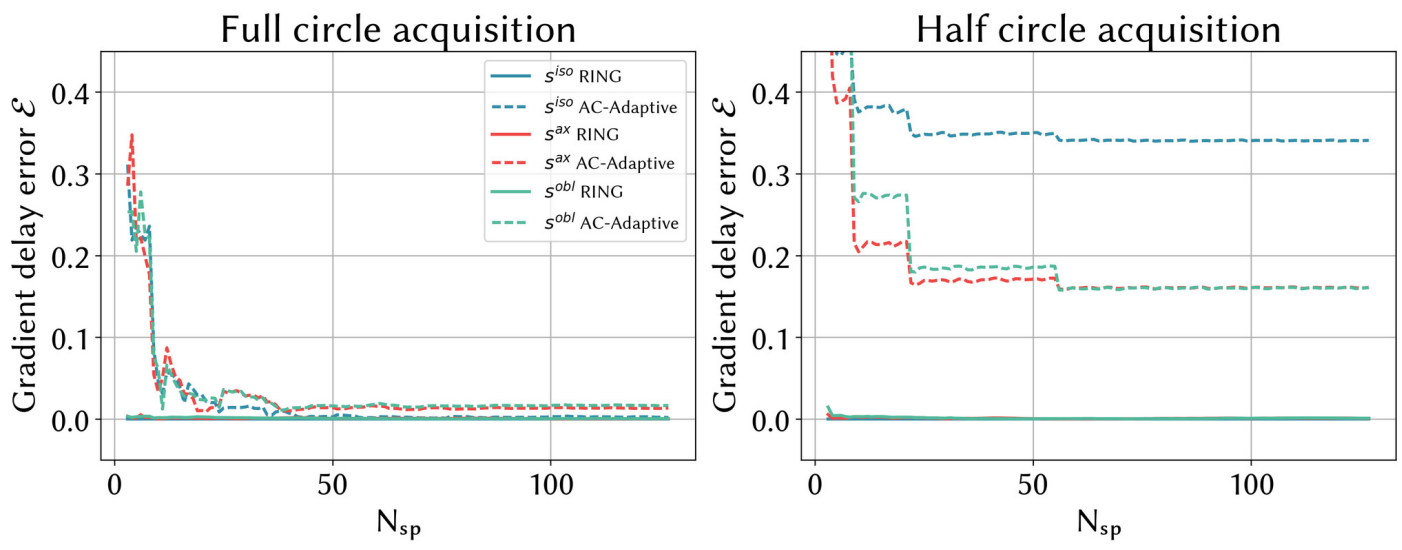

Figure 3.2.: Gradient delay error $\mathcal{E}\left(s, s_{N_{\mathrm{sp}}}^{\text {est }}\right)$ (Equation (3.12)) against the number of spokes utilized for gradient delay estimation using the RING and the AC-Adaptive method. Numerical k-space Shepp-Logan-phantom study with different nominal kspace trajectory shifts $s^{\text {iso }}=(0.3,0.3,0), s^{\text {ax }}=(0.3,-0.1,0)$ and $s^{\text {obl }}=(0.3,-0.1,0.2)$. (Left) Full circle golden angle acquisition (projection angle $\theta_{i} \in[0,2 \pi]$ ). (Right) Half circle golden angle acquisition (projection angle $\theta_{i} \in[0, \pi]$ ).

ent trajectory shifts using the AC-Adaptive method and RING are plotted over the number of spokes used for gradient delay estimation.

The RING method provides nearly perfect estimates for all investigated delays in half circle and full circle acquisitions, even if only three spokes are employed.

In contrast, the AC-Adaptive method delivers inaccurate gradient delay estimates for half circle acquisitions even if up to $N_{\mathrm{sp}}=127$ spokes are used. For full circle acquisitions at least $N_{\mathrm{sp}} \approx 20$ spokes are necessary, to provide reasonable results. For fewer numbers of spokes the gradient delay error shows unpredictable behavior, which makes the estimates unreliable. By increasing the number of utilized spokes, the estimated delays converge to constant values. However, only the isotropic delay is estimated perfectly, whereas in the axial and oblique case a deviation from the optimal values remains.

PHANTOM MEASUREMENTS. The results of the phantom measurement and the corresponding gradient delay errors $\mathcal{E}\left(s_{159}^{\text {est }}, s_{N_{\mathrm{sp}}}^{\text {est }}\right)$ over the number of spokes used for gradient delay estimation are provided in Figure 3.3.

RING provides a good gradient delay estimation and thus, effective streaking artifact reduction even for only $N_{\mathrm{sp}}=3$ spokes. The estimate is further improved when more spokes are utilized. However, the effect on the resulting image quality is only marginal, since the image is basically streaking-free for $N_{\mathrm{sp}}=3$ spokes already. Again, the method proves to be applicable to both full circle and half circle acquisition, although visual observation reveals slightly better results for full circle acquisitions with very few utilized spokes. 
a)

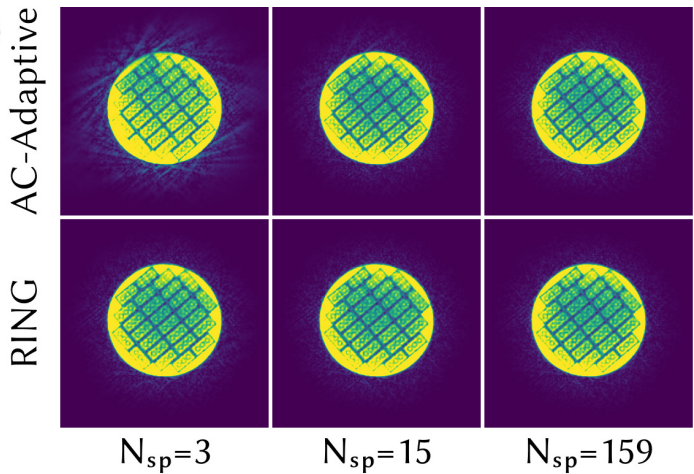

b)

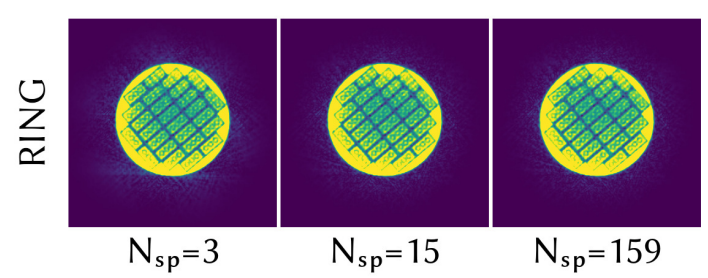

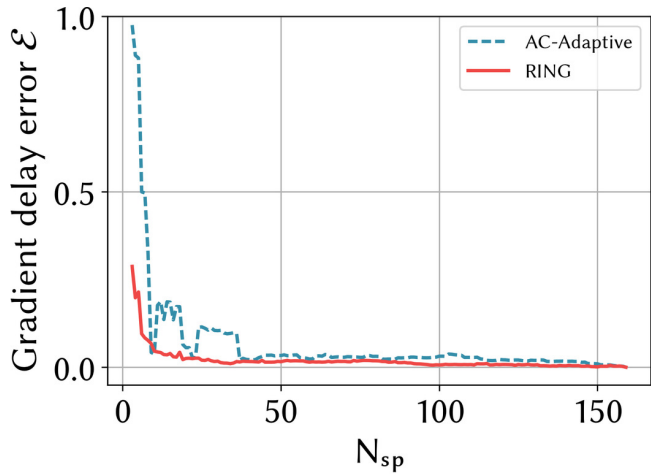

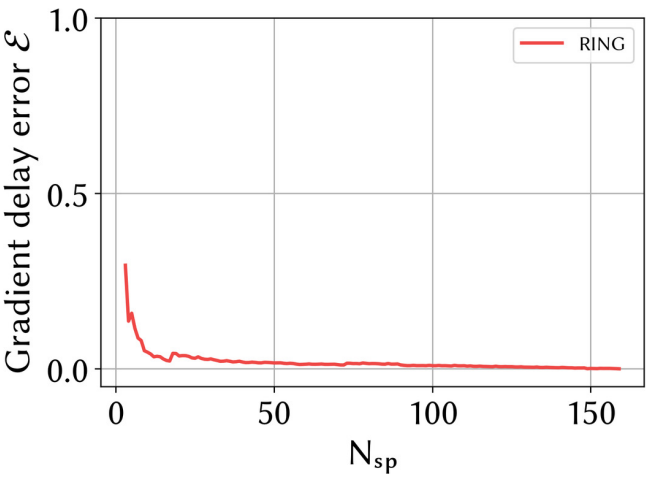

Figure 3.3.: NLINV reconstructions using 39 spokes of a brick phantom FLASH measurement with gradient delay correction estimated from $N_{\mathrm{sp}}$ number of spokes utilizing the AC-Adaptive method and the RING method. Besides, the gradient delay error $\mathcal{E}\left(s_{159}^{\text {est }}, s_{N_{\mathrm{sp}}}^{\text {est }}\right)$ (Equation (3.12)) against the number of spokes used for gradient delay estimation is depicted. (a) Full circle golden angle acquisition (projection angle $\theta_{i} \in[0,2 \pi]$ ). (b) Half circle golden angle acquisition (projection angle $\theta_{i} \in[\mathrm{o}, \pi]$ ).

The AC-Adaptive method can only be applied in the full circle case, but does not provide accurate gradient delay estimates for few numbers of spokes where the results appear worse than the uncorrected image (not shown). Although the actual convergence value is not reached until $N_{\mathrm{sp}}=37$ spokes, the results for $N_{\mathrm{sp}}=15$ already look suitable, even if some streaking artifacts can still be observed at the top.

The actual gradient delay is not known but the convergence values for full circle acquisitions, $s_{159}^{\text {AC-Adaptive }}=(0.336,0.360,-0.021)$ and $s_{159}^{\text {RING }}=(0.345,0.384,-0.009)$, are very similar for both methods. NUFFT reconstructions show an equivalent behavior and are depicted in Figure 3.6.

IN VIVO MEASUREMENTS. Figure 3.4 shows the effects of the gradient delay correction on an in vivo measurement of the human heart, as well as the gradient delay error $\mathcal{E}\left(s_{75}^{\text {est }}, s_{N_{\mathrm{sp}}}^{\text {est }}\right)$ for both the AC-Adaptive method and RING. 

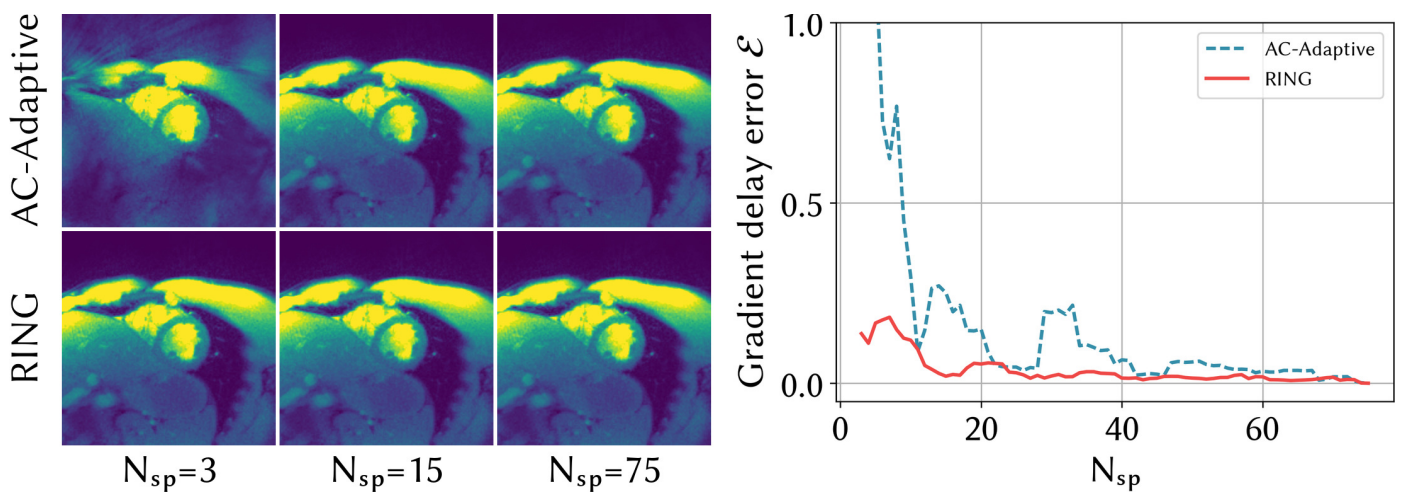

Figure 3.4.: ENLIVE reconstructions using 75 spokes of an in vivo FLASH measurement of the human heart (short-axis view, end-diastole) with gradient delay correction estimated from $N_{\mathrm{sp}}$ number of spokes utilizing the AC-Adaptive method and the RING method. Besides, the gradient delay error $\mathcal{E}\left(s_{75}^{\text {est }}, s_{N_{\text {sp }}}^{\text {est }}\right)$ (Equation (3.12)) against the number of spokes used for gradient delay estimation is depicted.

Using RING, even $N_{\mathrm{sp}}=3$ are sufficient for effective streaking artifact elimination and the gradient delay error compared to the convergence value is small for all investigated numbers of spokes.

The AC-Adaptive method also provides robust streaking suppression for $N_{\mathrm{sp}}>40$. It estimates gradient delays close to convergence value for some smaller numbers of spokes, however, these results are not reliable as the gradient delay error shows large oscillations. For very few numbers of spokes, for example, $N_{\mathrm{sp}}=3$, the estimates are useless and even amplify streaking artifacts.

The convergence values of the gradient delay estimates are $s_{75}^{\text {RING }}=(0.178,0.068$, o.068) and $s_{75}^{\text {AC-Adaptive }}=(0.263,-0.032,0.080)$ and yield comparable image quality. NUFFT reconstructions show an equivalent behavior and are depicted in Figure $3 \cdot 7$.

\subsection{DISCUSSION}

In this work we compared the widely-used AC-Adaptive method without calibration scans $[133,134,209]$ for gradient delay estimation in radial imaging with the here introduced Radial INtersection Gradient delay estimation (RING) method. The advantage of these two methods compared to other trajectory correction approaches is the simple and straightforward implementation while still being robust and accurate. All investigated experiments revealed that RING outperforms the AC-Adaptive method. It particularly possesses three advantages over the ACAdaptive method: 
First and in contrast to RING, the AC-Adaptive method needs (nearly) opposed spokes to determine the shifts via correlation analysis. However, this requirement cannot be fulfilled for half circle acquisitions, where the projection angle is distributed in the range $\theta \in[0, \pi][211]$. We demonstrated this fact in numerical simulations (Figure 3.2) for which the AC-Adaptive method provides inaccurate estimates for the gradient delays, whereas RING yields accurate results.

Second, the need for opposed spokes in the AC-Adaptive method also prohibits the use of few spokes for gradient delay estimation without calibration scans, if at the same time a uniform k-space coverage shall be guaranteed. We found this notion in all experiments Figures 3.2 to 3.4, which show pronounced streaking artifacts and gradient delay errors for few spokes using the AC-Adaptive method, while RING provides high quality results for any number of spokes.

Third, the AC-Adaptive method assumes that gradient delays solely translate into trajectory shifts in readout direction. This, however, is only the case for isotropic delays as shown in Figure 3.1. In all other cases, the trajectory additionally experiences an orthogonal shift, which in particular means that even perfectly opposed spokes do no not cover the same k-space samples. This also explains why in Figure 3.2 (left) the gradient delay error for the AC-Adaptive method only converges to zero in the isotropic case, but not in the oblique and axial case.

Recently, we have developed an extension to the AC-Adaptive method which allows gradient delay estimation from few spokes by exploiting the conjugate symmetry in k-space instead of finding opposed spokes [209], which, however, suffers from the same model inconsistency concerning orthogonal shifts.

RING requires two parameters: $N_{\text {pad }}$, which determines the amount of k-space sub-sampling to increase accuracy and $\beta$, which defines the region in which the samples of the crossing spokes are compared to find the intersection point. In preliminary investigations (not shown) we found that the accuracy of the estimates does not significantly improve for $N_{\text {pad }}>100$, so we suggest and used $N_{\text {pad }}=$ 100 in all our experiments. In general, gradient delay induced k-space shifts are $<0.51 / F O V$, thus the proposed value for $\beta=1.5$ is sufficient to find all intersection points. Note that values $\beta<1$ may result in estimation inaccuracies as spokes that are not quite orthogonal could intersect outside of the so defined region. On the other hand, we recommend to never choose $\beta \gtrsim 4$, since samples outside of the central k-space contain less energy and are more affected by noise, which might suggest a false intersection point. In the scope of this work we only considered the intersection of a spoke with its most orthogonal counterpart, which is apparently enough to yield valid results. This restriction, however, can be relaxed and the intersection of a spoke with multiple other spokes can be considered in the fit Equation (3.10), which can provide minor improvements for very few considered spokes. 
RING proofs potential as a general, lightweight on- and offline gradient delay correction tool for radial imaging. Furthermore, it can be used for AC-Adaptive frame-by-frame gradient delay correction in interactive real-time MRI. Because of its flexibility, it can be directly applied to other k-space acquisition schemes based on radials, such as radial Simultaneous Multi-Slice (SMS) [60, 62, 213] or Stack-ofStars (SOS) $[162,166]$.

\subsection{CONCLUSION}

We have presented a simple and straight forward new method dubbed RING to estimate gradient delay errors of radial trajectories from very few spokes. RING uses the gradient delay ellipse model introduced by Peters et al. [50] and Moussavi et al. [126] to fit the gradient delays Equation (3.3) using the intersection points of spokes. The method yields highly accurate and robust gradient delay estimates even for $N_{\mathrm{sp}}=3$ spokes in vivo. For its data driven, auto-calibrating nature, it can simply be inserted as a module in existing online or offline frameworks without the need to adapt the measurement protocols.

\subsection{ACKNOWLEDGEMENTS}

Supported by the DZHK (German Centre for Cardiovascular Research). Part of this research was funded by the Physics-to-Medicine Initiative Göttingen (LM der Niedersächsischen Vorab) and DFG (UE 189/1-1).

\subsection{APPENDIX: DERIVATION OF THE GRADIENT DELAY ELLIPSE MODEL}

First, we recall the definitions and results of [50]. The gradients of the logical system

$$
G_{\theta}^{\log }(t):=\left(\begin{array}{l}
G_{\text {read }}^{\log }(t) \cos \theta \\
G_{\text {read }}^{\log }(t) \sin \theta \\
G_{\text {slice }}^{\log }(t)
\end{array}\right),
$$

can be transformed into the physical system using the orthogonal transform

$$
\boldsymbol{R}:=\left(\begin{array}{lll}
R_{11} & R_{12} & R_{13} \\
R_{21} & R_{22} & R_{23} \\
R_{31} & R_{32} & R_{33}
\end{array}\right),
$$

which yields

$$
G^{\text {phy }}(t)=R G_{\theta}^{\log }(t)
$$


The timing delays $t_{x}, t_{y}$ and $t_{z}$, that effect the logical gradients, can be modeled using the delay operator

$$
\mathcal{T} G^{\text {phy }}(t)=\left(\begin{array}{c}
G_{x}^{\text {phy }}\left(t-t_{x}\right) \\
G_{y}^{\text {phy }}\left(t-t_{y}\right) \\
G_{z}^{\text {phy }}\left(t-t_{z}\right)
\end{array}\right) .
$$

Hence, the delayed gradients in the logical system can be obtained by

$$
\tilde{\boldsymbol{G}}_{\theta}^{\log }(t)=\boldsymbol{R}^{T} \mathcal{T} \boldsymbol{R} \boldsymbol{G}_{\theta}^{\log }(t),
$$

where ${ }^{T}$ denotes the transpose operation. With definition

$$
\boldsymbol{T}:=\frac{\gamma}{2 \pi}\left(\begin{array}{ccc}
t_{x} & 0 & 0 \\
0 & t_{y} & 0 \\
0 & 0 & t_{z}
\end{array}\right)
$$

the actual k-space shift is given by

$$
\begin{aligned}
& \delta \boldsymbol{k}_{\theta}=\frac{\gamma}{2 \pi} \int_{0}^{\tau}\left(\tilde{\boldsymbol{G}}_{\theta}^{\log }(t)-\boldsymbol{G}_{\theta}^{\log }(t)\right) d t \\
& \text { Eq. } \stackrel{(3.15)}{=} \frac{\gamma}{2 \pi} \boldsymbol{R}^{T} \int_{0}^{\tau}\left(\mathcal{T} G^{\text {phy }}(t)-G^{\text {phy }}(t)\right) d t \\
& \text { Eq. (3.16) } \frac{\gamma}{2 \pi} \boldsymbol{R}^{T} \int_{0}^{\tau}\left(\begin{array}{c}
G_{x}^{\text {phy }}\left(t-t_{x}\right)-G_{x}^{\text {phy }}(t) \\
G_{y}^{\text {phy }}\left(t-t_{y}\right)-G_{y}^{\text {phy }}(t) \\
G_{z}^{\text {phy }}\left(t-t_{z}\right)-G_{z}^{\text {phy }}(t)
\end{array}\right) d t \\
& \stackrel{[50]}{=} \boldsymbol{R}^{T} \boldsymbol{T}\left(\boldsymbol{G}^{\mathrm{phy}}(\tau)-\boldsymbol{G}^{\mathrm{phy}}(\mathrm{o})\right) \\
& \text { Eq. } \stackrel{(3.15)}{=} \boldsymbol{R}^{T} \boldsymbol{T} \boldsymbol{R}\left(\boldsymbol{G}_{\theta}^{\log }(\tau)-\boldsymbol{G}_{\theta}^{\log (\mathrm{o}))}\right. \\
& \text { Eq. } \stackrel{\text { (3.13) }}{=} \boldsymbol{R}^{T} \boldsymbol{T} \boldsymbol{R}\left(\begin{array}{l}
G_{\text {read }}^{\log }(\tau) \cos \theta \\
G_{\text {read }}^{\log }(\tau) \sin \theta \\
-G_{\text {slice }}^{\log }(0)
\end{array}\right) \text {. }
\end{aligned}
$$

For Equation (3.22), we assumed that the temporal delays are small compared to the flattop time of the gradients. In Equation (3.24), we used the fact that at the temporal center of the RF pulse $(t=0)$, only the slice selection gradient is active and at the center of readout $(t=\tau)$ only the readout gradients are active. We refer the reader to [50] for more details.

In general, we only have information about the projection direction Equation (3.2) $\hat{\boldsymbol{n}}_{\theta}^{\log }=(\cos \theta, \sin \theta, \mathrm{o})^{T}$ and not about the actual gradient strength of a measurement. Therefore, we separate the projection direction using

$$
\underline{G}^{\log }:=\left(\begin{array}{ccc}
G_{\text {read }}^{\log }(\tau) & 0 & 0 \\
0 & G_{\text {read }}^{\log (\tau)} & 0 \\
0 & 0 & -G_{\text {slice }}^{\log }(0)
\end{array}\right),
$$


and obtain

$$
\delta \boldsymbol{k}_{\theta}=\boldsymbol{R}^{T} \boldsymbol{T} \boldsymbol{R} \underline{G}^{\log }\left(\hat{\boldsymbol{e}}_{z}^{\log }+\hat{\boldsymbol{n}}_{\theta}^{\log }\right)
$$

with $\hat{e}_{z}^{\log }=(0,0,1)^{T}$.

The first term of the right-hand-side of Equation (3.26) is independent of the angle and thus does not effect the ellipse fit of RING and corresponds to a constant k-space offset, i.e. a linear phase in image space, and can therefore be neglected. Then, Equation (3.26) written out is given by

$$
\begin{aligned}
& \delta \boldsymbol{k}_{\theta} \approx \boldsymbol{R}^{T} \boldsymbol{T} \boldsymbol{R} \underline{\boldsymbol{G}}^{\log } \hat{\boldsymbol{n}}_{\theta}^{\log } \\
& =\left(\begin{array}{lll}
G_{\text {read }}^{\log }\left(t_{x} R_{11}^{2}+t_{y} R_{21}^{2}+t_{z} R_{31}^{2}\right) & G_{\text {read }}^{\log }\left(t_{x} R_{11} R_{12}+t_{y} R_{21} R_{22}+t_{z} R_{31} R_{32}\right) & G_{\text {slice }}^{\log }\left(t_{x} R_{11} R_{13}+t_{y} R_{21} R_{23}+t_{z} R_{31} R_{33}\right) \\
G_{\text {read }}^{\log }\left(t_{x} R_{12} R_{11}+t_{y} R_{22} R_{21}+t_{z} R_{32} R_{31}\right) & G_{\text {read }}^{\text {log }}\left(t_{x} R_{12}^{2}+t_{y} R_{22}^{2}+t_{z} R_{32}^{2}\right) & G_{\text {slice }}^{\log }\left(t_{x} R_{12} R_{13}+t_{y} R_{22} R_{23}+t_{z} R_{32} R_{33}\right) \\
G_{\text {read }}^{\text {og }}\left(t_{x} R_{13} R_{11}+t_{y} R_{23} R_{21}+t_{z} R_{33} R_{31}\right) & G_{\text {read }}^{\text {log }}\left(t_{x} R_{12} R_{13}+t_{y} R_{22} R_{23}+t_{z} R_{33} R_{32}\right) & G_{\text {slice }}^{\log }\left(t_{x} R_{13}^{2}+t_{y} R_{23}^{2}+t_{z} R_{33}^{2}\right)
\end{array}\right) \\
& \cdot\left(\begin{array}{c}
\cos \theta \\
\sin \theta \\
0
\end{array}\right) \text {. }
\end{aligned}
$$

Here, we are only interested in the in-plane gradient delays, for which it suffices to consider the top left $2 \times 2$ submatrix. By substitution we yield Equation (3.1)

$$
\begin{aligned}
\delta \boldsymbol{k}_{\theta} & \approx\left(\begin{array}{ll}
G_{\text {read }}^{\log }\left(t_{x} R_{11}^{2}+t_{y} R_{21}^{2}+t_{z} R_{31}^{2}\right) & G_{\text {read }}^{\log }\left(t_{x} R_{11} R_{12}+t_{y} R_{21} R_{22}+t_{z} R_{31} R_{32}\right) \\
G_{\text {read }}^{\text {log }}\left(t_{x} R_{12} R_{11}+t_{y} R_{22} R_{21}+t_{z} R_{32} R_{31}\right) & G_{\text {read }}^{\text {og }}\left(t_{x} R_{12}^{2}+t_{y} R_{22}^{2}+t_{z} R_{32}^{2}\right)
\end{array}\right) \hat{\boldsymbol{n}}_{\theta}^{\log } \\
& :=\left(\begin{array}{cc}
S_{x} & S_{x y} \\
S_{x y} & S_{y}
\end{array}\right) \hat{\boldsymbol{n}}_{\theta}^{\log }
\end{aligned}
$$

\subsection{SUPPLEMENTARY MATERIAL}

\subsubsection{Accuracy of the Intersection Point Determination}

We perform phantom simulations with different numerical phantoms, Signal-toNoise Ratios (SNRs) and numbers of coils to analyze the accuracy of the intersection point determination using a pixel-wise comparison of the spokes' samples.

We utilize a Shepp-Logan phantom and two other geometric phantoms. The left side of Figure 3.5 shows NUFFT reconstructions of the phantoms for different SNRs. We simulate the corresponding delayed radial k-space data $(s:=$ (0.3,-0.1, 0.2), full circle golden angle trajectory, 160 read-out samples, 159 spokes) for $c=1, \ldots, 8$ coils and add Gaussian white noise. As a measure for the noise we divide the energy of the noise-free k-space by the energy of the added noise: $\left.\mathrm{SNR}_{\mathrm{k}}=E_{s} / E_{n}\right)$. We compare all actual intersection points with the estimated intersection point using Equations (3.4) and (3.5) and $s$. For the estimation, the values for $a_{\theta_{i}}$ are determined by pixelwise comparison of the spokes' samples as described 
in the manuscript. The analytical expression used to determine the actual intersection point can be derived using Equation (3.5):

$$
a_{\theta_{i}}=\left(\frac{\hat{\boldsymbol{n}}_{\theta_{j, 2}}}{\hat{\boldsymbol{n}}_{\theta_{i, 2}} \hat{\boldsymbol{n}}_{\theta_{j, 1}}}-\hat{\boldsymbol{n}}_{\theta_{i, 1}}\right)\left(S_{x} \xi_{1}+S_{x y} \xi_{2}-\frac{\hat{\boldsymbol{n}}_{\theta_{j, 1}}}{\hat{\boldsymbol{n}}_{\theta_{j, 2}}}\left(S_{x y} \xi_{1}+S_{y} \xi_{2}\right)\right) .
$$

The central column of Figure 3.5 shows the Root-Mean-Square (RMS) error between all measured and actual intersection points for different noise levels and numbers of coils.

Furthermore, we estimate the gradient delays $s_{\mathrm{SNR}_{\mathrm{k}}, c}^{\mathrm{est}}$ for the same numbers of coils $c$ and SNRs. The gradient delay error $\mathcal{E}\left(s, s_{\mathrm{SNR}_{\mathrm{k}}, c}^{\text {est }}\right)$ (Equation (3.12)) is depicted in Figure 3.5, right column.

Both the gradient delay error and the RMS error of the intersection points improve with the number of utilized coils. This behavior is sensible as samples from all channels can be used to determine the intersection points and thus more robust results can be obtained. While a single coil yields bad results for any noise level, the use of multiple coils, which today is clinical standard, yield accurate results even for low $\mathrm{SNR}_{\mathrm{k}}$ values. The accuracy is comparable for all investigated phantoms. 

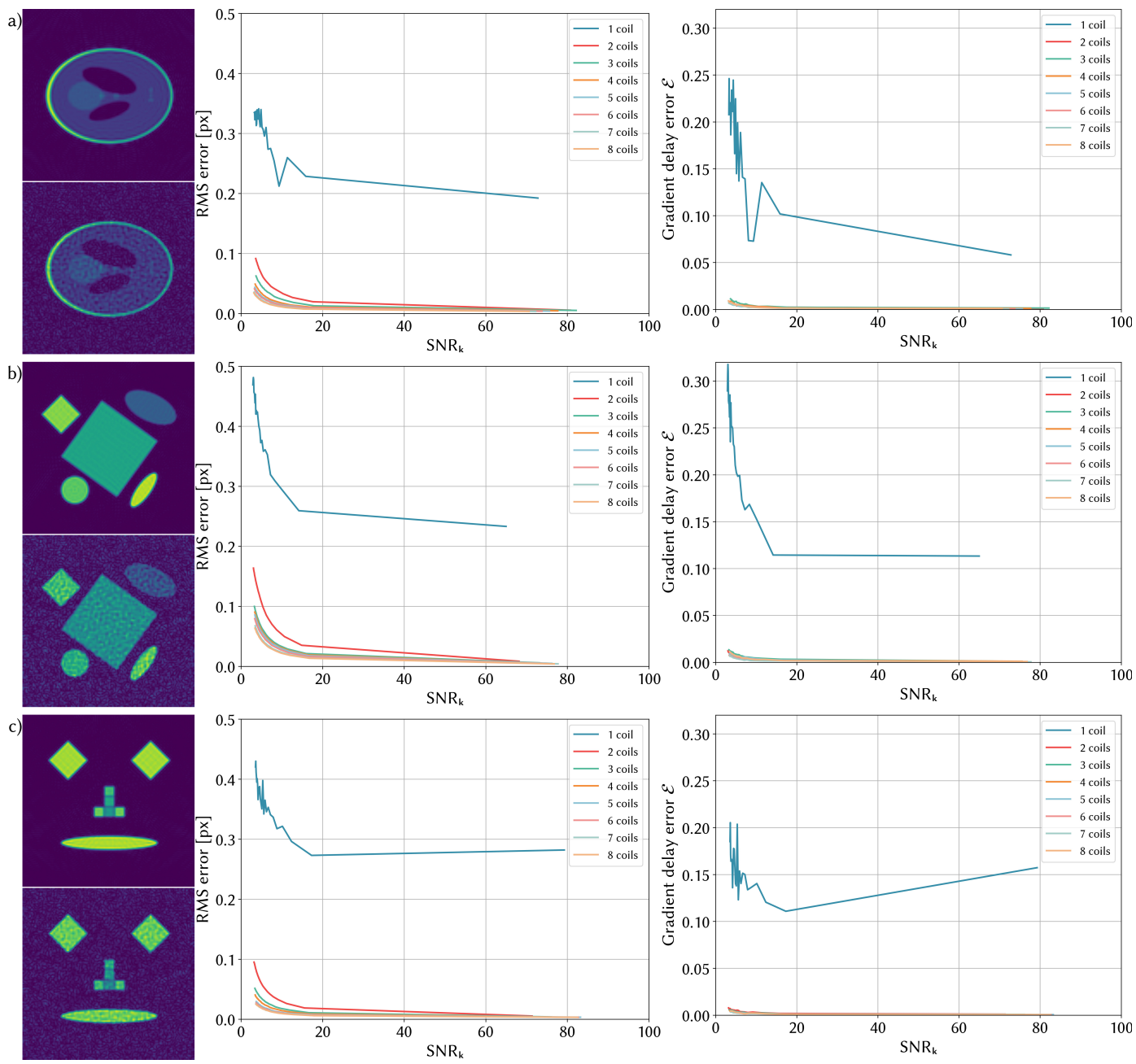

Figure 3.5.: Intersection point accuracy analysis for three different numerical phantoms (a), (b), (c). (Left) NUFFT reconstructions without noise and with $\mathrm{SNR}_{\mathrm{k}} \approx 7$. (Center) Root-mean-square error of the measured intersection point against the $\mathrm{SNR}_{\mathrm{k}}$ for different numbers of coils. (Right) Gradient delay estimation error against the $\mathrm{SNR}_{\mathrm{k}}$ for different numbers of coils. 


\subsubsection{NUFFT Reconstructions}

a)
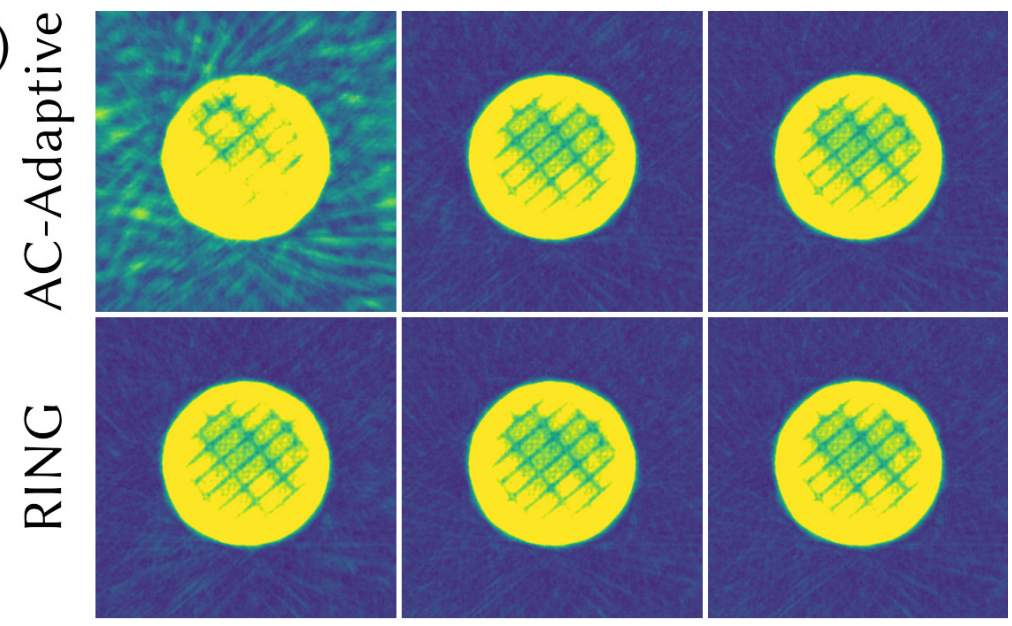

$$
N_{s p}=3
$$

$\mathrm{N}_{\mathrm{sp}}=15$

$\mathrm{N}_{\mathrm{sp}}=159$

b)
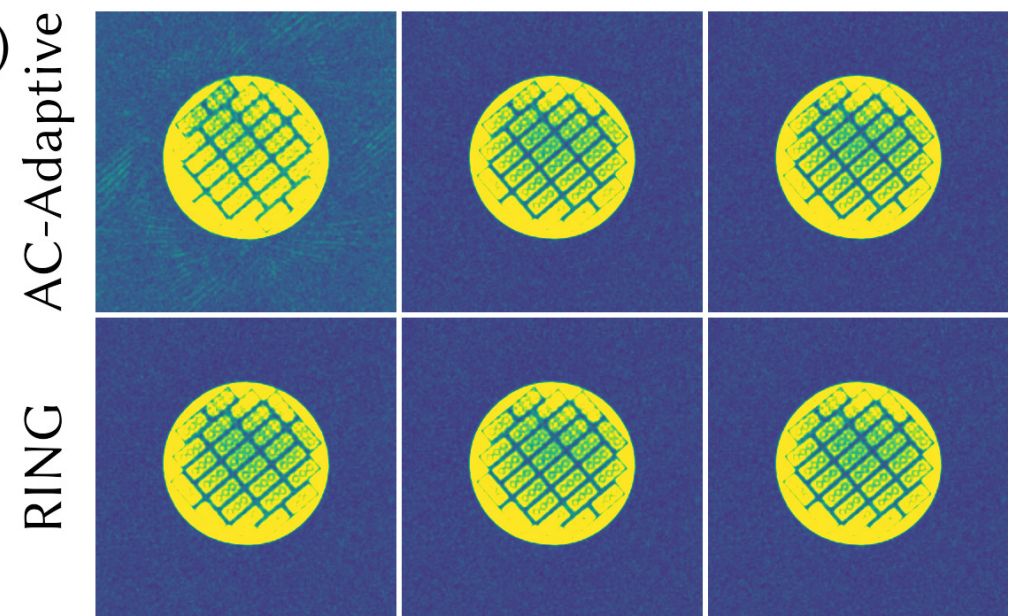

$$
N_{s p}=3
$$

$\mathrm{N}_{\mathrm{sp}}=15$

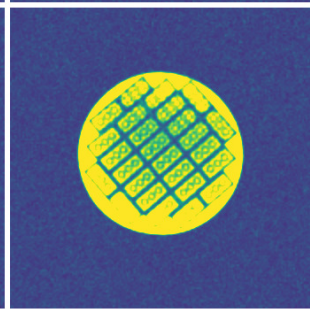

$\mathrm{N}_{\mathrm{sp}}=159$

Figure 3.6.: NUFFT reconstructions of a brick phantom FLASH measurement with gradient delay correction estimated from $N_{\text {sp }}$ number of spokes utilizing the AC-Adaptive method and the RING method. (a) 39 spokes used for reconstruction. (b) 159 spokes used for reconstruction. 


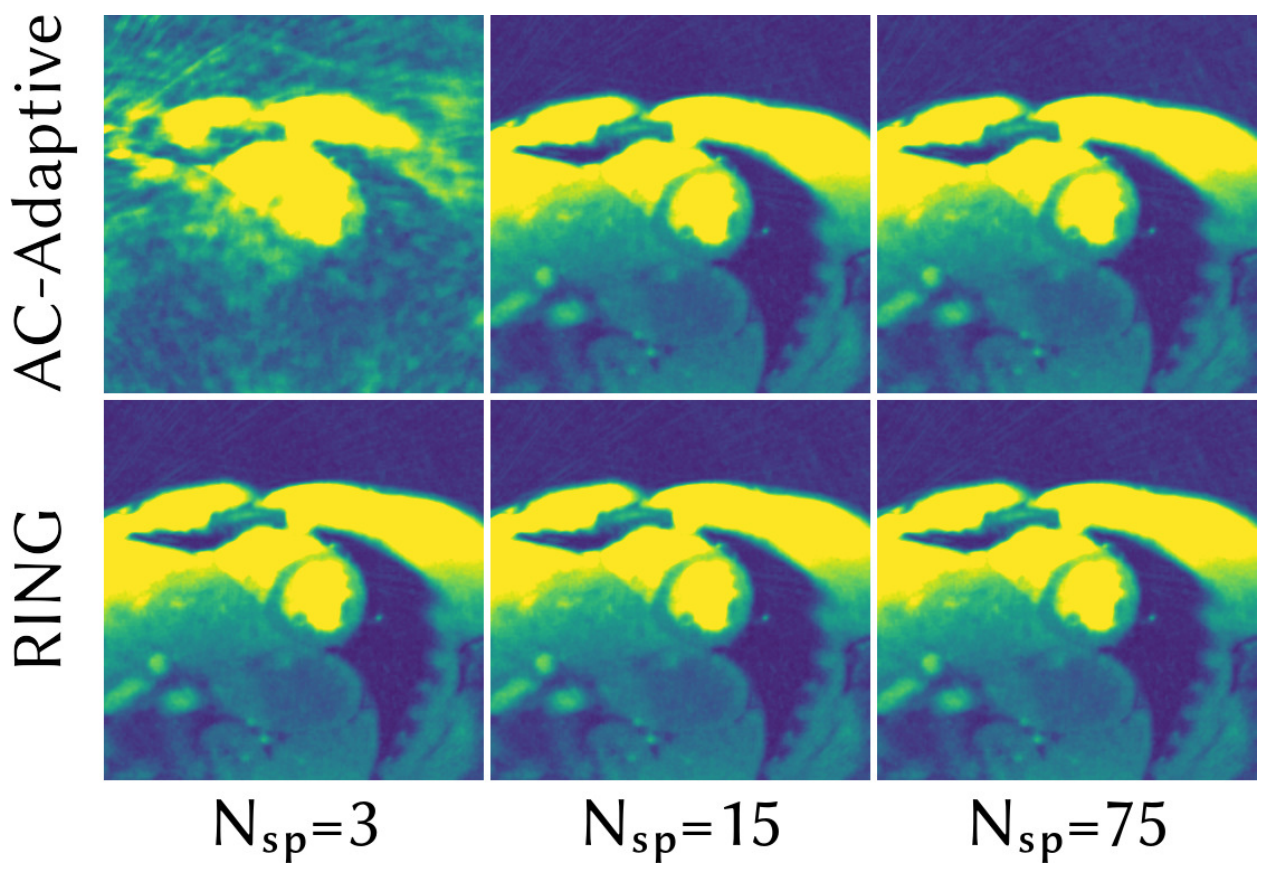

Figure 3.7.: NUFFT reconstructions using 75 spokes of an in vivo FLASH measurement of the human heart (short-axis view, end-diastole) with gradient delay correction estimated from $N_{\mathrm{sp}}$ number of spokes utilizing the AC-Adaptive method and the RING method. 

Motivated by the accurate gradient delay estimations from only few spokes demonstrated in the previous chapter, we will here use RING to estimate and correct the gradient delays frame-by-frame for an interactive real-time MRI measurement.

Parts of this work have been published as an abstract at the International Society for Magnetic Resonance in Medicine (ISMRM) 27th Annual Meeting and Exhibition in 2019 [59].

\subsection{METHODS}

We extracted 30 seconds from a random RF spoiled [90] radial FLASH measurement of a human heart with interactive changes to the slice position and orientation, which has been acquired in the scope of a different study at the University Medical Center Göttingen. The utilized acquisition parameters were TE/TR = 1.3/2.0 ms, FOV $=256 \times 256 \mathrm{~mm}^{2}$, flip angle $8^{\circ}$, base resolution 128 . The acquired $\mathrm{k}$ space was composed of 21 evenly distributed spokes per frame with rotated spoke patterns to generate five different radial trajectories, as shown in Figure 2.14a (bottom row). We estimated the gradient delays for each frame individually using RING and utilized real-time NLINV for offline reconstruction of the frame-by-frame corrected data. As a reference, we compared the results to a reconstruction for which the gradient delays estimated for the first frame were used to correct all subsequent frames. Note that the estimation of gradient delays in a pre-processing step using initial frames or prep-scans, and their use to correct all subsequent frames of the scan, is a common strategy.

\subsection{RESULTS}

Figure 4.1a depicts the temporal evolution of the gradient delay parameters $S_{x}, S_{y}$ and $S_{x y}$, introduced in Equation (3.3). The values remain approximately constant for the first 177 frames, which is in compliance with the corresponding steady slice position. Starting with frame 178 , the gradient delay estimates change distinctly as the imaging plane is subject to shifts and rotations. Moreover, slight oscillations with a period of five frames can be noticed for each parameter.

Figure 4.1b shows reconstructions for frames 100, 180, 600 and 800, which are highlighted by the dashed grey lines in Figure 4.1a. The top row shows reference 
images, for which the gradient delay estimates of the first frame were utilized for the correction of all subsequent frames. The image of frame 100 is basically free of gradient delay induced artifacts. The streakings in all other depicted frames of the reference imply imperfect gradient delay compensation. In contrast, streaking artifacts are significantly reduced when the gradient delays are estimated frameby-frame as shown in the bottom row of Figure 4.1 b.

a)

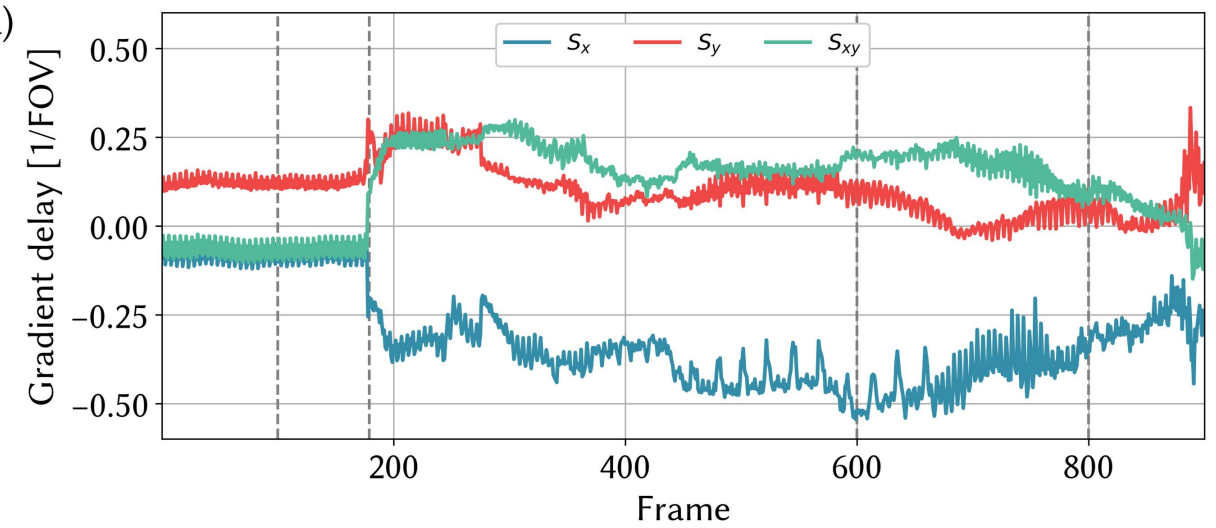

b)

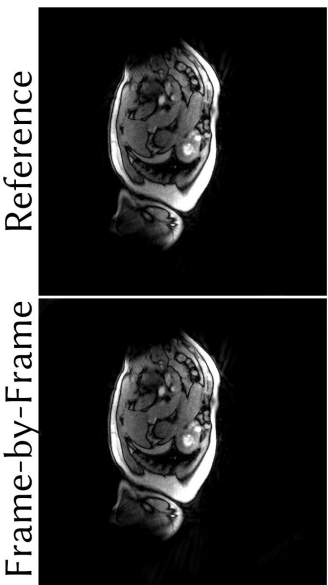

Frame $=100$

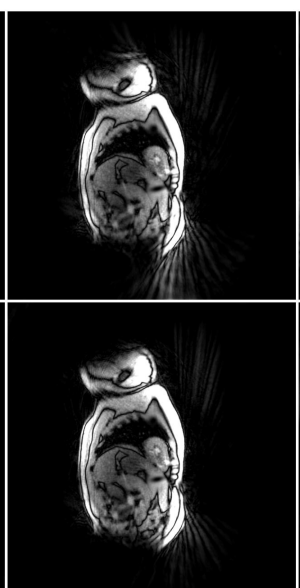

Frame $=180$

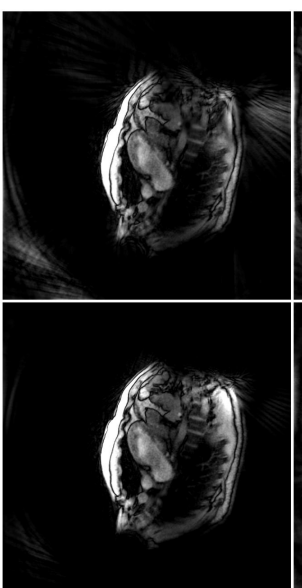

Frame $=600$

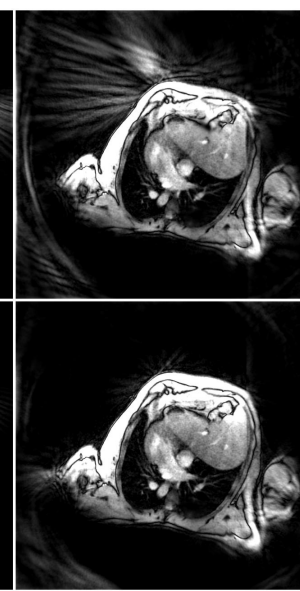

Frame $=800$

Figure 4.1.: Gradient delay correction using RING for interactive real-time MRI. (a) Evolution of the gradient delay parameters $S_{x}, S_{y}$ and $S_{x y}$ with time. For the first 177 frames the slice position and orientation is kept constant. Then, the gradient delays change because the imaging location interactively gets modified. The vertical dashed lines show the frames corresponding to the images shown in (b). (b) Comparison of real-time NLINV reconstructions with gradient delay estimation using the first frame only (top row) and the proposed frame-by-frame updated gradient delay estimations (bottom row). 


\section{$4 \cdot 3$ DIsCUSSION}

In this proof of principle study, we have successfully applied RING to an interactive real-time MRI acquisition and could reliably diminish the streaking artifacts on a frame-by-frame basis for arbitrary imaging locations and orientations.

The experiment confirms the assumption that gradient delays remain constant if the imaging plane is not changed. Accordingly, the reference image of frame 100 does not exhibit streaking artifacts, as its imaging plane corresponds to the one of the first frame, whose gradient delay estimates were used to correct all reference frames. The variations of the gradient delay parameters after frame 177 reflect the modifications to the imaging plane. The superposed oscillations with period length 5 corresponds to the 5 different utilized spoke patterns, which suggests a slight dependency of the gradient delay estimation on the used trajectory. This relation could result from uncorrected $B_{0}$ eddy currents [126], see Section 2.3.3. Nevertheless, these oscillations did not reveal a notable impact on the quality of streaking suppression.

The presented frame-by-frame gradient delay estimation using RING was performed offline. Still, we are confident that the RING algorithm can be fast enough to run online directly on the scanner. In particular, to speed up the computation the oversampling factor $N_{\text {pad }}$ could be diminished or the number of spokes to estimate the gradient delays, which does not need to coincide with the number of spokes per frame, could further be reduced - without significant loss of accuracy, as was demonstrated e.g. in Figure 3.4. Further investigations to guarantee a time-lag free online gradient delay estimation and the incorporation of the RING algorithm into the online reconstruction pipeline remain to be done in the future.

\subsection{CONCLUSION}

RING is a promising tool for the repeated determination of gradient delays in radial MRI. This study demonstrates the successful application of RING to interactive real-time MRI, which demands a frame-by-frame update of gradient delay estimates from few available spokes. 

SIMULTANEOUS MULTI-SLICE MRI USING CARTESIAN AND RADIAL FLASH AND REGULARIZED NONLINEAR INVERSION: SMS-NLINV

The following is a reprint of the original article

S. Rosenzweig, H. C. M. Holme, R. N. Wilke, D. Voit, J. Frahm, and M. Uecker. "Simultaneous multi-slice MRI using cartesian and radial FLASH and regularized nonlinear inversion: SMS-NLINV". Magn. Reson. Med. 79 (2018), pp. 2057-2066.

(C) 2017 International Society for Magnetic Resonance in Medicine. Reprinted, with permission from [6o].

$S R$ implemented the SMS sequence with contributions by $R W$ and $D V$. SR implemented the SMS extension to NLINV with the assistance of $M U$ and $C H$. SR designed and conducted all experiments and performed the analysis with the help of $M U$. JF and $M U$ supervised the analysis of the experiments. $S R$ and $M U$ wrote the manuscript with input from all authors.

The layout, the reference numbering, the orthography and the use of acronyms have been adapted to preserve the natural text flow of this thesis. No substantive changes to the content have been realized.

The technique of SMS-NLINV was described in [63] for Cartesian imaging. This reprinted article presents the basic functionality of SMS-NLINV, extends it to radial imaging, comprises a comparison to the alternative linear reconstruction approach ESPIRiT and evaluates different Cartesian and radial sampling patterns for SMS imaging. 


\section{ABSTRACT}

PURPOSE: The development of a calibrationless parallel imaging method for accelerated Simultaneous Multi-Slice (SMS) MRI based on Regularized Nonlinear Inversion (NLINV), evaluated using Cartesian and radial Fast LowAngle Shot FLASH.

THEORY AND METHODS: NLINV is a parallel imaging method that jointly estimates image content and coil sensitivities using a Newton-type method with regularization. Here, NLINV is extended to SMS-NLINV for reconstruction and separation of all simultaneously acquired slices. The performance of the extended method is evaluated for different sampling schemes using phantom and in vivo experiments based on Cartesian and radial SMS-FLASH sequences.

Results: The basic algorithm was validated in Cartesian experiments by comparison with ESPIRiT. For Cartesian and radial sampling, improved results are demonstrated compared to single-slice experiments, and it is further shown that sampling schemes using complementary samples outperform schemes with the same samples in each partition.

ConCLusion: The extension of the NLINV algorithm for SMS data was implemented and successfully demonstrated in combination with a Cartesian and radial SMS-FLASH sequence. 


\subsection{INTRODUCTION}

Accelerating image acquisition is of great importance in clinical Magnetic Resonance Imaging (MRI). Parallel imaging exploits receive-coil arrays for acceleration. Conventional reconstruction methods for parallel imaging consist of a calibration from reference lines followed by linear reconstruction [93, 94, 96, 214]. In contrast, Regularized Nonlinear Inversion (NLINV) [40] does not require a calibration step but simultaneously computes image content and coil sensitivities from all available data. Because NLINV does not depend on the presence of explicit (Cartesian) calibration data, it is ideally suited for non-Cartesian parallel imaging. For example, NLINV is used in a highly accelerated real-time MRI method based on radial sampling [42].

Many applications require the acquisition of several slices. Simultaneous MultiSlice (SMS) MRI [148] allows for significant scan time reductions and improved image quality [149, 150]. In SMS MRI several slices are excited at the same time and the resulting superposition is disentangled using special encoding schemes $[151,215]$ and/or the spatial encoding information inherent in receiver coil arrays [148]. The main benefit of accelerated SMS MRI over conventional single-slice imaging is the possibility to distribute undersampling among an additional dimension and exploit sensitivity encoding in all three dimensions, which allows for higher acceleration factors $[152,153,213,216-218]$.

The aim of this work is to extend NLINV for the reconstruction of SMS data. First, the extension of the algorithm for Cartesian and radial sampling with arbitray encoding in slice direction is introduced. For Cartesian data from an SMS-Fast Low-Angle Shot (FLASH) sequence, SMS-NLINV is compared to ESPIRiT [96]. For Cartesian and radial data, a single-slice measurement is compared to SMS acquisitions with equivalent or complementary samples in each partition. Accelerated SMS measurements of a human brain and a human heart are performed to show feasibility of in vivo scans.

\subsection{THEORY}

Table 5.1 shows the notation used in this work.

\subsubsection{SMS Encoding and Excitation Pulses}

In SMS MRI, $M$ partitions $p=1, \ldots, M$ are measured to get information about $M$ parallel slices $q=1, \ldots, M$. Please note that a fully sampled acquisition with $M$ partitions has $M$ times the number of samples compared to a single-slice experiment, and the acceleration factor of an SMS experiment is then given by $R=N^{\text {full }} / N^{\text {red }}$, with $N^{\text {full/red }}$ the number of samples acquired in a full and undersampled partition measurement, respectively. Contrary to conventional multi-slice, in each partition 
measurement all $M$ slices are excited simultaneously, i.e. superposed data are acquired. In the limit of small flip angles, an SMS radio-frequency (RF) excitation pulse, which excites $M$ slices at positions $z_{q}$ and thickness $\Delta z_{q}$, can be created by superposing conventional single-slice excitation pulses $B_{\mathrm{rf}}^{(1)}\left(z_{q}, \Delta z_{q}\right)$. To generate differently encoded partitions a unitary $M \times M$ encoding matrix $\Xi$ is included. The SMS RF excitation pulse for partition measurement $p$ is then given by

$$
\tilde{B}_{\mathrm{rf}, p}^{(M)}\left(z_{1}, \ldots, z_{M}, \Delta z_{1}, \ldots, \Delta z_{M}\right):=\sum_{q=1}^{M} \Xi_{p q} B_{\mathrm{rf}}^{(1)}\left(z_{q}, \Delta z_{q}\right) .
$$

Let $\boldsymbol{y}_{q}:=\left(y_{q}^{1}, \ldots, y_{q}^{N}\right)$ be a vector, which contains the k-spaces $y_{q}^{j}$ of slice $q$ and coils $j=1, \ldots, N$. Then, the encoded k-space of partition $p$ is given by

$$
\tilde{\boldsymbol{y}}_{p}:=\sum_{q=1}^{M} \Xi_{p q} \boldsymbol{y}_{q} .
$$

Although the derived SMS-NLINV algorithm is completely generic, we use the discrete Fourier-matrix for encoding in the scope of this work, i.e.

$$
\Xi_{p q}=\exp \left(-2 \pi i \frac{(p-1)(q-1)}{M}\right), p, q=1, \ldots, M .
$$

\subsubsection{Image Reconstruction}

If the encoded k-spaces $\tilde{\boldsymbol{y}}_{1}, \ldots, \tilde{\boldsymbol{y}}_{M}$ determined by the $M$ partition measurements are fully sampled, the k-space of each slice can be recovered by applying the inverse of the encoding matrix

$$
\boldsymbol{y}_{q}^{\mathrm{avg}}:=\sum_{p=1}^{M} \Xi_{q p}^{-1} \tilde{\boldsymbol{y}}_{p}
$$

Note that the k-spaces $\boldsymbol{y}_{q}^{\text {avg }}$ possess an Signal-to-Noise Ratio (SNR) benefit of $\sqrt{M}$ compared to single-slice experiments due to averaging given by Equation (5.4) and because $\Xi$ is unitary. Equation (5.4) can also be applied to undersampled data if the same k-space positions are acquired for all partitions. The recovered (but still undersampled) k-spaces $\boldsymbol{y}_{q}^{\text {avg }}$ can then be processed using conventional single-slice reconstruction algorithms. This still leads to an SNR benefit, but the actual advantage of SMS - the acceleration in direction perpendicular to the slices - only comes into play when distinct samples are acquired for each partition. Then, Equation (5.4) is no longer applicable and more elaborate SMS reconstruction approaches must be applied. A novel approach to tackle this reconstruction problem is introduced in the following. 
NLINV [40] can be extended for the reconstruction of encoded SMS data [219]. In NLINV, the MRI signal equation is modeled as a nonlinear operator equation,

$$
F(X)=\tilde{Y} .
$$

$X$ is the vector to be reconstructed. It contains the image content $m_{q}(r)$ and the $N$ coil sensitivities $c_{q}^{j}(\boldsymbol{r}), j=1, \ldots, N$, for each of the $M$ slices $q$, i.e. the stacked vector $X:=\left(\boldsymbol{x}_{1}, \ldots, \boldsymbol{x}_{M}\right)^{T}$ as a concatenation of the vectors $\boldsymbol{x}_{q}:=\left(m_{q}, c_{q}^{1} \ldots, c_{q}^{N}\right)^{T}$. The vector $\tilde{Y}$ contains the encoded k-spaces for all $M$ partitions and all $N$ channels, i.e. $\tilde{Y}:=\left(\tilde{\boldsymbol{y}}_{1}, \ldots, \tilde{\boldsymbol{y}}_{M}\right)^{T}$ with $\tilde{\boldsymbol{y}}_{p}:=\left(\tilde{y}_{p}^{1}, \ldots, \tilde{y}_{p}^{N},\right)^{T}$. Then, the nonlinear mapping function $F$ is given by

$$
F: X \mapsto P \Xi\left(\begin{array}{c}
\mathcal{F}\left(m_{1} \boldsymbol{c}_{1}\right) \\
\vdots \\
\mathcal{F}\left(m_{M} \boldsymbol{c}_{M}\right)
\end{array}\right), \mathcal{F}\left(m_{q} \boldsymbol{c}_{q}\right):=\left(\begin{array}{c}
\mathcal{F}\left(m_{q} c_{q}^{1}\right) \\
\vdots \\
\mathcal{F}\left(m_{q} c_{q}^{N}\right)
\end{array}\right) .
$$

Here, $\mathcal{F}$ is the (two-dimensional) Fourier transform and $\Xi$ is an encoding matrix, e.g. Equation (5.3). The projection matrix $\boldsymbol{P}$ is defined by

$$
\boldsymbol{P}:=\left(\begin{array}{ccc}
P_{1} & & 0 \\
& \ddots & \\
0 & & P_{M}
\end{array}\right),
$$

where $P_{p}$ is the orthogonal projection onto the k-space trajectory used for partition $p=1, \ldots, M$. A more compact notation for Equation (5.6) can be given by introducing the operator $C$, which performs the multiplication of the object with the sensitivities:

$$
F: X \mapsto P \Xi \mathcal{F C X}
$$

The forward operator $F$ weights the magnetization $m_{q}$ of slice $q$ with the coil sensitivities $c_{q}=\left(c_{q}^{1}, \ldots, c_{q}^{N}\right)^{T}(C)$, transforms into k-space $(\mathcal{F})$, encodes $(\Xi)$ and samples $(\boldsymbol{P})$. The derivative $D F$ and its adjoint $D F^{H}$ will be used later to solve the inverse problem Equation (5.5) and are given in Section 5.7.2. Figure 5.1 shows a flow chart of the operators $F, D F$ and $D F^{H}$.

Equation (5.5) is highly underdetermined, hence prior knowledge has to be incorporated to prevent image content to be assigned to coil profiles and vice versa. While the image content can contain strong variations and edges, coil profiles in general are smooth functions, so a smoothness-demanding norm can be applied. Uecker et al. suggest a Sobolev norm

$$
\|f\|_{H^{l}}:=\left\|a(I-b \Delta)^{l / 2} f\right\|_{L^{2}}
$$


with $l$ a positive integer, $I$ the identity matrix, $a$ and $b$ scaling parameters and $\Delta=$ $\partial_{x}^{2}+\partial_{y}^{2}$ the two-dimensional (2D) Laplacian. Hence, in Fourier space the standard L2-norm has to be weighted by the additional term $a\left(1+b|| k \|^{2}\right)^{l / 2}$, which penalizes high spatial frequencies. This regularization is implemented by transforming $X=$ $\left(\boldsymbol{x}_{1}, \ldots, \boldsymbol{x}_{M}\right)^{T}$ using a weighting matrix $\mathcal{W}^{-1}$.

We denote $X^{\prime}:=\mathcal{W}^{-1} X$ and $\boldsymbol{x}_{q}^{\prime}:=W^{-1} \boldsymbol{x}_{q}=\left(m_{q}, c_{q}^{\prime 1}, \ldots, c_{q}^{\prime N}\right)^{T}$. This yields a transformed but equivalent system of equations

$$
G\left(X^{\prime}\right):=F \mathcal{W} X^{\prime}=\tilde{Y},
$$

which is solved using the Iteratively Regularized Gauss-Newton Method (IRGNM). As a first step, the IRGNM linearizes Equation (5.10),

$$
\tilde{Y}=\left.D G\right|_{X_{n}^{\prime}} d X^{\prime}+G X_{n}^{\prime}
$$

where $X_{n}^{\prime}$ is the estimate of the $n^{\text {th }}$ Newton step and $\left.D G\right|_{X_{n}^{\prime}}$ is the Jacobian of $G$ at $X_{n}^{\prime}$. This equation is solved in the least-squares sense and with regularization using the Conjugate Gradient (CG) algorithm. The corresponding cost function to be minimized in every Newton step is

$$
\Phi\left(d X^{\prime}\right)=\underset{d X^{\prime}}{\operatorname{argmin}}\left(\left\|\left.D G\right|_{X_{n}^{\prime}} d X^{\prime}-\left(\tilde{Y}-G X_{n}^{\prime}\right)\right\|_{L^{2}}^{2}+\beta_{n}\left\|X_{n}^{\prime}+d X^{\prime}\right\|_{L^{2}}^{2}\right),
$$

where the L2 penalty term $\beta_{n}\left\|X_{n}^{\prime}+d X^{\prime}\right\|_{L^{2}}^{2}=\beta_{n}\left\|\mathcal{W}^{-1} X_{n+1}\right\|_{L^{2}}^{2}$, with $\beta_{n}=\beta_{0} h^{n}$ and $h \in(\mathrm{O}, 1)$, implies Tikhonov regularization.

\subsubsection{Implementation for Cartesian and non-Cartesian Data}

We assume Equation (5.5) to be given in discretized form and all functions are represented by vectors of point values on a rectangular grid. For Cartesian sampling, $\mathcal{F}$ can be implemented exactly as a discrete Fourier transform, and $P_{p}$ is a diagonal matrix with ones at sample positions and zeros elsewhere. The 2D Fourier transform $\mathcal{F}$ always appears in combination with the encoding matrix $\Xi$, which in this work is the discrete Fourier-matrix Equation (5.3). Thus, $\Xi \mathcal{F}$ and its adjoint can simply be implemented as a three-dimensional Fast Fourier transform and its adjoint. Note that the $2 \mathrm{D}$ Fourier transform $\mathcal{F}$ is a discretized version of a continuous Fourier transform, whereas the Fourier-encoding $\Xi$ is discrete by definition.

For non-Cartesian sampling, $P_{p}$ projects onto arbitrary positions in k-space. As in non-Cartesian SENSE, $P \mathcal{F}$ can be implemented with a non-uniform Fourier transform [220]. The term $\mathcal{F}^{H} \Xi^{H} P \Xi \mathcal{F}$ is the main operation, which occurs in each iteration step. As described previously [121], it can be interpreted as a non-periodic convolution with a point-spread function, which has to be evaluated on a region 

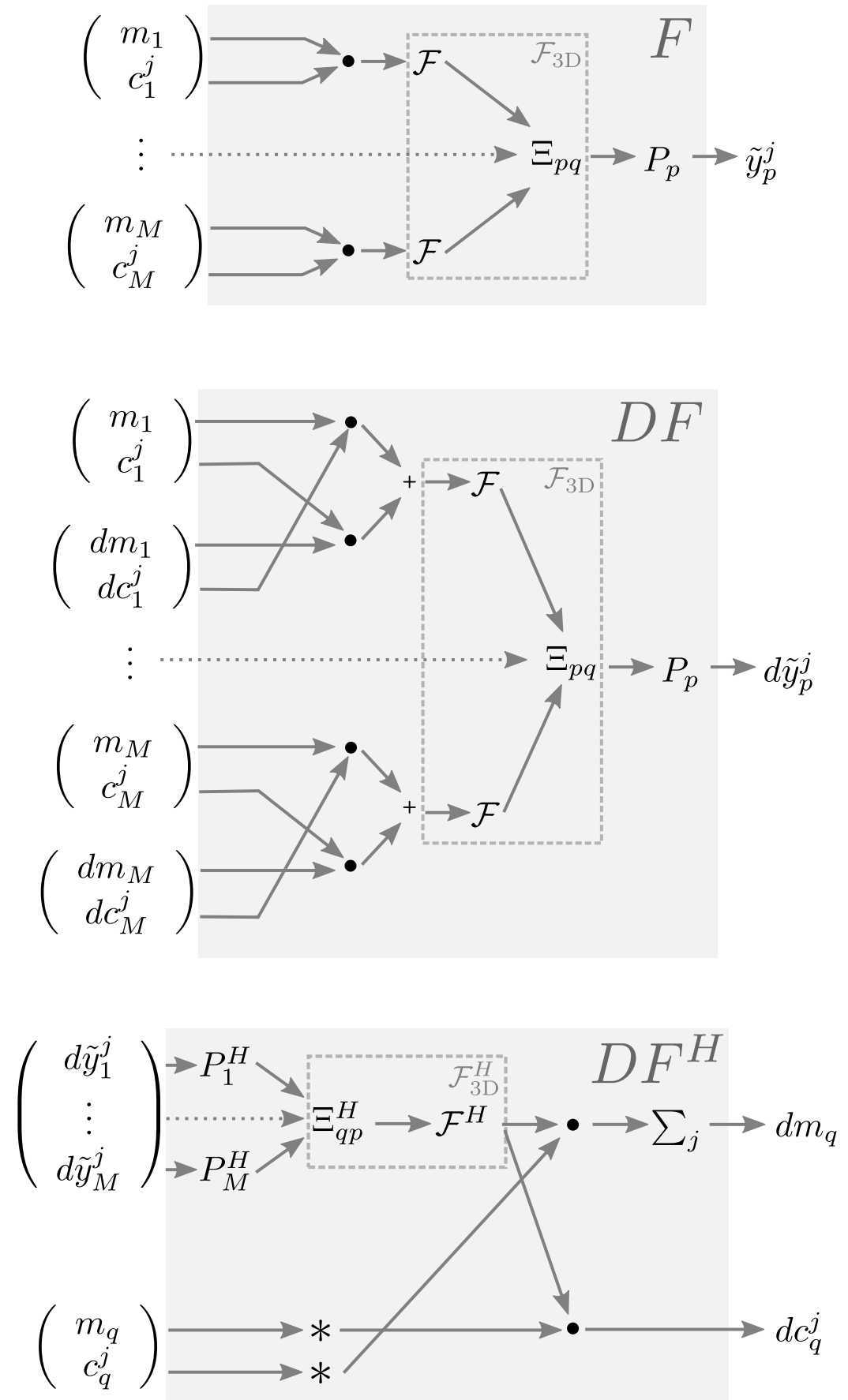

Figure 5.1.: Flow chart for the calculation of the forward operator $F$, its derivative $D F$ and the adjoint of the derivative $D F^{H}$. $\tilde{y}$ : Encoded k-space data. $m$ : Magnetization. $c$ : Coil sensitivity. $P$ : Projection onto k-space trajectory. $\mathcal{F}: 2 \mathrm{D}$ Fourier transform. $\Xi_{p q}$ : Encoding matrix. $\cdot:$ Pointwise multiplication. + : Addition. $*$ : Complex conjugation. 
with compact support defined by the Field of View (FOV). Thus, an efficient implementation is achieved with the Fast Fourier Transform (FFT) on a 2-fold enlarged grid to implement the non-periodic convolution using Toeplitz embedding. This requires only a minor modification of the Cartesian implementation, which can then be used with data gridded once onto the Cartesian grid in a preparatory step and with a pre-computed point-spread function.

\subsubsection{Sampling Schemes}

All utilized Cartesian sampling patterns possess $L_{\text {ref }}$ reference lines in the k-space center, whereas the periphery is undersampled by a factor $R$. For each of the $M$ partition measurements we can use a distinct undersampling pattern. The CAIPIRINHA technique can improve the image quality for SMS acquisitions by acquiring alternating lines between each partition $[152,221]$. Alternatively, in each partition the same samples can be acquired (aligned pattern).

In radial measurements k-space samples are acquired along spokes. Let $N_{\mathrm{sp}}$ be the total number of acquired spokes per partition. Then, the angle between consecutive spokes of a partition is set to $\alpha_{\mathrm{sp}}=2 \pi / N_{\mathrm{sp}}$, which guarantees uniform k-space coverage and prevents strong gradient delay artifacts by opposing the acquisition direction of adjacent spokes. For each partition the k-space trajectory, i.e. the spoke distribution scheme, can be chosen individually. Figure 5.2 shows three possible spoke distribution schemes: (1) The aligned scheme acquires the same spokes for each partition. (2) In the linear-turn scheme the initial spoke pattern is rotated by

$$
\alpha_{\mathrm{trn}}^{\mathrm{LIN}}=(p-1) \cdot \frac{\pi}{N_{\mathrm{sp}} \cdot M}
$$

for partition $p=1, \ldots, M$, assuring the acquisition of complementary samples and uniform spoke distribution (cf. Figure 5.2b). (3) Fourier-encoding in SMS MRI can also be seen as an additional phase-encoding in $k_{z}$ direction. The acquisition of many slices is therefore very similar to a Stack-of-Stars (SOS) sequence in true threedimensional imaging for which Zhou et al. [166] showed that a golden angle-like rotation of the spoke distribution results in a higher image quality than aligned or linearly varied distributions. Here the turn angle for partition $p$ is given by

$$
\alpha_{\mathrm{trn}}^{\mathrm{GA}}=\left((p-1) \cdot \frac{\pi}{N_{\mathrm{sp}}} \cdot \frac{\sqrt{5}-1}{2}\right) \bmod \frac{\pi}{N_{\mathrm{sp}}} .
$$

This scheme provides a more uniform local three-dimensional k-space coverage as can be seen in Figure $5.2 \mathrm{C}$, but the spokes themselves are not as evenly distributed as in the linear-turn scheme. Turn-based spoke distribution schemes in combination with Fourier-encoded partition measurements are known to improve image quality similar to CAIPIRINHA in the Cartesian case [153, 221]. 


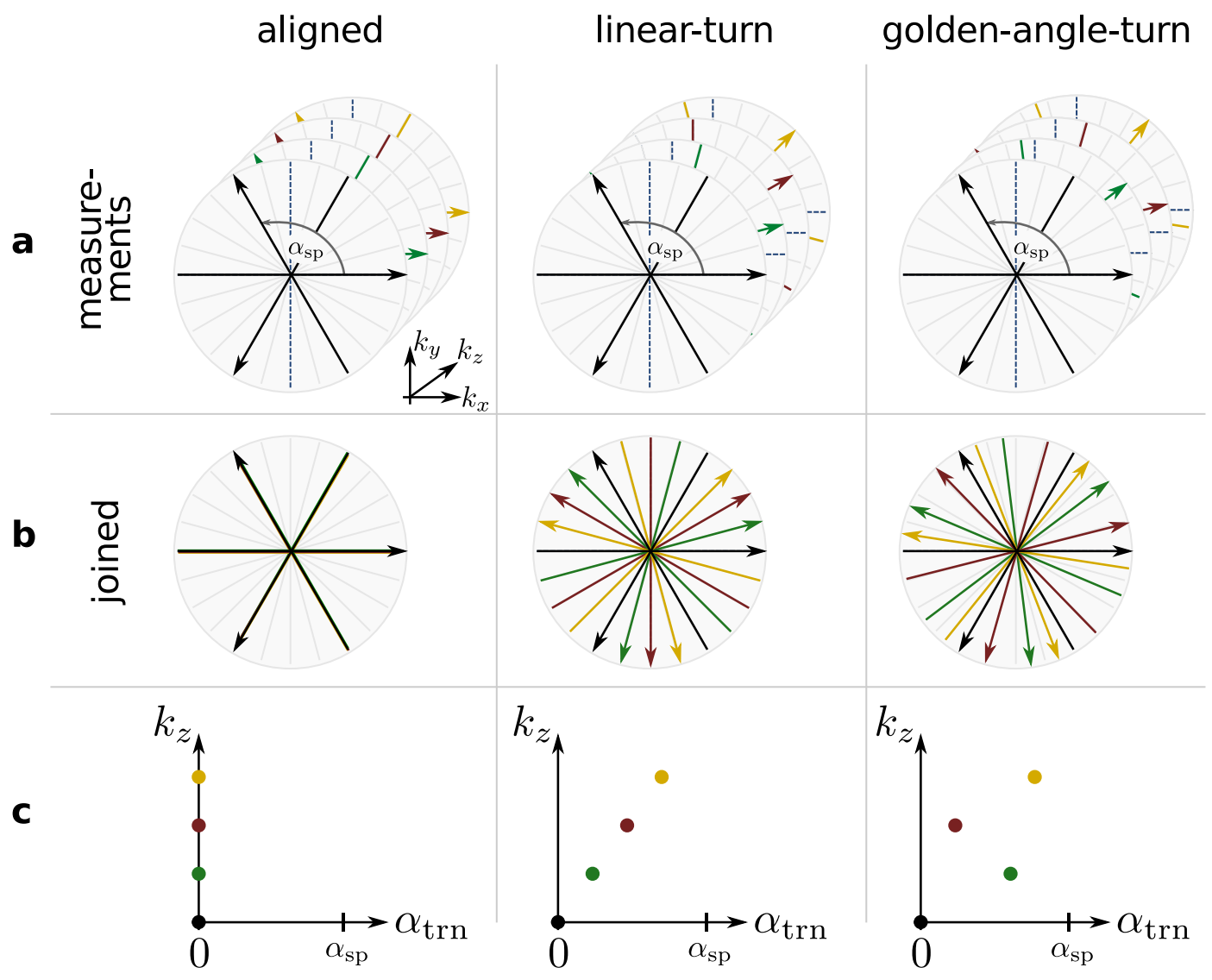

Figure 5.2.: Schematic for three radial sampling schemes (multi-band factor $M=4$ and $N_{\mathrm{sp}}=3$ spokes per partition measurement). Aligned: Same spokes acquired for each partition. Linear-turn: Linearly varied rotation angle $\alpha_{\operatorname{trn}}$ of the initial spoke distribution. Golden-angle-turn: Rotation angle $\alpha_{\text {trn }}$ chosen according to the golden angle. (a) Spoke distribution for all 4 partitions. $\alpha_{\mathrm{sp}}$ is the angle between consecutive spokes. The arrow hints the readout direction. (b) Spokes of all partitions plotted in one diagram. (c) $k_{z}$ plotted against the rotation angle $\alpha_{\text {trn }}$. 


\subsubsection{Post-Processing}

Although the matrix $\mathcal{W}$ promotes adequate distribution of image content and coil sensitivities, the results may still exhibit minor large scale intensity variations compared to a conventional Root-Sum-of-Squares (RSS) reconstruction. This can be compensated for by multiplying the image content with the RSS of the coil profiles:

$$
m_{q}^{\text {final }}=m_{q} \cdot \sqrt{\sum_{j=1}^{N}\left|c_{q}^{j}\right|^{2}}
$$

\section{$5 \cdot 3$ METHODS}

Cartesian and radial 2D FLASH sequences with adapted RF excitation pulses for Fourier-encoded SMS excitation as described in the theory section were developed and utilized in this study. All experiments were conducted on a Magnetom Skyra 3T (Siemens Healthcare GmbH, Erlangen, Germany) scanner using a 20-channel head/neck coil for phantom and human brain measurements and a combined thorax and spine coil with 26 channels for human heart measurements. All phantom measurements $\left(\mathrm{FOV}=170 \times 170 \mathrm{~mm}^{2}\right.$, matrix size $192 \times 192$, slice thickness $\Delta z=6 \mathrm{~mm}$ ) were performed on a custom-made phantom (Figure 5.3) consisting of ABS bricks (LEGO) being immersed in pure water.

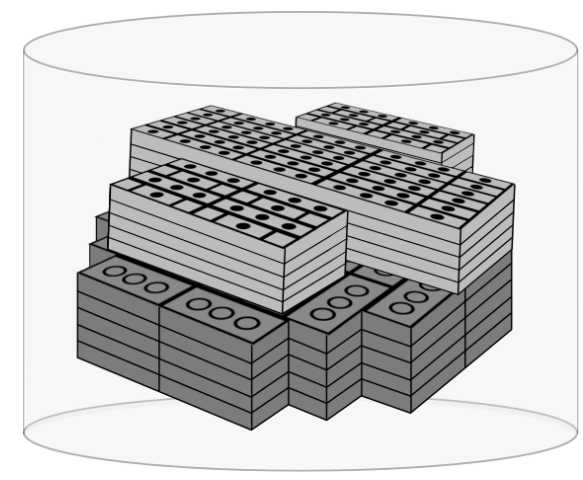

Figure 5.3.: Schematic of the custom-made phantom consisting of LEGO bricks in pure water.

It is designed such that the proton density of the top and bottom part of the phantom differ distinctly from each other. This property can be used to demonstrate the capability of SMS-NLINV to disentangle simultaneously excited slices. The in vivo brain measurements $\left(\mathrm{FOV}=230 \times 230 \mathrm{~mm}^{2}\right.$, matrix size $192 \times 192$, slice thickness $\Delta z=4 \mathrm{~mm}$, flip angle $\theta=25^{\circ}$ ) as well as the heart measurements $\left(\mathrm{FOV}=256 \times 256 \mathrm{~mm}^{2}\right.$, matrix size $160 \times 160$, slice thickness $\Delta z=6 \mathrm{~mm}$, flip angle 
$\theta=8^{\circ}$ ) were performed on volunteers with no known illnesses. In all experiments, all simultaneously acquired slices are separated by a fixed distance $d$. NLINV as well as SMS-NLINV were implemented in the C-based software package BART [210]. The initial guess was $m_{q}=1(q=1, \ldots, M)$ for the magnetizations and $c_{q}^{j}=0$ $(q=1, \ldots, M, j=1, \ldots, N)$ for the coil sensitivities. The parameters for the Sobolev norm were set to $a=1, b=220$ and $l=32$. The initial regularization parameter was $\beta_{0}=1$ with reduction factor $h=1 / 2$. In the interest of reproducible research, code and data to reproduce the experiments are made available on Github. ${ }^{1}$

To confirm the basic functionality of SMS-NLINV, a Cartesian SMS measurement with multi-band factor $M=2$ (slice distance $d=60 \mathrm{~mm}, \mathrm{TE} / \mathrm{TR}=4.4 / 8.3 \mathrm{~ms}$, flip angle $\theta=15^{\circ}$ ) was performed on the brick phantom. A full k-space was acquired and undersampling was performed retrospectively by multiplication with the corresponding patterns. The full and a retrospectively undersampled k-space (CAIPIRINHA pattern, $R=4, L_{\text {ref }}=12$ ) were reconstructed with SMS-NLINV. For comparison, reconstructions were also performed using the L2-regularized ESPIRiT algorithm [96], which is based on SENSE [94] and can therefore also be applied to SMS data [152]. To validate the accuracy of the results, difference images between the full and undersampled reconstructions were calculated. To assure proper difference images for ESPIRiT reconstructions, the complex-valued sliceimages were multiplied with the corresponding coil sensitivities followed by an RSS combination. The post-processing step in SMS-NLINV already compensates for intensity variations, thus adequate difference images can be calculated using the magnitude of the resulting images. We performed the same experiment using $L_{\text {ref }}=4$ reference lines to demonstrate the advantage of SMS-NLINV over ESPIRiT given a very small calibration region.

The CAIPIRINHA technique can significantly improve the image quality of SMS experiments $[152,221]$. We confirm these findings for SMS-NLINV by comparing retrospectively undersampled SMS measurements $(\mathrm{TE} / \mathrm{TR}=4.8 / 9.1 \mathrm{~ms}$, flip angle $\theta=15^{\circ}, L_{\text {ref }}=12, R=4$ ) with CAIPIRINHA patterns to SMS measurements with aligned patterns using the multi-band factors $M=2$ (slice distance $d=60 \mathrm{~mm}$ ) and $M=3$ (slice distance $d=30 \mathrm{~mm}$ ). The absolute slice locations were chosen such that the outermost slices in both experiments were located at the same positions, which allowed a comparison of the respective slice images. A reference measurement was performed with each investigated slice acquired separately in a single-slice experiment and reconstructed with regular NLINV using equivalent reconstruction parameters. Apart from reduced SNR, the single-slice measurements should be identical to the acquisition with the aligned patterns.

The same experiment was performed using a radial trajectory to rule out errors with the radial SMS-FLASH sequence $\left(\mathrm{TE} / \mathrm{TR}=2.0 / 3.1 \mathrm{~ms}\right.$, flip angle $\theta=15^{\circ}, N_{\mathrm{sp}}=$ 29 spokes per partition) and the SMS-NLINV reconstruction for non-Cartesian data.

1 https://github.com/mrirecon/SMS-NLINV 
Again, the improved k-space coverage of interleaved acquisitions, i.e. the use of linear-turn- and golden-angle-turn-based spoke distributions, should provide better results than aligned distributions or single-slice measurements with the same reduction factor. As a reference we performed single slice measurements on the same slices using $N_{\mathrm{sp}}=301$ spokes to achieve Nyquist sampling even in the outer region of $\mathrm{k}$-space.

Finally, we present two in vivo experiments. First, $M=5$ slices (slice distance $d=$ $60 \mathrm{~mm}, \mathrm{TE} / \mathrm{TR}=4.0 / 9.8 \mathrm{~ms}, N_{\mathrm{sp}}=39$ spokes per partition) of a human brain were acquired using the golden-angle-turn scheme. Reconstructions were performed using SMS-NLINV and L2-ESPIRiT. Calibration using ESPIRiT requires a four step procedure: (1) the reconstruction of a fully-sampled Cartesian calibration regions using gridding (for all partitions) (3) disentangling of the partitions into slices using the inverse of matrix $\Xi$, (3) Fourier transformation back into a Cartesian kspace (for each slice), and (4) actual calibration from the Cartesian k-space data (for each slice). For this procedure to work, only the region in k-space, which fulfills the Nyquist criterion in all partitions can be used. The size of the calibration region for ESPIRiT $R_{\mathrm{cal}}$ is limited by the Nyquist criterion and was calculated to be $R_{\text {cal }}=35 \times 35$. Second, $M=2$ slices (slice distance $d=40 \mathrm{~mm}, \mathrm{TE} / \mathrm{TR}=1.37 / 2.2 \mathrm{~ms}$, $N_{\mathrm{sp}}=35$ spokes per partition) of a human heart were acquired without electrocardiogram (ECG)-triggering [42].

To be able to reconstruct a single frame without temporal regularization or filtering, we combined 5 interleaves with 7 spokes per partition to obtain a single data set with 35 spokes per partition and linear-turn scheme.

\subsection{RESULTS}

\subsubsection{Cartesian Data}

Figure 5.4a shows ESPIRiT and SMS-NLINV reconstructions of a 4-fold undersampled Cartesian SMS measurement with multi-band factor $M=2$ and $L_{\text {ref }}=12$ reference lines. The SMS-NLINV algorithm can completely disentangle the superposed slices without significant artifacts after $i t=9$ Newton steps. The resulting image quality is equivalent to ESPIRiT. Figure 5.4a also depicts difference images of undersampled and full reconstructions for both methods. For better visibility the image intensity was increased by a factor of 5 . In all difference images almost no residual image content can be observed and mostly noise is present, which means that almost all aliasing artifacts could be eliminated. The enhanced noise in the central region is a consequence of the specific Cartesian sampling pattern. Figure $5.4 \mathrm{~b}$ shows the same reconstructions using a reduced calibration region. Whereas we find significant aliasing artifacts for ESPIRiT, SMS-NLINV still provides good results after $i t=10$ Newton steps. 
a 12 reference lines
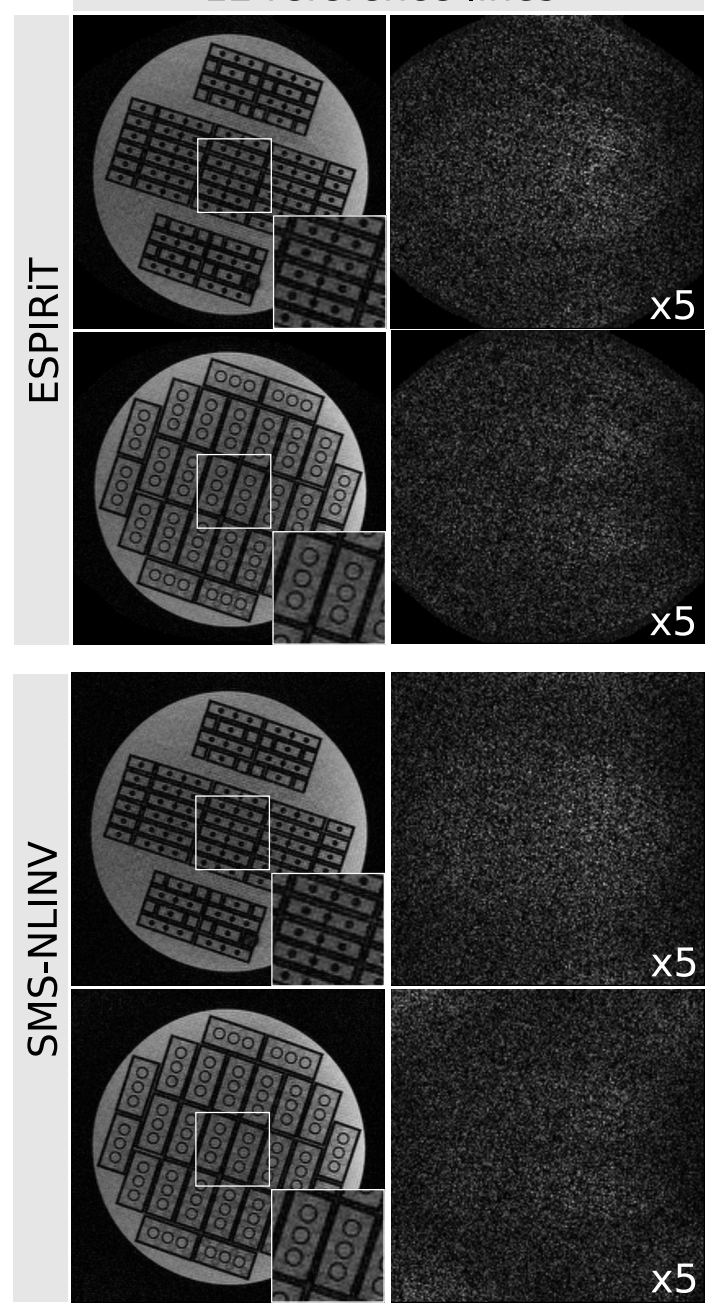

b
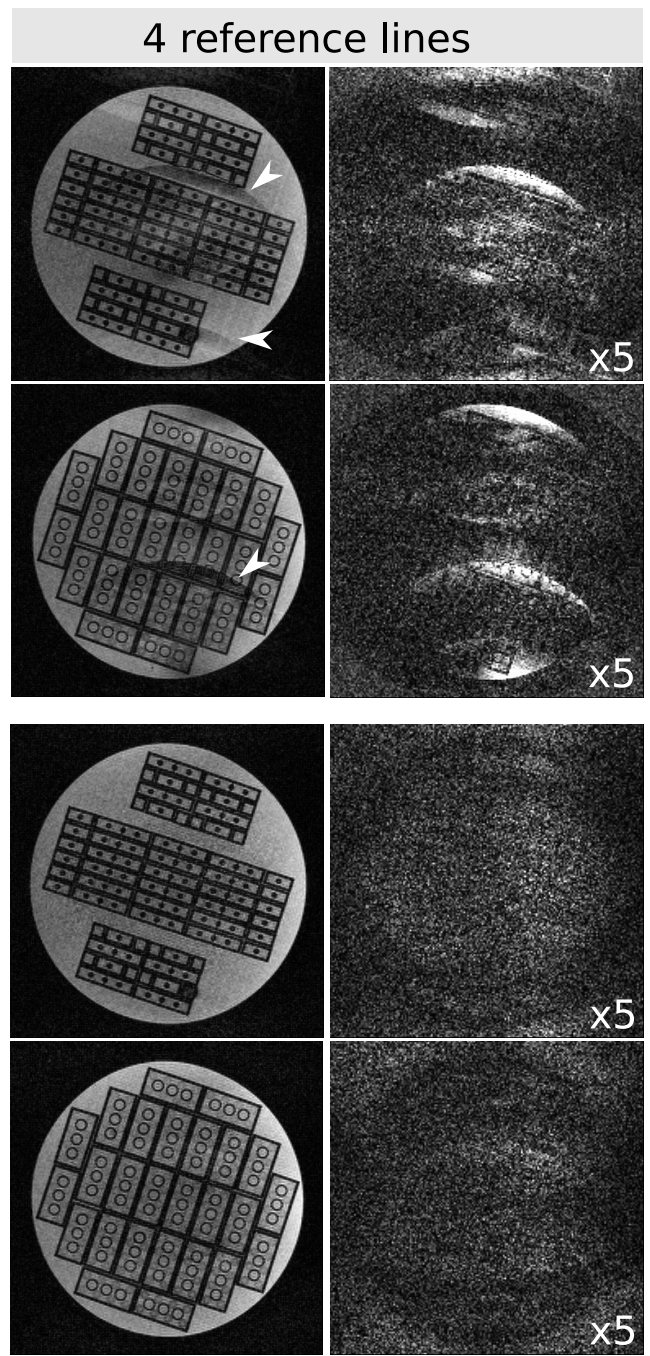

Figure 5.4.: (a) Reconstructions of a 4-fold undersampled Cartesian SMS measurement with multi-band factor $M=2$ (slice distance $d=60 \mathrm{~mm}, L_{\text {ref }}=12$ reference lines) and corresponding difference images to full reconstructions: ESPIRiT and SMS-NLINV after $i t=9$ Newton steps. A magnified Region of Interest (ROI) indicated by a white rectangle is shown as inset on the bottom right. For better visibility, the image intensity of the difference images was increased by a factor of 5. (b) Same experiment as in (a) using only $L_{\text {ref }}=4$ reference lines and $i t=10$ Newton steps for SMS-NLINV. The arrows highlight aliasing artifacts. 
Figure 5.5 shows SMS-NLINV reconstructions of Cartesian SMS acquisitions with multi-band factors $M=2$ and $M=3$ after $i t=10$ Newton steps using aligned and CAIPIRINHA patterns. As a comparison, the figure also depicts NLINV reconstructions of single-slice measurements for the same slices using it $=10 \mathrm{New}$ ton steps. For both pattern types and multi-band factors the superposition can be completely disentangled and no severe undersampling artifacts are present. However, the image quality resulting from the CAIPIRINHA data is clearly superior to aligned SMS and single-slice data. Besides an SNR benefit, the images of aligned SMS do not show any advantages compared to the single-slice images. By contrast, the CAIPIRINHA images resolve small details of the phantom bricks much better.
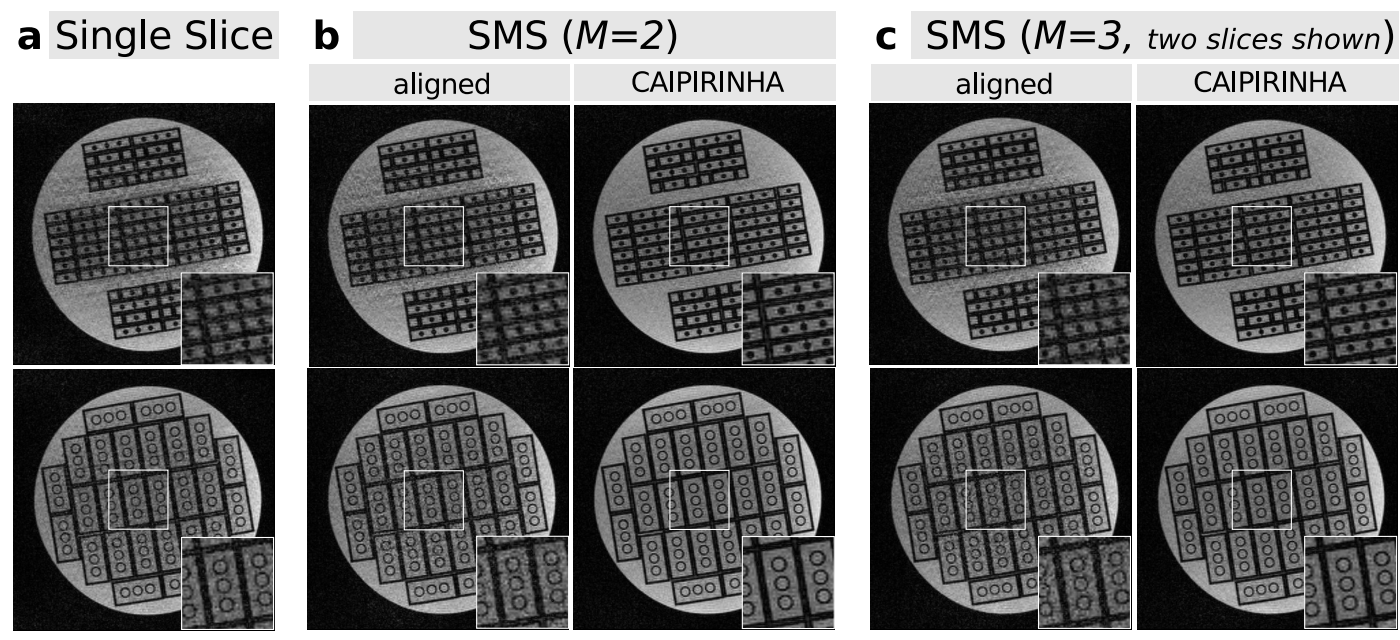

Figure 5.5.: Comparison of different acquisition and reconstruction strategies for Cartesian measurements on the brick phantom with reduction factor $R=4$ and $L_{\mathrm{ref}}=12$ reference lines. (a) Single-slice acquisition and NLINV reconstruction for each slice. (b) SMS acquisition and SMS-NLINV reconstruction for $M=2$ and aligned (left) and CAIPIRINHA pattern (right). Slice distance $d=60 \mathrm{~mm}$. (c) SMS acquisition and SMSNLINV reconstruction for $M=3$ and aligned (left) and CAIPIRINHA pattern (right). Only the outermost slices with slice distance $d=60 \mathrm{~mm}$ are depicted. A magnified ROI indicated by a white rectangle is shown as inset on the bottom right of every image.

\subsubsection{Radial Data}

Figure 5.6 depicts SMS-NLINV reconstructions of aligned, linear-turn- and goldenangle-turn-based radial SMS acquisitions with multi-band factor $M=3$ after it $=10$ Newton steps, as well as NLINV reconstructions of single-slice measurements for the same slices using it $=10$ Newton steps. The results for $M=2$ are provided in Figure 5.9. Similar to Figure 5.5 the slice images could be reconstructed without 
significant undersampling or superposition artifacts. As in the Cartesian case, the turn-based SMS acquisitions, where complementary k-space data are acquired in each partition, yield a much better image quality than aligned SMS and single-slice measurements. The linear-turn and the golden-angle-turn scheme yield similar results. As Figure 5.10 we provide difference images in image and k-space for $N_{\mathrm{sp}}=301$ and $N_{\mathrm{sp}}=29$ spokes per partition for the linear-turn-based $M=3$ measurement. Figure 5.11 shows the same experiments as Figure 5.6 and Figure 5.9 but with $N_{\mathrm{sp}}=69$ spokes.
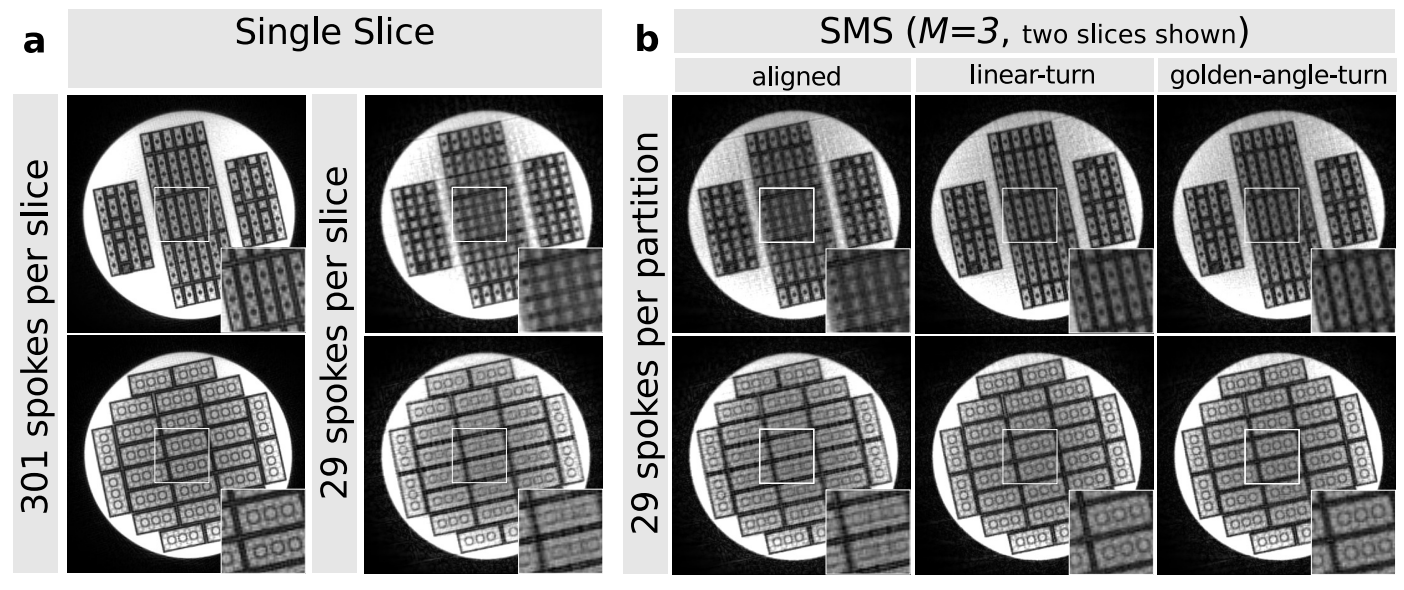

Figure 5.6.: Comparison of different acquisition and reconstruction strategies for radial measurements on the brick phantom with $N_{\mathrm{sp}}=29$ spokes per partition or slice and a fully sampled reference scan with $N_{\mathrm{sp}}=301$ spokes per slice. (a) Single-slice acquisition and NLINV reconstruction for each slice. (b) SMS acquisition and SMS-NLINV reconstruction for $M=3$ and aligned (left), linear-turn-based (center) and golden-angleturn-based sampling (right). Only the outermost slices with slice distance $d=60 \mathrm{~mm}$ are depicted. A magnified ROI indicated by a white rectangle is shown as inset on the bottom right of every image. The same experiment for $M=2$ is provided as Figure 5.9.

Figure 5.7 and Figure 5.8 show the results of the in vivo scans where we have chosen the number of Newton steps to obtain the best results using visual observation. Figure 5.7 shows $M=5$ slices of a 7 -fold undersampled acquisition of a human brain reconstructed with SMS-NLINV using it $=11$ Newton steps and ESPIRiT. Both methods show similar results as all slices are completely disentangled and all streaking artifacts could be eliminated. As Figure 5.12 shows, the residual for the SMS-NLINV reconstruction approaches a constant value when plotted against the number of Newton steps.

Figure 5.8 depicts $M=2$ slices of a human heart in end-diastole simultaneously acquired in $154 \mathrm{~ms}$ using $N_{\mathrm{sp}}=35$ spokes per partition and reconstructed with SMS-NLINV using it $=13$ Newton steps. Again, the two slices are completely disentangled and only slight blurring as well as minor streaking artifacts are present. 


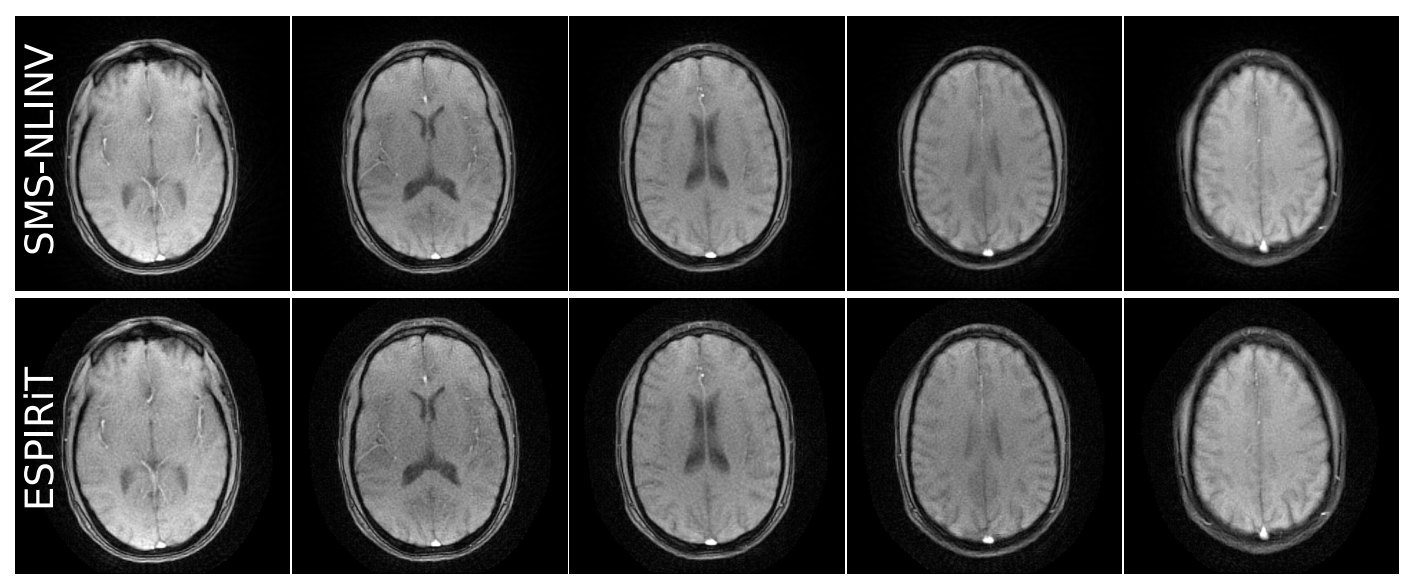

Figure 5.7.: SMS-NLINV and ESPIRiT reconstructions of a human brain using a radial SMS-FLASH acquisition with multi-band factor $M=5$ (slice distance $d=10 \mathrm{~mm}$, slice thickness $\Delta z=4 \mathrm{~mm}$, golden-angle-turn scheme, $N_{\mathrm{sp}}=39$ spokes per partition, $i t=11$ Newton steps).

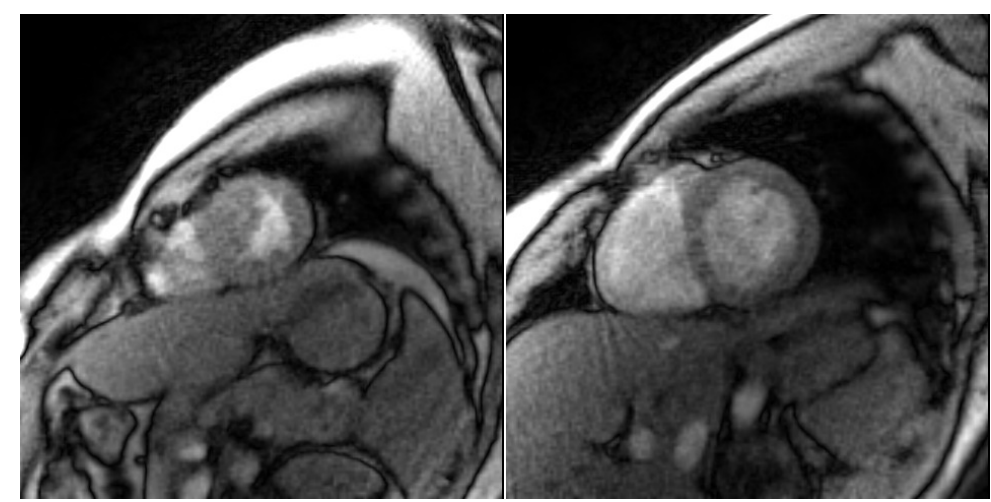

Figure 5.8.: SMS-NLINV reconstruction of a human heart in end-diastole using a realtime SMS-FLASH acquisition with multi-band factor $M=2$ (slice distance $d=40 \mathrm{~mm}$, slice thickness $\Delta z=6 \mathrm{~mm}, N_{\mathrm{sp}}=35$ spokes per partition, $i t=13$ Newton steps). 


\subsection{DISCUSSION}

In this work, SMS-NLINV has been combined with a Cartesian and a radial SMSFLASH sequence.

With sufficient reference lines, SMS-NLINV and ESPIRiT reconstruct undersampled SMS data with similar image quality as shown in this work for radial and Cartesian data. This finding is in agreement with previous results comparing ESPIRiT and regular NLINV [96]. For very small calibration regions SMS-NLINV still provides good results where ESPIRiT reconstructions already show severe artifacts. The reason for this is that SMS-NLINV does not rely on a fully sampled calibration region but jointly estimates the image content and the coil sensitivities. In contrast, direct calibration using ESPIRiT or other calibration methods requires a complicated four step procedure and only the region in k-space, which fulfills the Nyquist criterion simultaneously in all partitions can be utilized for calibration. SMS-NLINV not only makes all these additional separate processing steps unnecessary, it also works even for very small calibration regions by exploiting all available samples. This latter property makes SMS-NLINV ideally suited for non-Cartesian sampling, especially for accelerated dynamic imaging with changing coil sensitivities where only five to seven spokes per partition are acquired and the Nyquist-sampled region can become very small [62]. We compared Cartesian SMS acquisitions with CAIPIRINHA and aligned patterns as well as radial SMS acquisitions with aligned spoke scheme and linear-turn spoke schemes for different multi-band factors $M$ with single-slice $(M=1)$ measurements as control. The aligned schemes acquire the same k-space samples for each of the $M$ partitions, which resembles an averaging process and thus yields an SNR benefit relative to single-slice measurements. However, the partitions do not contain complementary k-space information and therefore no significant advantages in terms of better resolved details can be achieved. In this case a joint reconstruction does not possess any benefits compared to an inverse discrete Fourier transform (Equation (5.4)) on the Fourier-encoded k-spaces followed by single-slice reconstructions. The actual advantage of SMS-NLINV becomes apparent with the use of schemes where each partition contributes complementary k-space data, which is equivalent to supplementary object information. Consequently, in addition to the SNR benefit, details are better resolved. Whereas single-slice NLINV and aligned SMS-NLINV recover missing k-space samples using $2 \mathrm{D}$ sensitivity information, SMS-NLINV using complementary data also exploits sensitivity variations in the third dimension, which allows for higher acceleration factors [216].

In principle, the more slices $M$ we simultaneously acquire using an interleaved scheme, the better will be the resulting image quality of each slice due to the $\sqrt{M}$-like SNR benefit and the acquisition of additional complementary samples. However, for the acquisition of $M$ slices we have to perform $M$ partition measure- 
ments and therefore the measurement time increases with increasing $M$ until it approaches the time of a three-dimensional measurement. The optimal choice for $M$ depends on various experimental considerations such as overall motion robustness, magnetization preparation scheme, etc.

A future subject of study will be the use of SMS-NLINV for dynamic imaging at high temporal resolution, which was already successfully demonstrated for singleslice MRI using NLINV [41-43, 222]. The incorporation of additional minimization penalties such as temporal regularization and median filtering [41] known from NLINV and adapted spoke distribution schemes for dynamic SMS imaging [223] can directly be applied to SMS-NLINV and will further reduce streaking artifacts as well as blurring and improve the overall image quality. Preliminary results have been presented by Rosenzweig et al. [62].

In this work, we used a basic SMS-FLASH sequence. However, SMS-NLINV is a very general reconstruction approach and should be applicable to all sequences that can make use of an SMS acquisition, such as Diffusion Tensor Imaging, functional $M R I$ or $T_{1} / T_{2}$ quantification. In the future, we also plan to combine SMS-NLINV with a bSSFP sequence [158] and more advanced regularization techniques, which will improve image quality at high acceleration [224, 225].

\subsection{CONCLUSION}

The present work extends the NLINV algorithm to SMS MRI. As NLINV does not rely on the presence of Cartesian calibration data, it is an ideal choice for parallel imaging with non-Cartesian acquisitions. The combination with SMS offers the advantages of increased SNR and higher acceleration by exploiting three-dimensional sensitivity encoding. 


\subsection{APPENDIX}

\subsubsection{Glossary}

Table 5.1.: Glossary of notations

\begin{tabular}{clcl}
\hline$M$ & Multi-band factor & $N$ & Number of receive channels \\
$d$ & Slice distance & $\boldsymbol{x}_{q}$ & Magnetization and coil sensitivities \\
$\boldsymbol{y}$ & k-spaces of all coils & & for slice $q$ \\
$y^{j}$ & k-space of coil $j$ & $y_{q}$ & k-space of slice $q$ \\
$\boldsymbol{m}$ & Magnetizations seen by all coils & $m^{j}$ & Magnetization seen by coil $j$ \\
$m_{q}$ & Magnetization of slice $q$ & $\boldsymbol{c}$ & Coil sensitivities of all coils \\
$c_{q}$ & Coil sensitivity of slice $q$ & $\hat{c}$ & Normalized coil sensitivity \\
$z_{q}$ & Center coordinate of slice $q$ & $\Delta z$ & Slice thickness \\
$\sim$ & Encoded quantity & $H$ & Adjoint \\
$T$ & Transpose & $*$ & Complex conjugate \\
$i t$ & Number of Newton steps & & \\
\hline
\end{tabular}

\subsubsection{Derivative and Adjoint of the Forward Operator}

Given the forward operator $F(x)$ from Equation (5.6) the corresponding derivative reads

$$
\left.D F\right|_{X}\left(\begin{array}{c}
d \boldsymbol{x}_{1} \\
\vdots \\
d \boldsymbol{x}_{M}
\end{array}\right)=\boldsymbol{P} \Xi\left(\begin{array}{c}
\mathcal{F}\left(d m_{1} \boldsymbol{c}_{1}+m_{1} d \boldsymbol{c}_{1}\right) \\
\vdots \\
\mathcal{F}\left(d m_{M} \boldsymbol{c}_{M}+m_{M} d \boldsymbol{c}_{M}\right)
\end{array}\right)
$$

The adjoint of the derivative is given by

$$
\left.D F^{H}\right|_{X}\left(\begin{array}{c}
d \tilde{\boldsymbol{y}}_{1} \\
\vdots \\
d \tilde{\boldsymbol{y}}_{M}
\end{array}\right)=\left(\begin{array}{ccc}
\left(\begin{array}{c}
\boldsymbol{c}_{1}^{H} \\
m_{1}^{H}
\end{array}\right) & & 0 \\
& \ddots & \\
& & \\
0 & & \left(\begin{array}{c}
\boldsymbol{c}_{M}^{H} \\
m_{M}^{H}
\end{array}\right)
\end{array}\right) \mathcal{F}^{H} \Xi^{H} \boldsymbol{P}^{H}\left(\begin{array}{c}
d \tilde{\boldsymbol{y}}_{1} \\
\vdots \\
d \tilde{\boldsymbol{y}}_{M}
\end{array}\right),
$$

with

$$
\left(\begin{array}{c}
c_{q}^{H} \\
m_{q}^{H}
\end{array}\right):=\left(\begin{array}{c}
c_{q}^{1^{*}}, \ldots, c_{q}^{N^{*}} \\
m_{q}^{*}
\end{array}\right)
$$

The asterisk $*$ denotes pointwise complex conjugation. 


\subsubsection{Weighting Matrix}

The weighting matrix used in SMS-NLINV to implement the smoothness penalty for the coil sensitivities is given by:

$$
W^{-1}:=\left(\begin{array}{ccc}
W^{-1} & & 0 \\
& \ddots & \\
0 & & W^{-1}
\end{array}\right),
$$

Here, $W^{-1}$ is the same weighting matrix as used in conventional NLINV:

$$
W^{-1}:=\left(\begin{array}{cccc}
I & & & 0 \\
& a\left(1+b|| \vec{k} \|^{2}\right)^{l / 2} \mathcal{F} & & \\
& & \ddots & \\
0 & & & a\left(1+b\|\vec{k}\|^{2}\right)^{l / 2} \mathcal{F}
\end{array}\right) .
$$

\subsection{SUPPLEMENTARY MATERIAL}
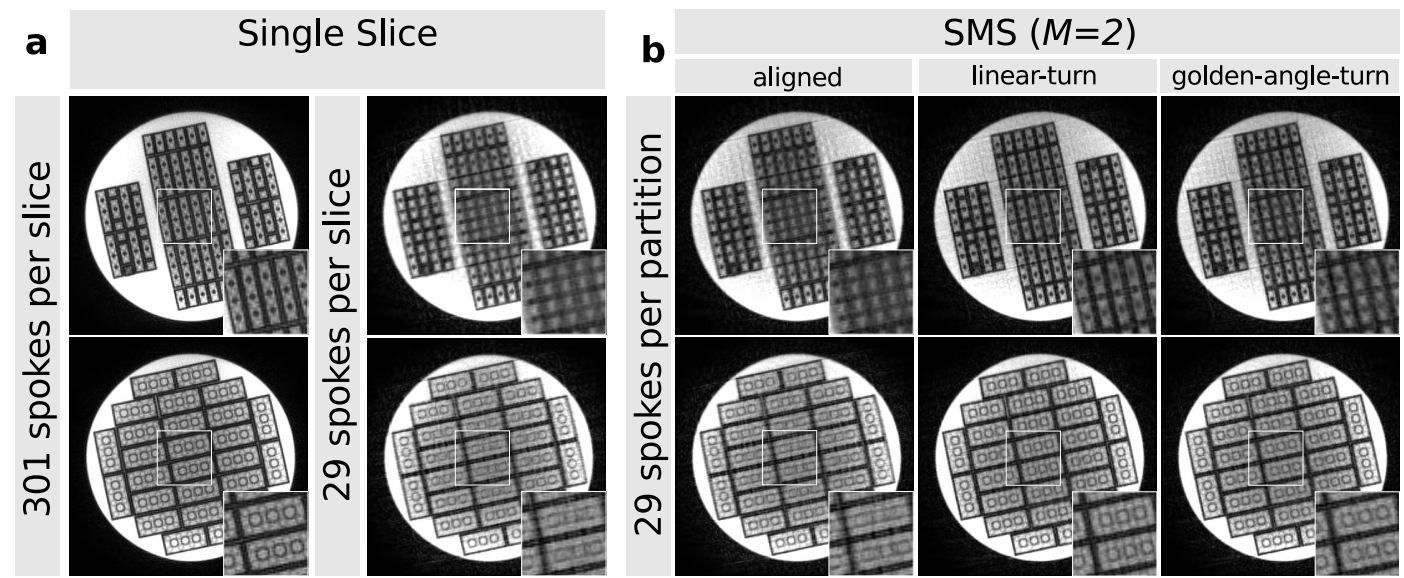

Figure 5.9.: Comparison of different acquisition and reconstruction strategies for radial measurements on the brick phantom with $N_{\mathrm{sp}}=29$ spokes per partition or slice and a fully sampled reference scan with $N_{\mathrm{sp}}=301$ spokes per slice. (a) Single-slice acquisition and NLINV reconstruction for each slice. (b) SMS acquisition and SMS-NLINV reconstruction for $M=2$ and aligned (left), linear-turn-based (center) and golden-angleturn-based sampling (right). Slice distance $d=60 \mathrm{~mm}$. A magnified ROI indicated by a white rectangle is shown as inset on the bottom right of every image. 


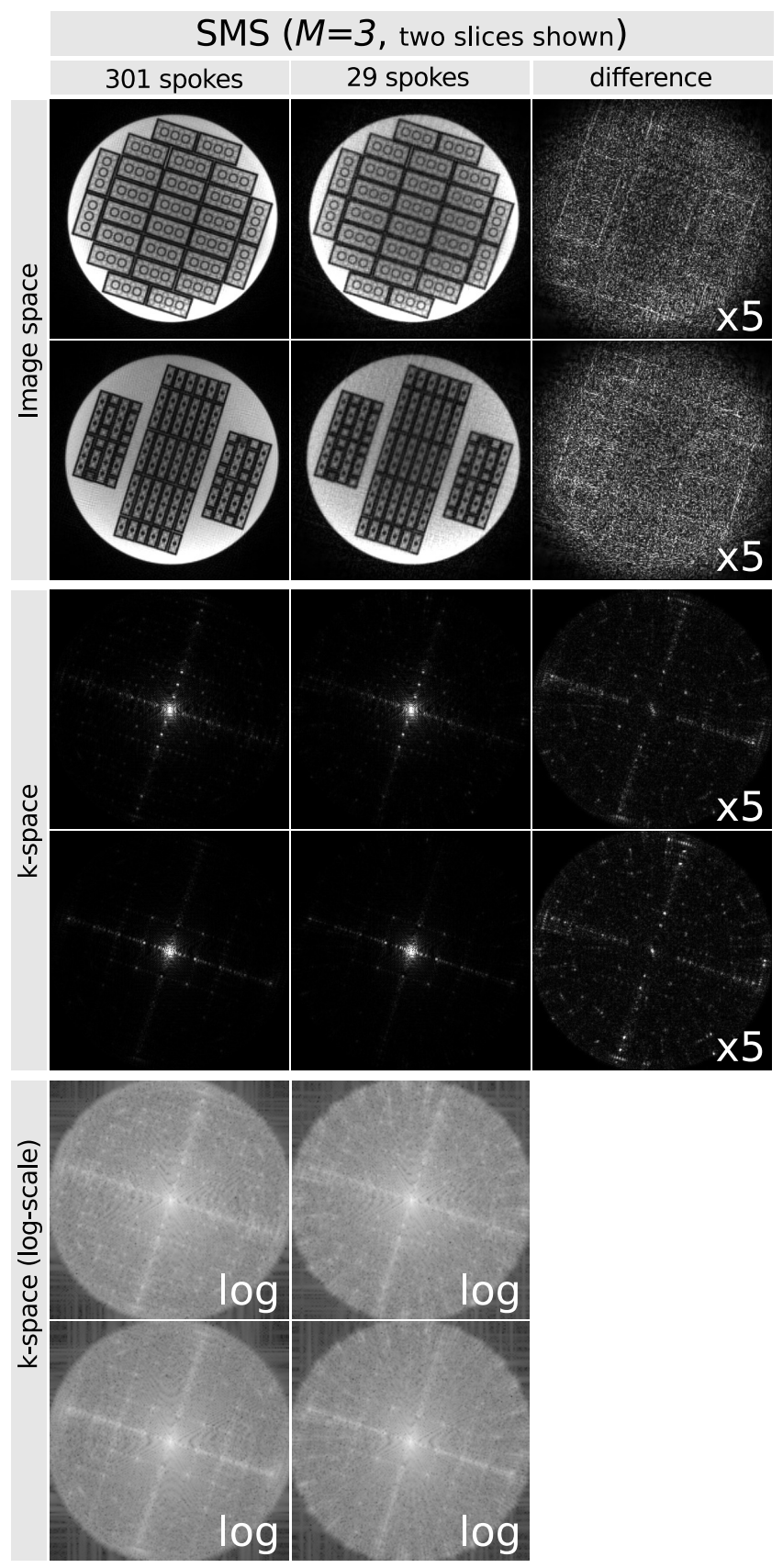

Figure 5.10.: Difference images in image and k-space for SMS $(M=3$, slice distance $d=30 \mathrm{~mm}$, linear-turn-based spoke distribution) acquisitions with $N_{\mathrm{sp}}=301$ (fully sampled reference) and $N_{\mathrm{sp}}=29$ spokes per partition. For better visibility, the intensity of the difference images was increased by a factor of 5 and the k-spaces were additionally depicted using the log-scale. 
a
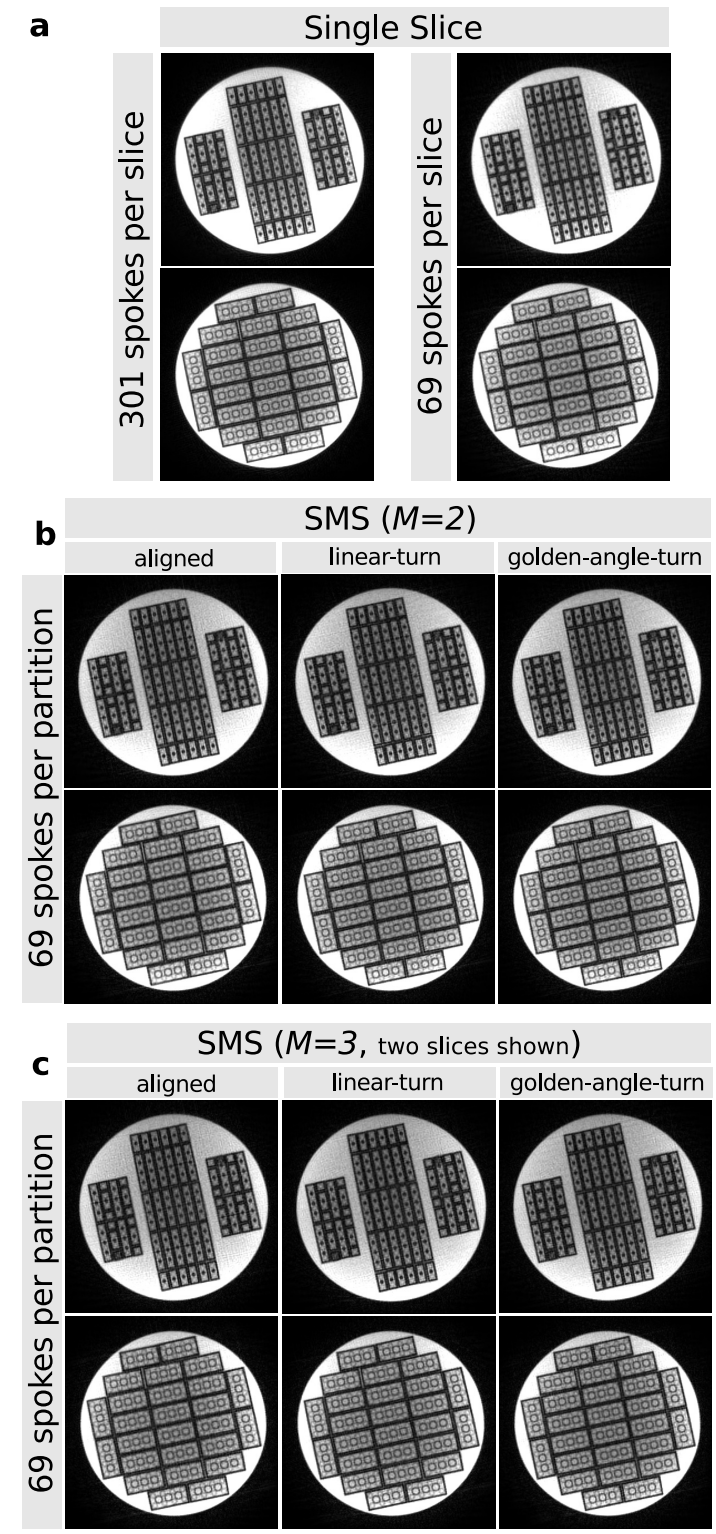

Figure 5.11.: Comparison of different acquisition and reconstruction strategies for radial measurements on the brick phantom with $N_{\mathrm{sp}}=69$ spokes per partition or slice and a fully sampled reference scan with $N_{\mathrm{sp}}=301$ spokes per slice. (a) Single-slice acquisition and NLINV reconstruction for each slice. (b) SMS acquisition and SMSNLINV reconstruction for $M=2$ and aligned (left), linear-turn-based (center) and golden-angle-turn-based sampling (right). Slice distance $d=60 \mathrm{~mm}$. (c) SMS acquisition and SMS-NLINV reconstruction for $M=3$ and aligned (left), linear-turn-based (center) and golden-angle-turn-based sampling (right). Only the outermost slices with slice distance $d=60 \mathrm{~mm}$ are depicted. 


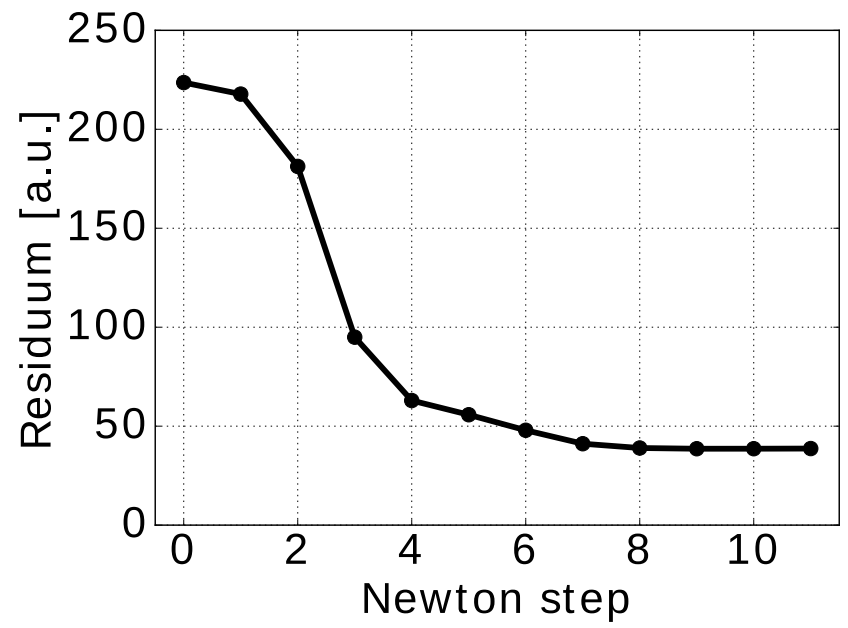

Figure 5.12.: Residuum of the SMS-NLINV reconstruction in Figure 5.7 against the number of Newton steps. 



\section{REAL-TIME IMAGING USING SMS-NLINV}

In the previous chapter, we have demonstrated the capability of SMS-NLINV to provide accurate reconstructions from highly undersampled SMS data. The imaging of dynamic processes allows for even higher undersampling factors by exploiting temporal correlations. In this chapter, we will therefore incorporate temporal regularization into the SMS-NLINV algorithm to employ radial SMS-NLINV for real-time imaging of $M=3$ and $M=5$ simultaneously acquired slices. We will compare the results to conventional interleaved multi-slice measurements.

Parts of this work have been published as an abstract at the ISMRM 25th and 26th Annual Meeting and Exhibition in 2017 and 2018 [61, 62].

\subsection{THEORY}

The effective use of temporal regularization requires adapted radial acquisition schemes, as Figure 2.15 demonstrates. Therefore, the acquisition scheme for realtime single-slice imaging presented in Section 2.5.2 must be revised for real-time SMS MRI and conventional time-resolved interleaved multi-slice MRI.

For the latter, the pattern with inter-frame rotations illustrated in Figure 2.14a (bottom row) is commonly utilized with a small modification. Instead of performing conventional single-slice acquisitions one slice after the other, a spoke from a different slice is recorded in each TR. This strategy, which is illustrated in Figure 6.1a, enforces temporal consistency at the expense of reduced temporal resolution for each slice, as only every $M$-th TR a spoke of the same slice is acquired. To obtain a uniform k-space coverage for a single frame and slice, the angle increment is set to $\alpha_{\mathrm{sp}}=2 \pi / N_{\mathrm{sp}}$, with $N_{\mathrm{sp}}$ the number of spokes per frame. The resulting spoke distribution is rotated by $\alpha_{\text {fra }}=\alpha_{\mathrm{sp}} / N_{\text {pat }}$ to obtain $N_{\text {pat }}=5$ different frame patterns, which are repeated for succeeding frames.

While for interleaved multi-slice experiments only one slice per TR is excited, for SMS MRI in each TR all slices are simultaneously excited. Accordingly, each spoke contains superposed information from all slices, for which spokes do not belong to specific slices but to partitions, which represent $z$-encodings in k-space. Consecutive spokes are acquired from different partitions, thus the projection angle is increased by $\alpha_{\mathrm{trn}}=\pi /\left(N_{\mathrm{sp}} \cdot M\right)$ until all partitions have been visited, which is motivated by Chapter 5 and Equation (5.13). As before, the angle increment within a frame and partition is set to $\alpha_{\mathrm{sp}}=2 \pi / N_{\mathrm{sp}}$. To generate $N_{\mathrm{pat}}=5$ different 
frame patterns, the spoke distribution for all partitions of a frame is rotated in the subsequent frame by $\alpha_{\mathrm{fra}}^{\mathrm{SMS}}=2 \pi /\left(N_{\mathrm{sp}} \cdot M \cdot N_{\text {pat }}\right)$. This strategy avoids redundancy and guarantees uniform k-space coverage within $N_{\text {pat }}=5$ consecutive frames. The scheme is repeatedly applied for succeeding frames. Figure 6.1b and Figure 6.1c illustrate this approach.

For consistent disentangling of the superposed slices, $M$ consecutive spokes are assigned to the same time-step, for which the temporal resolution of SMS MRI is equal to the corresponding interleaved multi-slice experiment. Nevertheless, the benefits of SMS MRI, such as improved SNR and the ability to exploit spatial correlations between slices as described in Chapter 5 give reason to expect quality improvements.

As proposed in [42] and described in Section 2.5.2, we incorporate temporal regularization in the SMS-NLINV algorithm by modification of the objective function Equation (5.12) to take advantage of the complementary k-space information introduced by the frame-wise rotated spoke patterns. Let $X^{\prime(t)}$ be the weighted vector of image content and coil sensitivities for time-step $t$. Then, temporal regularization is incorporated by penalizing the difference of iteration $n$ of the current frame $X_{n}^{\prime(t)}+d X^{\prime(t)}$ and the result of the previous frame $X^{\prime(t-1)}$, damped by the factor $\xi=0.9$.

$$
\begin{aligned}
\Phi\left(d X^{\prime(t)}\right)=\underset{d X^{\prime(t)}}{\operatorname{argmin}}( & \left\|\left.D G\right|_{X_{n}^{\prime(t)}} d X^{\prime(t)}-\left(\tilde{Y}^{(t)}-G X_{n}^{\prime(t)}\right)\right\|_{L^{2}}^{2} \\
& \left.+\beta_{n}\left\|X_{n}^{\prime(t)}+d X^{\prime(t)}-\xi X^{\prime(t-1)}\right\|_{L^{2}}^{2}\right),
\end{aligned}
$$

Intuitively, this enforces similarity between the current and the previous frame. To reduce the number of required IRGNM steps and thus to speed-up the image reconstruction, the previous frame can furthermore be used as initialization. 
(a) Interleaved Multi-Slice

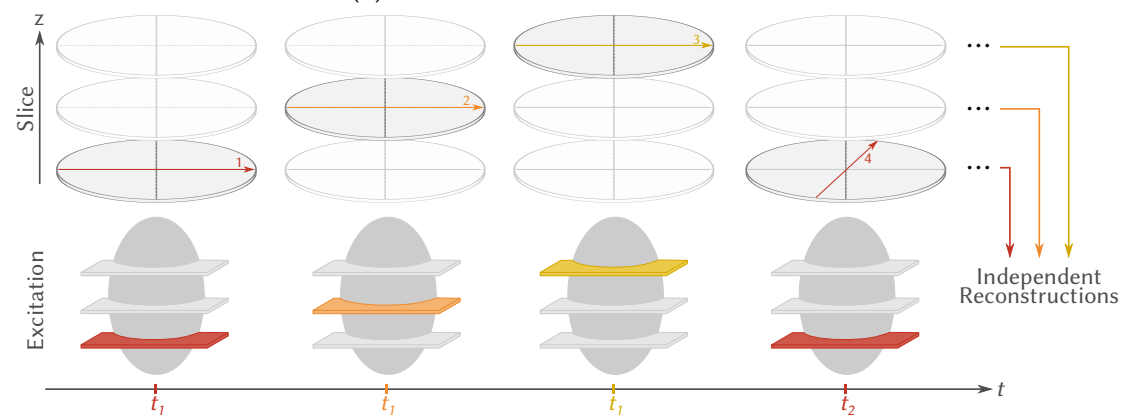

(b) Simultaneous Multi-Slice (SMS)
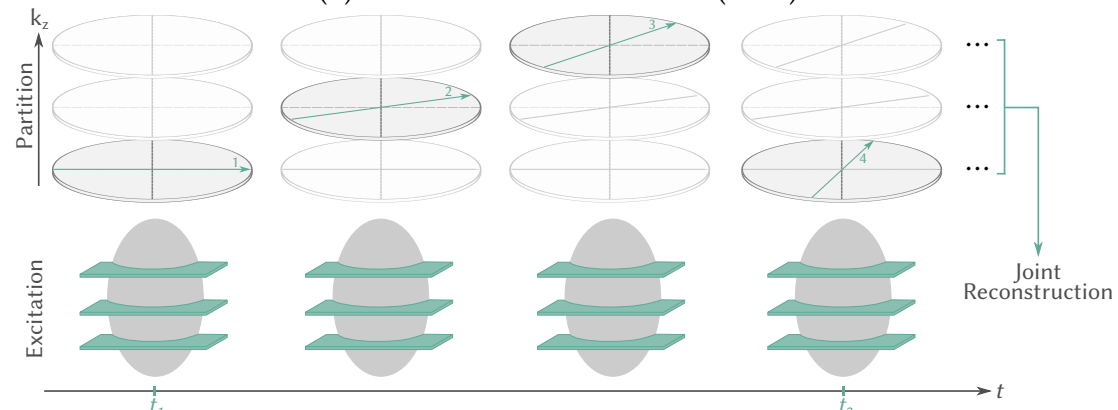

(c) SMS Rotation Scheme
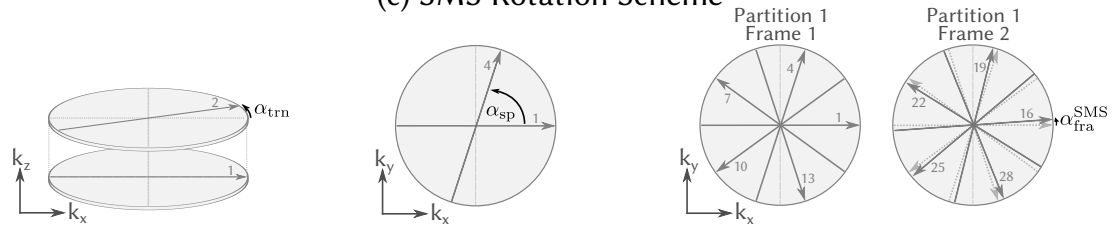

Figure 6.1.: Illustration of a time-resolved interleaved radial multi-slice and SMS acquisition scheme for three slices. The top row of (a) and (b) depicts the k-space pattern and in the bottom row, colored planes highlight the excited slices per TR. (a) For interleaved multi-slice MRI, one slice at a time is excited and a single spoke is acquired before proceeding to the next slice, where a spoke with the same projection angle is recorded. After a completed iteration through all slices, the projection angle is increased by $\alpha_{\mathrm{sp}}$ and the procedure is repeated. When all spokes per frame and slice have been acquired, the spoke pattern is rotated as in Figure 2.14 to increase the $\mathrm{k}$-space coverage (not shown here). The temporal increment between two acquired spokes within a slice is here given by $\Delta t=3 \mathrm{TR}$. The numbers next to the spokes indicate the acquisition order. (b) For SMS MRI, all slices are excited in each TR using a specific encoding. Thus, the corresponding spoke does not belong to a particular slice but to a partition, i.e. a $k_{z}$-position in k-space, and contains information about all slices. This increases the SNR compared to (a). Three consecutive spokes are combined to one time-step, which yields the same temporal increment $\Delta t=3 \mathrm{TR}$ as in (a). Subfigure (c) depicts the angle $\alpha_{\mathrm{sp}}$ between spokes within one partition. While iterating through the partitions the projection angle is increased by $\alpha_{\mathrm{trn}}$, which extends the k-space coverage compared to interleaved multi-slice. When all spokes per frame and partition have been acquired, the entire spoke pattern for all partitions is rotated by $\alpha_{\text {fra }}^{\text {SMS }}$ for temporal complementarity. 


\subsection{METHODS}

In this proof of principle study for real-time cardiac SMS imaging, we conducted SMS-FLASH measurements of 10 s duration with $M=3$ and $M=5$ slices. For comparison, we performed conventional interleaved multi-slice measurements of the same slices and with similar temporal resolution. The interleaved multi-slice measurements were reconstructed individually using NLINV with temporal regularization. A joint reconstruction of all slices was performed for the SMS acquisition using SMS-NLINV with temporal regularization.

For all measurements the following parameters were used: Slice thickness $5 \mathrm{~mm}$, slice gap $10 \mathrm{~mm}$, TE/TR $1.35 / 2.10 \mathrm{~ms}$, flip angle $8^{\circ}$, base resolution 128 and FOV $256 \times 256 \mathrm{~mm}^{2}$. We utilized the spoke distribution schemes presented in Section 6.1 with $N_{\mathrm{sp}}=5$ spokes per partition/slice for the $M=3$ experiment and $N_{\mathrm{sp}}=3$ spokes per partition/slice for the $M=5$ experiment, which yields a temporal resolution of $31.5 \mathrm{~ms}$ per frame and slice for all experiments. Gradient delay correction was performed using RING [58]. For the interleaved multi-slice experiments, gradient delays were estimated individually for each slice. For the SMS experiments the center partition, which contains most energy, was used to determine a gradient delay estimate, that was then used to correct all partitions.

\subsection{RESULTS}

Figure 6.2a depicts end-systolic frames for all three slices of the SMS measurement, as well as the temporal evolution of a vertical line extracted from the center slice. Figure $6.2 \mathrm{~b}$ shows the same plot for the interleaved multi-slice experiment. In both spatio-temporal plots, eight full heartbeats can be recognized. Furthermore, approximately two respiration cycles can be appreciated. The overall image quality as well as the time resolved performance is comparable for both experiments. Nevertheless, the blood to myocardium contrast of the SMS acquisition outperforms the conventional multi-slice results, which is even more apparent in the experiments using five slices shown in Figure 6.2c and Figure 6.2d. Still, the quality of both five-slice experiments is significantly decreased and appear more blurry and unsharp compared to the results for three slices. 
(a) SMS (3 slices)

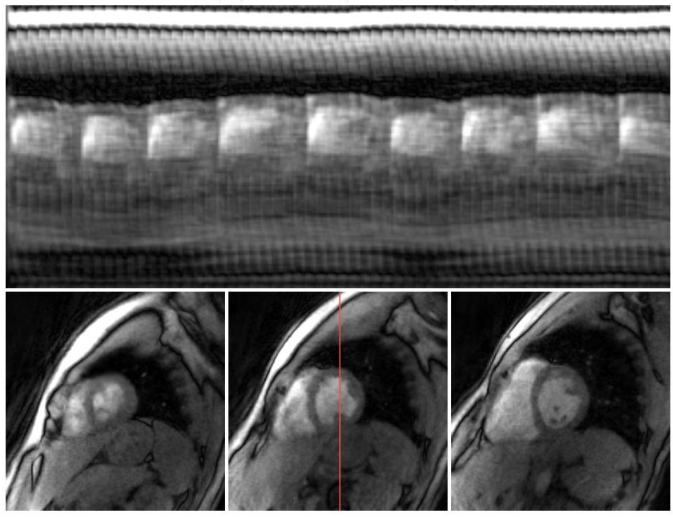

(b) Interleaved Multi-Slice (3 slices)
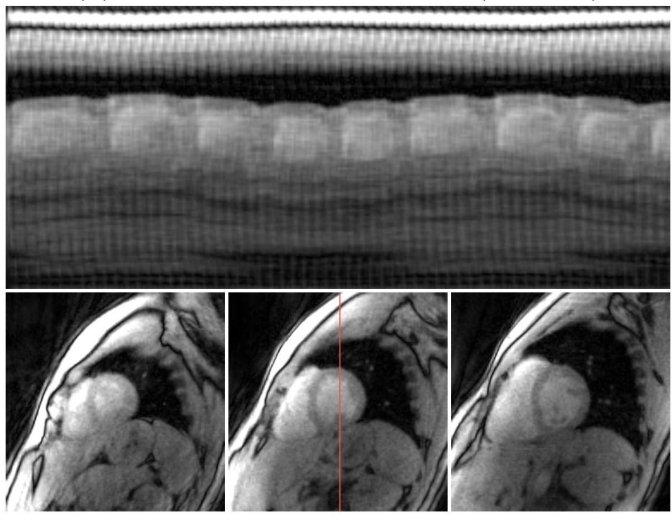

(c) SMS (5 slices)

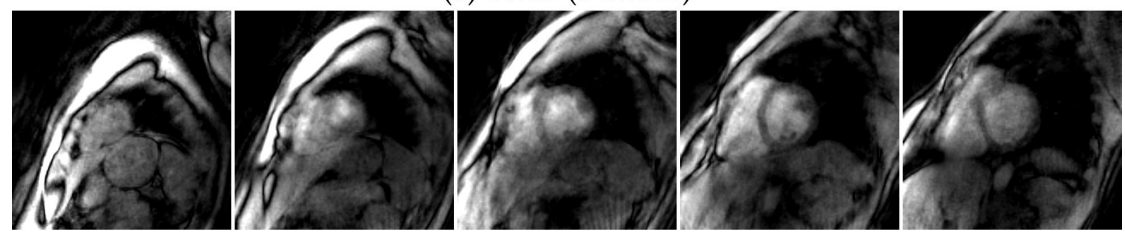

(d) Interleaved Multi-Slice (5 slices)

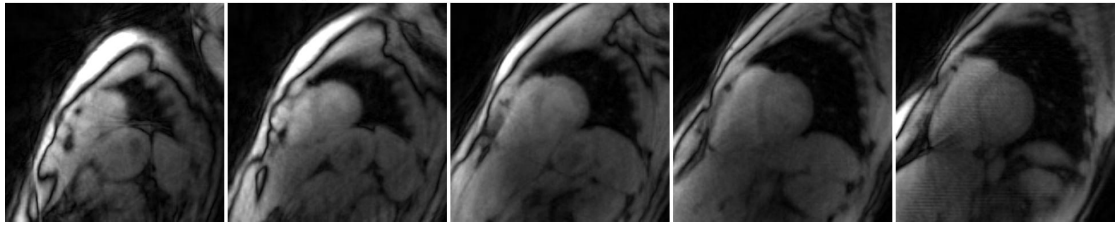

Figure 6.2.: Results of the real-time SMS $(\mathrm{a}, \mathrm{c})$ and real-time interleaved multi-slice experiments (b,d) with three and five slices. (a) and (b) depict end-diastolic frames for all three slices. Moreover, the temporal evolution of a vertical line (red highlight) extracted from the center slice is shown. In (c) and (d) end-diastolic frames of all five slices are displayed. 


\subsection{DISCUSSION}

The presented results suggest that both three-slice experiments with five spokes per frame and slice/partition achieve a reasonable image quality with a clinically relevant temporal resolution. In contrast, the excessive undersampling in the fiveslice experiment with three spokes per frame and slice/partition distinctly deteriorates the results.

Due to the repeated excitation of all slices in each TR, the SNR is expected to be increased by a factor of $\sqrt{M}$ in the SMS measurement [63]. However, the use of spatial and temporal regularization has a smoothing effect and hampers a proper SNR analysis. Moreover, the SNR is affected by the choice of the flip angle. A flip angle of $8^{\circ}$ was proposed for cardiac radial single-slice FLASH [42] and provides a good blood to myocardium contrast in end-diastole for SMS experiments. However, for interleaved multi-slice MRI, the effective TR for each slice is increased by a factor of $M$, which alters the steady-state condition. Therefore, a higher flip angle might be more appropriate and could increase the blood to myocardium contrast in end-diastole.

This being said, the use of the FLASH sequence for interleaved multi-slice or SMS imaging of the heart is not recommended for a routine clinical use. The blood to myocardium contrast in the FLASH sequence relies on the in-flow of fresh, unsaturated blood. The repeated excitation of multiple slices inside the heart impairs this condition. Beyond that, the bSSFP sequence provides a better blood to myocardium contrast and higher SNR and is therefore often preferred for cardiac MRI [226]. However, conventional interleaved multi-slice MRI is incompatible with a bSSFP sequence, since the condition TE $=\mathrm{TR} / 2$ is infeasible due to the prolonged effective TR. Therefore conventional multi-slice bSSFP imaging is limited to a sequential acquisition of one slice after the other, which inhibits inter-slice time-consistency. On the contrary, SMS imaging can be combined with the bSSFP approach [158, 159], which also solves the problem of contrast loss in end-systolic phases.

\subsection{CONCLUSION}

In this study, we have successfully applied SMS-NLINV for the reconstruction of real-time SMS measurements on a human heart. A detailed study to investigate the effects of different parameter combinations such as flip angle or number of spokes per frame and the implementation of the SMS bSSFP sequence was beyond the scope of this thesis and remains future work. 


\section{CARDIAC AND RESPIRATORY SELF-GATING IN RADIAL MRI USING AN ADAPTED SINGULAR SPECTRUM ANALYSIS (SSA-FARY)}

The following is a reprint of the original article

S. Rosenzweig, N. Scholand, H. C. M. Holme, and M. Uecker. "Cardiac and Respiratory Self-Gating in Radial MRI using an Adapted Singular Spectrum Analysis (SSA-FARY)". IEEE Trans. Med. Imag. (2020). doi: 10.1109/TMI.2020.2985994.

(C) 2020 IEEE. Reprinted, with permission from [227].

$S R$ designed the SSA-FARY method under supervision from $M U$ and implemented it with input from NS and $C H$. SR implemented the SOS sequence with the help of $C H$. SR designed all experiments with guidance from $M U$. SR conducted all experiments and NS assisted with the execution. $S R$ realized the analysis of the results with input from NS and $M U$. SR wrote most of the manuscript and all authors contributed to its preparation.

The layout, the reference numbering, the orthography and the use of acronyms have been adapted to preserve the natural text flow of this thesis. No substantive changes to the content have been realized.

In reference to IEEE copyrighted material which is used with permission in this thesis, the IEEE does not endorse any of Georg-August-University's products or services. Internal or personal use of this material is permitted. If interested in reprinting/republishing IEEE copyrighted material for advertising or promotional purposes or for creating new collective works for resale or redistribution, please go to http://www.ieee.org/ publications_standards/publications/rights/rights_link.html to learn how to obtain a License from RightsLink. If applicable, University Microfilms and/or ProQuest Library, or the Archives of Canada may supply single copies of the dissertation. 


\section{ABSTRACT}

Cardiac Magnetic Resonance Imaging (MRI) is time-consuming and errorprone. To ease the patient's burden and to increase the efficiency and robustness of cardiac exams, interest in methods based on continuous steady-state acquisition and self-gating has been growing in recent years. Self-gating methods extract the cardiac and respiratory signals from the measurement data and then retrospectively sort the data into cardiac and respiratory phases. Repeated breathholds and synchronization with the heartbeat using some external device as required in conventional MRI are then not necessary. In this work, we introduce a novel self-gating method for radially acquired data based on a dimensionality reduction technique for time-series analysis (SSA-FARY). Building on Singular Spectrum Analysis, a zero-padded, timedelayed embedding of the auto-calibration data is analyzed using Principle Component Analysis. We demonstrate the basic functionality of SSA-FARY using numerical simulations and apply it to in vivo cardiac radial singleslice bSSFP and Simultaneous Multi-Slice radio-frequency-spoiled gradientecho measurements, as well as to Stack-of-Stars bSSFP measurements. SSAFARY reliably detects the cardiac and respiratory motion and separates it from noise. We utilize the generated signals for high-dimensional image reconstruction using parallel imaging and compressed sensing with in-plane wavelet and (spatio-) temporal total-variation regularization. 


\subsection{INTRODUCTION}

Magnetic Resonance Imaging (MRI) is an intrinsically slow imaging technique, which makes imaging of moving organs particularly challenging. Still, from the early years of MRI, researchers recognized the great chances and implications of monitoring the beating heart without the use of ionizing radiation and with the superior tissue contrast of MRI. Here, the respiratory and cardiac motion pose high demands on the acquisition and reconstruction.

One solution is real-time imaging, which resolves the true dynamics of the heart but is limited in terms of temporal and spatial resolution and restricted to twodimensional imaging [41, 42, 199, 201]. In clinical practice, pro- or retrospective gating is typically used, which exploits the quasi-periodicity of the respiratory and cardiac motion to compose a single synthetic heartbeat from data acquired during several actual beats. To synchronize data-acquisition with breathing motion, external devices like a respiratory belt or adapted sequences with navigator readouts are commonly used [144, 228]. However, these devices have to be placed and adjusted individually for each patient. Furthermore, the resulting respiratory signal is not always directly correlated to the motion of the heart [229]. Sequences with additional interleaved navigator acquisitions prolong the measurement substantially and complicate the use of steady-state sequences. Otherwise, breath-hold commands can be used to avoid the need for respiratory gating completely, which, however, can be exhausting, time-consuming and not expedient for sick or non-compliant patients and children. For cardiac gating, the standard in clinical practice is the use of an electrocardiogram (ECG) [230], but the ECG signals can experience signal distortion when MRI sequences with fast gradient switching are utilized [231].

To avoid these drawbacks and to gain more flexibility, techniques have been developed to extract cardiac motion from the data itself, which is known as retrospective self-gating [52]. Similar approaches can also be used to extract respiratory motion [51]. A large number of different strategies for cardiac, respiratory or combined self-gating with Cartesian or non-Cartesian acquisition were proposed in the past, e.g. [52-55, 57, 232]. Still, the fundamental idea in most approaches is similar: Either a $1 \mathrm{D}$ signal is extracted from certain receive channels using a bandpass filter and specific properties of the acquired auto-calibration (AC) data, or a (sliding window) low-spatial high-temporal resolution reconstruction of a specific Region of Interest (ROI) is analyzed. A more sophisticated yet simple idea was proposed by Pang et al. [56]: The general concept of dimensionality reduction [233] is applied to the AC data by using a Principle Component Analysis (PCA) to extract the required motion signals. However, the resulting signals are often spoiled by noise or trajectory-dependent oscillations, which makes additional filtering necessary $[61,143,234]$. Moreover, cardiac and respiratory motion are not always clearly separated [235], which complicates data binning into the respective breathing and 
heart phases and requires the use of further post-processing steps such as coil clustering [236].

To overcome these limitations, we propose the use of an adapted Singular Spectrum Analysis (SSA), which can be thought of as a temporally localized PCA or equivalently as a PCA applied to time-delay embedded coordinates. SSA is an application of the general Karhunen-Loève theorem [237] and a powerful tool for the analysis of dynamical systems, incorporating elements of classical time-series analysis, multivariate statistics, multivariate geometry and signal processing [238]. Broomhead and King derived SSA from Takens' theorem for the analysis of chaotic dynamical systems, and applied it to the problems of dynamical systems theory $[179,186]$. Further development was promoted by Vautard et al. [239, 240]. Since the birth of SSA in $1986[186,241]$ it has found wide-spread application in various fields [242-247]. SSA can be used for noise reduction, detrending and the identification of oscillatory components [240], hence it is ideally suited for the extraction of vital motion signals such as respiratory and cardiac motion in self-gated MRI.

Nevertheless, conventional univariate SSA can only be applied to single-channel time series, whereas in parallel MRI multiple receive channels (phased array coils) are available. Channels located closer to the heart tend to capture cardiac motion, while coils placed near the diaphragm rather monitor respiratory motion. Manual coil selection [248] can enable the use of univariate SSA for vital motion extraction, but correlated information from other coils is then lost. Moreover, for routine clinical use a fully automated technique is preferred.

Fortunately, univariate SSA has a natural extension for the analysis of a multichannel time series [241]. However, this multivariate SSA is not a dimensionality reduction technique, but recovers the specific oscillations for each channel rather than to extract a single signal that describes the temporal evolution of the principle motion components.

Here, we adapt the Singular Spectrum Analysis For Advanced Reduction of dimensionalitY, which we dub SSA-FARY $[64,65]$. In its original form (multivariate) SSA consists of four steps [238]: I) Hankelization, II) Decomposition, III) Grouping, IV) Backprojection. In SSA-FARY, we remove steps III) and IV) and instead perform a zero-padding operation at the start. We will demonstrate the basic functionality of SSA-FARY in numerical simulations and show reconstructions of in vivo cardiac measurements acquired with single-slice bSSFP and Simultaneous Multi-Slice (SMS) radio-frequency (RF)-spoiled gradient-echo (FLASH) sequences, as well as with a Stack-of-Stars (SOS) bSSFP sequence.

\subsection{THEORY}

In radial single-slice, SMS or SOS imaging the central k-space point or the central line along the slice-dimension $k_{z}\left(k_{x}=0, k_{y}=0\right)$, respectively, have proved 
to be ideally suited for self-gating $[52,143]$. We extract this AC data from the measurement data and stack all coils and partitions into a single dimension. This yields a multi-channel time-series $X_{c}^{t}$ of size $\left[\left(N_{p} \times N_{c}\right) \times N_{t}\right]$, with $1 \leq t \leq N_{t}$ and $1 \leq c \leq\left(N_{p} \cdot N_{c}\right)$, which contains information about the respiratory and cardiac motion. $N_{t}$ is the total number of central k-space points or lines used for autocalibration, $N_{p}$ is the number of partitions and $N_{c}$ is the number of receive coils. Each channel $c$ is normalized to have zero mean.

\subsubsection{Correction of the AC Data}

System imperfections such as gradient delays and off-resonances usually cause a corruption of the AC data $\boldsymbol{X}$, which manifests an oscillation of a trajectorydependent frequency in radial imaging [234]. This signal fluctuation is often misinterpreted by dimensionality reduction methods as a major signal contribution. This contribution can mostly be removed by a simple orthogonal projection based on its known frequency. Here, we extend this method to also include higher-order harmonics which yields a method that almost completely removes the unwanted signal. For simplicity, we assume a golden angle acquisition scheme. Let $\varphi_{\mathrm{o}}$ be the incremental projection angle, then

$$
\varphi^{t}=t \cdot \varphi_{\mathrm{o}}
$$

is the projection angle used for the acquisition at time step $t$. We define the vector

$$
n^{t}:=\left(\begin{array}{c}
e^{i \varphi^{t}} \\
e^{-i \varphi^{t}} \\
e^{i \cdot 2 \varphi^{t}} \\
e^{-i \cdot 2 \varphi^{t}} \\
\vdots \\
e^{i \cdot N_{H} \varphi^{t}} \\
e^{-i \cdot N_{H} \varphi^{t}}
\end{array}\right)
$$

containing the oscillations up to the $N_{H}$-th harmonic as a basis for the perturbing oscillation and constrain $\boldsymbol{X}$ to be orthogonal to $\boldsymbol{n}$,

$$
\boldsymbol{X}_{\text {cor }}=\boldsymbol{X}_{\text {raw }}-\boldsymbol{n}\left(\boldsymbol{n}^{\dagger} \boldsymbol{X}_{\text {raw }}\right),
$$

with $^{\dagger}$ denoting the pseudo-inverse. This procedure cleans the corrupted signal $\boldsymbol{X}_{\text {raw }}$ and yields a corrected time series $\boldsymbol{X}_{\text {cor }}$. We use this AC correction method in all presented in vivo experiments. 


\subsubsection{Dimensionality Reduction Methods}

PRINCIPLE COMPONENT ANALYSIS PCA can be understood as the rotation of the original coordinate system to a new one with orthogonal axes that coincide with the directions of maximum variable variance [249]. The PCA of a time series $\boldsymbol{X}$ can be performed using the Singular Value Decomposition (SVD).

$$
\boldsymbol{X}^{T}=\boldsymbol{U} \boldsymbol{S} \boldsymbol{V}^{H}
$$

Here, the diagonal matrix $S$ contains the real eigenvalues $\lambda_{1} \geq \cdots \geq \lambda_{N_{t}} \geq 0$ in decreasing order of magnitude. PCA provides the expansion of $\boldsymbol{X}^{T}$ onto the orthonormal $\left[N_{t} \times N_{t}\right]$ basis $\boldsymbol{U}$,

$$
\left(X^{T}\right)_{t}=\sum_{k=1}^{N_{t}} U_{t}^{k}\left(S V^{H}\right)_{k},
$$

where the principle components $\left(S V^{H}\right)_{k}$ are given by

$$
\left(S V^{H}\right)_{k}:=\sum_{l=1}^{N_{c} \cdot N_{p}} S_{k}^{l}\left(V^{H}\right)_{l}=\lambda_{k}\left(V^{H}\right)_{k} .
$$

Since the cardiac and respiratory motion signals contribute as main sources of variation to the time series $\boldsymbol{X}$, their temporal behavior should be captured by one of the first basis vectors $U^{k}$, respectively [56].

SINGULAR SPECTRUM ANALYSIS FOR ADVANCED DIMENSIONALITY REDUCTION (SSA-FARY) A schematic of the SSA-FARY procedure is depicted in Figure 7.1a. In contrast to conventional (multivariate) SSA, we first zero-pad $(\mathcal{Z})$ the second dimension of the AC data $\boldsymbol{X}$ to obtain matrix $\tilde{\boldsymbol{X}}$ of size $\left[\left(N_{p} \times N_{c}\right) \times\left(N_{t}+\right.\right.$ $W-1)]$

$$
\tilde{\boldsymbol{X}}=\boldsymbol{Z} \boldsymbol{X} .
$$

Next, we construct a Block-Hankel calibration matrix

$$
\boldsymbol{A}=\mathcal{H} \tilde{\boldsymbol{X}}
$$

of size $\left[N_{t} \times\left(\left(N_{p} \times N_{c}\right) \times W\right)\right]$. Here, the Hankelization operator $\mathcal{H}$ slides a window of size $[1 \times W]$ through channel $\tilde{X}_{c}$ of the zero-padded AC data and takes each block to be a row in the $c$-th column of the calibration matrix. This operation is similar to the construction of the calibration matrix in ESPIRiT [96].

We decompose $\boldsymbol{A}$ using an SVD

$$
\boldsymbol{A}=\boldsymbol{U} \boldsymbol{S} \boldsymbol{V}^{H},
$$


and consider $\boldsymbol{U}$ of size $\left[N_{t} \times N_{t}\right]$ as the orthonormal basis that consists of the Empirical Orthogonal Functions (EOFs) $U^{k}, 1 \leq k \leq N_{t}$. The principle components $\left(S V^{H}\right)_{k}$ are given by

$$
\left(S V^{H}\right)_{k}:=\sum_{l=1}^{W \cdot N_{c} \cdot N_{p}} S_{k}^{l}\left(V^{H}\right)_{l}=\lambda_{k}\left(V^{H}\right)_{k} .
$$

The expansion of $\boldsymbol{A}$, or $\tilde{\boldsymbol{X}}$, in the basis $\boldsymbol{U}$ then reads

$$
\tilde{X}_{c}^{t+j}=A_{t}^{c j}=\sum_{k=1}^{N_{t}} U_{t}^{k}\left(S V^{H}\right)_{k}^{c j},
$$

where $1 \leq t \leq N_{t}$ iterates through the temporal samples, $1 \leq c \leq\left(N_{c} \cdot N_{p}\right)$ through the channels and $0 \leq j<W$ is the index inside the sliding window. The EOFs can be considered as data-adaptive weighted moving averages of the original time series $\tilde{\boldsymbol{X}}$, with $\boldsymbol{V}$ being the data-adaptive filters [240, 250],

$$
\begin{gathered}
\boldsymbol{U} \boldsymbol{S}=\boldsymbol{A} \boldsymbol{V}, \\
U_{t}^{k}=\frac{1}{\lambda_{k}} \sum_{c=1}^{N_{p} \cdot N_{c}} \sum_{j=1}^{W} \tilde{X}_{c}^{t+j} V_{c j}^{k} .
\end{gathered}
$$

In fact, the columns of $\boldsymbol{V}$ can bee seen as a complete eigenfilter decomposition of the original time series [251]. These filters $V^{k}$ act as data-adaptive band-pass filters with a frequency bandwidth $\delta f_{\mathrm{B}}$ given by

$$
\delta f_{\mathrm{B}}=\frac{f_{\mathrm{s}}}{W^{\prime}}
$$

where $f_{\mathrm{s}}$ is the sampling rate $[252,253]$. Harris and Yuan showed in [250] for the univariate case that a periodic oscillation contained in the data lead to an even and odd filter. The application of these filters to the original time series constitutes for each oscillation one EOF which is in phase and one which is in quadrature to the original oscillation, respectively.

Vautard et al. [240] proposed another interpretation of the EOFs considering the minimization problem

$$
\left.\underset{\alpha}{\arg \min }\right|_{t, k} \sum_{c=1}^{N_{p} \cdot N_{c}} \sum_{j=1}^{W}\left\|\tilde{X}_{c}^{t+j}-\alpha\left(S V^{H}\right)_{k}^{c j}\right\|^{2} .
$$

The solution of Equation (7.15) is $\alpha=U_{t}{ }^{k}$, thus the EOFs can be obtained by a local least-squares fit of the $\mathrm{k}$-th principle component to the original time series. This locality, determined by the window size $W$, distinguishes SSA-FARY from classical 
PCA, which does not take the temporal past and future of a sample into account. In fact, PCA is a special case of SSA-FARY with $W=1$,

$$
\left.\boldsymbol{A}\right|_{W=1}=\mathcal{H} \tilde{\boldsymbol{X}}=\mathcal{H} \mathcal{Z} \boldsymbol{X}=\boldsymbol{X}^{T} .
$$

In conventional SSA, i.e. where no zero-padding is applied, the EOFs are of reduced length $N_{t}-W+1$. Thus, the exact correspondence in time is lost, which inhibits their use as self-gating signal. In contrast, the EOFs $U^{k}$ in SSA-FARY preserve the length $N_{t}$ of the original time series and can directly be used for selfgating, similar to the eigenvectors $U^{k}$ in PCA. In distinction to PCA, the EOFs in SSA(-FARY) capture temporal oscillations via oscillatory pairs [239]. In particular, if two consecutive eigenvalues are nearly equal, the two corresponding EOFs are nearly periodic with the same period and in quadrature [254], which is a consequence of the filtering property of SSA-FARY [250]. To ensure a proper separation the singular values of different EOF pairs should be distinct, which is called the strong separability condition [255] and usually fulfilled for our application.

These pairs can be seen as the data-adaptive equivalent to the sine-cosine pairs of Fourier analysis [256]. A single EOF pair might suffice for the analysis of nonlinear and inharmonic oscillations, as it automatically locates intermittent oscillatory regions. In contrast, classical spectral analysis would require a large amount of harmonics or subharmonics of the fundamental period [240, 257].

COMments The EOF $U^{k}$ is also the k-th left eigenvector of the $\left[N_{t} \times N_{t}\right]$ realsymmetric cross-covariance matrix

$$
\boldsymbol{C}=\boldsymbol{A} \boldsymbol{A}^{H} .
$$

Depending on the number of acquired spokes and partitions, computing the eigendecomposition of $\boldsymbol{C}$ is usually more efficient than computing the SVD of $\boldsymbol{A}$.

In contrast to the parameter-free PCA, for SSA we must define a window size $W$. To long-range correlations in time, $W$ should be large, which - as a trade-off results in a lower degree of statistical confidence [257]. Vautard et al. [240] showed that SSA can resolve oscillations best when the periods are shorter than the window size $W$. In our study, the window size $W \approx 3 \mathrm{~s}$ proofed to be a robust choice for most measurements, independently of the utilized sequence, and this value was chosen as the default. More information on the choice of the window size is given in the Methods and Discussion section.

The fundamental concept behind the use of a temporal window $W$ is Taken's delay embedding theorem [179], one of the backbones of chaotic dynamical system analysis. Instead of considering each temporal sample individually and isolated from other time points, so called time-delay coordinates are constructed by embedding the samples in a higher-dimensional space with embedding dimension $W$. Consequently, each time point is represented by a time-delay coordinate vector, 
which comprises not only the sample of the respective time but also its temporal past and future. It is therefore a natural choice to pick an odd value for $W$ in order to incorporate the same amount of past and future information.

Towards the beginning and the end of a time series this embedding can no longer be constructed due to lack of past or future samples, respectively. There are two strategies to overcome this limitation: 1.) Time-delay coordinates are constructed for the central $N_{t}-(W-1)$ samples only, which means that $W-1$ samples would be discarded from future processing. 2.) $(W-1) / 2$ samples are zero-padded on both ends of the time series, which comes at the expense of increased inaccuracy for the marginal samples of the time series. However, for the second approach no samples have to be discarded and through the symmetric zero-padding the timedelay coordinates remain in sync with the actual temporal evolution of the signal. In this manuscript, the second approach is used.

\subsubsection{Binning}

The myocardium shows different behavior for contraction and expansion, so usually the entire cardiac cycle is divided into multiple distinct bins to accurately resolve the temporal motion. For respiratory gating it is usually assumed that inspiration and expiration do not have to be distinguished [57, 143, 258]. However, various studies reveal that respiratory motion is heavily subject-dependent and exhibits a strong variability as well as hysteresis, which affects the global position of the myocardium [139, 259-261]. Hence, inspiration and expiration should be distinguished to properly resolve the effects of breathing motion on the heart.

Since SSA-FARY yields EOF quadrature pairs that capture the phase information of periodic oscillations, binning is straight-forward for both cardiac and respiratory motion: The phase portrait, i.e. the amplitude-amplitude scatter plot, of an EOF pair is divided into $N$ circular sectors with central angle $\phi=360^{\circ} / N$. The samples are then binned according to their respective circular sector, see Figure 7.1b. 


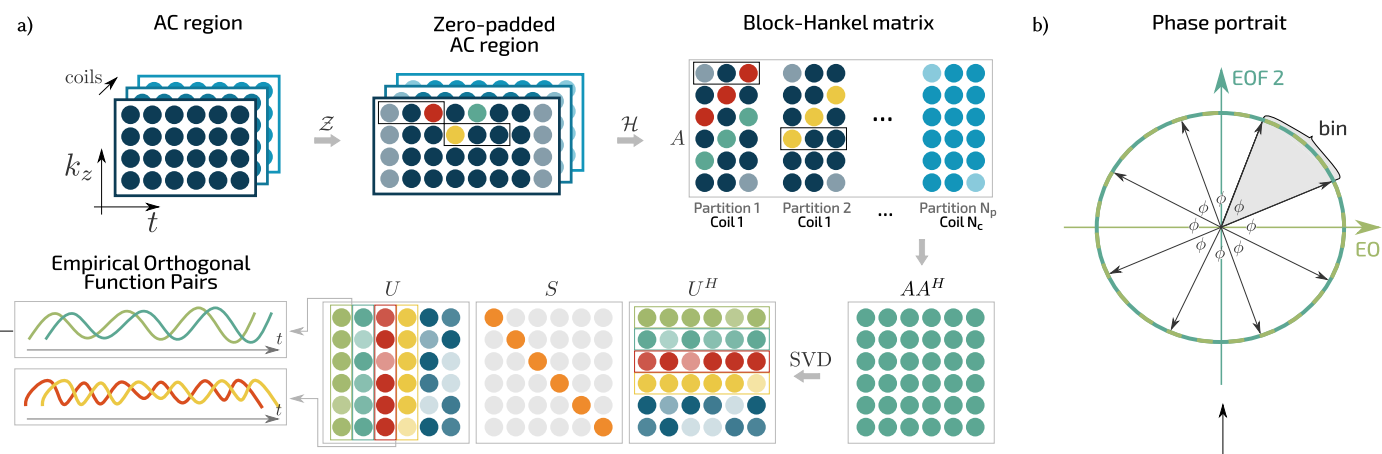

Figure 7.1.: (a) Schematic of SSA-FARY: The multi-channel AC data is zero-padded $\mathcal{Z}$ and Hankelized $\mathcal{H}$. Then the cross-correlation matrix $A A^{H}$ is calculated and decomposed using an eigenvalue or singular value decomposition. The left eigenvector $U$ contains the EOF quadrature pairs that represent the principle motion signals of the time series. (b) Binning: The phase portrait of an EOF quadrature pair is divided into $N$ circular sectors with equal central angle $\varphi=360^{\circ} / N$. The samples are then assigned to bins according to their respective circular sector.

\subsection{METHODS}

\subsubsection{Numerical Simulations}

We compare the capability of PCA and SSA-FARY in extracting and separating oscillatory signals in simple numerical simulations.

The signals we want to extract are two frequency-modulated sinusoids

$$
\begin{aligned}
& a(t)=A \sin \left(\varphi_{a}+\omega_{a} t+\phi_{a}(t)\right), \\
& b(t)=B \sin \left(\varphi_{b}+\omega_{b} t+\phi_{b}(t)\right),
\end{aligned}
$$

with

$$
\begin{gathered}
\phi_{a}(t)=\Phi \sin (2 \pi t / 2 T), \\
\phi_{b}(t)=\Phi \sin (2 \pi t / T),
\end{gathered}
$$

which account for frequency variations. To simulate various channels $i$, we use a weighted sum

$$
x_{i}(t)=\frac{i}{N_{c}} a(t)+\frac{\left(N_{c}+1-i\right)}{N_{c}} b(t), \quad 1 \leq i \leq N_{c} .
$$

To spoil the composite signals $x_{i}(t)$, we add Gaussian white noise $h_{\text {noise }}(t)$ with standard deviation $\sigma_{\text {noise, }}$ or an oscillatory spell from time $t_{1}$ to time $t_{2}$,

$$
h_{\text {spell }}(t)=C \sin \left(\varphi_{c}+\omega_{c} t\right), \quad t_{1} \leq t \leq t_{2},
$$

or an exponential trend

$$
h_{\text {trend }}(t)=D_{1} e^{\xi t}-D_{2}
$$


to all channels, which yields

$$
X_{i}(t)=x_{i}(t)+h(t) .
$$

We analyze the time-series $\boldsymbol{X}^{\text {noise }}, \boldsymbol{X}^{\text {spell }}$ and $\boldsymbol{X}^{\text {trend }}$ using PCA and SSA-FARY with window size $W$. Note that the aim of this numerical experiment is to demonstrate the general benefits of SSA-FARY over PCA for the analysis of time series, and not to simulate cardiac and respiratory motion in the most accurate way. We therefore did not include the modeling of a more complex frequency variability or motion signal shapes. More details on the simulation are provided in Section 7.8.

\subsubsection{In Vivo Experiments}

All measurements were performed on a Skyra $3 \mathrm{~T}$ scanner (Siemens Healthcare $\mathrm{GmbH}$, Erlangen, Germany) using 30 channels of a thorax and spine coil. Gradient delay correction was performed using $\operatorname{RING}[58,59]$. The AC data was corrected using the orthogonal projection with $N_{H}=5$. In the following, the AC data's real and imaginary part are treated as individual channels. The Field of View (FOV) in all experiments was $256 \times 256 \mathrm{~mm}^{2}$ at base resolution 192. All presented experiments were performed on volunteers with no known diseases, who gave written informed consent. All SOS measurements were performed on different volunteers. The study had received approval from the local ethics committee.

SEQUENCE DESIGN, AUTO-CALIBRATION AND RECONSTRUCTION We utilize a radial bSSFP sequence for the single-slice measurement, an RF-spoiled gradientecho sequence with randomized RF spoiling [90] for the SMS measurement and a radial bSSFP sequence with undersampling in $k_{z}$ direction [164] for the SOS measurement. To obtain maximum k-space coverage and thus improved image quality $[60,166]$, the projection angle $\varphi$ is increased in each shot about the seventh tiny golden angle $\varphi_{\mathrm{o}} \approx 23.6^{\circ}$ [170]. For the SMS and SOS measurements, the partitions are acquired in an interleaved fashion, i.e. one spoke is recorded for each partition before the next in-plane spoke of a partition is acquired.

For single-slice imaging, the central sample of all spokes is used for auto-calibration. For SMS, more AC data is available as not only a single sample but the central line along the $k_{z}\left(k_{x}=0, k_{y}=0\right)$ direction can be utilized.

Inspired by [164], we make use of variable-density $k_{z}$-undersampling for the SOS acquisition. From the total number of 14 partitions, the central 6 partitions are always acquired and the corresponding central line is used for auto-calibration. The remaining 8 partitions are undersampled by a factor of 4 . This center-dense sampling scheme does not only increase the temporal resolution of the AC data but also improves the image quality [262]. 
For self-gating with PCA and SSA-FARY as well as for imaging reconstruction we use BART [210]. Image reconstruction is performed using combined Parallel Imaging and Compressed Sensing (PICS) [14] applying the Alternating Direction Method of Multipliers (ADMM) [108] with in-plane wavelet-regularization on the spatial dimensions and Total Variation (TV) on the cardiac and respiratory dimension $[143,263]$. For SOS imaging, we additionally apply TV regularization in slice direction. The coil sensitivities for the single-slice and SMS measurements are generated using radial ENLIVE allowing two maps [41, 60, 96, 264]. To reduce the memory demand we allow only one map in the SOS reconstruction. We apply coil compression $[265,266]$ to reduce the number of coils to 13 for single-slice and SMS, and 10 for SOS imaging and perform the calibration of the sensitivities using a lower resolution.

The SSA-FARY gating signals is distributed into 25 cardiac and 9 respiratory bins for image reconstruction. Although not always necessary, we standardly perform an additional detrending of the EOFs using a moving average filter of length $L_{\text {avg }} \approx 3 W$. This further improves the binning accuracy of SSA-FARY by removing a possibly remaining residual trend.

In the spirit of reproducible research, code and data to reproduce the experiments are made available on Github. ${ }^{1}$

SINGLE-SLICE IMAGING We perform a 72 second free-breathing bSSFP scan $\left(\mathrm{TE} / \mathrm{TR}=1.90 / 3.80 \mathrm{~ms}\right.$, flip angle $\left.40^{\circ}\right)$ with slice-thickness $7 \mathrm{~mm}$ of the human heart in short-axis view and use the first 30 seconds of data for further analysis and image reconstruction. The full 72 second scan is used for a gridding reconstruction in Section 7.9.2. We furthermore conduct an ECG-triggered CINE bSSFP breathhold scan of the same slice $\left(\mathrm{TE} / \mathrm{TR}=1.52 / 3.04 \mathrm{~ms}\right.$, flip angle $57^{\circ}$, slice-thickness $7 \mathrm{~mm})$.

We compare the principal motion signals using PCA and SSA-FARY with window size $W=751$, which covers a period of about $3 \mathrm{~s}$.

SMS IMAGING We perform a 6o second free-breathing SMS RF-spoiled gradientecho scan $\left(\mathrm{TE} / \mathrm{TR}=1.79 / 2.90 \mathrm{~ms}\right.$, flip angle $\left.12^{\circ}\right)$ with three simultaneously acquired slices in short-axis view. The slice thickness is $5 \mathrm{~mm}$ and the slice gap 1o mm. We use SSA-FARY with window size $W=345$, which covers a period of about $3 \mathrm{~s}$. We perform a joint reconstruction of all slices using binning based on SSA-FARY. To evaluate the accuracy of SSA-FARY, we compare the SSA-FARY respiration quadrature-signals of the complete time series with the breathing pattern extracted from a pneumatic respiratory belt (Siemens Healthcare $\mathrm{GmbH}$, Erlangen, Germany) and a real-time reconstruction [6o, 62].

1 https://github.com/mrirecon/SSA-FARY 
SOS IMAGING We measure 8 volunteers and on each we perform a free-breathing three-minute radial SOS bSSFP scan (TE/TR $=1.90 / 3.80 \mathrm{~ms}$, flip angle $35^{\circ}$ ) with fourteen partitions in short-axis view and slice thickness $7 \mathrm{~mm}$. By default we use SSA-FARY with window size $W=91$, which covers a period of about $2.8 \mathrm{~s}$. For one volunteer the respiratory EOF pair revealed highly irregular breathing with occasional breath-holds of up to $12 \mathrm{~s}$ and for another volunteer the cardiac EOF pair showed a pronounced frequency variation. To improve the gating accuracy in these two cases, we determined another respiratory EOF pair using window size $W=51$ and another cardiac EOF pair using window size $W=21$, respectively.

To evaluate the precision of the cardiac gating with SSA-FARY, we analyze the SSA-FARY signal in comparison to the simultaneously acquired ECG trigger using a Python 3 script. Therefore, we define a synthetic trigger point when the phase related to the orthogonal cardiac SSA-FARY quadrature pair experiences a zerophase crossing. Since the global phase-offset of the quadrature pair is arbitrary, we correct the synthetic SSA-FARY trigger by a constant shift using the average distance to the ECG trigger. We then compute the standard deviation $\sigma_{\text {trig }}$ and standard error of the corrected SSA-FARY trigger to the ECG trigger.

Moreover, we acquire the same slices using a conventional ECG-triggered breathhold CINE single-slice stack bSSFP measurement with Cartesian read-out (TE/TR $=1.52 / 3.04 \mathrm{~ms}$, flip angles depending on the Specific Absorption Rate (SAR) limits between $47^{\circ}$ and $62^{\circ}$ ) and cardiac bin size $T_{\text {bin }} \approx 48 \mathrm{~ms}$. The patient dependent measurement time is around $6-8 \mathrm{~min}$. We compare the end-diastolic and endsystolic left-ventricular blood-pool area of a mid-ventricular slice to the SSA-FARY based reconstruction using ImageJ.

\section{$7 \cdot 4$ RESULTS}

NUMERICAL SIMULATIONS Figure 7.2 depicts the results of the numerical simulations. PCA is able to extract, at least essentially, the shape of the two oscillations $a(t)$ and $b(t)$ from $\boldsymbol{X}^{\text {noise }}$ and $\boldsymbol{X}^{\text {spell }}$. However, both the noise and the oscillation spell are still evident in the resulting eigenvectors and corrupt the signal. PCA fails to produce a useful result for $\boldsymbol{X}^{\text {trend }}$. While oscillation $a(t)$ is present in two principle eigenvectors, oscillation $b(t)$ is not distinctly separated in any of the eigenvectors.

In contrast, SSA-FARY extracts the oscillation signals with almost no spoiling residuals in all three investigated cases. Only at the borders deviations from the ideal signal can be observed. Notably, SSA-FARY does not only yield a one-dimensional signal of the temporal evolution of an oscillation, but preserves the phase information by quadrature pairs, as can be appreciated from the amplitude-amplitude plots. The two EOFs corresponding to the same pair have very similar singular values. In the scree plot of Figure 7.2 (b) and (c), the first plateau corresponds to EOF 1 

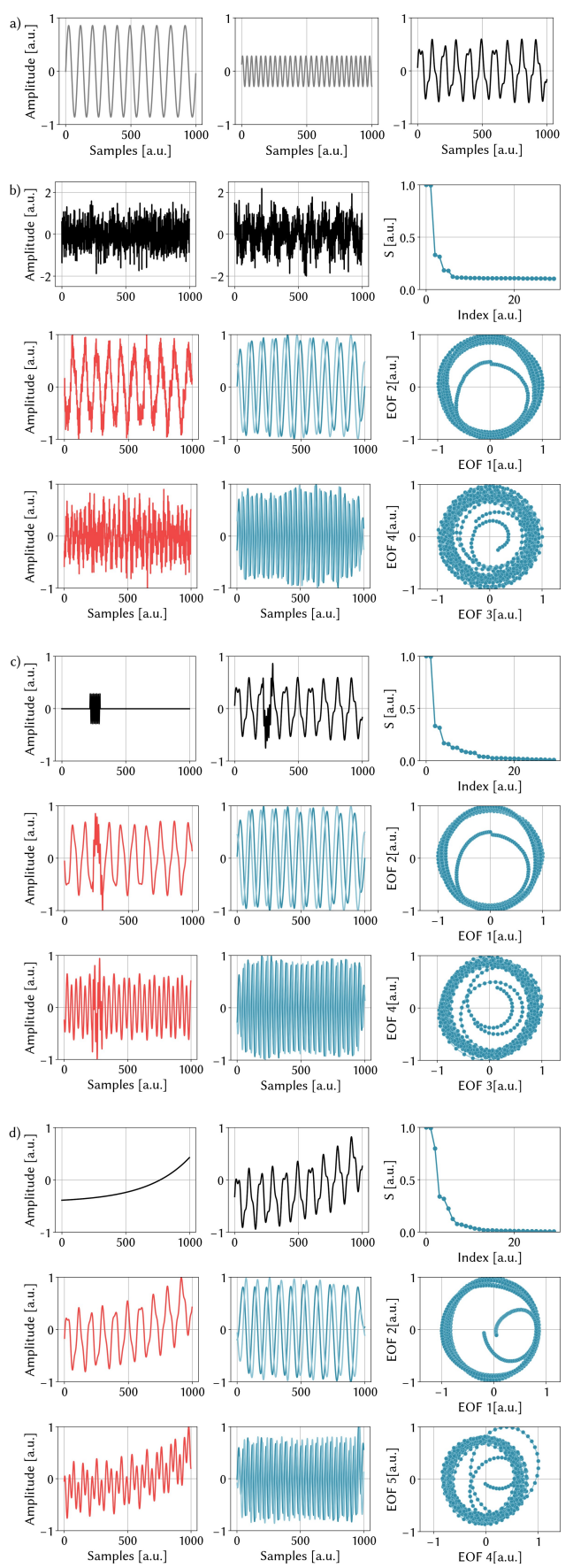

Figure 7.2.: Comparison of PCA eigenvectors and SSA-FARY EOFs. (a) Frequencymodulated sinusoidal oscillations $(a(t)(l e f t), b(t)$ (center)) used for the numerical simulations and weighted composite signal (right). (b), (c), (d) (Black): Different additional signals (left) - (b) noise, (c) oscillatory spell, (d) exponential trend - added to the composite signal of (a) and a selected channel of the resulting signal $X_{i}(t)$ (right). (Red): First two eigenvectors of the PCA. (Blue): SSA-FARY singular values $S$ (scree plot) and EOF pairs (amplitude-time plot and amplitude-amplitude scatter plot). All plots are normalized. 
and 2, and the second plateau belongs to EOF 3 and 4. In Figure 7.2d, the first two plateaus correspond to EOF 1 and 2, and to EOF 4 and 5. Hence, SSA-FARY does not mix the trend into the oscillations but creates an additional EOF to account for it. $^{2}$

SINGLE-SLICE IMAGING For each coil, Figure 7.3 depicts the DC component of 100 consecutive spokes before and after the data correction using the orthogonal projection. Before correction some coils exhibit pronounced oscillations with approximately 15 samples per period. As we have used the seventh tiny golden angle $\left(\varphi_{\mathrm{o}} \approx 23.6^{\circ}\right)$ the oscillations period of 15 samples corresponds to $\varphi^{15}=15 \cdot \varphi_{\mathrm{o}} \approx 354^{\circ}$ (see Equation (7.1)). Hence, the oscillation period in the AC data is linked to the period of the projection angle. By removing this frequency and the higher-order harmonics these oscillations can be completely eliminated.

a)

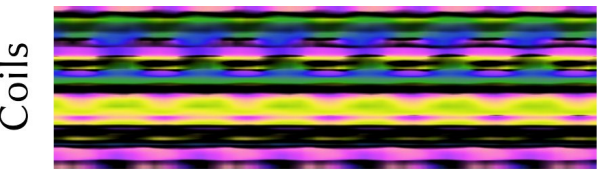

Time $\rightarrow$ b)

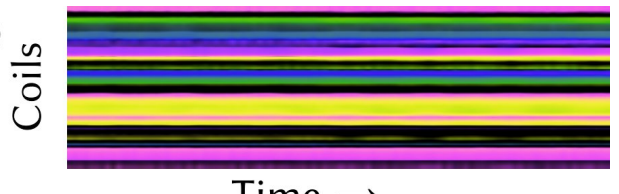

Figure 7.3.: Snippet of the amplitude plot with color-coded phase of the DC samples used for auto-calibration of the single-slice reconstruction before (a) and after (b) the data correction using the orthogonal projection. The period length of the oscillations in (a) corresponds to 15 samples.

Figure $7 \cdot 4$ shows the self-gating signals generated with PCA and SSA-FARY. In SSA-FARY, the first two EOFs represent respiratory motion and the third and fourth EOF cardiac motion. The EOFs of the pairs are in quadrature, respectively. Both the cardiac and the respiratory phases are well separated. In contrast, PCA cannot fully extract and separate the signals as respiratory and cardiac motion are superposed and heavily spoiled by noise.

Figure 7.4C shows six representative images of the SSA-FARY-gated reconstruction. Depicted are from bottom to top the end-systolic, an intermittent and the end-diastolic frame for end-expiration and end-inspiration, respectively. For comparison, we also show the result of the ECG-triggered CINE breath-hold scan.

In Section 7.9.2 we present the results of a conventional gridding reconstruction of the full 72 second measurement gated with SSA-FARY. ${ }^{3}$

2 In Figures 7.7 to 7.9 we provide results for simulations with different noise variance and trend and spell amplitudes, as well as a different frequency variation. More information on these figures is provided in Section 7.9.1.

3 Figure 7.10 shows the SSA-FARY and PCA gating signals of the 72 second scan. Moreover, a gridding reconstruction for three different cardiac phases is depicted and the results of a breath-hold and ECGgated CINE scan are shown for comparison. 
In Section 7.9.3 we present a similar experiment with an RF-spoiled gradientecho sequence. 4

SMS IMAGING Figure 7.5a shows 5 out of 25 cardiac phases for all three slices in end-expiration. The different systolic and diastolic phases are well resolved.

The background of Figure $7 \cdot 5 \mathrm{~b}$ shows the temporal evolution of a line extracted from an SMS-NLINV real-time reconstruction of the full time series. The line was placed in slice two in vertical direction such that the actual motion of the diaphragm can be observed. This diaphragmatic motion is linearly related to the translation of the heart during breathing [267]. On top of the background image, we have plotted the respiratory EOF quadrature pair obtained from SSA-FARY as well as the signal provided by the respiratory belt. For the purpose of comparison, the motion signals were scaled according to the amplitude of the diaphragm motion in the background image.

One of the EOFs (light blue) coincides very well both in amplitude and phase with the temporal evolution of the diaphragm. This is in line with the filtering interpretation of SSA-FARY, which states that one EOF of the quadrature pair is in phase with the related underlying motion, whereas the other EOF (dark blue) constitutes the quadrature signal. The in-phase EOF is furthermore in good agreement with the motion signal provided by the respiratory belt.

The presented volunteer shows a largely periodic breathing pattern. In Section 7.9.4 we present the results of the same experiment on another volunteer, which happend to exhibit a highly irregular respiration pattern during measurement. ${ }^{5}$

SOS IMAGING Figure 7.6a depicts a zoomed view on EOFs representing respiratory and cardiac motion for different window sizes. For window size $W=31$, which - considering the undersampling scheme - covers a period of $\approx 1.0 \mathrm{~s}$, respiratory and cardiac motion are not fully separated and appear superposed in one EOF for respiratory and one EOF for cardiac motion. In contrast, for the proposed window size $W=91(\approx 2.8 \mathrm{~s})$ the motion signals are well separated. Then again, for $W=151(\approx 4.5 \mathrm{~s})$ a signal loss can be observed in the cardiac EOFs. Figure $7.6 \mathrm{~b}$ shows one respiratory EOF and one cardiac EOF for the windows $W=81, W=91$ and $W=101$, covering periods from $2.5 \mathrm{~s}$ to $3.0 \mathrm{~s}$. All signals are in good agreement. The corresponding pairing-components of the EOFs show similar behavior and are

4 Figure 7.11 shows the effect of the proposed correction on the AC region. Figure 7.12 shows the self-gating signals determined with SSA-FARY and PCA. Furthermore, six representative frames of the SSA-FARY-gated reconstruction for different respiratory and cardiac motion states are depicted.

5 In Figure 7.13 we provide results of the same experiment conducted on a different volunteer. We depict 5 cardiac phases for all three slices, as well as a comparison between the SSA-FARY respiratory self-gating signal, the respiratory belt and the diaphragm motion extracted from a real-time reconstruction. 

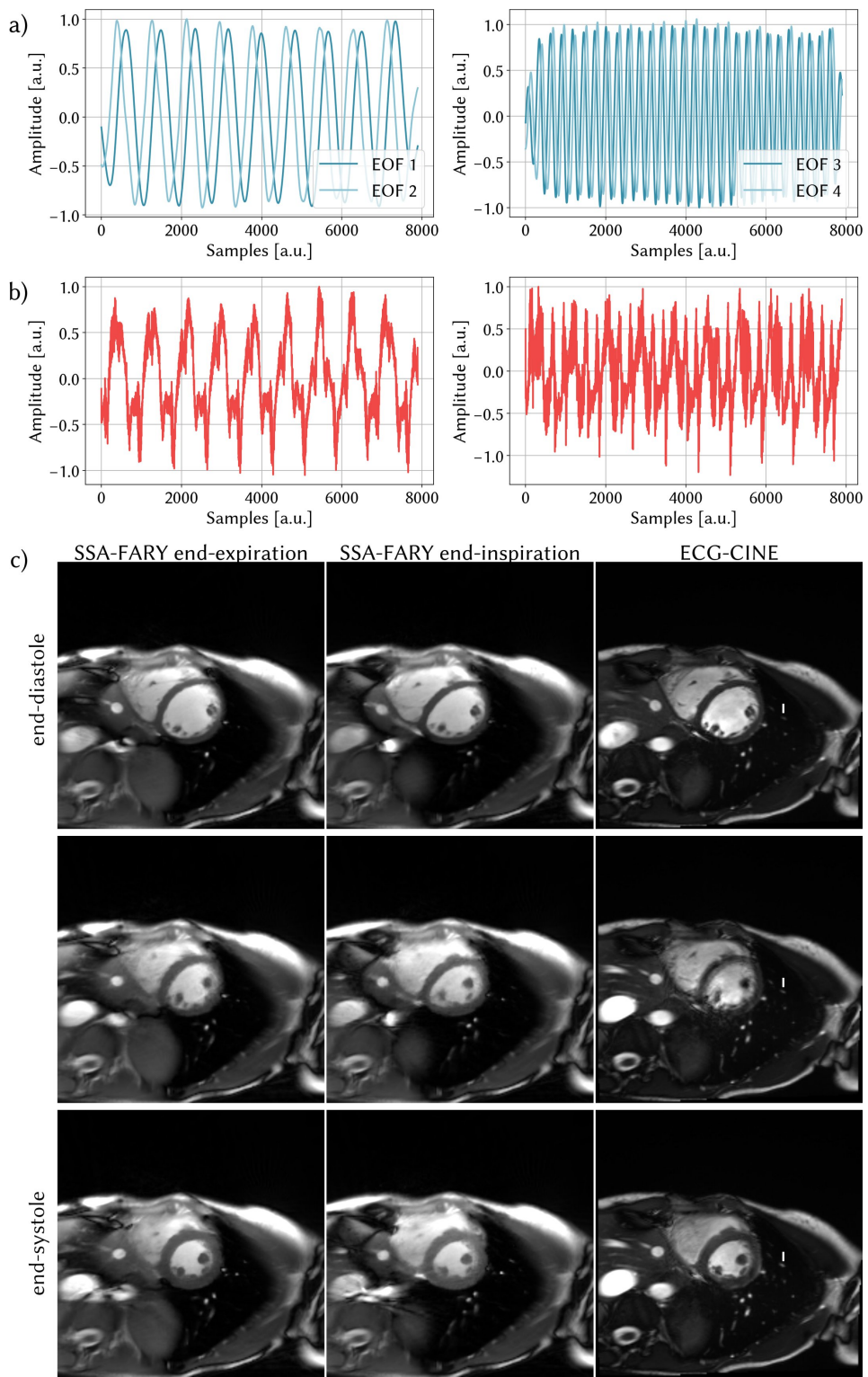

Figure 7.4:: Self-gating signals and PICS reconstruction of the human heart from a single-slice radial bSSFP acquisition in comparison to a conventional CINE scan. In (a) the two quadrature EOFs of SSA-FARY are plotted against time for the respiratory (left) and cardiac (right) motion, respectively. In (b) the first two eigenfunctions of the PCA are depicted. In both, a superposition of the cardiac and respiratory motion spoiled by additional noise can be perceived. For (c) the self-gating signal of (a) was utilized. It shows 6 representative frames of the PICS reconstruction using SSA-FARY corresponding to three cardiac phases from end-systole to end-diastole at end-expiration and end-inspiration. For comparison, we also show the vendor images of an ECG-triggered CINE breath-hold scan. 
a)

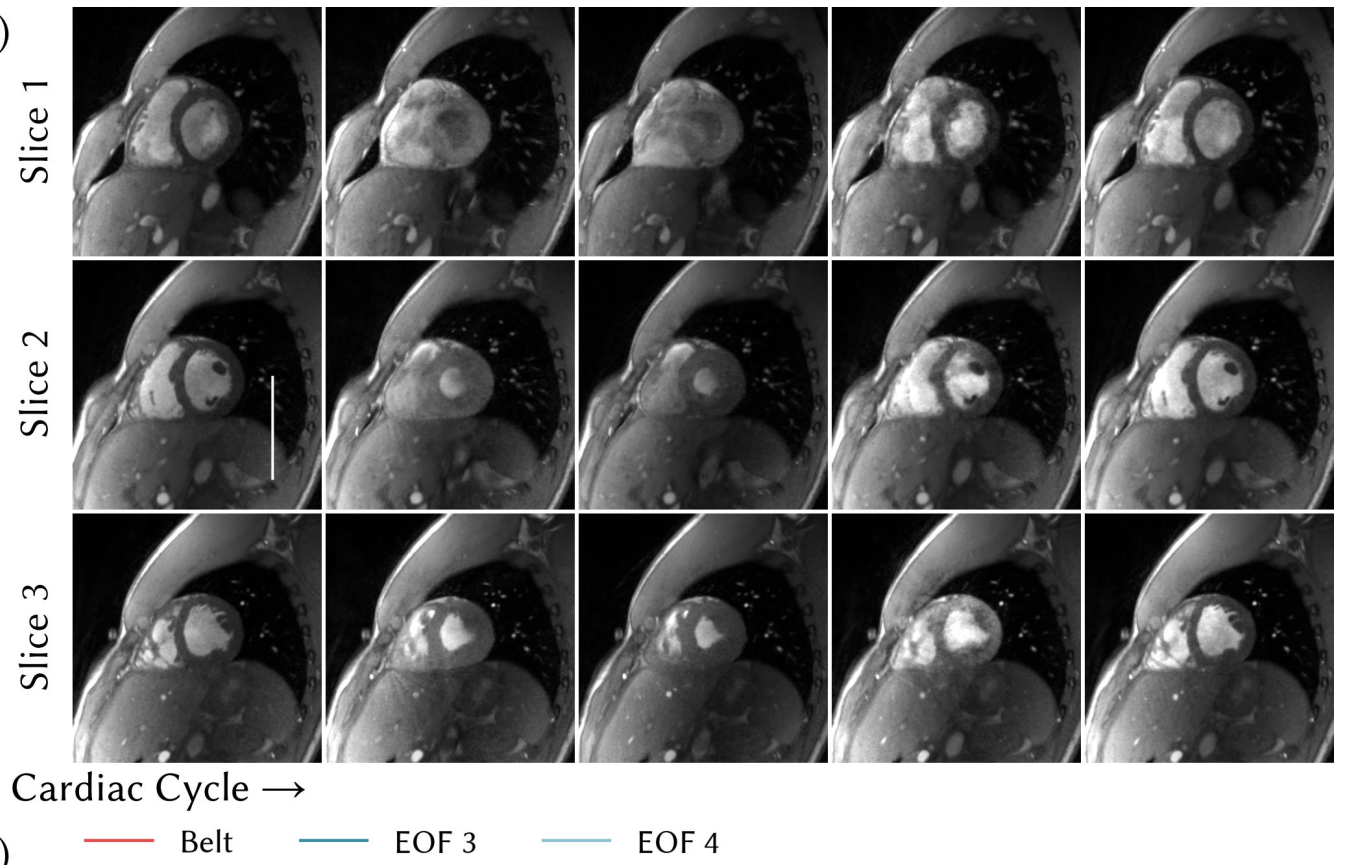

b)

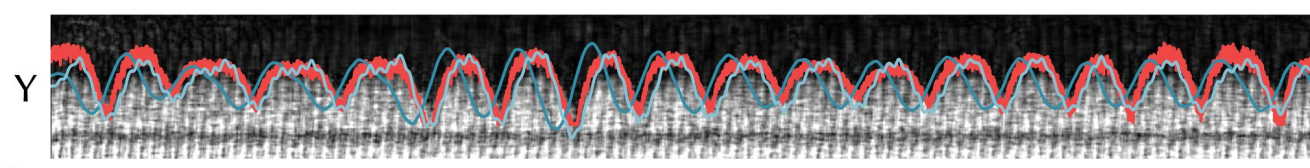

Time $\rightarrow$

Figure 7.5.: Images of the human heart reconstructed using PICS and the SSA-FARY self-gating signal from a radial SMS gradient-echo acquisition. (a) shows 5 out of 25 cardiac phases in end-expiration for each of the three slices. The background of (b) is extracted from an SMS-NLINV real-time reconstruction of the entire time series and shows the temporal evolution of a vertical line (highlighted in white), placed on the diaphragm in slice two. On top, the respiratory self-gating EOF quadrature pair of SSA-FARY and the signal extracted from the respiratory belt is plotted. The amplitudes of the motion signals are scaled for the purpose of comparison. 
therefore not depicted. Figure 7.6c presents end-diastolic and end-systolic frames for three out of fourteen slices in end-expiration. All respiratory and cardiac states are well separated, the cardiac wall and the diaphragm are sharply resolved. Note, however, that some slices at the fringe of the slab have low signal intensity due to an unoptimized excitation profile. The image quality is comparable to the CINE reconstruction Figure $7.6 \mathrm{~d}$, although the latter tends to be sharper. Due to the higher flip-angle, which is restricted by SAR limitations in volumetric sequences, the CINE images possess a better blood myocardium contrast.

For the different volunteers, Table 7.1 shows the average heart-rate $\bar{f}_{\text {heart }}$, its standard deviation $\sigma_{\text {heart }}$, the standard deviation $\sigma_{\text {trig }}$ of the synthetic SSA-FARY trigger to the ECG trigger and the end-diastolic and end-systolic left-ventricular blood-pool area of a mid-ventricular slice for CINE and SSA-FARY.

For imaging on a $3 \mathrm{~T}$ system an insufficient shim can lead to banding artifacts. Measurements with bandings affecting the heart could not immediately be noticed and repeated as the reconstruction was performed offline. Therefore, these measurements were discarded for which the analysis of one volunteer is omitted.

The observed average heart rates range from $0.91 \mathrm{~Hz}(54.60 \mathrm{bpm})$ to $1.38 \mathrm{~Hz}$ (82.20 bpm) with different heart rate variabilities. For the given SOS acquisition with 14 partitions (6 AC lines and undersampling factor of 4 ), the temporal resolution of the SSA-FARY trigger is $30.4 \mathrm{~ms}$. The standard deviation of the SSA-FARY trigger to the ECG trigger, $\sigma_{\text {trig, }}$, is of similar size. Hence, the SSA-FARY trigger is in good agreement with the ECG signal and also matches the temporal resolution of the ECG-CINE acquisition.

The areas of the chosen mid-ventricular slices are comparable for ECG-CINE and SSA-FARY, particularly for end-diastole the difference lies mostly in the lower single-digit percent range, whereas a larger uncertainty can be observed for endsystole.

Volunteer V6 exhibits a highly erratic breathing pattern and volunteer $\mathrm{V}_{7}$ possesses a strongly irregular heartbeat and furthermore unintentionally yawned three times during the measurement. Still, SSA-FARY can provide satisfying results as Table 7.1 and Figures 7.14 and 7.15 show. $^{6}$

For all in vivo experiments, we have attached representative movies as Supplementary Material. ${ }^{7}$

6 Figures 7.14 and 7.15 display the SSA-FARY gating signal, representative frames of the SSA-FARYbased image reconstruction and the corresponding CINE reconstructions for volunteers V6 and $V_{7}$. More information on the figures is provided in Section 7.9.5.

7 The files Mov1-14 of the Supplementary Material show representative movies of all in vivo reconstructions. More information on the movies is provided in Section 7.9.6. 
Table 7.1.: Average heart rate $\bar{f}_{\text {heart }}$ and corresponding standard deviation $\sigma_{\text {heart }}$, standard deviation of SSA-FARY from ECG trigger $\sigma_{\text {trig. }}$. End-systolic and end-diastolic left-ventricular blood-pool area of a mid-ventricular slice for ECG-CINE and SSAFARY and corresponding error. * denotes the volunteer with highly erratic respiration, ** denotes the volunteer who yawned.

\begin{tabular}{|c|c|c|c|c|c|c|c|c|c|}
\hline \multirow[t]{2}{*}{ Volunteer } & \multirow[t]{2}{*}{$\bar{f}_{\text {heart }}[\mathrm{Hz}]$} & \multirow[t]{2}{*}{$\sigma_{\text {heart }}[\mathrm{Hz}]$} & \multirow[t]{2}{*}{$\sigma_{\text {trig }}[\mathrm{ms}]$} & \multicolumn{3}{|c|}{ Diastole $\left[\mathrm{mm}^{2}\right]$} & \multicolumn{3}{|c|}{ Systole $\left[\mathrm{mm}^{2}\right]$} \\
\hline & & & & $\mathrm{ECG}$ & SSA-FARY & Err. [\%] & ECG & SSA-FARY & Err. [\%] \\
\hline $\mathrm{V}_{1}$ & $0.910(4)$ & $0.057(3)$ & $23(1)$ & 2254 & 2222 & 1.4 & 1068 & 1017 & 4.8 \\
\hline $\mathrm{V}_{2}$ & $1.380(3)$ & $0.0541(2)$ & $14 \cdot 7(7)$ & 1536 & 1647 & 7.2 & 573 & 706 & 23.2 \\
\hline $\mathrm{V}_{3}$ & $0.979(2)$ & $0.0245(1)$ & $28(2)$ & 1911 & 2161 & 13.1 & 1072 & 1168 & 11.0 \\
\hline $\mathrm{V}_{4}$ & $1.245(3)$ & $0.045(2)$ & $23(1)$ & 1998 & 1956 & 2.1 & 866 & 961 & 11.0 \\
\hline $\mathrm{V}_{5}$ & $0.955(4)$ & $0.056(3)$ & $19(1)$ & 2348 & 2380 & 1.4 & 1005 & 1179 & $17 \cdot 3$ \\
\hline $\mathrm{V} 6^{*}$ & $1.195(7)$ & $0.103(5)$ & $34(2)$ & 2244 & 2220 & 1.1 & 1351 & 1407 & 4.1 \\
\hline $\mathrm{V}_{7}^{* *}$ & $1.08(1)$ & $0.129(7)$ & $27(1)$ & 3398 & 3314 & 2.5 & 2060 & 1930 & 6.3 \\
\hline
\end{tabular}


a)

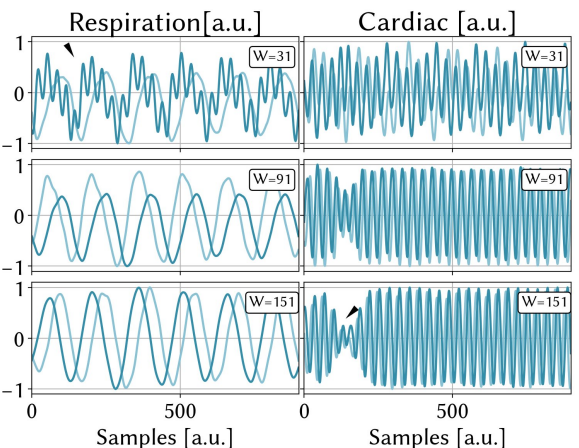

b)

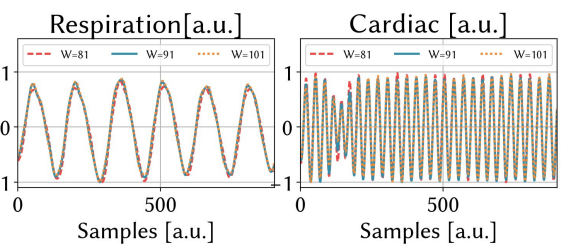

c)

SSA-FARY

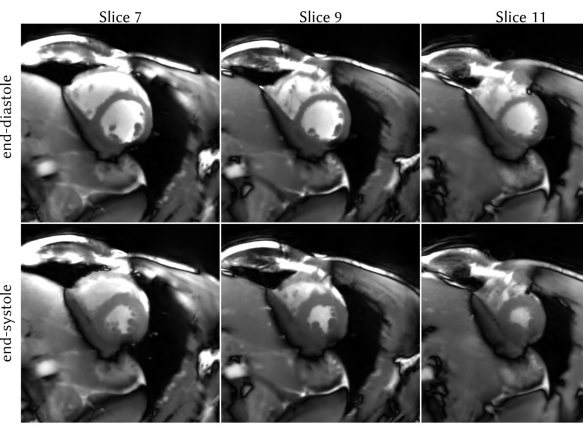

d) ECG-CINE

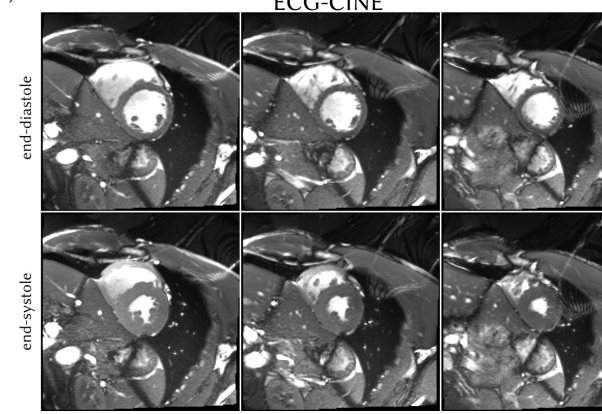

Figure 7.6.: SSA-FARY self-gating signals for different window sizes from a SOS bSSFP acquisition and representative slices from a $3 \mathrm{D}$ PICS reconstructions for volunteer $\mathrm{V}_{5}$ in comparison to a conventional CINE scan. (a) A zoomed view on normalized EOF pairs representing respiratory and cardiac motion are depicted for window sizes $W=31, W=91$ and $W=151$. The black arrows indicate signal mixing (top-left) and signal loss (bottom-right). (b) For window sizes $W=81, W=91$ and $W=101$, one EOF representing respiratory motion and one EOF representing cardiac motion are plotted in a zoomed view on top of each other. (c) shows three out of fourteen slices of an enddiastolic and end-systolic human heart after expiration. (d) depicts the corresponding vendor images for the ECG-triggered CINE breath-hold measurement. 


\section{$7 \cdot 5$ DISCUSSION}

We introduced a novel dimensionality reduction method dubbed "SSA-FARY", which is based on Singular Spectrum Analysis and showed that the proposed technique can successfully recover the cardiac and respiratory signal from the AC data of single-slice, SMS and SOS MRI measurements. Moreover, we have proposed an extended orthogonal projection to correct for system imperfections in the AC data.

AC CORRECTION The reasons for the oscillations in the AC data are many fold and according to our experience cannot be eliminated completely by techniques that correct for trajectory errors only [196, 198]. Particularly for bSSFP sequences, eddy current related dephasing of spins additionally compromises the data [168, 268], although this effect should be rather small for the tiny golden angle [170].

Zhang et al. [177] find this to be particularly problematic for $3 \mathrm{D}$ bSSFP imaging on a $3^{\mathrm{T}}$ system and propose to eliminate these measurement errors by averaging over the central five samples of each spoke. However, we found this to produce even more oscillations in the $\mathrm{AC}$ data, which requires additional filtering. In contrast, the orthogonal projection used here can remove most of the signal perturbations directly. For constant increments of the projection angle, the correction can be thought of as a set of sharp band-stop or notch filters corresponding to higherorder harmonics of a base frequency, which can be calculated from the increment of the projection angle.

Strictly speaking, the AC correction is not mandatory for SSA-FARY as without filtering these spurious oscillations appear as additional EOFs. However, as these oscillations usually manifest distinctly in the AC region, the corresponding EOFs often possess the highest singular values. Consequently, they dominate the result of Equation (7.15) which reduces the significance and accuracy of the components of interest, i.e. cardiac and respiratory motion, and complicates the analysis. This can be easily avoided by using the proposed orthogonal projection, which corrects for first-order system imperfections.

Alternatively, advanced techniques to measure the trajectory error [190, 269] or higher-order system imperfection corrections could be utilized to account for the oscillations in the AC region [192, 195]. Still, these approaches require additional hardware and/or sequence modifications, which limits their accessibility.

SSA-FARY The presented dimensionality reduction technique for time-series SSAFARY can be considered a PCA applied to a time-delayed embedding of the AC data to exploit the locally low-rankness of dynamic time series. The Block-Hankel matrix $\boldsymbol{A}$ (Equation (7.8)) consists of shifted segments of the original time series. Its covariance matrix $C_{t}^{t^{\prime}}=A_{t}\left(A^{H}\right)^{t^{\prime}}$ (Equation (7.17)) is an array of scalar products specifying the correlation of all pairs of multi-channel segments in the embedding 
space. The considerable redundancy in the correlations of the segments in the presence of (quasi) repetitive oscillations, e.g. cardiac and respiratory motion, causes $C$ to have low-rank. By exploiting not only spatial but also these temporal correlations, SSA-FARY performs better in recognizing temporal patterns than classical PCA and allows the separation of trend, oscillations and noise from the signal. This was successfully demonstrated on numerical simulations of superposed and spoiled sinusoidal time series. In the actual in vivo measurements the respiratory and cardiac motion could be detected and clearly separated for all investigated sequence types.

To yield comparable results, methods like classical PCA must be combined with various pre- and post-processing techniques such as coil-selection or coil-clustering [236], (iterative) band-pass filtering [258] and signal smoothing [143], which - especially for small AC regions - may be unstable and demand further manual tuning. By contrast, in SSA-FARY these steps are implicitly integrated and therefore surplus to requirement. Moreover, since SSA-FARY preserves phase information by producing quadrature pairs it allows for direct binning, which also renders the otherwise mandatory peak detection obsolete [52].

We demonstrated the high quality of the SSA-FARY gating signals by comparison with a real-time reconstruction, a respiratory belt and ECG triggers. Moreover, at free breathing and at a considerably lower acquisition time SSA-FARY achieved a reconstruction quality that comes close to the results of the ECG-triggered CINE breath-hold scans. The analyzed left-ventricular blood-pool area of SSA-FARY reconstructions mostly corresponds well to the CINE results for end-diastole, whereas larger uncertainties were found for end-systole. Here, because the left-ventricular blood-pool area is significantly decreased compared to end-diastolic states, any deviation will result in larger relative errors. One reason might be that due to the high acceleration factor the short end-systolic phase is not perfectly resolved. In addition, some discrepancy between a breath-hold scan and a gated free-breathing scan is expected, since it cannot be guaranteed that the selected respiratory bins exactly matches the anatomical state of the breath-hold scan.

As the aim of this manuscript was the introduction of the self-gating technique, we did not fully optimize the sequence and reconstruction parameters, particularly the number of cardiac and respiratory bins, the regularization values of the ADMM and the undersampling scheme of the SOS sequence. The parameter tuning and the setup of a clinically applicable protocol is left for future investigations.

In single-slice imaging we only use a single sample per time step for autocalibration and despite the proportionally large window size, which reduces statistical significance, SSA-FARY yields reliable results. Still, SSA-FARY tends to be more resilient when multiple partitions are used and thus more AC data is available, as in SMS or SOS experiments, or when more samples relative to the window size are used for auto-calibration. For SMS or SOS imaging a bSSFP sequence is 
recommended since for RF-spoiled gradient-echo imaging a loss of contrast in systolic phases can occur due to pre-saturated blood flowing in from other slices, see Figure 7.5. Note, however, that bSSFP sequences suffer from SAR limitations due to the increased flip-angle of the RF pulse and are prone to banding artifacts when no proper shimming is conducted.

Due to the zero-padding operation, Equation (7.7), and the subsequent Hankelization, Equation (7.8), the first and last samples in the SSA-FARY EOFs suffer from slight approximation errors. Still, for all presented experiments and analysis we did not discard these samples.

Occasionally, the trend was not completely separated from the EOFs for cardiac and respiratory motion, which we fixed by standardly using a moving average filter. The reason for the incomplete separation of trend can be understood by considering Equation (7.14), which relates the window size $W$ to the frequency bandwidth $\delta f_{\mathrm{B}}$ of the eigenfilters $\boldsymbol{V}$ generating the EOFs $\boldsymbol{U}$. Our default choice of window size and sampling rate corresponds to $\delta f_{\mathrm{B}} \approx 0.35 \mathrm{~Hz}$ for all measurements and sequences. If we choose $W$ too small, the EOFs capture a wider range of frequencies, which can result in a mixing of trend and oscillations. Similarly, if the respiration frequency (usually $f_{\text {resp }} \approx 0.3 \mathrm{~Hz}$ ) and cardiac frequency (usually $f_{\text {resp }} \approx 1.0 \mathrm{~Hz}$ ) happen to be spectrally close to one another, a very small window and the corresponding large frequency pass-band of the filters can hinder a proper separation, as it is the case in Figure $7.6 \mathrm{a}, W=31$. Then again, if we choose a very large $W$, the frequency response $f_{B}$ of the filters can be too narrow. Thus, possible frequency variations in the cardiac and respiratory motion can no longer be captured by a single (band-limited) EOF, which results in signal voids and/or the generation of additional EOFs representing higher order harmonics. Detecting these related EOFs corresponds to the 'Grouping' step of conventional SSA [270]. In the cardiac signal of Figure $7.6 \mathrm{a}$ such a signal void caused by a cardiac frequency variation of $\approx 0.3 \mathrm{~Hz}$ can be observed for $W=151$, whereas the signal can still be adequately captured with $W=91$. As a summary, we found $\delta f_{\mathrm{B}} \approx 0.35 \mathrm{~Hz}$ to be a robust choice and we propose to choose the window size accordingly using Equation (7.14).

The computational bottle-neck of SSA-FARY is the SVD of $C$ of size $\left[N_{t} \times N_{t}\right]$. Especially for single-slice imaging, the window size $W$ required to obtain $\delta f_{\mathrm{B}} \approx 0.35 \mathrm{~Hz}$ and the corresponding number of AC samples $N_{t}$ to obtain good results is relatively large. Hence, the decomposition of $C$ is rather time-consuming. Nevertheless, there are approaches to significantly speed up the decomposition stage [271, 272].

SSA-FARY reliably detected the EOF pairs corresponding to cardiac and respiratory motion, which for RF-spoiled gradient echo measurements usually possess the highest singular values. It is, however, not determined that the two largest components belong to the cardiac or the respiratory motion. For bSSFP sequences, which 
are generally more prone to system imperfections, we frequently found other components such as trends to have high singular values, too. Although for this study the components used for gating were chosen by visual inspection of the EOFs, it is fairly simple to automatize the assignment using a frequency analysis.

If one finds the separation of SSA-FARY to work insufficiently, a variation of the window size usually helps to recover a suitable gating result. Still, for patients with strong cardiac or respiratory frequency variations, highly non-periodic respiratory motion or arrhythmia the results of the presented SSA-FARY method might still be insufficient. In this case various promising extensions of SSA(-FARY) exist to improve the results, e.g. 'Nonlinear Laplacian Spectral Analysis', 'Sliding SSA', 'Oblique SSA', 'Nested SSA' and their combinations [255, 273, 274].

OUTLOOK The property of SSA-FARY to not only reliably separate cardiac and respiratory motion but also to extract the trend in data, suggests further applications. In dynamic contrast-enhanced MRI, SSA-FARY could replace spline-fitting [143] for separating motion and contrast enhancement. Furthermore, preliminary results suggest that the $T_{1}$ decay in inversion recovery sequences is detected as an individual component and separated from the motion signals, which would enable the simultaneous reconstruction of parameter maps and self-gated anatomical motion [175].

Last but not least we want to mention that the zero-padding approach used in SSA-FARY is not limited to self-gated MRI but turns multi-variate SSA into a general dimensionality reduction method, which might be useful for many other problems related to time-series analysis.

\subsection{CONCLUSION}

We have introduced a novel SSA-based dimensionality reduction method called SSA-FARY. Its intuitive approach, the easy implementation and its capability to separate cardiac and respiratory motion as well as trend makes it a promising approach for MRI self-gating, particularly when it is combined with efficient data acquisition schemes and a state-of-the-art image reconstruction technique.

\subsection{ACKNOWLEDGEMENTS}

The orthogonality projection to correct the AC data was originally conceived by Dr. Anthony G. Christodoulou (Biomedical Imaging Research Institute, Cedars-Sinai Medical Center). We extended it by including the higher-order harmonics.

Supported by the DZHK (German Centre for Cardiovascular Research), the Physics-to-Medicine Initiative Göttingen (LM der Niedersächsischen Vorab), the DFG (German Research Foundation) under grant UE 189/1-1, and the Studiens- 
tiftung des deutschen Volkes. We gratefully acknowledge the support of the NVIDIA Corporation with the donation of one NVIDIA TITAN Xp GPU for this research.

\subsection{APPENDIX: DETAILS FOR NUMERICAL SIMULATIONS}

Here we present the values for the variables used in the numerical simulation (Figure 7.2). The total duration $T=800$ [a.u.] consists of 1000 discrete samples. The other parameters can be found in Table 7.2.

Table 7.2.: Variables and values for the numerical simulation.

\begin{tabular}{cc}
\hline Variable & Value \\
\hline$N_{c}$ & 30 \\
$A$ & 3 \\
$\phi_{a}$ & 0 \\
$\omega_{a}$ & $\frac{2 \pi}{80}$ \\
$B$ & 1 \\
$\phi_{b}$ & 0.5 \\
$\omega_{b}$ & $\frac{2 \pi}{30}$ \\
$\sigma_{\text {noise }}$ & 2 \\
$C$ & 1 \\
$\phi_{c}$ & 0 \\
$\omega_{c}$ & $\frac{2 \pi}{10}$ \\
{$\left[t_{1}, t_{2}\right]$} & {$[220,300]$} \\
$D_{1}$ & 0.15 \\
$D_{2}$ & 1.5 \\
$\xi$ & $3 \cdot 75 \cdot 10^{-3}$ \\
$W$ & 101 \\
$\Phi$ & 2 \\
\hline
\end{tabular}

7.9 SUPPLEMENTARY MATERIAL

This supplementary material provides additional information and experiments regarding the numerical simulation and the in vivo experiments. 


\subsubsection{Numerical Simulations with Varying Simulation Parameters}

We demonstrate the effects of different parameter values on the numerical simulation. For each simulation, we keep the basic parameters as given in Table 7.2 and list all modified parameters in Table $7 \cdot 3$ of this supplementary material.

Table 7.3.: Variables and values for the numerical simulation.

\begin{tabular}{ccc}
\hline & Variable & Value \\
\hline Figure 7.7 & $\sigma_{\text {noise }}$ & 4 \\
& $C$ & 2 \\
& $D_{1}$ & 0.25 \\
& $D_{2}$ & 2.5
\end{tabular}

$\begin{array}{ccc}\text { Figure 7.8 } & \sigma_{\text {noise }} & 7 \\ & C & 3 \\ & D_{1} & 0.35 \\ & D_{2} & 3.5\end{array}$

\begin{tabular}{lll} 
Figure $7.9 \quad \Phi$ & 3 \\
\hline
\end{tabular}

For Figure 7.7, we moderately increase the noise variance and the amplitude of the spell and the trend. Consequently, the PCA performs even worse in detecting the individual oscillations, particularly for the oscillation with the higher frequency. In contrast, SSA-FARY still provides satisfying results.

For Figure 7.8, we further increase the noise variance and the amplitude of the spell and the trend. Here, the SSA-FARY result for the lower frequency oscillations are still acceptable, whereas the EOFs for the higher frequency oscillation are now also clearly corrupted.

For Figure 7.9, the same parameters for noise variance, trend and spell are used as in the main part of the manuscript, but a higher frequency variation is simulated. The PCA result is comparable to the one in the main part of the manuscript, while the SSA-FARY results look still satisfying, yet slightly worse due to the limited frequency bandwidth of the EOFs, which is determined by the window size $W$.

\subsubsection{SSA-FARY Gated bSSFP Reconstruction using Conventional Gridding}

While we have used only 30 seconds of data in the single-slice experiment described in Section 7.3 of the main part of the manuscript, we now apply SSA-FARY self-gating and PCA self-gating to the full 72 seconds of data. We use the same SSAFARY window size $W=751$ and bin the data into 25 cardiac and 3 respiratory bins. 

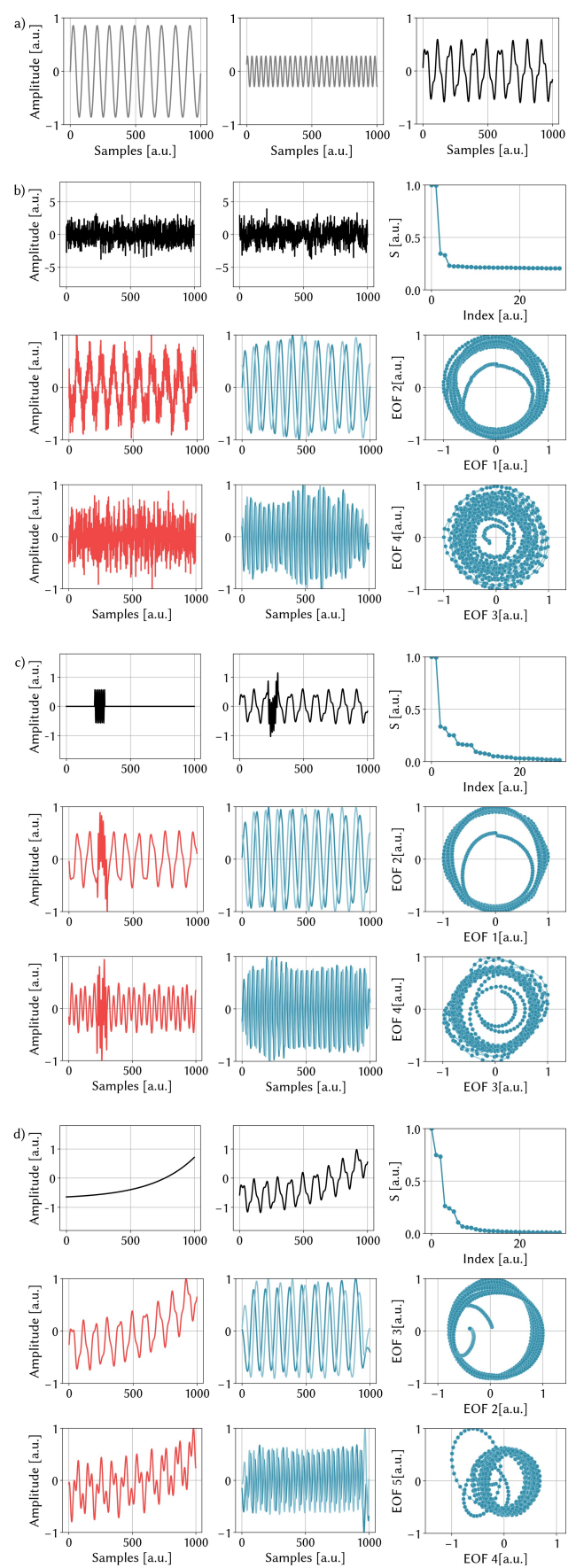

Figure 7.7:: Comparison of PCA eigenvectors and SSA-FARY EOFs. In this example there is a moderate increase of the noise variance and the spell and trend amplitude compared to Figure 7.2 of the main part of the manuscript. (a) Frequency-modulated sinusoidal oscillations $(a(t)($ left $), b(t)$ (center)) used for the numerical simulations and weighted composite signal (right). (b), (c), (d) (Black): Different additional signals (left) - (b) noise, (c) oscillatory spell, (d) exponential trend - added to the composite signal of (a) and a selected channel of the resulting signal $X_{i}(t)$ (right). (Red): First two eigenvectors of the PCA. (Blue): SSA-FARY singular values $S$ (scree plot) and EOF pairs (amplitude-time plot and amplitude-amplitude scatter plot). All plots are normalized. 

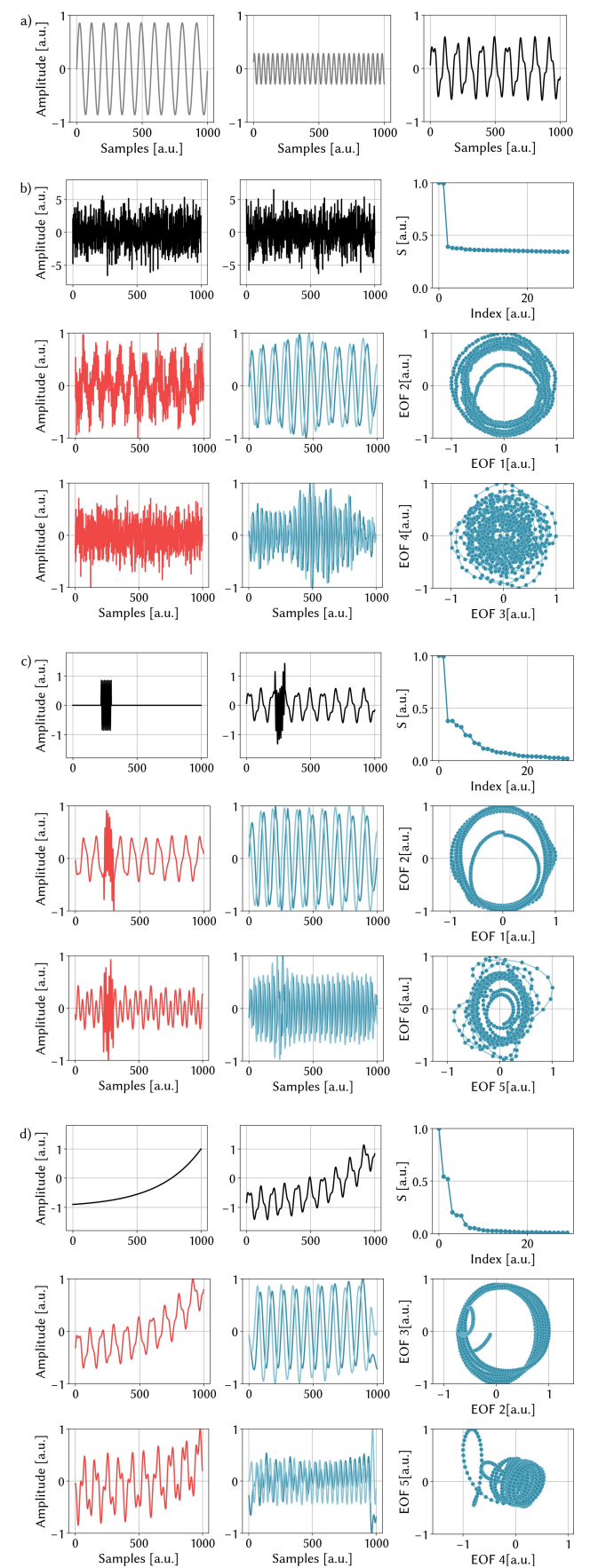

Figure 7.8.: Comparison of PCA eigenvectors and SSA-FARY EOFs. In this example there is a strong increase of the noise variance and the spell and trend amplitude compared to Figure 7.2 of the main part of the manuscript. See also caption of Figure 7.7. 

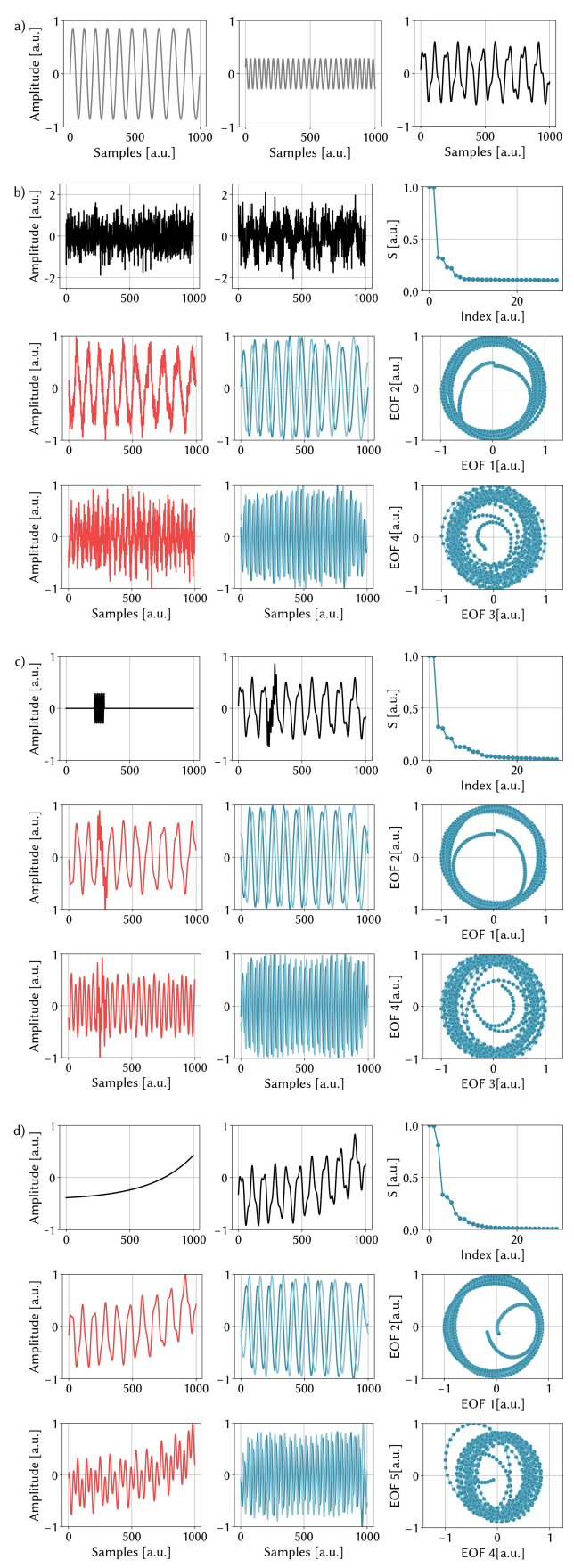

Figure 7.9.: Comparison of PCA eigenvectors and SSA-FARY EOFs. In this example there is a moderate increase of the frequency variation compared to Figure 7.2 of the main part of the manuscript. See also caption of Figure 7.7. 
On the SSA-FARY-gated data we perform a conventional gridding reconstruction followed by a Root-Sum-of-Squares (RSS) coil combination.

Similar to the results of the main part of the manuscript, SSA-FARY outperforms the PCA self-gating, which fails to separate the cardiac from respiratory motion, see Figure 7.10.

Because of the longer acquisition time and the reduced number of respiratory bins, each cardiac state contains sufficient spokes such that a conventional gridding reconstruction can be performed. While mild undersampling artifacts can be observed, the temporal dynamics are well resolved, see Figure 7.10 and movie Mov5.

Note that due to the limited number of respiratory bins we can't depict the matching respiratory state of the ECG-gated CINE breath-hold scan for the gridding reconstruction.

\subsubsection{SSA-FARY Gated Reconstructions for a Single-Slice RF-Spoiled Gradient-Echo Se- quence}

Similar to the bSSFP single-slice experiment in Section 7.3 of the main part of the manuscript, we perform a 30 second RF-spoiled gradient-echo scan (TE/TR $=1.63 / 2.60 \mathrm{~ms}$, flip angle $12^{\circ}$ ) with slice-thickness $7 \mathrm{~mm}$ of the human heart in short-axis view.

We compare the principal motion signals using PCA and SSA-FARY with window size $W=1171$, which covers a period of about $3 \mathrm{~s}$.

For each coil, Figure 7.11 depicts the DC component of 100 consecutive spokes before and after the data correction using the orthogonal projection. Just as in Figure $7 \cdot 3$ of the main part of the manuscript, some coils exhibit pronounced oscillations with approximately 15 samples per period, which is related to the seventh tiny golden angle. With the proposed correction method these oscillations can be eliminated.

Figure 7.12 shows the self-gating signals generated with PCA and SSA-FARY. In SSA-FARY, the first two EOFs represent respiratory motion and the third and fourth EOF cardiac motion. The EOFs of the pairs are in quadrature and the motion signals are well separated. PCA fails in separating the cardiac from the respiratory motion and both principle components are spoiled by noise.

Figure 7.12C shows six representative images of the SSA-FARY-gated reconstruction, three of which in end-systole and three in end-diastole. Depicted are three respiratory states from end-inspiration to end-expiration. 

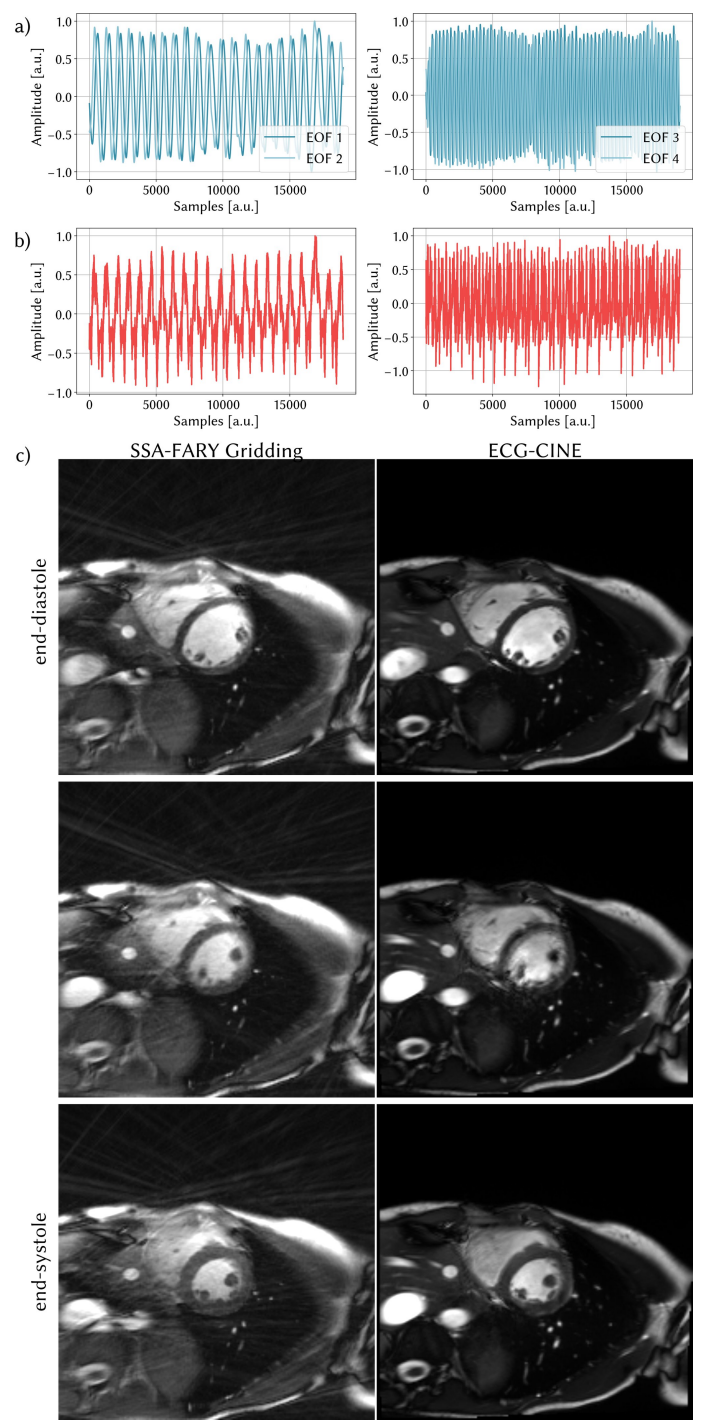

Figure 7.10.: Self-gating signals and gridding reconstruction of a single-slice radial bSSFP aquisition in comparison to conventional CINE images. In (a) the two quadrature EOFs of SSA-FARY are plotted against time for the respiratory (left) and cardiac (right) motion, respectively. In (b) the first two eigenfunctions of the PCA are depicted. For (c) the self-gating signal of (a) was utilized. It shows three representative frames of the SSA-FARY-gated gridding reconstruction corresponding to three cardiac phases from end-systole to end-diastole for a single respiration state. As in Figure 7.4 of the main manuscript, we also show vendor images of the ECG-triggered CINE breathhold scan. 
a)

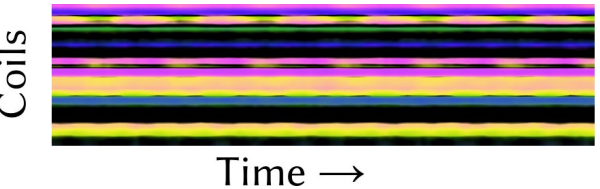

b)

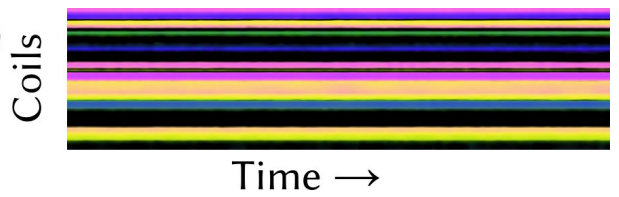

Figure 7.11.: Snippet of the amplitude plot with color-coded phase of the DC samples used for auto-calibration of the single-slice reconstruction before (a) and after (b) the data correction using the orthogonal projection. The period length of the oscillations in (a) corresponds to 15 samples.
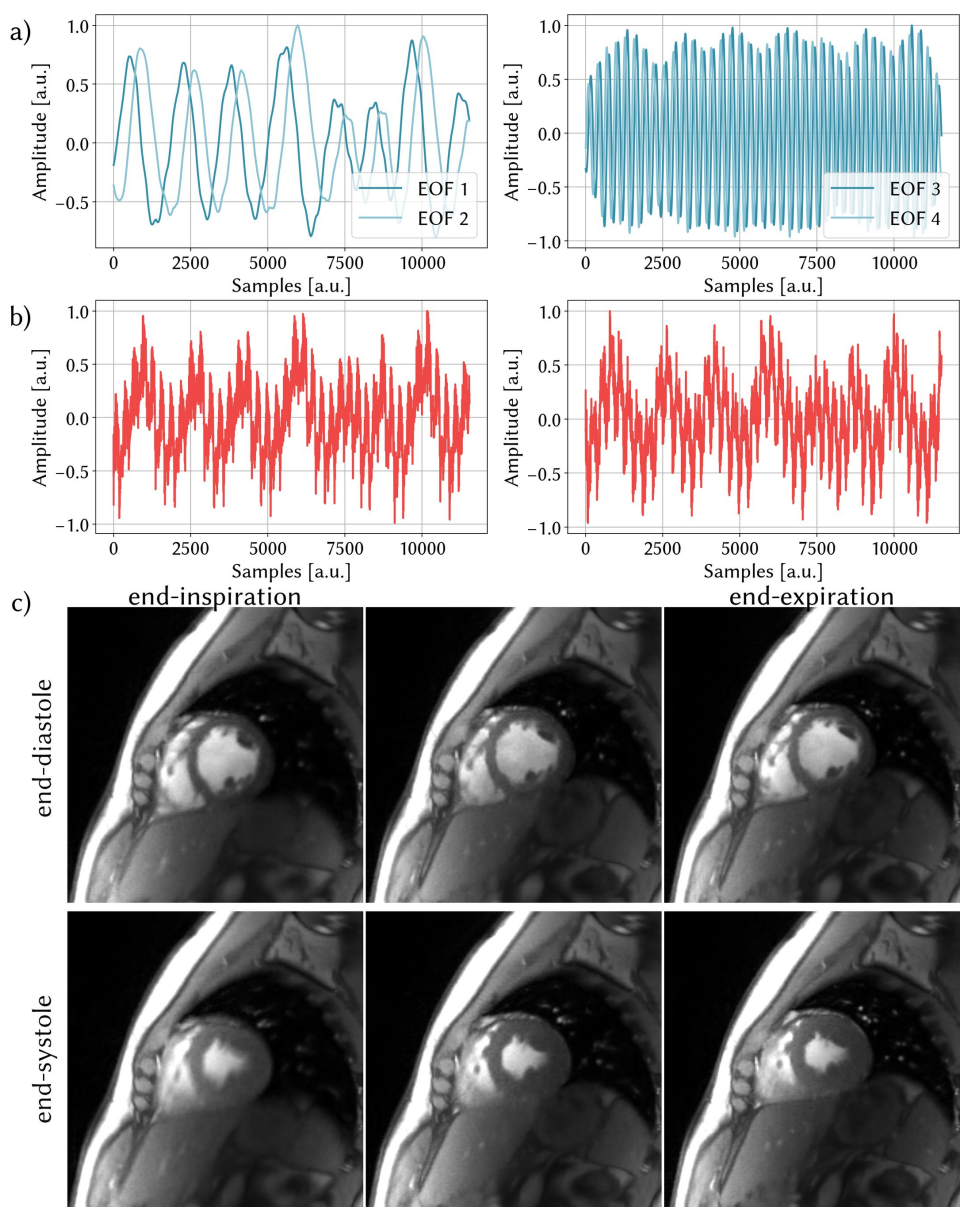

Figure 7.12.: Self-gating signals and PICS reconstruction from a single-slice radial gradient-echo acquisition. In (a) the two quadrature EOFs of SSA-FARY are plotted against time for the respiratory (left) and cardiac (right) motion, respectively. In (b) the first two eigenfunctions of the PCA are depicted. In both, a superposition of the cardiac and respiratory motion can be perceived. For (c) the self-gating signal of (a) was utilized. It shows six representative frames corresponding to end-systole and enddiastole for end-inspiration, an intermittent state and end-expiration. 


\subsubsection{Respiratory Signal Comparison for Irregular Breathing}

We perform the same SMS measurement as described in Section 7.3 of the main part of the manuscript on another volunteer who happened to have an irregular breathing pattern during the measurement. We depict the results of the experiment in Figure 7.13.

For all three slices, Figure 7.13a shows 5 out of 25 cardiac phases in end-expiration, where the individual cardiac phases can be distinguished well. From the SMSNLINV real-time reconstruction of the full time series, we determine the actual motion of the lung by extracting a line from slice one, which cuts the diaphragm in vertical direction, and use it as background for Figure 7.13b. On top, we plot a respiratory EOF obtained using SSA-FARY. It shows good agreement with the diaphragm motion in the background.

We also depict the signal from the respiratory belt, which tends to be temporarily misaligned with the diaphragm position after deep inhalation.

For the purpose of clarity, we do not plot the quadrature pair belonging to the depicted EOF. Similar to Figure 7.5 of the main part of the manuscript, the motion signals were scaled to match the amplitude of the diaphragm position displayed in the background image.

Deviations of the respiratory belt signal from the actual diaphragm position are not surprising. The correlation between external physiological motion and internal organ movement is subject dependent and patient specific signal processing can be required to account for the associated dephasing $[275,276]$, which is specifically important for irregular breathing patterns [277, 278]. Moreover, it is a know issue that the signal from a respiratory belt may be imperfect [279]. Furthermore, it was shown that an internal navigator based on the diaphragmatic motion outperforms the respiratory belt [146]. Consequently, the respiratory belt is not widely used for respiratory gating in MRI [147].

This experiment shows that - even for irregular breathing - the respiratory gating signal obtained with SSA-FARY resembles the diaphragmatic position largely well both in phase and in amplitude and therefore constitutes a suitable surrogate for respiratory gating.

7.9.5 SSA-FARY Gated Reconstructions for Highly Irregular Breathing, Yawning and a Pronounced Heart Rate Variability.

Here, we present more details on the gating and reconstruction of the SOS measurements of volunteers $\mathrm{V} 6$ and $\mathrm{V}_{7}$.

IRREgULAR BREATHING PATTERN Volunteer V6 shows a non-dictated highly irregular breathing pattern with periods of normal breathing, interrupted by vari- 
a)
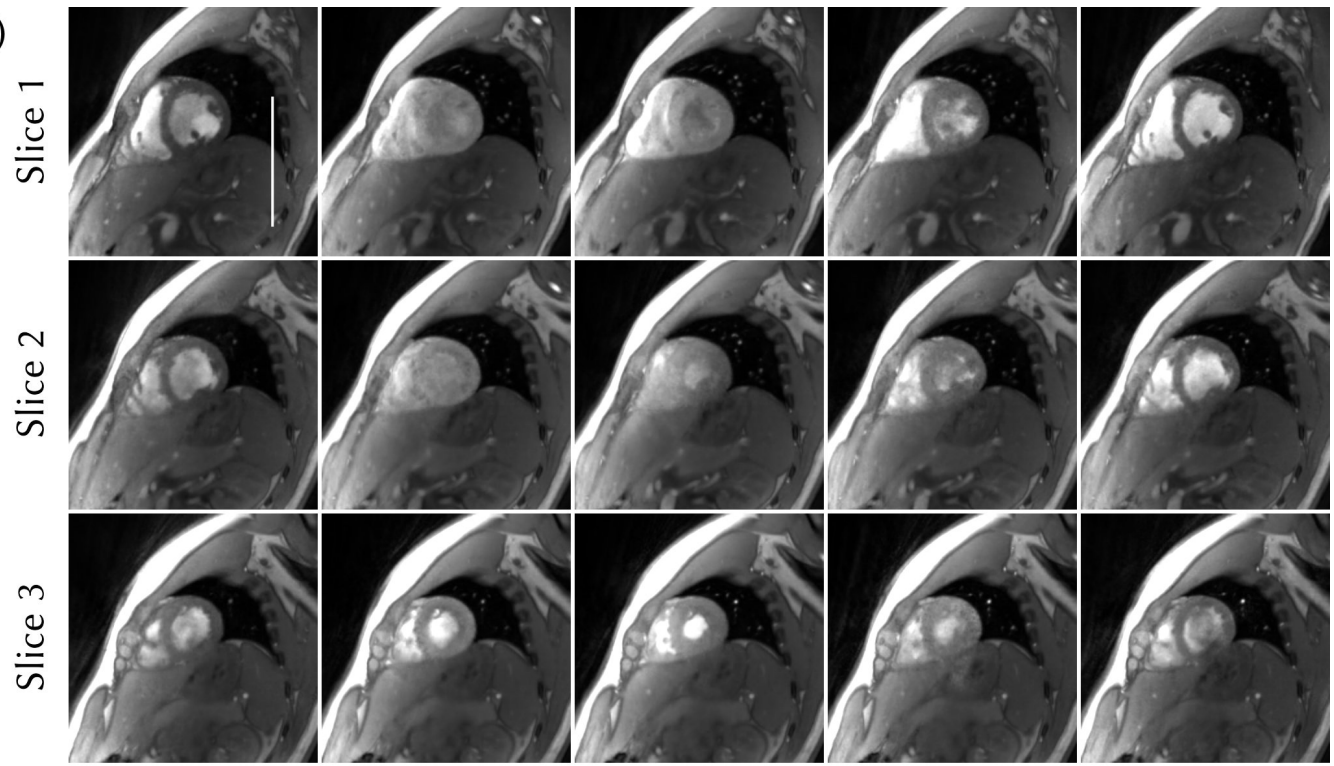

Cardiac Cycle $\rightarrow$

b)

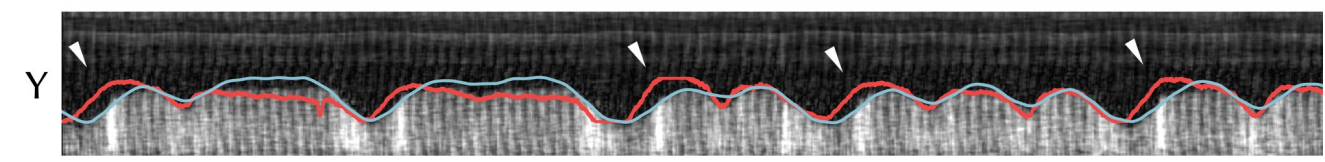

Time $\rightarrow$

Figure 7.13.: PICS reconstructions and self-gating signal of a radial SMS gradient-echo acquisition. For all three slices, (a) shows 5 out of 25 cardiac phases in end-expiration. The background of $(b)$ is extracted from a SMS-NLINV real-time reconstruction of the entire time series. It shows the temporal evolution of a vertical line (highlighted in white), placed on the diaphragm in slice one. As an overlay, the signal obtained from the respiratory belt and the EOF which is in phase with the lung motion is plotted. The signal from the respiratory belt shows some disparities compared to the real-time reconstruction after deep inhalation. This is indicated by the white arrows. 
ous breath-hold intervals of up to approximately $12 \mathrm{~s}$. The default EOF bandwidth $\delta f_{\mathrm{B}} \approx 0.35 \mathrm{~Hz}$, which here corresponds to a window size of $W=91$, is too narrow to capture this complex breathing motion in a single EOF pair. In fact, SSA-FARY creates at least $6 \mathrm{EOFs}$ to account for the respiratory motion. Consequently, the cardiac motion is only given by EOF 8 and EOF 9, see Figure 7.14a. Since for respiratory binning a single EOF pair is required, we run SSA-FARY again with reduced window size $W=51$ to increase the spectral width of the EOFs. This choice and the corresponding EOFs 2 and 3 allow for a proper binning of the respiratory motion. The window size of the moving average was adjusted to $L_{\mathrm{avg}}=600$ samples. The results for end-expiration are depicted in Figure 7.14b, together with the corresponding slices of the ECG-CINE breath-hold measurement Figure 7.14c.

YAWNING AND PRONOUNCED CARDIAC FREQUENCY VARIATIONS Volunteer $\mathrm{V}_{7}$ admitted that he unintentionally yawned during the three minute measurement. The volunteer furthermore exhibits a highly irregular heartbeat with the heart rate standard deviation of $\bar{\sigma}_{\text {heart }}=0.129 \mathrm{~Hz}$ and heart rate jumps of up to $0.3 \mathrm{~Hz}$.

Using the window size $W=91$, the three yawns are very well reflected in the first EOF, while the respiration is represented by EOFs 2 and 3, see Figure 7.15a. The first EOF was used to discard the intervals in which the volunteer yawned before binning, which reduces the for the reconstruction effectively available data about 30 s. Note that the analysis for $\sigma_{\text {trig }}$ of Table 7.1 was performed using the full data-set.

Due to the high heart rate variability, we run SSA-FARY again with window size $W=21$ to increase the spectral width of the EOFs for cardiac binning. Although such a low window-size can lead to a mixing of respiratory and cardiac oscillations, as was shown in Figure 7.6a of the main part of the manuscript, this is not the case here and the determined gating signal is in good agreement with the ECG signal, see Table 7.1. To better compensate the signal offset in the cardiac EOFs, which particularly arouse during the yawing periods, we adapted the moving average filter for the cardiac EOFs to $L_{\mathrm{avg}}=30$.

\subsubsection{Movies of the In Vivo Experiments}

For the single-slice bSSFP (Mov1-2) and RF-spoiled gradient-echo measurements (Mov3-4), we have attached two movie files, respectively. Movi and Mov3 show the cardiac cycle for end-expiration (left) and end-inspiration. Mov2 and Mov4 show the respiratory cycle for end-systole (left) and end-diastole. To account for the low number of respiratory bins, we have interpolated the frames in the respiratory resolved videos.

Mov5 shows a cardiac cycle of the gridding reconstruction. 
Mov6 shows a cardiac cycle for all three slices of the SMS reconstruction from the main part of the manuscript at end-expiration (top) and end-inspiration.

Mov7 shows a cardiac cycle for all three slices of the SMS reconstruction with erratic breathing at end-expiration.

For all analyzed volunteers $\mathrm{V}_{1}-\mathrm{V}_{7}$ of the Stack-of-Stars measurements, we have attached a representative movie (Mov8 - Mov14) showing a cardiac cycle for 6 out of 14 slices in end-expiration. 
a)
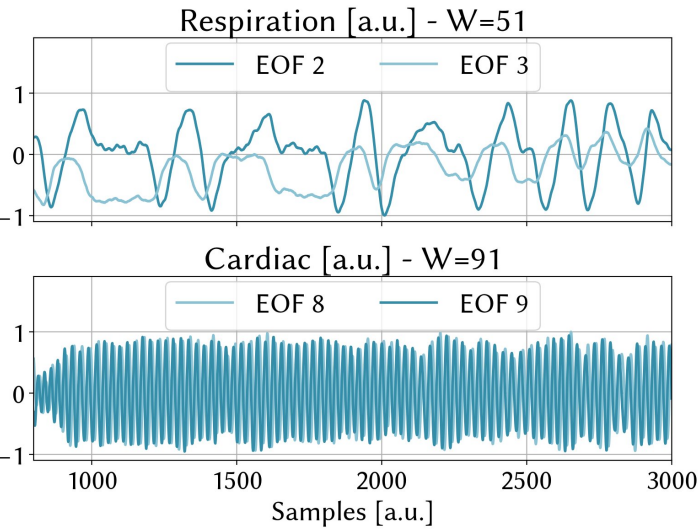

b)

SSA-FARY
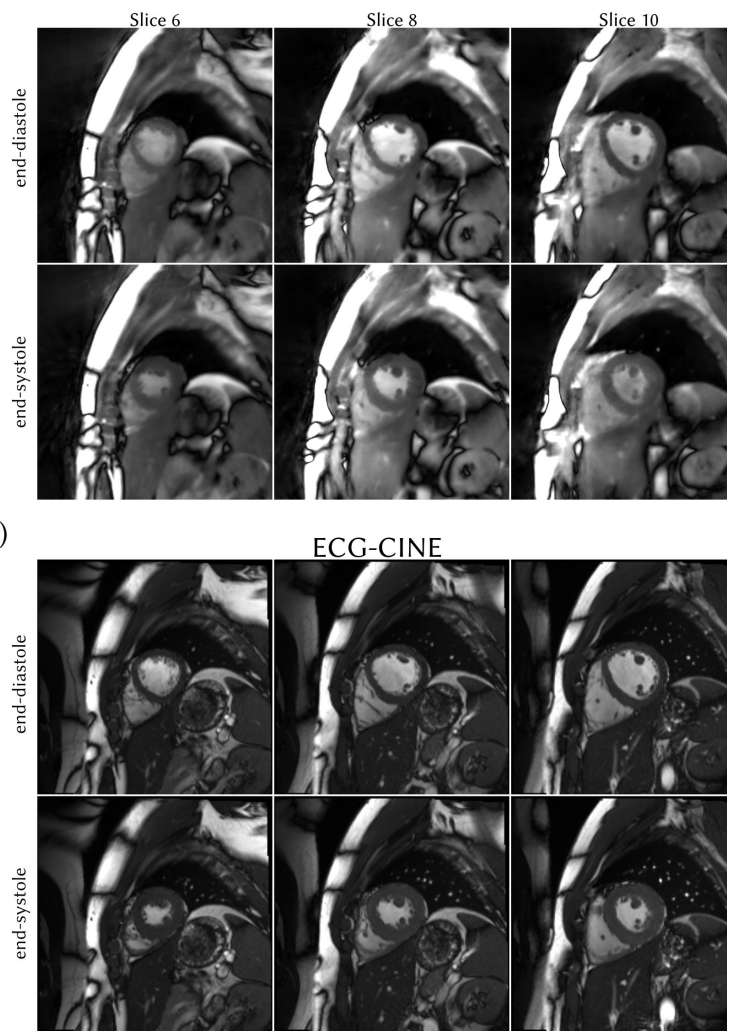

Figure 7.14.: SSA-FARY self-gating signals and representative slices from a $3 \mathrm{D}$ PICS reconstruction of a SOS bSSFP acquisition for volunteer V6 in comparison to conventional CINE images. (a) depicts a zoomed view on normalized EOF pairs representing respiratory and cardiac motion using the window sizes $W=51$ and $W=91$, respectively. (b) shows three out of fourteen slices in end-diastole and end-systole after expiration. (c) shows the corresponding reconstructions for the ECG-triggered CINE breath-hold measurement. 
a)
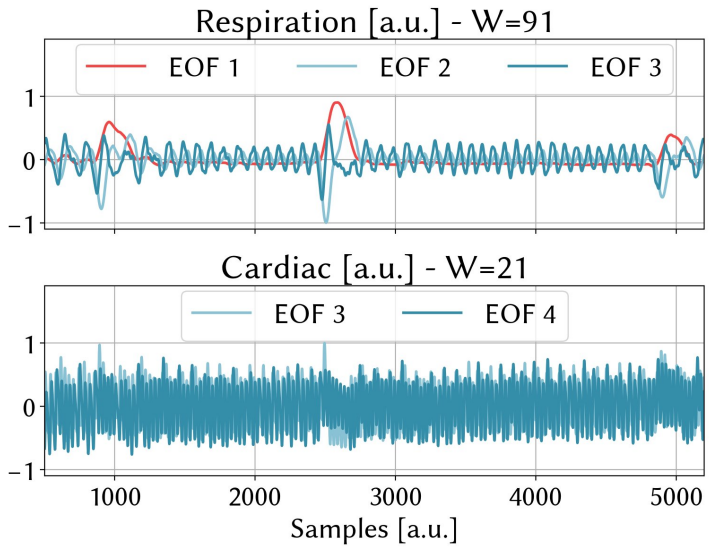

b) SSA-FARY

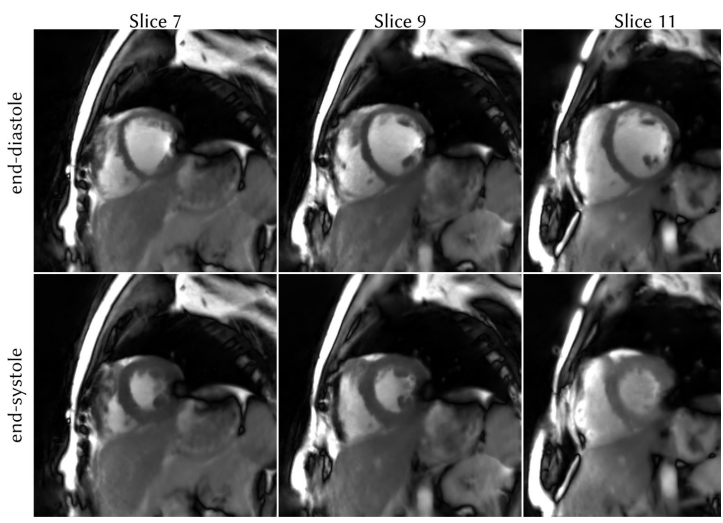

c)

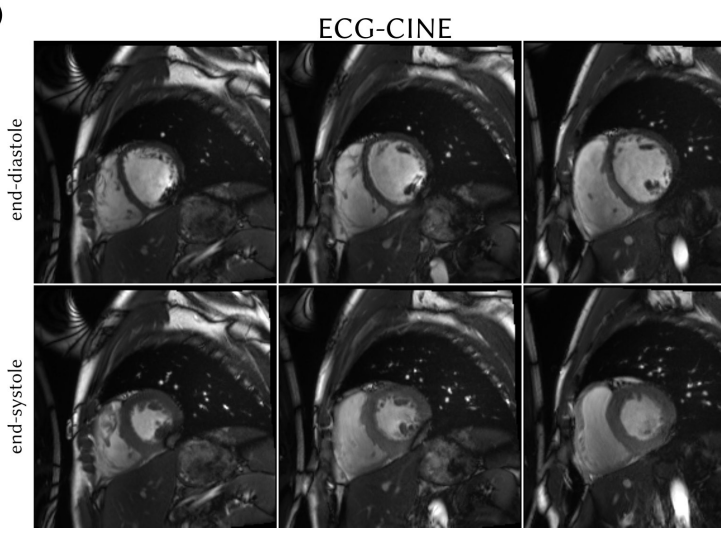

Figure 7.15:: SSA-FARY self-gating signals and representative slices from a $3 \mathrm{D}$ PICS reconstruction of a SOS bSSFP acquisition for volunteer $\mathrm{V}_{7}$ in comparison to conventional CINE images. (a) A zoomed view on the normalized EOFs 1, 2 and 3 determined with window size $W=91$. While EOF 1 captures the yawning, the EOFs 2 and 3 represent breathing motion. For window size $W=21$, the EOFs 3 and 4 capture the cardiac motion. (b) shows three out of fourteen slices in end-diastole and end-systole after expiration. (c) shows the corresponding reconstructions for the ECG-triggered CINE breath-hold measurement. 



\section{SUMMARY, OUTLOOK AND CONCLUSION}

The main goal of this thesis was the development of techniques to enable fast and robust cardiac MRI. Therefore, we have introduced three complementary methods, which bear the potential to benefit both clinically existing and emerging applications in cardiac MRI and beyond.

GRADIENT DELAY ESTIMATION WITH RING The basic prerequisite for any imaging technique is distortion-free data. Unfortunately, in MRI we often have to trade-off scan time with system imperfections, which corrupt the data acquisition. In particular, the use of fast imaging sequences, such as FLASH or bSSFP, in combination with efficient k-space sampling schemes, such as radial trajectories, lead to eddy-current-induced gradient delays, which effect inconsistent sampling. To resolve this deficiency, we have developed RING in Chapter 3. RING exploits the fact that delayed magnetic field gradients cause a miss-centering of the radial spokes, which consequently no longer intersect in the k-space center. However, the distributed intersection points can be determined and utilized to find the actual sampling locations. We could demonstrate that as few as three spokes can be enough for RING to accurately estimate gradient delays and to remove the majority of the corresponding streaking artifacts.

This capability makes RING ideally suited for interactive real-time MRI applications, where the slice position - and accordingly the gradient delays - frequently change. In Chapter 4 we showed that the RING gradient delay estimates readily adapt to positional changes of the slice, which was used to significantly reduce streaking artifacts in an interactive real-time MRI experiment.

The major limitation of the presented study is the missing online implementation to make RING accessible for on-the-fly reconstructions directly on the scanner. Despite the fact that RING does not involve computationally demanding operations, an efficient implementation and further parameter tuning will be necessary to make it compatible with the speed and latency demands of online real-time MRI.

Although introduced for conventional radial single-slice imaging, RING can also be used for SMS and SOS imaging, as it was done in Chapters 6 and 7. Recently, RING was successfully applied to flow imaging [280] and extended to compensate gradient delay errors not only for radial but also for rosette trajectories [281]. 
SimUltaneous MULTi-SLICE IMAging With SMS-NLINV Besides system imperfections, another even bigger problem is intrinsic to MRI: the slow measurement process. Hence, the only way to speed up scans is to reduce the amount of data acquired. This, however, poses high demands on the image reconstruction, which then has to yield clinically relevant results from massively undersampled data. In Chapter 5 we have therefore introduced and studied SMS-NLINV, a method which combines two established MRI techniques for fast imaging: Simultaneous Multi-Slice (SMS) and Regularized Nonlinear Inversion (NLINV). In SMS imaging, multiple slices are acquired simultaneously, which not only effects an SNR benefit but also allows for an enhanced k-space coverage, thus enabling higher undersampling factors. By the extension of the calibrationless reconstruction algorithm NLINV to SMS imaging, we have demonstrated superior image quality compared to a state-of-the-art method for highly accelerated scans.

SMS-NLINV treats image reconstruction for all slices as a joint nonlinear inverse problem, estimating both image content and coil sensitivities. As a generic method, SMS-NLINV has a large field of application. Recently, we have applied SMS-NLINV to model-based Ti mapping of multiple slices using single-shot inversion-recovery radial FLASH $[282,283]$. Furthermore, in Chapter 6 we have demonstrated the feasibility of real-time cardiac SMS imaging using SMS-NLINV. Real-time SMS-NLINV bears huge potential for future applications but requires further investigations. In particular, the implementation of an SMS bSSFP sequence would allow for time consistent dynamic imaging of multiple slices with significantly improved blood to myocardium contrast, which is fundamentally infeasible for conventional interleaved multi-slice MRI. While an efficient multi-Graphical Processor Unit (GPU) implementation of NLINV already allows for on-the-fly singleslice reconstructions [284], the joint reconstruction of multiple slices in SMS-NLINV poses even higher computational demands. Nevertheless, we are confident that further tuning of the algorithm and a suitable parallelization in combination with the steadily increasing computation power will enable real-time SMS imaging in the near future. Meanwhile, aligned SMS trajectories could be utilized, for which slicedisentangling can be performed as a pre-processing step. This will allow for an independent and thus efficient reconstruction of slices, while the time-consistency and the SNR gain provided by SMS imaging is still preserved. Real-time SMSNLINV could yield immediate improvements for various clinical studies which already rely on single-slice real-time imaging or akin techniques. Amongst others, stress testing cardiac MRI, used for cardiac parameter analysis in patients under physical exercise [285] or first-pass perfusion studies as recently demonstrated in $[286,287]$ could benefit from the SMS approach. Another considerable application is the MRI-guided targeted endomyocardial biopsy [46], where SMS real-time MRI would contribute a facilitated volumetric localization of the bioptome. 
SELF-GATING WITH SSA-FARY While multi-slice coverage is sufficient for some applications within the scope of cardiac MRI, other uses require full volumetric information about the heart, which then demands gated acquisitions. With SSAFARY we have introduced a dimensionality reduction technique for time series, which allows for robust cardiac and respiratory self-gating without the use of any external devices. At its core, SSA-FARY performs a PCA on a time-delay embedded AC region. The concept of time-delayed embedding, though simple in its construction, facilitates the detection and separation of quasi-periodic oscillations, trend and noise by involving temporal correlations in the analysis. Even though a related approach named Second-Order Blind Identification (SOBI) was recently applied to self-gated MRI as an additional signal-disentangling step [288], SSA-FARY is to the best of our knowledge - the first comprehensive and self-contained dimensionality reduction method to successfully exploit a time-delayed embedding for self-gating. It furthermore lines up with other recent approaches that employ structured low-rank matrices for image reconstruction [96, 203, 204, 289, 290], while being different in its focus on temporal signal detection and separation.

In Chapter 7, we have applied SSA-FARY to radial imaging and determined the respiratory and cardiac motion signals for single-slice, SMS and SOS freebreathing cardiac MRI experiments. The inherent data binning was successfully combined with compressed sensing image reconstruction, thereby exploiting both multi-spatial and multi-temporal correlations. In the future, we want to use this technique to generate movies of the heart with isotropic resolution. This would not only contribute a higher flexibility in the image analysis but also facilitate and speed-up the work-flow. This will be possible due to the fact that specific views of the heart can freely be chosen retrospectively by the physician and do not have to be planned explicitly in advance. First experiments imply the reliable functionality of SSA-FARY for the self-gating of isotropic data, but more effort has to be put into the acquisition side to deal with SAR limitations and artifacts arising from fat [291-293].

Besides the use for functional analysis of the heart, early trials suggest that SSAFARY can benefit a variety of other applications, which rely on gated imaging. Initial tests hint that SSA-FARY in combination with classical SSA can be used to identify sequence-imposed contrast changes as global trends, thus allowing for the detection and separation of motion signals in e.g. inversion-recovery experiments. Moreover, it gave promising results when applied to multi-echo and flow sequences. Recently, SSA-FARY was utilized to determine the motion states for an XD-NLINV reconstruction [294].

We furthermore want to stress that the capability of SSA-FARY to extract motion signals from an $\mathrm{AC}$ region offers another, entirely different approach for the imaging of dynamic processes. Instead of employing the eigenfunctions generated by SSA-FARY for cardiac and respiratory binning, they can be considered a temporal 
basis. This idea is known by the term Partially Separable Function $[173,295,296]$ and avoids the explicit determination of motion states by capturing the entire dynamics of the acquisition in a compact representation, which additionally reduces the memory demand of the reconstruction. While originally introduced employing the PCA, our prospect is that SSA-FARY can generate adequate basis functions from even smaller or noisier AC regions than current approaches.

Lately, we discovered a connection to another recently developed technique for real-time cardiac MRI by adapting the Nonlinear Laplacian Spectral Analysis (NLSA) [273], the nonlinear extension of SSA, for dimensionality reduction. The resultant method yields promising results and can be considered a delay-embedded extension of the Smoothness Regularization on Manifolds (SToRM) approach [38, 297], which uses a nonlinear manifold model to impose temporal constraints. Further inquiries are being performed to evaluate the potential of this idea.

Although exclusively applied to radial MRI in the scope of this thesis, SSA-FARY is a very general method for which it can likewise be employed for different kspace trajectories. Moreover, its domain of application as a dimensionality reduction technique for time series is not merely limited to the scope of MRI but can similarly be applied to other fields and purposes.

CONCLUSion To conclude, we have developed three novel techniques, which all contribute to the conquest of different issues, that currently hamper fast and robust cardiac MRI. RING was conceived for the elimination of gradient delay artifacts, SMS-NLINV yields efficient image reconstruction and coil sensitivity calibration and SSA-FARY allows for reliable cardio-respiratory self-gating. All these methods inherently possess broad fields of applications, but most importantly constitute essential building blocks and future prospects for fast and robust multi-dimensional cardiac MRI. 


\section{GRADIENT DELAY ESTIMATION USING THE (AC-)ADAPTIVE METHOD}

In this chapter, we will introduce the concept and a convenient implementation for the adaptive gradient delay estimation method for radial MRI developed by Block and Uecker [126, 133, 209].

Imperfect magnetic field gradient timing results in shifted k-space lines. In Chapter 3 we have shown that this shift can be modeled by

$$
\delta \boldsymbol{k}:=\boldsymbol{S} \hat{\boldsymbol{n}}_{\theta}
$$

with normalized projection direction

$$
\hat{\boldsymbol{n}}_{\theta}:=\left(\begin{array}{c}
\cos \theta \\
\sin \theta
\end{array}\right)
$$

and delay matrix

$$
S:=\left(\begin{array}{cc}
S_{x} & S_{x y} \\
S_{x y} & S_{y}
\end{array}\right)
$$

The shift along the read-out direction is therefore given by the quadratic form

$$
\delta k_{\|}=\hat{\boldsymbol{n}}^{T} \boldsymbol{S} \hat{\boldsymbol{n}} .
$$

The Adaptive method assumes that anti-parallel spokes experience a shift in diametrically opposed directions. Hence, a cross-correlation of anti-parallel spokes, which would be identical if it was not for gradient delays, should have a peak at twice the value for the actual k-space shift $\delta k_{\|}(\theta)$. To determine the delay matrix $S$ the Adaptive method starts with the acquisition of anti-parallel calibration spokes $\boldsymbol{y}_{\theta}, \boldsymbol{y}_{\theta+\pi}$ for various projection directions $\theta$. Next, a cross-correlation of both spokes is performed by application of the Fourier convolution theorem. Therefore, we flip ${ }^{f}$ one spoke, Fourier transform $\mathcal{F}$ both spokes, and multiply the complex conjugate * of the flipped and transformed spoke with its counterpart,

$$
g(r)=\mathcal{F} \boldsymbol{y}_{\theta} \cdot\left(\mathcal{F} \boldsymbol{y}_{\theta+\pi}^{f}\right)^{*}
$$

The result $g(r)$ is the Fourier transform of the cross-correlation function. A shift in one domain corresponds to a linear phase in the Fourier domain. Hence, the slope of the phase of $g(r)$ corresponds to twice the k-space shift. This concept is 
visualized in Figure A.1. The properly normalized k-space shift can therefore be obtained by

$$
\delta k_{\|}(\theta)=\frac{1}{2} \arg (\langle g(r), g(r+1)\rangle) \frac{N_{\mathrm{RO}}}{2 \pi},
$$

with $N_{\mathrm{RO}}$ the number of samples per spoke. The argument of the complex scalar product of $g(r)$ and its duplicate shifted by one pixel, $g(r+1)$, yields a magnitude weighted estimation of the slope of the phase of $g(r)$. Given the estimated shifts $\delta k_{\|}(\theta)$ for various projection angles $\theta$, the delay matrix $S$ can be determined using a least squares fit of Equation (A.6).

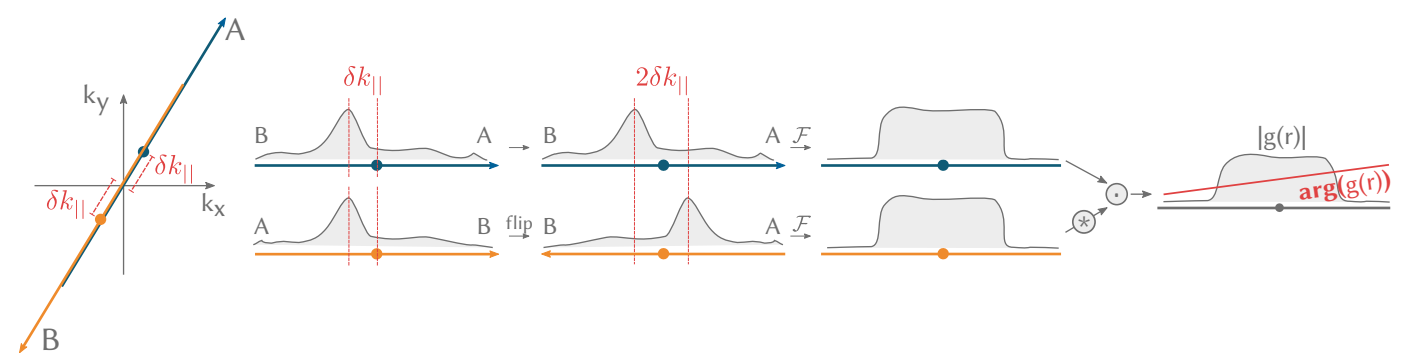

Figure A.1.: Schematic of the Adaptive method for gradient delay estimation. On the left, two anti-parallel spokes are depicted. The arrow-head denotes the read-out direction and the dot denotes the spoke's center, which without gradient delays should coincide with the k-space center. Each spoke is shifted by $\delta k_{\|}$towards its read-out direction. The letters $A$ and $B$ denote spatial positions. To the right we depict the procedure proposed by the Adaptive method. Schematically, we show the magnitude profile of the two k-space lines and highlight, that there is a mismatch between the actual center of k-space, represented by the peak in the profile, and the spoke's center. Then, one spoke gets flipped to ensure a matching spatial orientation and both spokes get Fourier transformed. Finally, the flipped and transformed spoke is complex conjugated $(*)$ and multiplied $(\cdot)$ with its counterpart. The slope of the result $g(r)$ is equivalent to twice the k-space shift $\delta k_{\|}$. The graphic was adapted from [209].

Instead of using calibration scans to acquire anti-parallel spokes, spoke pairs can also be directly picked from the actual measurement. In this so-called ACAdaptive method, (almost) anti-parallel spokes are determined form all acquired spokes to estimate the gradient delays. The AC-Adaptive method was successfully applied in various publications [44, 207, 208], although it was not always explicitly mentioned. 


\section{ACRONYMS}

\begin{tabular}{|c|c|}
\hline $\mathrm{AC}$ & auto-calibration \\
\hline ADMM & Alternating Direction Method of Multipliers \\
\hline BART & Berkeley Advanced Reconstruction Toolbox \\
\hline bSSFP & balanced Steady-State Free Precession \\
\hline CAIPIRINHA & Controlled Aliasing in Parallel Imaging Results in Higher Acceleration \\
\hline CG & Conjugate Gradient \\
\hline CT & Computed Tomography \\
\hline DC & Direct Current \\
\hline DFT & Discrete Fourier Transform \\
\hline ECG & electrocardiogram \\
\hline ENLIVE & Extended Nonlinear Inversion inspired by ESPIRiT \\
\hline $\mathrm{EOF}$ & Empirical Orthogonal Function \\
\hline EPI & Echo-Planar Imaging \\
\hline ESPIRiT & Eigenvector-based Iterative Self-Consistent Parallel Imaging Reconstruction \\
\hline FFT & Fast Fourier Transform \\
\hline FISTA & Fast Iterative Shrinkage-Thresholding Algorithm \\
\hline FLASH & Fast Low-Angle Shot \\
\hline FOV & Field of View \\
\hline GIRF & Gradient System Impulse Response Function \\
\hline GPU & Graphical Processor Unit \\
\hline IRGNM & Iteratively Regularized Gauss-Newton Method \\
\hline ISMRM & International Society for Magnetic Resonance in Medicine \\
\hline MR & Magnetic Resonance \\
\hline MRI & Magnetic Resonance Imaging \\
\hline MVUE & Minimum-Variance Unbiased Estimator \\
\hline
\end{tabular}




\begin{tabular}{|c|c|}
\hline NAV & Navigator Echoes \\
\hline NLINV & Regularized Nonlinear Inversion \\
\hline NLSA & Nonlinear Laplacian Spectral Analysis \\
\hline NMR & Nuclear Magnetic Resonance \\
\hline NUFFT & Non-Uniform Fast Fourier Transform \\
\hline PCA & Principle Component Analysis \\
\hline PICS & Parallel Imaging and Compressed Sensing \\
\hline PINS & Power Independent of Number of Slices \\
\hline $\mathrm{RF}$ & radio-frequency \\
\hline RING & Radial Spoke Intersections for Gradient Delay Estimation \\
\hline RMS & Root-Mean-Square \\
\hline ROI & Region of Interest \\
\hline RSS & Root-Sum-of-Squares \\
\hline SAKE & Structured Low-Rank Matrix Completion \\
\hline SAR & Specific Absorption Rate \\
\hline SENSE & Sensitivity Encoding \\
\hline SMS & Simultaneous Multi-Slice \\
\hline SNR & Signal-to-Noise Ratio \\
\hline SOBI & Second-Order Blind Identification \\
\hline SOS & Stack-of-Stars \\
\hline SSA & Singular Spectrum Analysis \\
\hline SSA-FARY & Singular Spectrum Analysis for Advanced Reduction of Dimensionality \\
\hline SToRM & Smoothness Regularization on Manifolds \\
\hline SVD & Singular Value Decomposition \\
\hline $\mathrm{TE}$ & Echo Time \\
\hline TR & Repetition Time \\
\hline TV & Total Variation \\
\hline VERSE & Variable-Rate Selective Excitation \\
\hline
\end{tabular}




\section{BIBLIOGRAPHY}

[1] P. C. Lauterbur. "Image Formation by Induced Local Interactions: Examples Employing Nuclear Magnetic Resonance". Nature 242 (1973), pp. 190-191 (cit. on pp. 1, 4).

[2] C. L. Partain. "The 2003 Nobel Prize for MRI: Significance and impact". J. Magn. Reson. Imaging 19 (2004), pp. 515-526 (cit. on p. 1).

[3] P. Mansfield and P. Grannell. "'Diffraction" and microscopy in solids and liquids by NMR". Phys. Rev. B 12 (1975), p. 3618 (cit. on p. 1).

[4] P. Mansfield. "Multi-planar image formation using NMR spin echoes". J. Phys. C: Solid State Phys. 10 (1977), p. L55 (cit. on p. 1).

[5] R. Damadian, M. Goldsmith, and L. Minkoff. "NMR in cancer: XVI. FONAR image of the live human body". Physiol. Chem. Phys. 9(1) (1977), pp. 97-100, 108 (cit. on p. 1).

[6] R. Hawkes, G. Holland, W. Moore, E. Roebuck, and B. Worthington. "Nuclear magnetic resonance (NMR) tomography of the normal heart". J. Comput. Assisted Tomogr. 5 (1981), pp. 605-612 (cit. on p. 1).

[7] P. A. Rinck. Magnetic Resonance in Medicine: A Critical Introduction. BoDBooks on Demand, 2019 (cit. on p. 1).

[8] F. von Knobelsdorff-Brenkenhoff, G. Pilz, and J. Schulz-Menger. "Representation of cardiovascular magnetic resonance in the AHA/ACC guidelines". J. Cardiovasc. Magn. Reson. 19 (2017), p. 70 (cit. on p. 1).

[9] F. von Knobelsdorff-Brenkenhoff and J. Schulz-Menger. "Role of cardiovascular magnetic resonance in the guidelines of the European Society of Cardiology". J. Cardiovasc. Magn. Reson. 18 (2015), p. 6 (cit. on p. 1).

[10] S. V. Babu-Narayan, G. Giannakoulas, A. M. Valente, W. Li, and M. A. Gatzoulis. "Imaging of congenital heart disease in adults". Eur. Heart J. 37 (2015), pp. 1182-1195 (cit. on p. 1).

[11] R. R. Edelman. "The History of MR Imaging as Seen through the Pages of Radiology". Radiology 273 (2014). PMID: 25340436, S181-S200 (cit. on pp. 2, 4, 20, 37).

[12] A. Haase, J. Frahm, D. Matthaei, W. Hanicke, and K.-D. Merboldt. "FLASH imaging. Rapid NMR imaging using low flip-angle pulses". J. Magn. Reson. 67 (1986), pp. 258-266 (cit. on pp. 2, 19). 
[13] D. K. Sodickson and W. J. Manning. "Simultaneous acquisition of spatial harmonics (SMASH): fast imaging with radiofrequency coil arrays". Magn. Reson. Med. 38 (1997), pp. 591-603 (cit. on pp. 2, 23).

[14] K. T. Block, M. Uecker, and J. Frahm. "Undersampled radial MRI with multiple coils. Iterative image reconstruction using a total variation constraint". Magn. Reson. Med. 57 (2007), pp. 1086-1098 (cit. on pp. 2, 25, 124).

[15] M. Lustig, D. Donoho, and J. M. Pauly. "Sparse MRI: The application of compressed sensing for rapid MR imaging". Magn. Reson. Med. 58 (2007), pp. 1182-1195 (cit. on pp. 2, 24, 25).

[16] W. Berninger, R. Redington, P. Doherty, M. Lipton, and E. Carlsson. "Gated cardiac scanning: canine studies." J. Comput. Assist. Tomo. 3 (1979), pp. 155163 (cit. on pp. 2, 35).

[17] E. Heidelberger, S. Petersen, and P. Lauterbur. "Aspects of cardiac diagnosis using synchronized NMR imaging." Eur. J. Radiol. 3 (1983), pp. 281-285 (cit. on pp. 2, 35).

[18] P. Lanzer, E. Botvinick, N. Schiller, L. Crooks, M. Arakawa, L. Kaufman, P. Davis, R. Herfkens, M. Lipton, and C. B. Higgins. "Cardiac imaging using gated magnetic resonance." Radiology 150 (1984), pp. 121-127 (cit. on pp. 2, 35).

[19] D. J. Atkinson and R. Edelman. "Cineangiography of the heart in a single breath hold with a segmented turboFLASH sequence." Radiology 178 (1991), pp. 357-360 (cit. on p. 2).

[20] V. Runge, J. Clanton, C. Partain, and A. James Jr. "Respiratory gating in magnetic resonance imaging at 0.5 Tesla." Radiology 151 (1984), pp. 521-523 (cit. on p. 2).

[21] R. L. Ehman and J. P. Felmlee. "Adaptive technique for high-definition MR imaging of moving structures." Radiology 173 (1989), pp. 255-263 (cit. on pp. 2, 38).

[22] J. Felblinger, J. Slotboom, R. Kreis, B. Jung, and C. Boesch. "Restoration of electrophysiological signals distorted by inductive effects of magnetic field gradients during MR sequences". Magn. Reson. Med. 41 (1999), pp. 715-721 (cit. on pp. 3, 35).

[23] J. W. Krug and G. Rose. "Magnetohydrodynamic distortions of the ECG in different MR scanner configurations". In: Proc. IEEE Comput. Cardiol. Vol. 38. Hangzhou, 2011, pp. 769-772 (cit. on pp. 3, 35).

[24] I. Koktzoglou, O. Simonetti, and D. Li. "Coronary artery wall imaging: initial experience at 3 Tesla". J. Magn. Reson. 21 (2005), pp. 128-132 (cit. on pp. 3, 35). 
[25] J. Suttie, L. DelaBarre, A. Pitcher, P.-F. Van de Moortele, S. Dass, C. Snyder, J. Francis, G. Metzger, P. Weale, K. Ugurbil, et al. “7 Tesla (T) human cardiovascular magnetic resonance imaging using FLASH and SSFP to assess cardiac function: validation against $1.5 \mathrm{~T}$ and 3 T." NMR Biomed. 25 (2012), pp. 27-34 (cit. on pp. 3, 37).

[26] S. Abdel-Rehim, S. Bagirathan, S. Al-Benna, and C. O'Boyle. "Burns from ECG leads in an MRI scanner: case series and discussion of mechanisms". Ann. Burn. Fire Disasters 27 (2014), p. 215 (cit. on p. 3).

[27] S. S. Vasanawala and E. Jackson. "A method of rapid robust respiratory synchronization for MRI". Pediatr. Radiol. 40 (2010), pp. 1690-1692 (cit. on p. 3).

[28] T. Geva. "Magnetic resonance imaging: historical perspective". J. Cardiovasc. Magn. Reson. 8 (2006), pp. 573-580 (cit. on p. 3).

[29] S. Zhang, A. A. Joseph, D. Voit, S. Schaetz, K.-D. Merboldt, C. UnterbergBuchwald, A. Hennemuth, J. Lotz, and J. Frahm. "Real-time magnetic resonance imaging of cardiac function and flow-Recent progress". Quant. Imaging Med. Surg. 4 (2014), pp. 313-329 (cit. on pp. 3, 53).

[30] S. G. Lingala, B. P. Sutton, M. E. Miquel, and K. S. Nayak. "Recommendations for real-time speech MRI". J. Magn. Reson. Imaging 43 (2016), pp. 28-44 (cit. on pp. 3, 53).

[31] T. S. Sorensen, D. Atkinson, T. Schaeffter, and M. S. Hansen. "Real-time reconstruction of sensitivity encoded radial magnetic resonance imaging using a graphics processing unit". IEEE Trans. Med. Imag. 28 (2009), pp. 19741985 (cit. on p. 3).

[32] R. Ordidge, P. Mansfield, M. Doyle, and R. Coupland. "Real time movie images by NMR". Brit. J. Radiol. 55 (1982), pp. 729-733 (cit. on pp. 3, 4, 17).

[33] J. Tsao, P. Boesiger, and K. P. Pruessmann. "k-t BLAST and k-t SENSE: dynamic MRI with high frame rate exploiting spatiotemporal correlations". Magn. Reson. Med. 50 (2003), pp. 1031-1042 (cit. on pp. 3, 50).

[34] M. A. Fogel, R. D. Wilson, A. Flake, M. Johnson, D. Cohen, G. McNeal, Z.-Y. Tian, and J. Rychik. "Preliminary investigations into a new method of functional assessment of the fetal heart using a novel application of 'real-time'cardiac magnetic resonance imaging". Fetal Diagn. Ther. 20 (2005), pp. $475-480$ (cit. on p. 3).

[35] F. Huang, J. Akao, S. Vijayakumar, G. R. Duensing, and M. Limkeman. "k-t GRAPPA: A k-space implementation for dynamic MRI with high reduction factor". Magn. Reson. Med. 54 (2005), pp. 1172-1184 (cit. on p. 3). 
[36] H. Jung, K. Sung, K. S. Nayak, E. Y. Kim, and J. C. Ye. "k-t FOCUSS: a general compressed sensing framework for high resolution dynamic MRI". Magn. Reson. Med. 61 (2009), pp. 103-116 (cit. on p. 3).

[37] S. G. Lingala, Y. Hu, E. DiBella, and M. Jacob. "Accelerated dynamic MRI exploiting sparsity and low-rank structure: kt SLR". IEEE Trans. Med. Imag. 30 (2011), pp. 1042-1054 (cit. on p. 3).

[38] S. Poddar and M. Jacob. "Dynamic MRI using smoothness regularization on manifolds (SToRM)". IEEE Trans. Med. Imag. 35 (2016), pp. 1106-1115 (cit. on pp. 3, 156).

[39] R. Razavi, D. L. Hill, S. F. Keevil, M. E. Miquel, V. Muthurangu, S. Hegde, K. Rhode, M. Barnett, J. van Vaals, D. J. Hawkes, et al. "Cardiac catheterisation guided by MRI in children and adults with congenital heart disease". The Lancet 362 (2003), pp. 1877-1882 (cit. on p. 3).

[40] M. Uecker, T. Hohage, K. T. Block, and J. Frahm. "Image reconstruction by regularized nonlinear inversion-joint estimation of coil sensitivities and image content". Magn. Reson. Med. 6o (2008), pp. 674-682 (cit. on pp. 3, 5, 23, 50, 66, 85, 87).

[41] M. Uecker, S. Zhang, and J. Frahm. "Nonlinear inverse reconstruction for real-time MRI of the human heart using undersampled radial FLASH". Magn. Reson. Med. 63 (2010), pp. 1456-1462 (cit. on pp. 3, 5, 23, 30, 50, 61, 66, 100, 115, 124).

[42] M. Uecker, S. Zhang, D. Voit, A. Karaus, K.-D. Merboldt, and J. Frahm. "Real-time MRI at a resolution of 20 ms". NMR Biomed. 23 (2010), pp. 986994 (cit. on pp. 3, 5, 20, 48, 50, 53, 85, 94, 100, 108, 112, 115).

[43] D. Voit, S. Zhang, C. Unterberg-Buchwald, J. M. Sohns, J. Lotz, and J. Frahm. "Real-time cardiovascular magnetic resonance at $1.5 \mathrm{~T}$ using balanced SSFP and 40 ms resolution". J. Cardiov. Magn. Reson. 15 (2013), pp. 1-8 (cit. on pp. 3, 50, 100).

[44] S. Schaetz, D. Voit, J. Frahm, and M. Uecker. "Accelerated Computing in Magnetic Resonance Imaging: Real-Time Imaging Using Nonlinear Inverse Reconstruction". Comput. Math. Method Med. 2017 (2017) (cit. on pp. 3, 62, 158).

[45] C. Unterberg-Buchwald, C. Ritter, V. Reupke, R. Wilke, M. Steinmetz, A. Schuster, J. Lotz, and M. Uecker. "P1428 Real time guidance for targeted endomyocardial biopsy in a minipig model". Eur. Heart J. 38 (2017), ehx502. P1428 (cit. on p. 3). 
[46] C. Unterberg-Buchwald, C. O. Ritter, V. Reupke, R. N. Wilke, C. Stadelmann, M. Steinmetz, A. Schuster, G. Hasenfuß, J. Lotz, and M. Uecker. "Targeted endomyocardial biopsy guided by real-time cardiovascular magnetic resonance". J. Cardiov. Magn. Reson. 19 (2017), p. 45 (cit. on pp. 3, 61, 154).

[47] V. Rasche, R. W. D. Boer, D. Holz, and R. Proksa. "Continuous radial data acquisition for dynamic MRI". Magn. Reson. Med. 34 (1995), pp. 754-761 (cit. on p. 4).

[48] G. H. Glover and J. M. Pauly. "Projection Reconstruction Techniques for Reduction of Motion Effects in MRI". Magn. Reson. Med. 28 (1992), pp. 275289 (cit. on pp. 4, 37).

[49] J. M. Pauly. "Gridding \& the NUFFT for non-Cartesian image reconstruction". In: Proc. Intl. Soc. Mag. Reson. Med. Vol. 20. Melbourne, 2012 (cit. on pp. 4, 27).

[5o] D. C. Peters, J. A. Derbyshire, and E. R. McVeigh. “Centering the projection reconstruction trajectory: Reducing gradient delay errors". Magn. Reson. Med. 50 (2003), pp. 1-6 (cit. on pp. 4, 33, 61-63, 71, 72).

[51] W. Kim, C. Mun, D. Kim, and Z. Cho. "Extraction of cardiac and respiratory motion cycles by use of projection data and its applications to NMR imaging". Magn. Reson. Med. 13 (1990), pp. 25-37 (cit. on pp. 4, 53, 115).

[52] A. C. Larson, R. D. White, G. Laub, E. R. McVeigh, D. Li, and O. P. Simonetti. "Self-gated cardiac cine MRI". Magn. Reson. Med. 51 (2004), pp. 93-102 (cit. on pp. 4, 53, 115, 117, 135).

[53] M. E. Crowe, A. C. Larson, Q. Zhang, J. Carr, R. D. White, D. Li, and O. P. Simonetti. "Automated rectilinear self-gated cardiac cine imaging". Magn. Reson. Med. 52 (2004), pp. 782-788 (cit. on pp. 4, 115).

[54] M. Buehrer, J. Curcic, P. Boesiger, and S. Kozerke. "Prospective self-gating for simultaneous compensation of cardiac and respiratory motion". Magn. Reson. Med. 6o (2008), pp. 683-69o (cit. on pp. 4, 115).

[55] S. Uribe, V. Muthurangu, R. Boubertakh, T. Schaeffter, R. Razavi, D. L. G. Hill, and M. S. Hansen. "Whole-heart cine MRI using real-time respiratory self-gating". Magn. Reson. Med. 57 (2007), pp. 606-613 (cit. on pp. 4, 53, 115).

[56] J. Pang, B. Sharif, Z. Fan, X. Bi, R. Arsanjani, D. S. Berman, and D. Li. “ECG and navigator-free four-dimensional whole-heart coronary MRA for simultaneous visualization of cardiac anatomy and function". Magn. Reson. Med. 72 (2014), pp. 1208-1217 (cit. on pp. 4, 55, 115, 118). 
[57] J. Paul, E. Divkovic, S. Wundrak, P. Bernhardt, W. Rottbauer, H. Neumann, and V. Rasche. "High-resolution respiratory self-gated golden angle cardiac MRI: comparison of self-gating methods in combination with k-t SPARSE SENSE". Magn. Reson. Med. 73 (2015), pp. 292-298 (cit. on pp. 4, 53, 115, 121).

[58] S. Rosenzweig, H. C. M. Holme, and M. Uecker. "Simple auto-calibrated gradient delay estimation from few spokes using Radial Intersections (RING)". Magn. Reson. Med. 81 (2019), pp. 1898-1906 (cit. on pp. 4, 59, 110, 123, 190).

[59] S. Rosenzweig, H. Holme, and M. Uecker. "Simple Auto-Calibrated Gradient Delay Estimation From Few Spokes Using Radial Intersections (RING) for Interactive Real-time MRI". In: Proc. Intl. Soc. Mag. Reson. Med. Vol. 27. Montreal, 2019, p. 445 (cit. on pp. 4, 79, 123, 190).

[6o] S. Rosenzweig, H. C. M. Holme, R. N. Wilke, D. Voit, J. Frahm, and M. Uecker. "Simultaneous multi-slice MRI using cartesian and radial FLASH and regularized nonlinear inversion: SMS-NLINV". Magn. Reson. Med. 79 (2018), pp. 2057-2066 (cit. on pp. 5, 71, 83, 123, 124, 190).

[61] S. Rosenzweig, H. C. M. Holme, N. Scholand, R. N. Wilke, and M. Uecker. "Self-Gated and Real-time Simultaneous Multi-Slice Cardiac MRI from the Same Acquisition". In: Proc. Intl. Soc. Mag. Reson. Med. Vol. 26. Paris, 2018, p. 0209 (cit. on pp. 5, 53, 107, 115, 191).

[62] S. Rosenzweig, H. C. M. Holme, R. N. Wilke, and M. Uecker. "Simultaneous Multi-Slice Real-Time Imaging with Radial Multi-Band FLASH and Nonlinear Inverse Reconstruction". In: Proc. Intl. Soc. Mag. Reson. Med. Vol. 24. Honolulu, 2017, p. 0518 (cit. on pp. 5, 71, 99, 100, 107, 124, 191).

[63] S. Rosenzweig. "Simultaneous multi-slice reconstruction by Regularized Nonlinear Inversion". Master's Thesis. Georg August Universität Göttingen, 2016 (cit. on pp. 5, 7, 14, 40, 41, 83, 112).

[64] S. Rosenzweig, N. Scholand, H. Holme, and M. Uecker. "Robust Cardiac and Respiratory Self-Gating Using an Adapted Singular Spectrum Analysis (SSA-FARI): Application to Simultaneous-Multi-Slice Imaging". In: Proc. Intl. Soc. Mag. Reson. Med. Vol. 27. Montreal, 2019, p. 2444 (cit. on pp. 5, 116, 191).

[65] S. Rosenzweig, N. Scholand, H. Holme, and M. Uecker. "Robust Cardiac and Respiratory Self-Gating using an adapted Singular Spectrum Analysis". In: Proc. Soc. Cardiovasc. Magn. Reson. Vol. 22. Bellevue, 2019, pp. 580-582 (cit. on pp. $5,116,191)$. 
[66] R. W. Brown, Y.-C. N. Cheng, E. M. Haacke, M. R. Thompson, and R. Venkatesan. Magnetic resonance imaging: physical principles and sequence design. John Wiley \& Sons, 2014 (cit. on p. 7).

[67] Z. P. Liang and P. C. Lauterbur. Principles of Magnetic Resonance Imaging: A Signal Processing Perspective. IEEE Press Series on Biomedical Engineering. Wiley, 1999 (cit. on pp. 7, 23).

[68] M. H. Levitt. Spin dynamics: basics of nuclear magnetic resonance. John Wiley \& Sons, 2001 (cit. on pp. 7, 9).

[69] R. H. Hashemi, W. G. Bradley, and C. J. Lisanti. MRI: the basics. Lippincott Williams \& Wilkins, 2012 (cit. on p. 7).

[70] R. M. Dreizler and C. S. Lüdde. Theoretische Physik 3: Quantenmechanik 1. Springer-Lehrbuch. Springer Berlin Heidelberg, 2007 (cit. on pp. 7, 8).

[71] F. Schwabl. Quantenmechanik (QM I): Eine Einführung. Springer-Lehrbuch. Springer Berlin Heidelberg, 2013 (cit. on p. 7).

[72] W. Pauli. "Über den Zusammenhang des Abschlusses der Elektronengruppen im Atom mit der Komplexstruktur der Spektren". Zs. Phys. A Hadr. Nucl. 31 (1925), pp. 765-783 (cit. on p. 7).

[73] A. Karaus. "Aufbau und Anwendung von Verfahren der Magnetresonanztomografie mit stimulierten Echos". PhD thesis. Bayrische Julius-MaximiliansUniversität Würzburg, 2010 (cit. on p. 7).

[74] B. M. Dale, M. A. Brown, and R. C. Semelka. MRI: Basic Principles and Applications. Wiley, 2015 (cit. on p. 8).

[75] C. Cohen-Tannoudji, B. Diu, and F. Laloë. Quantenmechanik. Quantenmechanik. de Gruyter, 2007 (cit. on p. 8).

[76] L. G. Hanson. "Is quantum mechanics necessary for understanding magnetic resonance?" Concepts Magn. Reso. A 32A (2008), pp. 329-340 (cit. on pp. 8, 9).

[77] G. Findenegg and T. Hellweg. Statistische Thermodynamik. Springer Berlin Heidelberg, 2015 (cit. on p. 9).

[78] F. Bloch. "Nuclear Induction". Phys. Rev. 70 (1946), pp. 460-474 (cit. on p. 10).

[79] P. Sprawls. Magnetic resonance imaging: principles, methods, and techniques. Medical Physics Publishing, 2000 (cit. on p. 11).

[8o] M. Bryon. MRI transmitter, receiver, and coils. uRL: http: //www . mouser . de/ applications/medical-imaging-overview/ (visited on 10/30/2019) (cit. on p. 12). 
[81] M. A. Bernstein, K. F. King, and X. J. Zhou. Handbook of MRI Pulse Sequences. Elsevier Science, 2004 (cit. on pp. 13, 14, 16, 31-33, 43).

[82] D. A. Yablonskiy, A. L. Sukstanskii, and J. J. Ackerman. "Image artifacts in very low magnetic field MRI: the role of concomitant gradients". J. Magn. Reson. 174 (2005), pp. 279-286 (cit. on p. 13).

[83] S. Narayanan, K. Nayak, S. Lee, A. Sethy, and D. Byrd. “An approach to realtime magnetic resonance imaging for speech production". J. Acoust. Soc. Am. 115 (2004), pp. 1771-1776 (cit. on p. 17).

[84] K. T. Block and J. Frahm. "Spiral imaging: a critical appraisal". J. Magn. Reson. Imaging 21 (2005), pp. 657-668 (cit. on p. 18).

[85] I. C. Atkinson, A. Lu, and K. R. Thulborn. "Characterization and correction of system delays and eddy currents for MR imaging with ultrashort echotime and time-varying gradients". Magn. Reson. Med. 62 (2009), pp. 532-537 (cit. on pp. 18, 33).

[86] D. G. Kruger, G. S. Slavin, R. Muthupillai, R. C. Grimm, and S. J. Riederer. "An orthogonal correlation algorithm for ghost reduction in MRI". Magn. Reson. Med. 38 (1997), pp. 678-686 (cit. on p. 18).

[87] M. Katoh, E. Spuentrup, A. Buecker, W. J. Manning, R. W. Günther, and R. M. Botnar. "MR coronary vessel wall imaging: Comparison between radial and spiral k-space sampling". J. Magn. Reson. Imaging 23 (2006), pp. 757762 (cit. on p. 18).

[88] K. T. Block. "Advanced methods for radial data sampling in magnetic resonance imaging". PhD thesis. Georg August Universität Göttingen, 2008 (cit. on pp. $18,28,47$ ).

[89] B. Hargreaves. "Rapid gradient-echo imaging". J. Magn. Reson. Imaging 36 (2012), pp. 1300-1313 (cit. on p. 20).

[9o] V. Roeloffs, D. Voit, and J. Frahm. "Spoiling without additional gradients: Radial FLASH MRI with randomized radiofrequency phases". Magn. Reson. Med. 75 (2016), pp. 2094-2099 (cit. on pp. 20, 79, 123).

[91] A. Oppelt, R. Graumann, H. Barfuss, H. Fischer, W. Hartl, and W. Schajor. "FISP-a new fast MRI sequence". Electromedica 54 (1986), pp. 15-18 (cit. on p. 20).

[92] H. Thiele, E. Nagel, I. Paetsch, B. Schnackenburg, A. Bornstedt, M. Kouwenhoven, A. Wahl, G. Schuler, and E. Fleck. "Functional cardiac MR imaging with steady-state free precession (SSFP) significantly improves endocardial border delineation without contrast agents". J. Magn. Reson. Imaging 14 (2001), pp. 362-367 (cit. on p. 20). 
[93] M. A. Griswold, P. M. Jakob, R. M. Heidemann, M. Nittka, V. Jellus, J. Wang, B. Kiefer, and A. Haase. "Generalized autocalibrating partially parallel acquisitions (GRAPPA)". Magn. Reson. Med. 47 (2002), pp. 1202-1210 (cit. on pp. 21, 23, 85).

[94] K. P. Pruessmann, M. Weiger, M. B. Scheidegger, and P. Boesiger. "SENSE: sensitivity encoding for fast MRI". Magn. Reson. Med. 42 (1999), pp. 952-962 (cit. on pp. 21, 23, 85, 93).

[95] P. B. Roemer, W. A. Edelstein, C. E. Hayes, S. P. Souza, and O. M. Mueller. "The NMR phased array". Magn. Reson. Med. 16 (1990), pp. 192-225 (cit. on p. 22).

[96] M. Uecker, P. Lai, M. J. Murphy, P. Virtue, M. Elad, J. M. Pauly, S. S. Vasanawala, and M. Lustig. "ESPIRiT—an eigenvalue approach to autocalibrating parallel MRI: where SENSE meets GRAPPA". Magn. Reson. Med. 71 (2014), pp. 990-1001 (cit. on pp. 22, 85, 93, 99, 118, 124, 155).

[97] H. C. M. Holme, S. Rosenzweig, F. Ong, R. N. Wilke, M. Lustig, and M. Uecker. "ENLIVE: An Efficient Nonlinear Method for Calibrationless and Robust Parallel Imaging". Sci. Rep. 9 (2019), p. 3034 (cit. on pp. 23, 190).

[98] M. Uecker. “Nonlinear Reconstruction Methods for Parallel Magnetic Resonance Imaging". PhD thesis. Georg-August-Universität Göttingen, 2009 (cit. on p. 23).

[99] E. H. Moore. "On the reciprocal of the general algebraic matrix". Bull. Am. Math. Soc. 26 (1920), pp. 394-395 (cit. on p. 24).

[10o] R. Penrose. "A generalized inverse for matrices". In: Math. Proc. Camb. Philos. Soc. Vol. 51. Cambridge University Press. 1955, pp. 406-413 (cit. on p. 24).

[101] M. James. "The Generalised Inverse". Math. Gaz. 62 (1978), pp. 109-114 (cit. on p. 24).

[102] A. N. Tikhonov, A. Goncharsky, V. Stepanov, and A. G. Yagola. Numerical methods for the solution of ill-posed problems. Vol. 328. Springer Science \& Business Media, 2013 (cit. on p. 24).

[103] S. Boyd and L. Vandenberghe. Convex Optimization. Cambridge University Press, 2004 (cit. on pp. 24-26).

[104] M. W. Marcellin, M. J. Gormish, A. Bilgin, and M. P. Boliek. “An overview of JPEG-200o". In: Proc. Data Comp. Conf. Vol. 10. Snowbird, 2000, pp. 523541 (cit. on p. 25).

[105] D. L. Donoho. "Compressed sensing". IEEE Trans. Inform. Theory 52 (2006), pp. 1289-1306 (cit. on p. 25). 
[106] A. Beck and M. Teboulle. "A Fast Iterative Shrinkage-Thresholding Algorithm for Linear Inverse Problems". SIAM J. Img. Sci. 2 (2009), pp. 183-202 (cit. on p. 25).

[107] A. Chambolle and T. Pock. "A First-Order Primal-Dual Algorithm for Convex Problems with Applications to Imaging". J. Math. Imaging Vis. 40 (2011), pp. 120-145 (cit. on p. 25).

[108] S. Boyd, N. Parikh, E. Chu, B. Peleato, and J. Eckstein. "Distributed Optimization and Statistical Learning via the Alternating Direction Method of Multipliers". Found. Trends Mach. Learn. 3 (2011), pp. 1-122 (cit. on pp. 2527, 124).

[109] M. R. Hestenes and E. Stiefel. "Methods of conjugate gradients for solving linear systems". J. Res. Nat. Bur. Stand. 49 (1952) (cit. on p. 25).

[110] K. P. Pruessmann, M. Weiger, P. Boernert, and P. Boesiger. "Advances in sensitivity encoding with arbitrary k-space trajectories". Magn. Reson. Med. 46 (2001), pp. 638-651 (cit. on p. 26).

[111] M. Hanke. Conjugate gradient type methods for ill-posed problems. CRC Press, 2017 (cit. on p. 26).

[112] R. Glowinski and A. Marroco. "Sur l'approximation, par éléments finis d'ordre un, et la résolution, par pénalisation-dualité d'une classe de problèmes de Dirichlet non linéaires". ESAIM 9 (1975), pp. 41-76 (cit. on p. 26).

[113] D. Gabay and B. Mercier. "A dual algorithm for the solution of nonlinear variational problems via finite element approximation". Comput. Math. Appl. 2 (1976), pp. 17-40 (cit. on p. 26).

[114] N. Parikh and S. Boyd. "Proximal algorithms". Found. Trends Optim. 1 (2014), pp. 123-231 (cit. on p. 27).

[115] A. Beck and M. Teboulle. "Fast Gradient-Based Algorithms for Constrained Total Variation Image Denoising and Deblurring Problems". IEEE Trans. Image Processing 18 (2009), pp. 2419-2434 (cit. on p. 27).

[116] J. W. Cooley and J. W. Tukey. "An Algorithm for the Machine Calculation of Complex Fourier Series". Math. Comput. 19 (1965), pp. 297-301 (cit. on p. 27).

[117] J. D. O'Sullivan. "A fast sinc function gridding algorithm for Fourier inversion in computer tomography". IEEE Trans. Med. Imag. 4 (1985), pp. 200-207 (cit. on pp. 27, 28).

[118] P. J. Beatty, D. G. Nishimura, and J. M. Pauly. "Rapid gridding reconstruction with a minimal oversampling ratio". IEEE Trans. Med. Imag. 24 (2005), pp. 799-8o8 (cit. on p. 27). 
[119] J. Pauly. Non-cartesian reconstruction. 2007. URL: http : / / mri-q . com / uploads /3/4/5/7/34572113/pauly-non-cartesian_recon.pdf (visited on $02 / 01 / 2020$ ) (cit. on pp. 27, 29).

[120] J. I. Jackson, C. H. Meyer, D. G. Nishimura, and A. Macovski. "Selection of a convolution function for Fourier inversion using gridding". IEEE Trans. Med. Imag. 10 (1991), pp. $473-478$ (cit. on p. 28).

[121] F. T. A. W. Wajer and K. P. Pruessmann. "Major speedup of reconstruction for sensitivity encoding with arbitrary trajectories". In: Proc. Intl. Soc. Mag. Reson. Med. Vol. 9. Glasgow, 2001, p. 0767 (cit. on pp. 30, 88).

[122] F. Aurenhammer. "Voronoi diagrams-a survey of a fundamental geometric data structure". ACM Comput. Surv. 23 (1991), pp. 345-405 (cit. on p. 30).

[123] P. M. Joseph. "Sampling errors in projection reconstruction MRI". Magn. Reson. Med. 40 (1998), pp. 460-466 (cit. on p. 30).

[124] G. Ramachandran and A. Lakshminarayanan. "Three-dimensional reconstruction from radiographs and electron micrographs: application of convolutions instead of Fourier transforms". PNAS 68 (1971), pp. 2236-2240 (cit. on p. 30).

[125] T. B. Smith and K. S. Nayak. "MRI artifacts and correction strategies". Imaging Med. 2 (2010), pp. 445-457 (cit. on p. 31).

[126] A. Moussavi, M. Untenberger, M. Uecker, and J. Frahm. “Correction of gradient-induced phase errors in radial MRI". Magn. Reson. Med. 71 (2013), pp. 308-312 (cit. on pp. 31, 32, 62, 63, 71, 81, 157).

[127] P. Jehenson, M. Westphal, and N. Schuff. "Analytical method for the compensation of eddy-current effects induced by pulsed magnetic field gradients in NMR systems". J. Magn. Reson. 90 (1990), pp. 264-278 (cit. on pp. 32, 33).

[128] J. Van Vaals and A. Bergman. "Optimization of eddy-current compensation". J. Magn. Reson. 90 (1990), pp. 52-70 (cit. on p. 32).

[129] C. Ahn and Z. Cho. "A new phase correction method in NMR imaging based on autocorrelation and histogram analysis". IEEE Trans. Med. Imaging 6 (1987), pp. 32-36 (cit. on p. 33).

[130] H. M. Gach, I. J. Lowe, D. P. Madio, A. Caprihan, S. A. Altobelli, D. O. Kuethe, and E. Fukushima. "A programmable pre-emphasis system". Magn. Reson. Med. 40 (1998), pp. 427-431 (cit. on p. 33).

[131] R. Wysong, D. Madio, and I. J. Lowe. "A novel eddy current compensation scheme for pulsed gradient systems". Magn. Reson. Med. 31 (1994), pp. 572575 (cit. on p. 33). 
[132] S. Crozier, C. D. Eccles, F. A. Beckey, J. Field, and D. M. Doddrell. "Correction of eddy-current-induced Bo shifts by receiver reference-phase modulation". J. Magn. Reson. 97 (1992), pp. 661-665 (cit. on p. 33).

[133] K. T. Block and M. Uecker. "Simple Method for Adaptive Gradient-Delay Compensation in Radial MRI". In: Proc. Intl. Soc. Mag. Reson. Med. Vol. 19. Montreal, 2011, p. 2816 (cit. on pp. 34, 59, 62, 69, 157).

[134] M. Untenberger, Z. Tan, D. Voit, A. A. Joseph, V. Roeloffs, K. D. Merboldt, S. Schätz, and J. Frahm. "Advances in real-time phase-contrast flow MRI using asymmetric radial gradient echoes". Magn. Reson. Med. 75 (2016), pp. 19011908 (cit. on pp. 34, 59, 62, 69).

[135] A. N. Shetty. "Suppression of radiofrequency interference in cardiac gated MRI: a simple design". Magn. Reson. Med. 8 (1988), pp. 84-88 (cit. on p. 35).

[136] S. Skare and J. L. Andersson. "On the effects of gating in diffusion imaging of the brain using single shot EPI". Magn. Reson. Imaging 19 (2001), pp. 11251128 (cit. on p. 37).

[137] B. Fletcher, M. Jacobstein, A. Nelson, T. Riemenschneider, and R. Alfidi. "Gated magnetic resonance imaging of congenital cardiac malformations." Radiology 150 (1984), pp. 137-140 (cit. on p. 37).

[138] J. Oster and G. D. Clifford. "Acquisition of electrocardiogram signals during magnetic resonance imaging". Physiol. Meas. 38 (2017), Ri19 (cit. on p. 37).

[139] I. Burger and E. M. Meintjes. "Elliptical subject-specific model of respiratory motion for cardiac MRI". Magn. Reson. Med. 70 (2013), pp. 722-731 (cit. on pp. 37, 121).

[140] M. Usman, G. Vaillant, D. Atkinson, T. Schaeffter, and C. Prieto. "Compressive manifold learning: Estimating one-dimensional respiratory motion directly from undersampled k-space data". Magn. Reson. Med. 72 (2014), pp. 1130-1140 (cit. on p. 37).

[141] R. Xu, P. Athavale, P. Krahn, K. Anderson, J. Barry, L. Biswas, V. Ramanan, N. Yak, M. Pop, and G. A. Wright. "Respiratory motion model based correction for improving the targeting accuracy of MRI-guided intracardiac electrophysiology procedures". J. Cardiov. Magn. Reson. 17 (2015), pp. 1-3 (cit. on p. 37).

[142] C. Prieto, M. Doneva, M. Usman, M. Henningsson, G. Greil, T. Schaeffter, and R. M. Botnar. "Highly efficient respiratory motion compensated freebreathing coronary mra using golden-step Cartesian acquisition". J. Magn. Reson. Imaging 41 (2015), pp. 738-746 (cit. on p. 37). 
[143] L. Feng, L. Axel, H. Chandarana, K. T. Block, D. K. Sodickson, and R. Otazo. "XD-GRASP: Golden-angle radial MRI with reconstruction of extra motionstate dimensions using compressed sensing". Magn. Reson. Med. 75 (2016), pp. 775-788 (cit. on pp. 37, 43, 51, 55, 115, 117, 121, 124, 135, 137).

[144] R. L. Ehman, M. McNamara, M. Pallack, H. Hricak, and C. Higgins. "Magnetic resonance imaging with respiratory gating: techniques and advantages". Am. J. Roentgenol. 143 (1984), pp. 1175-1182 (cit. on pp. 37, 115).

[145] C. Lewis, F. Prato, D. Drost, and R. Nicholson. "Comparison of respiratory triggering and gating techniques for the removal of respiratory artifacts in MR imaging." Radiology 160 (1986), pp. 803-810 (cit. on p. 37).

[146] G. Li, J. Wei, D. Olek, M. Kadbi, N. Tyagi, K. Zakian, J. Mechalakos, J. O. Deasy, and M. Hunt. "Direct comparison of respiration-correlated fourdimensional magnetic resonance imaging reconstructed using concurrent internal navigator and external bellows". Int. J. Radiat. Oncol. Biol. Phys. 97 (2017), pp. 596-605 (cit. on pp. 37, 146).

[147] J. R. McClelland, D. J. Hawkes, T. Schaeffter, and A. P. King. "Respiratory motion models: a review". Med. Image Anal. 17 (2013), pp. 19-42 (cit. on pp. 37, 146).

[148] D. J. Larkman, J. V. Hajnal, A. H. Herlihy, G. A. Coutts, I. R. Young, and G. Ehnholm. "Use of multicoil arrays for separation of signal from multiple slices simultaneously excited". J. Magn. Reson. Imaging 13 (2001), pp. 313317 (cit. on pp. 39, 85).

[149] S. Moeller, E. Yacoub, C. A. Olman, E. Auerbach, J. Strupp, N. Harel, and K. Ugurbil. "Multiband multislice GE-EPI at 7 tesla, with 16-fold acceleration using partial parallel imaging with application to high spatial and temporal whole-brain fMRI". Magn. Reson. Med. 63 (2010), pp. 1144-1153 (cit. on pp. 39, 85).

[150] D. A. Feinberg, S. Moeller, S. M. Smith, E. Auerbach, S. Ramanna, M. F. Glasser, K. L. Miller, K. Ugurbil, and E. Yacoub. "Multiplexed echo planar imaging for sub-second whole brain FMRI and fast diffusion imaging". PLoS One 5 (2010), e15710 (cit. on pp. 39, 85).

[151] S. P. Souza, J. Szumowski, C. L. Dumoulin, D. P. Plewes, and G. Glover. "SIMA: simultaneous multislice acquisition of MR images by Hadamardencoded excitation." J. Comput. Assist. Tomogr. 12 (1988), pp. 1026-1030 (cit. on pp. 39, 85).

[152] F. A. Breuer, M. Blaimer, R. M. Heidemann, M. F. Mueller, M. A. Griswold, and P. M. Jakob. "Controlled aliasing in parallel imaging results in higher acceleration (CAIPIRINHA) for multi-slice imaging". Magn. Reson. Med. 53 (2005), pp. 684-691 (cit. on pp. 40, 85, 90, 93). 
[153] S. R. Yutzy, N. Seiberlich, J. L. Duerk, and M. A. Griswold. "Improvements in multislice parallel imaging using radial CAIPIRINHA". Magn. Reson. Med. 65 (2011), pp. 1630-1637 (cit. on pp. 40, 85, 90).

[154] M. Barth, F. Breuer, P. J. Koopmans, D. G. Norris, and B. A. Poser. "Simultaneous multislice (SMS) imaging techniques". Magn. Reson. Med. 75 (2016), pp. $63-81$ (cit. on p. 41).

[155] J. Hennig. "Chemical shift imaging with phase-encoding RF pulses". Magn. Reson. Med. 25 (1992), pp. 289-298 (cit. on p. 41).

[156] G. Goelman. "Two methods for peak RF power minimization of multiple inversion-band pulses". Magn. Reson. Med. 37 (1997), pp. 658-665 (cit. on p. 41).

[157] E. J. Auerbach, J. Xu, E. Yacoub, S. Moeller, and K. Uğurbil. "Multiband accelerated spin-echo echo planar imaging with reduced peak RF power using time-shifted RF pulses". Magn. Reson. Med. 69 (2013), pp. 1261-1267 (cit. on p. 41).

[158] D. Stäb, C. O. Ritter, F. A. Breuer, A. M. Weng, D. Hahn, and H. Köstler. "CAIPIRINHA accelerated SSFP imaging". Magn. Reson. Med. 65 (2011), pp. 157-164 (cit. on pp. 42, 100, 112).

[159] J. Duerk, M. Griswold, and K. Dara. Multi-slice blipped TrueFISPCAIPIRINHA. US Patent 9,086,468. 2015 (cit. on pp. 42, 43, 112).

[160] S. T. Wong and M. S. Roos. "A strategy for sampling on a sphere applied to 3 D selective RF pulse design". Magn. Reson. Med. 32 (1994), pp. 778-784 (cit. on p. 43).

[161] D. Piccini, A. Littmann, S. Nielles-Vallespin, and M. O. Zenge. "Spiral phyllotaxis: the natural way to construct a $3 \mathrm{D}$ radial trajectory in MRI". Magn. Reson. Med. 66 (2011), pp. 1049-1056 (cit. on p. 43).

[162] K. T. Block, H. Chandarana, S. Milla, M. Bruno, T. Mulholland, G. Fatterpekar, M. Hagiwara, R. Grimm, C. Geppert, and B. Kiefer. "Towards routine clinical use of radial stack-of-stars $3 \mathrm{~d}$ gradient-echo sequences for reducing motion sensitivity". J. Korean Soc. Magn. Reson. Med. 18 (2014), pp. 87-106 (cit. on pp. 43, 61, 62, 71).

[163] Y.-F. Yen, K. F. Han, B. L. Daniel, S. Heiss, R. L. Birdwell, R. J. Herfkens, A. M. Sawyer-Glover, and G. H. Glover. "Dynamic breast MRI with spiral trajectories: 3D versus 2D". J. Magn. Reson. Imaging 11 (2000), pp. 351-359 (cit. on p. 43). 
[164] L. Feng, T. Zhao, H. Chandarana, D. K. Sodickson, and R. Otazo. "MotionResolved Golden-Angle Radial Sparse MRI Using Variable-Density Stackof-Stars Sampling". In: Proc. Intl. Soc. Mag. Reson. Med. Vol. 24. Singapore, 2016, p. 1091 (cit. on pp. 43, 48, 123).

[165] T. Wech, W. Pickl, J. Tran-Gia, C. Ritter, M. Beer, D. Hahn, and H. Köstler. "Whole-heart cine MRI in a single breath-hold-a compressed sensing accelerated $3 \mathrm{D}$ acquisition technique for assessment of cardiac function". Rö. Fo. 186 (2014), pp. 37-41 (cit. on p. 43).

[166] Z. Zhou, F. Han, L. Yan, D. J. Wang, and P. Hu. “Golden-ratio rotated stackof-stars acquisition for improved volumetric MRI". Magn. Reson. Med. 78 (2017), pp. 2290-2298 (cit. on pp. 43, 71, 90, 123).

[167] S. Winkelmann, T. Schaeffter, T. Koehler, H. Eggers, and O. Doessel. “An optimal radial profile order based on the Golden Ratio for time-resolved MRI". IEEE Trans. Med. Imag. 26 (2007), pp. 68-76 (cit. on p. 48).

[168] O. Bieri and K. Scheffler. "Flow compensation in balanced SSFP sequences". Magn. Reson. Med. 54 (2005), pp. 901-907 (cit. on pp. 48, 134).

[169] S. Wundrak, J. Paul, J. Ulrici, E. Hell, M.-A. Geibel, P. Bernhardt, W. Rottbauer, and V. Rasche. "Golden ratio sparse MRI using tiny golden angles". Magn. Reson. Med. 75 (2016), pp. 2372-2378 (cit. on p. 48).

[170] S. Wundrak, J. Paul, J. Ulrici, E. Hell, and V. Rasche. "A small surrogate for the golden angle in time-resolved radial MRI based on generalized fibonacci sequences". IEEE Trans. Med. Imag. 34 (2015), pp. 1262-1269 (cit. on pp. 48, $62,123,134)$.

[171] F. A. Breuer, P. Kellman, M. A. Griswold, and P. M. Jakob. "Dynamic autocalibrated parallel imaging using temporal GRAPPA (TGRAPPA)". Magn. Reson. Med. 53 (2005), pp. 981-985 (cit. on p. 50).

[172] P. Kellman, F. H. Epstein, and E. R. McVeigh. "Adaptive sensitivity encoding incorporating temporal filtering (TSENSE)". Magn. Reson. Med. 45 (2001), pp. 846-852 (cit. on p. 50).

[173] Z.-P. Liang. "Spatiotemporal imaging with partially separable functions". In: IEEE Int. Symp. Biomed. Imag. Vol. 4. Arlington, 2007, pp. 988-991 (cit. on pp. 50, 156).

[174] C. Bilen, I. W. Selesnick, Y. Wang, R. Otazo, D. Kim, L. Axel, and D. K. Sodickson. "On compressed sensing in parallel MRI of cardiac perfusion using temporal wavelet and TV regularization". In: IEEE Int. Conf. Acoust. Speech Sig. Proc. Vol. 35. Dallas, 2010, pp. 630-633 (cit. on p. 50). 
[175] A. G. Christodoulou, J. L. Shaw, C. Nguyen, Q. Yang, Y. Xie, N. Wang, and D. Li. "Magnetic resonance multitasking for motion-resolved quantitative cardiovascular imaging". Nat. Biomed. Eng. 2 (2018), p. 215 (cit. on pp. 53, 137).

[176] A. Majumdar. "FOCUSS based Schatten-p norm minimization for real-time reconstruction of dynamic contrast enhanced MRI". IEEE Signal Process. Lett. 19 (2012), pp. 315-318 (cit. on p. 53).

[177] X. Zhang et al. "3D self-gated cardiac cine imaging at 3 Tesla using stack-ofstars bSSFP with tiny golden angles and compressed sensing". Magn. Reson. Med. 81 (2019), pp. 3234-3244 (cit. on pp. 53, 134).

[178] N. H. Packard, J. P. Crutchfield, J. D. Farmer, and R. S. Shaw. "Geometry from a time series". Phys. Rev. Lett. 45 (1980), p. 712 (cit. on p. 55).

[179] F. Takens. "Detecting strange attractors in turbulence". In: Dynamical Systems and Turbulence (Warwick 1980). Lecture Notes in Mathematics. Vol. 898. Berlin, Heidelberg: Springer, Berlin, Heidelberg, 1981, pp. 366-381 (cit. on pp. 55, 116, 120).

[18o] H. Kantz and T. Schreiber. Nonlinear Time Series Analysis. 2nd ed. Cambridge University Press, 2003 (cit. on pp. 55-57).

[181] E. Bradley and H. Kantz. "Nonlinear time-series analysis revisited". Chaos 25 (2015), p. 097610 (cit. on pp. 55, 57).

[182] P. Castiglione, M. Falcioni, A. Lesne, and A. Vulpiani. Chaos and coarse graining in statistical mechanics. Cambridge University Press Cambridge, 2008 (cit. on p. 56).

[183] O. E. Rössler. "An equation for continuous chaos". Phys. Lett. A 57 (1976), pp. 397-398 (cit. on p. 56).

[184] T. Sauer, J. A. Yorke, and M. Casdagli. “Embedology". J. Stat. Phys. 65 (1991), pp. 579-616 (cit. on p. 56).

[185] M. Casdagli, S. Eubank, J. D. Farmer, and J. Gibson. "State space reconstruction in the presence of noise". Physica D 51 (1991), pp. 52-98 (cit. on p. 57).

[186] D. S. Broomhead and G. P. King. "Extracting qualitative dynamics from experimental data". Physica D 20 (1986), pp. 217-236 (cit. on pp. 58, 116).

[187] H. K. Song and L. Dougherty. "k-Space weighted image contrast (KWIC) for contrast manipulation in projection reconstruction MRI". Magn. Reson. Med. 44 (2000), pp. 825-832 (cit. on p. 61).

[188] C. A. Mistretta, O. Wieben, J. Velikina, W. Block, J. Perry, K. Wu Y.Johnson, and Y. Wu. "Highly constrained backprojection for time-resolved MRI". Magn. Reson. Med. 55 (2006), pp. 30-40 (cit. on p. 61). 
[189] K. L. Wright, J. I. Hamilton, M. A. Griswold, V. Gulani, and N. Seiberlich. "Non-Cartesian parallel imaging reconstruction". J. Magn. Reson. Imaging 40 (2014), pp. 1022-1040 (cit. on p. 61).

[190] C. Barmet, N. D. Zanche, and K. P. Pruessmann. "Spatiotemporal magnetic field monitoring for MR". Magn. Reson. Med. 6o (2008), pp. 187-197 (cit. on pp. 61, 134).

[191] B. E. Dietrich, D. O. Brunner, B. J. Wilm, C. Barmet, S. Gross, L. Kasper, M. Haeberlin, T. Schmid, S. J. Vannesjo, and K. P. Pruessmann. "A field camera for MR sequence monitoring and system analysis". Magn. Reson. Med. 75 (2016), pp. 1831-1840 (cit. on p. 61).

[192] S. J. Vannesjo, M. Haeberlin, L. Kasper, M. Pavan, B. J. Wilm, C. Barmet, and K. P. Pruessmann. "Gradient system characterization by impulse response measurements with a dynamic field camera". Magn. Reson. Med. 69 (2013), pp. 583-593 (cit. on pp. 61, 134).

[193] H. Liu and G. B. Matson. "Accurate measurement of magnetic resonance imaging gradient characteristics". Materials 7 (2014), pp. 1-15 (cit. on p. 61).

[194] H. Jang and A. B. McMillan. "A rapid and robust gradient measurement technique using dynamic single-point imaging". Magn. Reson. Med. 78 (2017), pp. 950-962 (cit. on p. 61).

[195] M. Stich, T. Wech, A. Slawig, R. Ringler, A. Dewdney, A. Greiser, G. Ruyters, T. A. Bley, and H. Köstler. "Gradient waveform pre-emphasis based on the gradient system transfer function". Magn. Reson. Med. 80 (2018), pp. 15211532 (cit. on pp. 61, 134).

[196] A. Deshmane, M. Blaimer, F. Breuer, P. Jakob, J. Duerk, N. Seiberlich, and M. Griswold. "Self-calibrated trajectory estimation and signal correction method for robust radial imaging using GRAPPA operator gridding". Magn. Reson. Med. 75 (2016), pp. 883-896 (cit. on pp. 61, 134).

[197] T. Wech, J. Tran-Gia, T. A. Bley, and H. Köstler. “Using self-consistency for an iterative trajectory adjustment (SCITA)". Magn. Reson. Med. 73 (2015), pp. 1151-1157 (cit. on p. 61).

[198] N. Seiberlich, F. A. Breuer, M. Blaimer, K. Barkauskas, P. M. Jakob, and M. A. Griswold. "Non-Cartesian data reconstruction using GRAPPA operator gridding (GROG)". Magn. Reson. Med. 58 (2007), pp. 1257-1265 (cit. on pp. 61, 134).

[199] A. B. Kerr, J. M. Pauly, B. S. Hu, K. C. Li, C. J. Hardy, C. H. Meyer, A. Macovski, and D. G. Nishimura. "Real-time interactive MRI on a conventional scanner". Magn. Reson. Med. 38 (1997), pp. 355-367 (cit. on pp. 61, 115). 
[200] M. A. Guttman, K. Peter, D. A. J., L. R. J., and M. E. R. “Real-time accelerated interactive MRI with adaptive TSENSE and UNFOLD". Magn. Reson. Med. 50 (2003), pp. 315-321 (cit. on p. 61).

[201] P. C. Yang, A. B. Kerr, A. C. Liu, D. H. Liang, C. Hardy, C. H. Meyer, A. Macovski, J. M. Pauly, and B. S. Hu. "New real-time interactive cardiac magnetic resonance imaging system complements echocardiography". J. Am. Coll. Cardiol. 32 (1998), pp. 2049-2056 (cit. on pp. 61, 115).

[202] W. Jiang, P. E. Larson, and M. Lustig. "Simultaneous auto-calibration and gradient delays estimation (SAGE) in non-Cartesian parallel MRI using lowrank constraints". Magn. Reson. Med. 8o (2018), pp. 2006-2016 (cit. on p. 61).

[203] P. J. Shin, P. E. Z. Larson, M. A. Ohliger, M. Elad, J. M. Pauly, D. B. Vigneron, and M. Lustig. "Calibrationless parallel imaging reconstruction based on structured low-rank matrix completion". Magn. Reson. Med. 72 (2014), pp. 959-970 (cit. on pp. 61, 155).

[204] M. Mani, V. Magnotta, and M. Jacob. "A general algorithm for compensation of trajectory errors: Application to radial imaging". Magn. Reson. Med. 8o (2018), pp. 1605-1613 (cit. on pp. 61, 155).

[205] J. D. Ianni and W. A. Grissom. "Trajectory Auto-Corrected image reconstruction". Magn. Reson. Med. 76 (2016), pp. 757-768 (cit. on p. 61).

[206] L. Feng, R. Grimm, K. T. Block, H. Chandarana, S. Kim, J. Xu, L. Axel, D. K. Sodickson, and R. Otazo. "Golden-angle radial sparse parallel MRI: Combination of compressed sensing, parallel imaging, and golden-angle radial sampling for fast and flexible dynamic volumetric MRI". Magn. Reson. Med. 72 (2014), pp. 707-717 (cit. on p. 62).

[207] X. Wang, V. Roeloffs, J. Klosowski, Z. Tan, D. Voit, M. Uecker, and J. Frahm. "Model-based T1 mapping with sparsity constraints using single-shot inversion-recovery radial FLASH". Magn. Reson. Med. 79 (2018), pp. 730740 (cit. on pp. 62, 158).

[208] V. Roeloffs, X. Wang, T. J. Sumpf, M. Untenberger, D. Voit, and J. Frahm. "Model-based reconstruction for T1 mapping using single-shot inversionrecovery radial FLASH". Int. J. Imag. Syst. Tech. 26 (2016), pp. 254-263 (cit. on pp. 62, 158).

[209] S. Rosenzweig, H. C. M. Holme, R. N. Wilke, and M. Uecker. "Extending the Simple Method for Adaptive Gradient-Delay Compensation in Radial MRI". In: Proc. Intl. Soc. Mag. Reson. Med. Vol. 26. Paris, 2018, p. 4207 (cit. on pp. 62, 69, 70, 157, 158, 191).

[210] M. Uecker, F. Ong, J. I. Tamir, D. Bahri, P. Virtue, J. Y. Cheng, T. Zhang, and M. Lustig. "Berkeley advanced reconstruction toolbox". In: Proc. Intl. Soc. Mag. Reson. Med. Vol. 23. Toronto, 2015, p. 2486 (cit. on pp. 65, 93, 124). 
[211] H. Haji-Valizadeh et al. "Validation of highly accelerated real-time cardiac cine MRI with radial k-space sampling and compressed sensing in patients at $1.5 \mathrm{~T}$ and $3 \mathrm{~T}^{\prime \prime}$. Magn. Reson. Med. 79 (2018), pp. 2745-2751 (cit. on pp. 66, $70)$.

[212] H. C. M. Holme, S. Rosenzweig, F. Ong, R. N. Wilke, M. Lustig, and M. Uecker. "ENLIVE: An Efficient Nonlinear Method for Calibrationless and Robust Parallel Imaging". arXiv (2017) (cit. on p. 66).

[213] H. Wang, G. Adluru, L. Chen, E. G. Kholmovski, N. K. Bangerter, and E. V. R. DiBella. "Radial simultaneous multi-slice CAIPI for ungated myocardial perfusion". Magn. Reson. Imaging 34 (2016), pp. 1329-1336 (cit. on pp. 71, 85).

[214] M. Lustig and J. M. Pauly. "SPIRiT: Iterative self-consistent parallel imaging reconstruction from arbitrary k-space". Magn. Reson. Med. 64 (2010), pp. 457-471 (cit. on p. 85).

[215] A. A. Maudsley. "Multiple-line-scanning spin density imaging". J. Magn. Reson. 41 (1980), pp. 112-126 (cit. on p. 85).

[216] M. Weiger, K. P. Pruessmann, and P. Boesiger. "2D sense for faster 3 D MRI". Magn. Reson. Mater. Phys., Biol. Med. 14 (2002), pp. 10-19 (cit. on pp. 85, 99).

[217] F. Wiesinger, P. Boesiger, and K. P. Pruessmann. "Electrodynamics and ultimate SNR in parallel MR imaging". Magn. Reson. Med. 52 (2004), pp. 376390 (cit. on p. 85).

[218] K. Setsompop, B. A. Gagoski, J. R. Polimeni, T. Witzel, V. J. Wedeen, and L. L. Wald. "Blipped-controlled aliasing in parallel imaging for simultaneous multislice echo planar imaging with reduced g-factor penalty". Magn. Reson. Med. 67 (2012), pp. 1210-1224 (cit. on p. 85).

[219] S. Rosenzweig and M. Uecker. "Reconstruction of multiband MRI data using Regularized Nonlinear Inversion". In: Proc. Intl. Soc. Mag. Reson. Med. Vol. 24. Singapore, 2016, p. 3259 (cit. on pp. 87, 192).

[220] F. Knoll, C. Clason, M. Uecker, and R. Stollberger. "Improved Reconstruction in Non-Cartesian Parallel Imaging by Regularized Nonlinear Inversion". In: Proc. Intl. Soc. Mag. Reson. Med. Vol. 17. Honolulu, 2009, p. 2721 (cit. on p. 88).

[221] K. Zhu, A. Kerr, and J. M. Pauly. "Autocalibrating CAIPIRINHA: Reformulating CAIPIRINHA as a 3 D Problem". In: Proc. Intl. Soc. Mag. Reson. Med. Vol. 20. Melbourne, 2012, p. 0518 (cit. on pp. 90, 93). 
[222] S. Zhang, M. Uecker, D. Voit, K. Merboldt, and J. Frahm. "Real-time cardiovascular magnetic resonance at high temporal resolution: radial FLASH with nonlinear inverse reconstruction". J. Cardiov. Magn. Reson. 12 (2010), p. 39 (cit. on p. 100).

[223] Z. Wu, W. Chen, M. C. K. Khoo, S. L. Davidson Ward, and K. S. Nayak. "Evaluation of upper airway collapsibility using real-time MRI". J. Magn. Reson. Imaging 44 (2016), pp. 158-167 (cit. on p. 100).

[224] M. Uecker, K. T. Block, and J. Frahm. "Nonlinear Inversion with L1-Wavelet Regularization - Application to Autocalibrated Parallel Imaging". In: Proc. Intl. Soc. Mag. Reson. Med. Vol. 16. Toronto, 2008, p. 1479 (cit. on p. 100).

[225] F. Knoll, K. Bredies, T. Pock, and R. Stollberger. "Second order total generalized variation (TGV) for MRI". Magn. Reson. Med. 65 (2011), pp. 480-491 (cit. on p. 100).

[226] J. Bundy, O. Simonetti, G. Laub, and J. Finn. "Segmented TrueFISP cine imaging of the heart". In: Proc. Intl. Soc. Magn. Reson. Med. Vol. 7. Philadelphia, 1999, p. 1282 (cit. on p. 112).

[227] S. Rosenzweig, N. Scholand, H. C. M. Holme, and M. Uecker. "Cardiac and Respiratory Self-Gating in Radial MRI using an Adapted Singular Spectrum Analysis (SSA-FARY)". IEEE Trans. Med. Imag. (2020). doi: 10.1109/TMI.2020.2985994 (cit. on pp. 113, 190).

[228] Y. L. Liu, S. J. Riederer, P. J. Rossman, R. C. Grim, J. P. Debbins, and R. L. Ehman. "A monitoring, feedback, and triggering system for reproducible breath-hold MR imaging". Magn. Reson. Med. 30 (1993), pp. 507-511 (cit. on p. 115).

[229] L. Guo. "Novel Techniques in Projection-based Motion Tracking in Cardiac Magnetic Resonance Imaging". PhD thesis. Johns Hopkins University, 2017 (cit. on p. 115).

[23o] C. B. Higgins and H. Hricak. Magnetic resonance imaging of the body. Elsevier, 1987 (cit. on p. 115).

[231] R. Rokey, R. E. Wendt, and D. L. Johnston. "Monitoring of acutely III patients during nuclear magnetic resonance imaging: Use of a Time-varying filter electrocardiographic gating device to reduce gradient artifacts". Magn. Reson. Med. 6 (1988), pp. 240-245 (cit. on p. 115).

[232] A. C. Larson, P. Kellman, A. Arai, G. A. Hirsch, E. McVeigh, D. Li, and O. P. Simonetti. "Preliminary investigation of respiratory self-gating for freebreathing segmented cine MRI". Magn. Reson. Med. 53 (2005), pp. 159-168 (cit. on p. 115). 
[233] M. Kirby. Geometric Data Analysis: An Empirical Approach to Dimensionality Reduction and the Study of Patterns. John Wiley \& Sons, Inc., 2000 (cit. on p. 115).

[234] Z. Deng, J. Pang, W. Yang, Y. Yue, B. Sharif, R. Tuli, D. Li, B. Fraass, and Z. Fan. "Four-dimensional MRI using three-dimensional radial sampling with respiratory self-gating to characterize temporal phase-resolved respiratory motion in the abdomen". Magn. Reson. Med. 75 (2016), pp. 1574-1585 (cit. on pp. 115, 117).

[235] Y. Gao, Z. Zhou, F. Han, P. J. Finn, and P. Hu. "Improved respiratory motion self-gating in cardiovascular MRI". J. Cardiov. Magn. Reson. 18 (2016), P7 (cit. on p. 115).

[236] T. Zhang, J. Y. Cheng, Y. Chen, D. G. Nishimura, J. M. Pauly, and S. S. Vasanawala. "Robust self-navigated body MRI using dense coil arrays". Magn. Reson. Med. 76 (2016), pp. 197-205 (cit. on pp. 116, 135).

[237] K. Fukunaga. Introduction to statistical pattern recognition. Elsevier, 2013 (cit. on p. 116).

[238] N. Golyandina, V. Nekrutkin, and A. A. Zhigljavsky. Analysis of time series structure: SSA and related techniques. Chapman and Hall/CRC, 2001 (cit. on p. 116).

[239] R. Vautard and M. Ghil. "Singular spectrum analysis in nonlinear dynamics, with applications to paleoclimatic time series". Physica D 35 (1989), pp. 395424 (cit. on pp. 116, 120).

[240] R. Vautard, P. Yiou, and M. Ghil. "Singular-spectrum analysis: A toolkit for short, noisy chaotic signals". Physica D 58 (1992), pp. 95-126 (cit. on pp. 116, 119, 120).

[241] D. S. Broomhead and G. P. King. "On the qualitative analysis of experimental dynamical systems". In: Nonlinear Phenomena and Chaos. Ed. by S. Sarkar. Bristol, England: Adam Hilger, 1986, pp. 113-144 (cit. on p. 116).

[242] A. Hannachi, I. T. Jolliffe, and D. B. Stephenson. "Empirical orthogonal functions and related techniques in atmospheric science: A review". Int. J. Climatol. 27 (2007), pp. 1119-1152 (cit. on p. 116).

[243] K. Koelle, X. Rodó, M. Pascual, M. Yunus, and G. Mostafa. "Refractory periods and climate forcing in cholera dynamics". Nature 436 (2005), p. 696 (cit. on p. 116).

[244] I. A. Rezek and S. J. Roberts. "Stochastic complexity measures for physiological signal analysis". IEEE Trans. Biomed. Eng. 45 (1998), pp. 1186-1191 (cit. on p. 116). 
[245] U. Kumar and V. Jain. "Time series models (Grey-Markov, Grey Model with rolling mechanism and singular spectrum analysis) to forecast energy consumption in India". Energy 35 (2010), pp. 1709-1716 (cit. on p. 116).

[246] C. L. Wu, K. Chau, and Y. Li. "Methods to improve neural network performance in daily flows prediction". J. Hydrol. 372 (2009), pp. 8o-93 (cit. on p. 116).

[247] H. Janssens, S. Hou, J. Jaeger, A.-R. Kim, E. Myasnikova, D. Sharp, and J. Reinitz. "Quantitative and predictive model of transcriptional control of the Drosophila melanogaster even skipped gene". Nat. Genet. 38 (2006), p. 1159 (cit. on p. 116).

[248] L. Feng, L. Axel, L. A. Latson, J. Xu, D. K. Sodickson, and R. Otazo. "Compressed sensing with synchronized cardio-respiratory sparsity for free-breathing cine MRI: initial comparative study on patients with arrhythmias". J. Cardiov. Magn. Reson. 16 (2014), O17 (cit. on p. 116).

[249] N. A. Campbell and W. R. Atchley. "The Geometry of Canonical Variate Analysis". Syst. Biol. 30 (1981), pp. 268-28o (cit. on p. 118).

[250] T. Harris and H. Yuan. "Filtering and frequency interpretations of singular spectrum analysis". Physica D 239 (2010), pp. 1958-1967 (cit. on pp. 119, 120).

[251] K. Kume. "Interpretation of singular spectrum analysis as complete eigenfilter decomposition". Adv. Adapt. Data Anal. 4 (2012), p. 1250023 (cit. on p. 119).

[252] M. C. Leles, A. S. Cardoso, M. G. Moreira, H. N. Guimarães, C. M. Silva, and A. Pitsillides. "Frequency-domain characterization of singular spectrum analysis eigenvectors". In: IEEE Intern. Symp. Signal Process. and Info. Tech. (ISSPIT). Vol. 16. Limassol, 2016, pp. 22-27 (cit. on p. 119).

[253] S. Xu, H. Hu, L. Ji, and P. Wang. "Embedding dimension selection for adaptive singular spectrum analysis of EEG signal". Sensors 18 (2018), p. 697 (cit. on p. 119).

[254] G. Plaut and R. Vautard. "Spells of low-frequency oscillations and weather regimes in the Northern Hemisphere". J. Atmos. Sci. $5^{1}$ (1994), pp. 210-236 (cit. on p. 120).

[255] N. Golyandina and A. Shlemov. "Variations of singular spectrum analysis for separability improvement: non-orthogonal decompositions of time series". Stat. Interface 8(3) (2015), pp. 277-294 (cit. on pp. 120, 137).

[256] A. Groth and M. Ghil. "Multivariate singular spectrum analysis and the road to phase synchronization". Phys. Rev. E 84 (2011), p. 036206 (cit. on p. 120). 
[257] M. Ghil et al. "Advanced spectral methods for climatic time series". Rev. Geophys. 40 (2002), pp. 3-1-3-41 (cit. on p. 120).

[258] J. Liu, P. Spincemaille, N. C. F. Codella, T. D. Nguyen, M. R. Prince, and Y. Wang. "Respiratory and cardiac self-gated free-breathing cardiac CINE imaging with multiecho 3D hybrid radial SSFP acquisition". Magn. Reson. Med. 63 (2010), pp. 1230-1237 (cit. on pp. 121, 135).

[259] K. Nehrke, P. Bornert, D. Manke, and J. C. Bock. "Free-breathing cardiac MR imaging: study of implications of respiratory motion-initial results". Radiology 220 (2001), pp. 810-815 (cit. on p. 121).

[26o] P. Dasari, K. Johnson, J. Dey, C. Lindsay, M. S. Shazeeb, J. M. Mukherjee, S. Zheng, and M. A. King. "MRI investigation of the linkage between respiratory motion of the heart and markers on patient's abdomen and chest: Implications for respiratory amplitude binning list-mode PET and SPECT studies". IEEE Trans. Nucl. Sci. 61 (2014), pp. 192-201 (cit. on p. 121).

[261] P. K. Dasari, A. Könik, P. H. Pretorius, K. L. Johnson, W. P. Segars, M. S. Shazeeb, and M. A. King. "Correction of hysteretic respiratory motion in SPECT myocardial perfusion imaging: Simulation and patient studies". Med. Phys. 44 (2017), pp. 437-450 (cit. on p. 121).

[262] B. Berman, Z. Li, M. Altbach, J. Galons, D. Martin, B. Dong, P. Sharma, B. Kalb, and A. Bilgin. "How to Stack the Stars: A Variable Center-Dense KSpace Trajectory for 3D MRI". In: Proc. Intl. Soc. Mag. Reson. Med. Vol. 21. Salt Lake City, 2013, p. 3829 (cit. on p. 123).

[263] J. Y. Cheng, T. Zhang, M. T. Alley, M. Uecker, M. Lustig, J. M. Pauly, and S. S. Vasanawala. "Comprehensive Multi-Dimensional MRI for the Simultaneous Assessment of Cardiopulmonary Anatomy and Physiology". Sci. Rep. 7 (2017), p. 5330 (cit. on p. 124).

[264] H. C. M. Holme, F. Ong, S. Rosenzweig, R. N. Wilke, M. Lustig, and M. Uecker. "ENLIVE: A Non-Linear Calibrationless Method for Parallel Imaging using a Low-Rank Constraint". In: Proc. Intl. Soc. Mag. Reson. Med. Vol. 25. Honolulu, 2017, p. 5160 (cit. on pp. 124, 192).

[265] F. Huang, S. Vijayakumar, Y. Li, S. Hertel, and G. R. Duensing. "A software channel compression technique for faster reconstruction with many channels". Magn. Reson. Imaging 26 (2008), pp. 133-141 (cit. on p. 124).

[266] M. Buehrer, K. P. Pruessmann, P. Boesiger, and S. Kozerke. "Array compression for MRI with large coil arrays". Magn. Reson. Med. 57 (2007), pp. 11311139 (cit. on p. 124).

[267] Y. Wang, S. J. Riederer, and R. L. Ehman. "Respiratory motion of the heart: kinematics and the implications for the spatial resolution in coronary imaging". Magn. Reson. Med. 33 (1995), pp. 713-719 (cit. on p. 128). 
[268] O. Bieri, M. Markl, and K. Scheffler. "Analysis and compensation of eddy currents in balanced SSFP". Magn. Reson. Med. 54 (2005), pp. 129-137 (cit. on p. 134).

[269] J. H. Duyn, Y. Yang, J. A. Frank, and J. W. van der Veen. "Simple Correction Method fork-Space Trajectory Deviations in MRI". J. Magn. Reson. 132 (1998), pp. 150-153 (cit. on p. 134).

[270] N. Golyandina and A. Zhigljavsky. Singular Spectrum Analysis for time series. Springer Science \& Business Media, 2013 (cit. on p. 136).

[271] A. Korobeynikov. "Computation- and space-efficient implementation of SSA". Stat. Interface 3 (2010), pp. 357-368 (cit. on p. 136).

[272] M. Leles, J. Sansão, L. Mozelli, and H. Guimarães. "A new algorithm in singular spectrum analysis framework: The Overlap-SSA (ov-SSA)". SoftwareX 8 (2018), pp. 26-32 (cit. on p. 136).

[273] D. Giannakis and A. J. Majda. "Nonlinear Laplacian spectral analysis for time series with intermittency and low-frequency variability". PNAS 109 (2012), pp. 2222-2227 (cit. on pp. 137, 156).

[274] J. Harmouche, D. Fourer, F. Auger, P. Borgnat, and P. Flandrin. "The sliding singular spectrum analysis: a data-driven nonstationary signal decomposition tool". IEEE Trans. Signal Process. 66 (2018), pp. 251-263 (cit. on p. 137).

[275] A. T. Hess, E. M. Tunnicliffe, C. T. Rodgers, and M. D. Robson. “Diaphragm position can be accurately estimated from the scattering of a parallel transmit RF coil at 7 T". Magn. Reson. Med. 79 (2018), pp. 2164-2169 (cit. on p. 146).

[276] F. Odille, P.-A. Vuissoz, P.-Y. Marie, and J. Felblinger. "Generalized reconstruction by inversion of coupled systems (GRICS) applied to free-breathing MRI". Magn. Reson. Med. 60 (2008), pp. 146-157 (cit. on p. 146).

[277] M. Henningsson, T. Hussain, M. S. Vieira, G. F. Greil, J. Smink, G. v. Ensbergen, G. Beck, and R. M. Botnar. "Whole-heart coronary MR angiography using image-based navigation for the detection of coronary anomalies in adult patients with congenital heart disease". J. Magn. Reson. Imaging 43 (2016), pp. 947-955 (cit. on p. 146).

[278] C. Santelli, R. Nezafat, B. Goddu, W. J. Manning, J. Smink, S. Kozerke, and D. C. Peters. "Respiratory bellows revisited for motion compensation: preliminary experience for cardiovascular MR". Magn. Reson. Med. 65 (2011), pp. 1097-1102 (cit. on p. 146). 
[279] M. Filipovic, P.-A. Vuissoz, A. Codreanu, M. Claudon, and J. Felblinger. "Motion compensated generalized reconstruction for free-breathing dynamic contrast-enhanced MRI". Magn. Reson. Med. 65 (2011), pp. 812-822 (cit. on p. 146).

[280] J. M. Kollmeier, Z. Tan, A. A. Joseph, O. Kalentev, D. Voit, K. D. Merboldt, and J. Frahm. "Real-time multi-directional flow MRI using model-based reconstructions of undersampled radial FLASH-A feasibility study". NMR Biomed. 32 (2019), e4184 (cit. on p. 153).

[281] V. Roeloffs, M. Bush, S. Anand, and M. Lustig. "Measuring and Correcting Gradient Delays in Multi-Echo Rosette Trajectories with RING". In: Proc. Intl. Soc. Mag. Reson. Med. Vol. 28. Sidney, 2020, accepted for publication (cit. on p. 153).

[282] X. Wang, S. Rosenzweig, N. Scholand, H. C. M. Holme, and M. Uecker. "Model-based Reconstruction for Simultaneous Multi-slice T1 Mapping using Single-shot Inversion-recovery Radial FLASH". arXiv (2019), p. 1909.10633 (cit. on p. 154).

[283] X. Wang, S. Rosenzweig, N. Scholand, H. Holme, and M. Uecker. "Modelbased reconstruction for simultaneous multi-slice T1 mapping using singleshot inversion-recovery radial FLASH". In: Proc. Intl. Soc. Mag. Reson. Med. Vol. 27. Montreal, 2019, p. 00314 (cit. on pp. 154, 191).

[284] S. Schaetz and M. Uecker. "A multi-GPU programming library for real-time applications". In: Int. Conf. Alg. Arch. Parallel Process. Vol. 12. Fukuoka, 2012, pp. 114-128 (cit. on p. 154).

[285] M. Steinmetz, T. Stümpfig, M. Seehase, J. Kowallick, A. Schuster, M. Uecker, J. Lotz, and T. Paul. "Impaired Exercise Tolerance after Repair of Tetralogy of Fallot-Insights from Real-Time Cardiovascular Magnetic Resonance Imaging". Thorac. Cardiov. Surg. 67 (2019), DGPK-V53 (cit. on p. 154).

[286] M. S. Nazir, R. Neji, P. Speier, F. Reid, D. Stäb, M. Schmidt, C. Forman, R. Razavi, S. Plein, T. F. Ismail, et al. "Simultaneous multi slice (SMS) balanced steady state free precession first-pass myocardial perfusion cardiovascular magnetic resonance with iterative reconstruction at 1.5 T". J. Cardiov. Magn. Reson. 20 (2018), pp. 1-11 (cit. on p. 154).

[287] Y. Yang, C. H. Meyer, F. H. Epstein, C. M. Kramer, and M. Salerno. “Wholeheart spiral simultaneous multi-slice first-pass myocardial perfusion imaging". Magn. Reson. Med. 81 (2019), pp. 852-862 (cit. on p. 154).

[288] D. Rigie, T. Vahle, T. Zhao, B. Czekella, L. J. Frohwein, K. Schäfers, and F. E. Boada. "Cardiorespiratory motion-tracking via self-refocused rosette navigators". Magn. Reson. Med. 81 (2019), pp. 2947-2958 (cit. on p. 155). 
[289] J. P. Haldar. "Low-Rank Modeling of Local k-Space Neighborhoods (LORAKS) for Constrained MRI". IEEE Trans. Med. Imag. 33 (2014), pp. 668-681 (cit. on p. 155).

[290] M. Jacob, M. P. Mani, and J. C. Ye. "Structured Low-Rank Algorithms: Theory, Magnetic Resonance Applications, and Links to Machine Learning". IEEE Signal Process. Mag. 37 (2020), pp. 54-68 (cit. on p. 155).

[291] L. Feng, S. Coppo, D. Piccini, J. Yerly, R. P. Lim, P. G. Masci, M. Stuber, D. K. Sodickson, and R. Otazo. " $5 \mathrm{D}$ whole-heart sparse MRI". Magn. Reson. Med. 79 (2018), pp. 826-838 (cit. on p. 155).

[292] T. Küstner, A. Bustin, O. Jaubert, R. Neji, C. Prieto, and R. Botnar. " 3 D Cartesian fast interrupted steady-state (FISS) imaging". Magn. Reson. Med. 82 (2019), pp. 1617-1630 (cit. on p. 155).

[293] J. A. Bastiaansen, D. Piccini, L. Di Sopra, C. W. Roy, J. Heerfordt, R. R. Edelman, I. Koktzoglou, J. Yerly, and M. Stuber. "Natively fat-suppressed ${ }_{5} \mathrm{D}$ whole-heart MRI with a radial free-running fast-interrupted steady-state (FISS) sequence at $1.5 \mathrm{~T}$ and $3 \mathrm{~T}^{\prime \prime}$. Magn. Reson. Med. 83 (2020), pp. 45-55 (cit. on p. 155).

[294] H. C. M. Holme. "Higher-Dimensional Extensions of Nonlinear Inverse Reconstruction for Magnetic Resonance Imaging". PhD thesis. Georg August Universität Göttingen, 2019 (cit. on p. 155).

[295] A. G. Christodoulou, C. Brinegar, J. P. Haldar, H. Zhang, Y.-J. L. Wu, L. M. Foley, T. K. Hitchens, Q. Ye, C. Ho, and Z.-P. Liang. “High-resolution cardiac MRI using partially separable functions and weighted spatial smoothness regularization". In: Int. Conf. IEEE Eng. Med. Bio. Vol. 32. Buenos Aires, 2010, pp. $871-874$ (cit. on p. 156).

[296] A. G. Christodoulou and S. G. Lingala. “Accelerated Dynamic Magnetic Resonance Imaging Using Learned Representations: A New Frontier in Biomedical Imaging". IEEE Signal Process. Mag. 37 (2020), pp. 83-93 (cit. on p. 156).

[297] S. Poddar, Y. Mohsin, D. Ansah, B. Thattaliyath, R. Ashwath, and M. Jacob. "Free-breathing cardiac MRI using bandlimited manifold modelling". arXiv (2018), p. 1802.08909 (cit. on p. 156).

[298] V. Roeloffs, S. Rosenzweig, H. C. M. Holme, M. Uecker, and J. Frahm. "Frequency-modulated SSFP with radial sampling and subspace reconstruction: A time-efficient alternative to phase-cycled bSFFP". Magn. Reson. Med. 81 (2019), pp. 1566-1579 (cit. on p. 190).

[299] T. Geigenfeind, S. Rosenzweig, M. Schmidt, and D. de Las Heras. "Confinement of two-dimensional rods in slit pores and square cavities". J. Chem. Phys. 142 (2015), p. 174701 (cit. on p. 190). 
[300] S. Rosenzweig, N. Scholand, H. C. M. Holme, and M. Uecker. "Robust Cardiac and Respiratory Self-Gating MRI Using Time-Delayed Embedding (SSA-FARY)". In: 3rd Inf. Conf. PBCS. Vol. 4. Göttingen, 2020, NoDo2 (cit. on p. 190).

[301] N. Scholand, X. Wang, S. Rosenzweig, and M. Uecker. "Fast Quantitiative MRI using a Generalized Bloch Model-based Reconstruction". In: 3rd Inf. Conf. PBCS. Vol. 4. Göttingen, 2020, NoDo1 (cit. on p. 190).

[302] N. Scholand, X. Wang, S. Rosenzweig, H. C. M. Holme, and M. Uecker. "Generic Quantitative MRI using Model-Based Reconstruction with the Bloch Equations". In: Proc. Intl. Soc. Mag. Reson. Med. Vol. 28. Sidney, 2020, accepted for publication (cit. on p. 190).

[303] X. Wang, S. Rosenzweig, and M. Uecker. "Model-based reconstruction for simultaneous multi-slice myocardial Ti mapping using single-shot inversionrecovery radial FLASH". In: Proc. Intl. Soc. Mag. Reson. Med. Vol. 28. Sidney, 2020, accepted for publication (cit. on p. 190).

[304] N. Scholand, S. Rosenzweig, F. Ertingshausen, and M. Uecker. "Plastic Bricks in MRI: An Efficient Way to Build Static and Dynamic Phantoms". In: Proc. Intl. Soc. Mag. Reson. Med. Vol. 27. Montreal, 2019, p. 1593 (cit. on p. 191).

[305] N. Scholand, X. Wang, S. Rosenzweig, and M. Uecker. "Generalized modelbased reconstruction for quantitative MRI using the Bloch-Equations". In: Magn. Reson. Mater. Phy. Vol. 36. Rotterdam, 2019, 32(Suppl 1): 235 (cit. on p. 191).

[306] H. C. M. Holme, S. Rosenzweig, X. Wang, and M. Uecker. “Non-linear Inverse Compressed-Sensing Reconstruction for Self-Gated Multidimensional Cardiac MRI: XD-NLINV". In: Proc. Intl. Soc. Mag. Reson. Med. Vol. 27. Montreal, 2019, p. 4753 (cit. on p. 191).

[307] A. N. Moghaddam, E. Mohammadi, S. Rosenzweig, and M. Uecker. "Real-time radial tagging with radial FLASH: preliminary results". In: Proc. Soc. Cardiovasc. Magn. Reson. Vol. 22. Bellevue, 2019, pp. 1536-1537 (cit. on p. 191).

[308] H. C. M. Holme, N. Scholand, S. Rosenzweig, and M. Uecker. "BandingFree Reconstruction in Frequency-Modulated bSSFP using Virtual Coils with Regularized Non-Linear Inversion". In: Proc. Intl. Soc. Mag. Reson. Med. Vol. 26. Paris, 2018, p. 2870 (cit. on p. 191).

[309] H. C. M. Holme, S. Rosenzweig, and M. Uecker. "Phase-constrained Magnetic Resonance Imaging as a Nonlinear Inverse Problem with a Rank Penalty". In: Proc. SIAM Conf. Img. Sci. Bologna, 2018, p. 163 (cit. on p. 191). 
[310] S. Rosenzweig, H. C. M. Holme, R. N. Wilke, and M. Uecker. "SOMS: Simultaneous Orthogonal Multi-Slice Imaging". In: Proc. Intl. Soc. Mag. Reson. Med. Vol. 24. Honolulu, 2017, p. 3845 (cit. on p. 192).

[311] S. Rosenzweig, H. C. M. Holme, R. N. Wilke, and M. Uecker. "Real-time Simultaneous Multi-Slice MRI Using Nonlinear Inverse Reconstruction: Acquisition Strategies And In-Vivo Example". In: Proc. Intl. Soc. Mag. Reson. Med. DS. Vol. 20. Göttingen, 2017, pp. 121-122 (cit. on p. 192).

[312] S. Rosenzweig, H. C. M. Holme, R. N. Wilke, and M. Uecker. “Comparison Of Sampling Schemes In Simultaneous Multi-Slice MRI Using Nonlinear Inverse Reconstruction". In: 3rd Inf. Conf. PBCS. Vol. 3. Göttingen, 2017, P1.11 (cit. on p. 192).

[313] H. C. M. Holme, S. Rosenzweig, F. Ong, M. Lustig, and M. Uecker. “ENLIVE: An Efficient Nonlinear Method for Calibrationless and Robust Parallel Imaging". In: Proc. Intl. Soc. Mag. Reson. Med. DS. Vol. 20. Göttingen, 2017, pp. 79-80 (cit. on p. 192).

[314] H. C. M. Holme, S. Rosenzweig, F. Ong, R. N. Wilke, M. Lustig, and M. Uecker. In: 3rd Inf. Conf. PBCS. Vol. 3. Göttingen, 2017, P4.06 (cit. on p. 192). 


\title{
CURRICULUM VITAE
}

\author{
SEBASTIAN ROSENZWEIG \\ Born on 1st August 1991 in Würzburg
}

CAREER

2019 Internship

Visiting research fellow at the Boston Children's Hospital and

Harvard Medical School, Boston, Massachusetts, USA, under Prof. M. Moghari

SINCE 2016 PhD Student

with Prof. Dr. Martin Uecker (supervisor)

at the University Medical Center Göttingen

in the International Max Planck Research School (Physics of Biological and

Complex Systems)

of the Göttingen Graduate School for Neuroscience, Biophysics and Molec-

ular Biosciences (GGNB)

2014-2016 Master's degree in Physics

Examination: Grade "1.0" (very good), with distinction

with Prof. Dr. Martin Uecker (supervisor)

at the Georg-August University Göttingen, Germany

Thesis title: "Simultaneous multi-slice reconstruction by Regularized Nonlinear Inversion"

2014 Internship

at the Dr. Johannes Heidenhain GmbH, Traunreut, Germany

2013-2014 International Voluntary Service

social work for a Non-Government Organization in Argentina and Ecuador

2010-2013 Bachelor's degree in Physics

Examination: Grade "1.0" (very good), with distinction

with Prof. Dr. Matthias Schmidt (supervisor)

at the University of Bayreuth, Germany

Thesis title: "Monte Carlo simulation of two-dimensional hard rectangles confined in slab geometry"

2001-2010 Johann-Schöner-Gymansium Karlstadt, Germany

Abitur: Grade "1.0" (very good) 


\section{JOURNAL PUBLICATIONS}

2020 S. Rosenzweig, N. Scholand, H. C. M. Holme, and M. Uecker. "Cardiac and Respiratory Self-Gating in Radial MRI using an Adapted Singular Spectrum Analysis (SSA-FARY)". IEEE Trans. Med. Imag. (2020). doi: 10.1109/TMI.2020.2985994

2019 S. Rosenzweig, H. C. M. Holme, and M. Uecker. "Simple auto-calibrated gradient delay estimation from few spokes using Radial Intersections (RING)". Magn. Reson. Med. 81 (2019), pp. 1898-1906

H. C. M. Holme, S. Rosenzweig, F. Ong, R. N. Wilke, M. Lustig, and M. Uecker. "ENLIVE: An Efficient Nonlinear Method for Calibrationless and Robust Parallel Imaging". Sci. Rep. 9 (2019), p. 3034

V. Roeloffs, S. Rosenzweig, H. C. M. Holme, M. Uecker, and J. Frahm. “Frequencymodulated SSFP with radial sampling and subspace reconstruction: A time-efficient alternative to phase-cycled bSFFP". Magn. Reson. Med. 81 (2019), pp. 1566-1579

2018 GöVIP-16 Clinical Science Award

S. Rosenzweig, H. C. M. Holme, R. N. Wilke, D. Voit, J. Frahm, and M. Uecker. "Simultaneous multi-slice MRI using cartesian and radial FLASH and regularized nonlinear inversion: SMS-NLINV". Magn. Reson. Med. 79 (2018), pp. 2057-2066

2015 T. Geigenfeind, S. Rosenzweig, M. Schmidt, and D. de Las Heras. "Confinement of two-dimensional rods in slit pores and square cavities". J. Chem. Phys. 142 (2015), p. 174701. Shared First Author

\section{CONFERENCE ABSTRACTS}

2020 S. Rosenzweig, N. Scholand, H. C. M. Holme, and M. Uecker. "Robust Cardiac and Respiratory Self-Gating MRI Using Time-Delayed Embedding (SSA-FARY)". In: 3 rd Inf. Conf. PBCS. Vol. 4. Göttingen, 2020, NoDo2

N. Scholand, X. Wang, S. Rosenzweig, and M. Uecker. "Fast Quantitiative MRI using a Generalized Bloch Model-based Reconstruction". In: 3rd Inf. Conf. PBCS. Vol. 4. Göttingen, 2020, NoDo1

N. Scholand, X. Wang, S. Rosenzweig, H. C. M. Holme, and M. Uecker. “Generic Quantitative MRI using Model-Based Reconstruction with the Bloch Equations". In: Proc. Intl. Soc. Mag. Reson. Med. Vol. 28. Sidney, 2020, accepted for publication

X. Wang, S. Rosenzweig, and M. Uecker. "Model-based reconstruction for simultaneous multi-slice myocardial $\mathrm{T}_{1}$ mapping using single-shot inversion-recovery radial FLASH". In: Proc. Intl. Soc. Mag. Reson. Med. Vol. 28. Sidney, 2020, accepted for publication

2019 ISMRM Merit Award, magna cum laude

S. Rosenzweig, H. Holme, and M. Uecker. "Simple Auto-Calibrated Gradient Delay Estimation From Few Spokes Using Radial Intersections (RING) for Interactive Realtime MRI". In: Proc. Intl. Soc. Mag. Reson. Med. Vol. 27. Montreal, 2019, p. 445 
S. Rosenzweig, N. Scholand, H. Holme, and M. Uecker. "Robust Cardiac and Respiratory Self-Gating Using an Adapted Singular Spectrum Analysis (SSA-FARI): Application to Simultaneous-Multi-Slice Imaging". In: Proc. Intl. Soc. Mag. Reson. Med. Vol. 27. Montreal, 2019, p. 2444

S. Rosenzweig, N. Scholand, H. Holme, and M. Uecker. "Robust Cardiac and Respiratory Self-Gating using an adapted Singular Spectrum Analysis". In: Proc. Soc. Cardiovasc. Magn. Reson. Vol. 22. Bellevue, 2019, pp. 580-582

N. Scholand, S. Rosenzweig, F. Ertingshausen, and M. Uecker. "Plastic Bricks in MRI: An Efficient Way to Build Static and Dynamic Phantoms". In: Proc. Intl. Soc. Mag. Reson. Med. Vol. 27. Montreal, 2019, p. 1593. Shared First Author

ISMRM Merit Award, magna cum laude

X. Wang, S. Rosenzweig, N. Scholand, H. Holme, and M. Uecker. "Model-based reconstruction for simultaneous multi-slice $\mathrm{T}_{1}$ mapping using single-shot inversionrecovery radial FLASH". In: Proc. Intl. Soc. Mag. Reson. Med. Vol. 27. Montreal, 2019, p. 00314

N. Scholand, X. Wang, S. Rosenzweig, and M. Uecker. “Generalized model-based reconstruction for quantitative MRI using the Bloch-Equations". In: Magn. Reson. Mater. Phy. Vol. 36. Rotterdam, 2019, 32(Suppl 1): 235

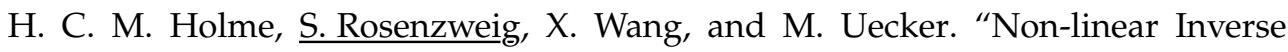
Compressed-Sensing Reconstruction for Self-Gated Multidimensional Cardiac MRI: XD-NLINV". In: Proc. Intl. Soc. Mag. Reson. Med. Vol. 27. Montreal, 2019, p. 4753

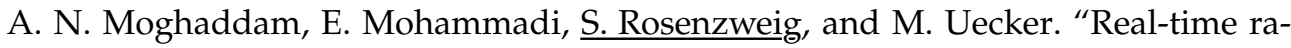
dial tagging with radial FLASH: preliminary results". In: Proc. Soc. Cardiovasc. Magn. Reson. Vol. 22. Bellevue, 2019, pp. 1536-1537

2018 ISMRM Merit Award, summa cum laude

S. Rosenzweig, H. C. M. Holme, N. Scholand, R. N. Wilke, and M. Uecker. "SelfGated and Real-time Simultaneous Multi-Slice Cardiac MRI from the Same Acquisition". In: Proc. Intl. Soc. Mag. Reson. Med. Vol. 26. Paris, 2018, p. 0209

S. Rosenzweig, H. C. M. Holme, R. N. Wilke, and M. Uecker. "Extending the Simple Method for Adaptive Gradient-Delay Compensation in Radial MRI". In: Proc. Intl. Soc. Mag. Reson. Med. Vol. 26. Paris, 2018, p. 4207

H. C. M. Holme, N. Scholand, S. Rosenzweig, and M. Uecker. "Banding-Free Reconstruction in Frequency-Modulated bSSFP using Virtual Coils with Regularized NonLinear Inversion". In: Proc. Intl. Soc. Mag. Reson. Med. Vol. 26. Paris, 2018, p. 2870

H. C. M. Holme, S. Rosenzweig, and M. Uecker. "Phase-constrained Magnetic Resonance Imaging as a Nonlinear Inverse Problem with a Rank Penalty". In: Proc. SIAM Conf. Img. Sci. Bologna, 2018, p. 163

2017 S. Rosenzweig, H. C. M. Holme, R. N. Wilke, and M. Uecker. "Simultaneous MultiSlice Real-Time Imaging with Radial Multi-Band FLASH and Nonlinear Inverse Reconstruction". In: Proc. Intl. Soc. Mag. Reson. Med. Vol. 24. Honolulu, 2017, p. 0518 
S. Rosenzweig, H. C. M. Holme, R. N. Wilke, and M. Uecker. "SOMS: Simultaneous Orthogonal Multi-Slice Imaging". In: Proc. Intl. Soc. Mag. Reson. Med. Vol. 24. Honolulu, 2017, p. 3845

S. Rosenzweig, H. C. M. Holme, R. N. Wilke, and M. Uecker. "Real-time Simultaneous Multi-Slice MRI Using Nonlinear Inverse Reconstruction: Acquisition Strategies And In-Vivo Example". In: Proc. Intl. Soc. Mag. Reson. Med. DS. Vol. 20. Göttingen, 2017, pp. 121-122

S. Rosenzweig, H. C. M. Holme, R. N. Wilke, and M. Uecker. “Comparison Of Sampling Schemes In Simultaneous Multi-Slice MRI Using Nonlinear Inverse Reconstruction". In: 3rd Inf. Conf. PBCS. Vol. 3. Göttingen, 2017, P1.11

H. C. M. Holme, F. Ong, S. Rosenzweig, R. N. Wilke, M. Lustig, and M. Uecker. "ENLIVE: A Non-Linear Calibrationless Method for Parallel Imaging using a LowRank Constraint". In: Proc. Intl. Soc. Mag. Reson. Med. Vol. 25. Honolulu, 2017, p. 5160

H. C. M. Holme, S. Rosenzweig, F. Ong, M. Lustig, and M. Uecker. "ENLIVE: An Efficient Nonlinear Method for Calibrationless and Robust Parallel Imaging". In: Proc. Intl. Soc. Mag. Reson. Med. DS. Vol. 20. Göttingen, 2017, pp. 79-80

H. C. M. Holme, S. Rosenzweig, F. Ong, R. N. Wilke, M. Lustig, and M. Uecker. In: 3rd Inf. Conf. PBCS. Vol. 3. Göttingen, 2017, $\mathrm{P} 4.06$

2016 S. Rosenzweig and M. Uecker. "Reconstruction of multiband MRI data using Regularized Nonlinear Inversion". In: Proc. Intl. Soc. Mag. Reson. Med. Vol. 24. Singapore, 2016, p. 3259

\section{LANGUAGES}

GERMAN Native language

ENGLISH Fluent (speaking, reading, writing)

SPANISH Advanced (speaking, reading, writing)

FRENCH Basic 